\title{
FOSTER PARENTS' UNDERSTANDING OF THE FOSTER CHILD'S PERSPECTIVE: DOES IT MATTER AND CAN IT BE CHANGED?
}

\author{
by \\ WENDY JOAN KELLY
}

\begin{abstract}
A thesis
submitted to Victoria University of Wellington in fulfilment of the requirements for the degree of Doctor of Philosophy
\end{abstract}

Victoria University of Wellington 



\begin{abstract}
Socio-emotional outcomes for children placed in care are more positive when foster parents have a secure state of mind regarding attachment and are able to think about the child's unique psychological perspective (Dozier, Stovall, Albus \& Bates, 2001; Schofield \& Beek, 2005a). One aspect of parents' thinking about the child has been conceptualised as mind-mindedness (Meins, 1998) and is measured by the proportion of mind-related comments made when a parent is asked to describe their child. The first study examines whether foster parent's mind-mindedness and attachment perceptions, amongst other child and foster parent characteristics, are positively associated with the foster parent-child relationship and the child's emotional and behavioural outcomes.

The study found that foster parents with higher mind-mindedness had foster children with fewer behaviour problems and this relationship was enhanced by foster parents having positive attachment perceptions. Moreover, foster parent mind-mindedness was not related to the quality of the parent-child relationship, except when parents had positive attachment perceptions. Regarding the child's placement characteristics, the number of previous placements the child had experienced predicted the child's emotional and behaviour problems and the age at which the child was placed predicted the quality of the foster parent-child relationship. The best placement predictors of both outcome variables considered together were the age at which the child was placed and short term placements.

Drawing on attachment theory, and guided by the findings of study one, the author developed a a training programme and a Relational Learning Framework (RLF) to assist foster parents and foster care practitioners to understand the child's psychological perspective. The second study employed a multiple- baseline design to evaluate the effectiveness of the RLF guided training programme. Statistically significant improvements were found regarding parents' reports of children's behaviour problems, parents' daily stress, the attachment relationship and children's overall functioning at post-test. However, at follow-up, only the children's overall functioning remained significantly improved, although increases in positive mind-mindedness became statistically significant, relative to post-test. Some foster parents showed decreases in wellbeing scores, relative to their scores at pre- and post-test.

The third study used a pre- post-test design to evaluate the effectiveness of the training programme to assist foster care practitioners to apply the RLF in their clinical practice and to
\end{abstract}


deliver the training programme to foster parents. At post-test, practitioners' empathy and reflectiveness showed a statistically significant increase but no statistically significant increases were found in practitioners' mind-mindedness. The training was rated highly by participants and a thematic analysis of diary entries showed that they used the RLF in their practice, were able to help foster parents understand the child's perspective, and reported positive therapeutic gains from utilising these techniques.

The research provides preliminary evidence that foster parent mind-mindedness, in conjunction with attachment perceptions, is associated with the child's emotional and behaviour problems and the foster parent-child relationship. The results from the intervention study with foster parents showed initially promising gains, which were not maintained at follow-up, and the results from the foster care practitioners study indicated improvements in practitioners' empathy and reflectiveness, as well as positive outcomes of using the training material. 


\section{Acknowledgements}

I would like to thank my primary supervisor, Associate Professor Karen Salmon, who guided the development of this thesis with good humour and sound advice, my second supervisor, Professor Tony Ward for clear and incisive input when needed and my third supervisor, Professor Neville Blampied from Canterbury University, who helped me navigate the world of single case research. Thanks also to Associate Professor Paul Jose, who helped me with mediation, moderation and path analysis, and whose book was invaluable.

I am grateful for the support of my friends and family. Thanks are especially due to Lesley Ayland for her emotional and practical support, and grounding discussions.. Thanks to Finn, Felix and Jamie for reminding me there is a wider world out there.

I would also like to thank all the agencies who took part in this research or facilitated access to participants, especially Child, Youth and Family, Barnardos, Open Homes, Fostering Kids, and the agencies who took part in my two intervention studies. Personal thanks are due to Nova Salomen, Pam Pilalis, Andeana Pilalis, the Ministry of Social Development Research Access Committee, Suzanne Stewart, Andrea McKenzie, Sarah Packman, and Lynne White. And last but not least, thank you to all the foster parents and foster care practitioners who took part in my research.

Thanks are due to my hard working Research Asssistants for the project: Dora (Dee) SharpDavidson, Sarah Moshenrose, Zara Mansoor and Tania Stanton, and to Giselle Bahr for her competent coding of the EA videos. Thank you to my colleagues in the clinical programme, who encouraged me, put up with my recent distraction, and made my work load lighter. Special thanks to Deirdre Brown, Clare-Ann Fortune, Ben Sedley and Martin Boswell for your support and technical assistance. I would also like to thank the School of Psychology, the Todd Foundation and the NZ Federation of Graduate Women for their financial support, Marc Wilson for allowing me six weeks of productive sabbatical and the Faculty of Science for a conference grant.

Thank you to the thesis whisperer, Lizzie Towl, for her support, organising Shut Up and Write groups and $\mathrm{PhD}$ Bootcamp, which enabled the finishing of the thesis. Lastly, thanks to the VUW Bootcampers for making the Saturday National Library study group supportive and entertaining. 


\section{Table of Contents}

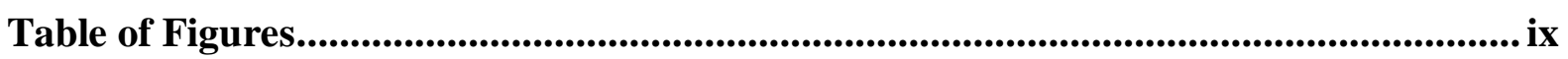

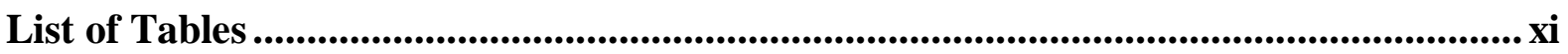

Chapter One - Introduction: Foster care and child maltreatment ........................................ 1

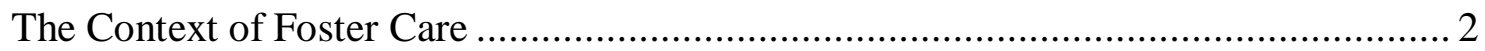

Child Maltreatment in New Zealand................................................................. 6

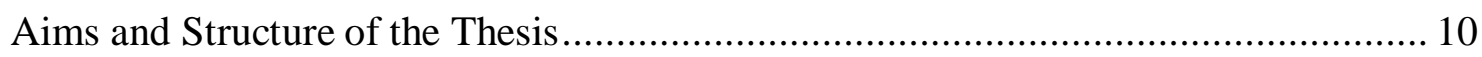

Chapter Two-Foster care: Child mental health and outcomes......................................... 13

Prevalence of Mental Health Problems: Studies including both Foster Children and

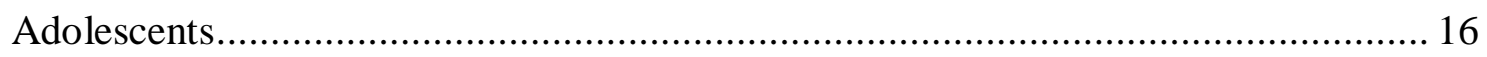

Correlates and predictors of mental health problems............................................... 18

Prevalence of mental health problems in samples of adolescents only ......................... 21

Prevalence of mental health problems in adults formerly in foster care....................... 22

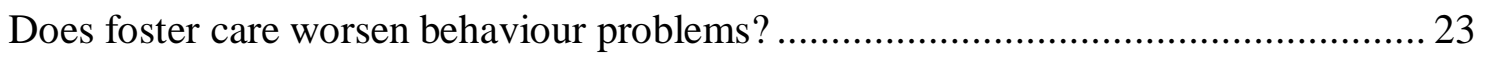

Factors associated with placement disruption ....................................................... 25

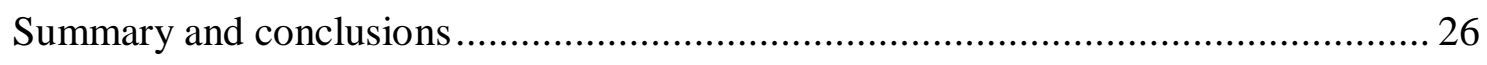

Chapter Three-Psychological factors in foster care: Attachment, mind-mindedness

and emotional availability ................................................................................................... 29

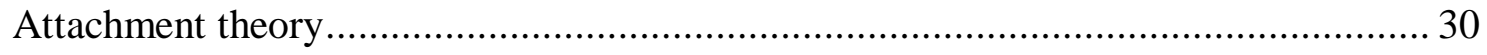

Attachment research in foster care...................................................................... 31

Mental Representations and Mentalisation ........................................................... 34

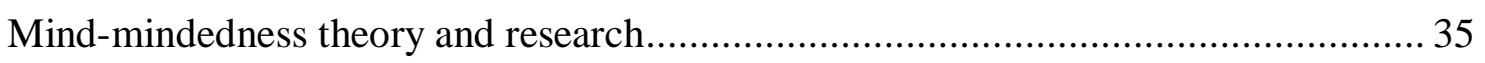

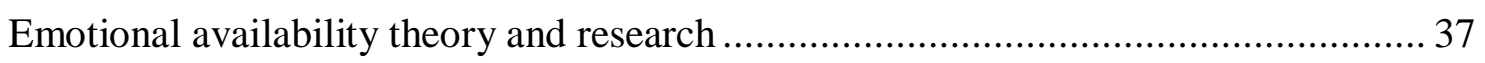

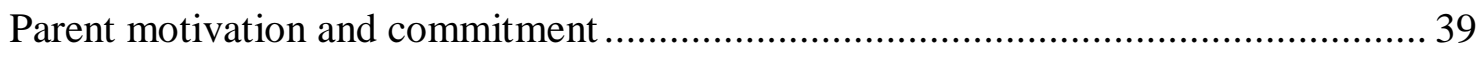

Chapter Four-Study 1: The relationship between foster child characteristics, foster

parent psychological functioning, and two child outcomes ......................................... 41

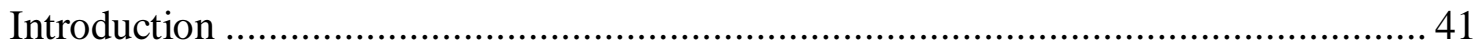

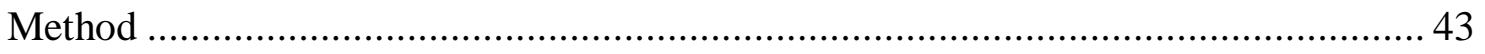

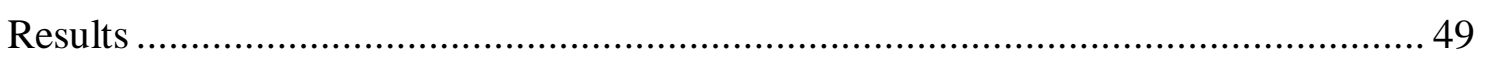

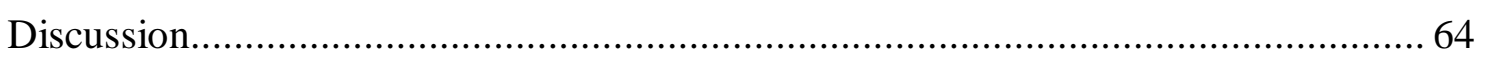

Foster parent mind-mindedness and the quality of the parent-child relationship.......... 67 
Chapter Five-Training programmes and therapeutic intervention in foster care ....... 77

Limitations and Issues in Foster Care Research .................................................... 78

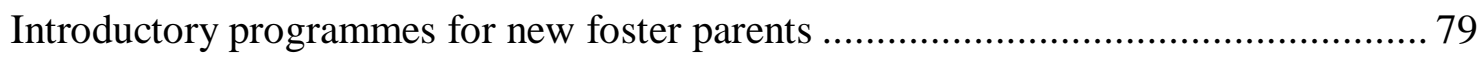

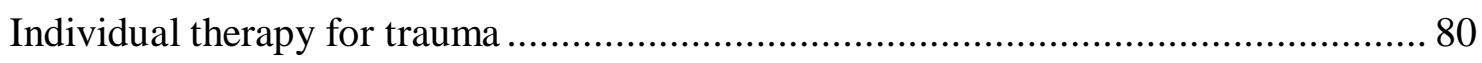

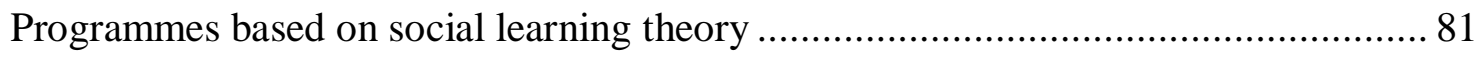

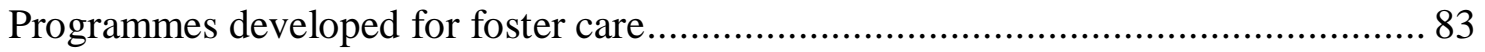

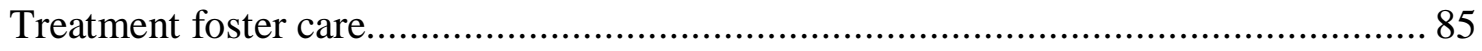

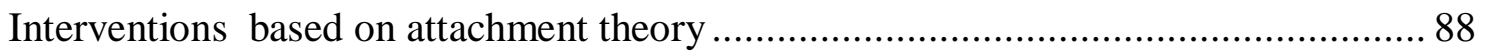

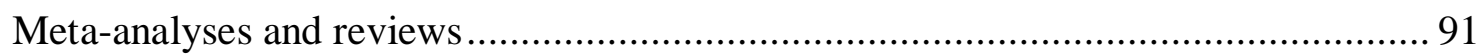

Chapter Six-Helping foster parents understand the foster child's perspective: A relational learning framework for foster care ......................................................... 95

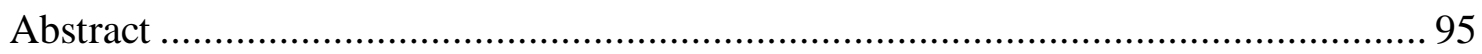

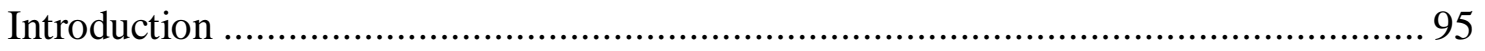

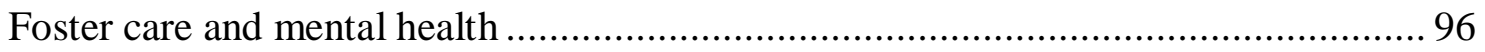

Treatment for children in foster care ............................................................... 98

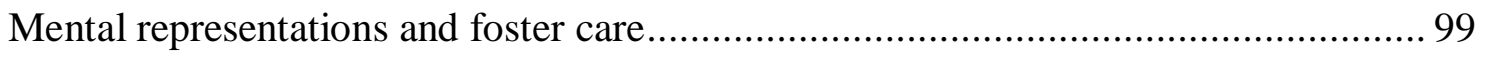

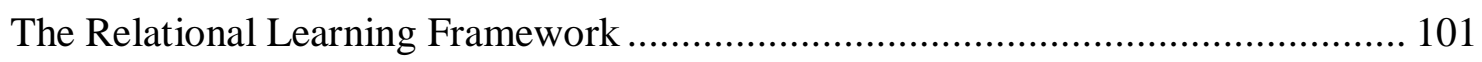

Applying the Relational Learning Framework ................................................ 102

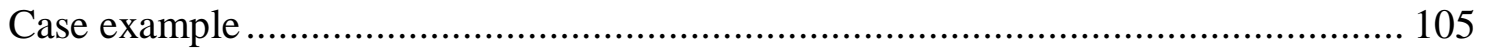

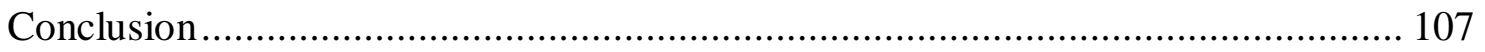

Chapter Seven-Study 2: A multiple-baseline study of the effectiveness of a training programme for foster parents .................................................................. 109

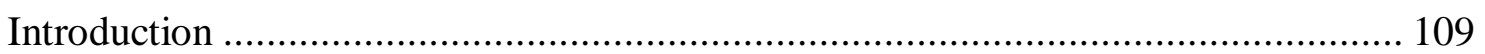

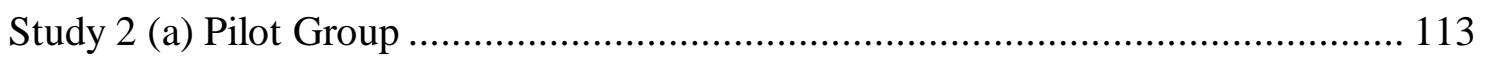

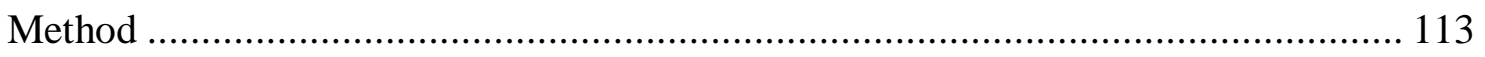

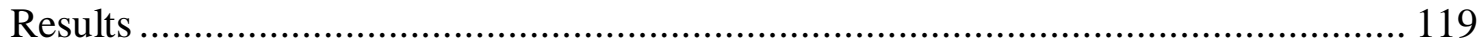

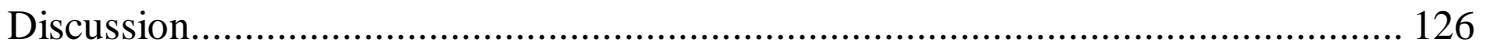

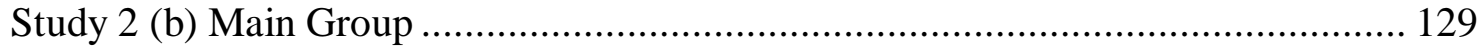

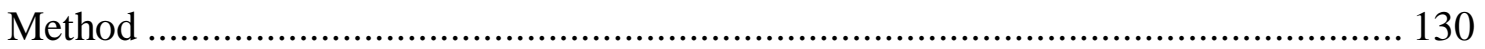

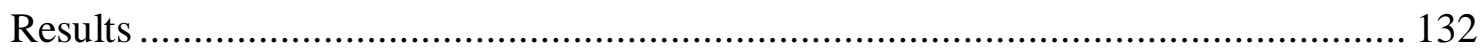

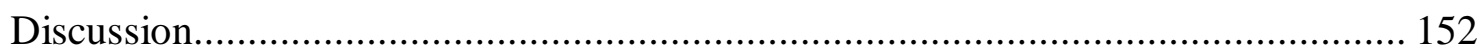


Chapter Eight -Study 3: An evaluation of a training programme in foster care for

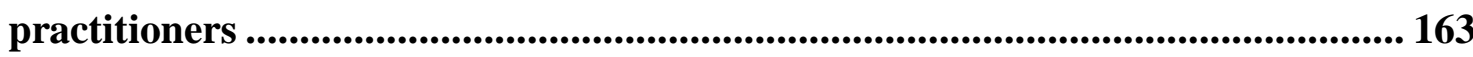

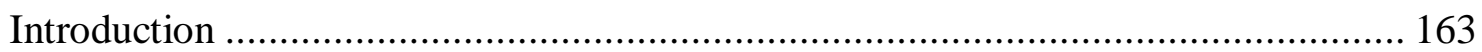

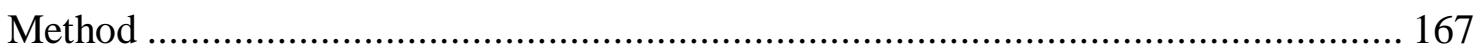

The Lightbulb training programme for practitioners ............................................. 171

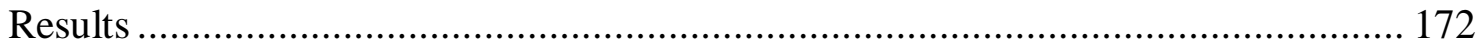

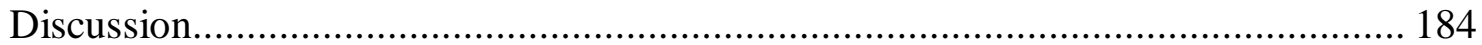

Chapter Nine—Overall discussion and conclusions ................................................ 191

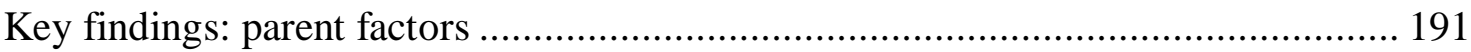

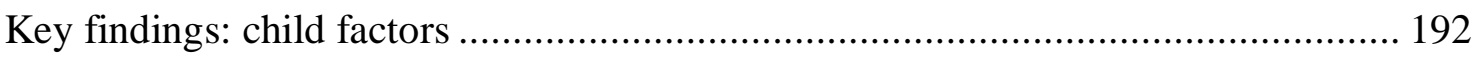

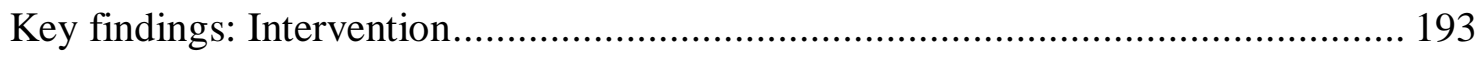

Implications for practice and future directions ................................................. 195

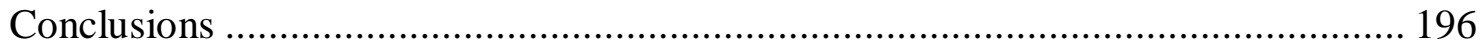

Appendix A—Group Study Interview (semi-structured) ............................................ 199

Appendix B-Correlations for the Emotional Availability Scale $\left(4^{\text {th }}\right.$ ed.) $\ldots . . . . . . . . . . . . . . .202$

Appendix C-Articulated thoughts in simulated situations (ATSS) procedure ........... 203

Appendix D_ATSS coding ....................................................................................... 210

Appendix E_Practitioner Interview (semi-structured) .............................................. 213

Appendix F_Coding Scheme: Study 3: Practitioner Diaries ........................................ 214

References................................................................................................................................... 217 


\section{Table of Figures}

Figure 1. Moderation of relationship between mind-mindedness and the child's behaviour problems, by parent attachment perceptions.

Figure 2. Moderation of relationship between parent mind-mindedness and the foster parent-child relationship, by parent

Figure 3. Moderation of relationship between parent mind-mindedness and the foster parent-child relationship, by parent

Figure 4. Moderation of relationship between parent attachment perceptions and the quality of the foster parent-child relationship, by child's gender.

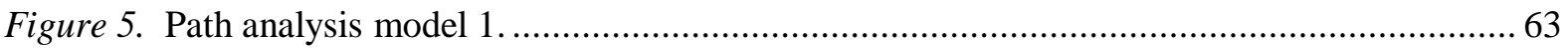

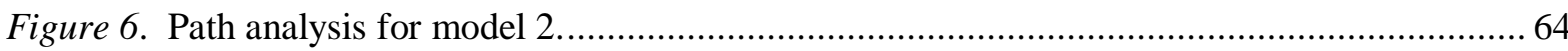

Figure 7. Modified Brinley Plot showing line of no change and pre- and post cut off areas for graph interpretation.

Figure 8. Modified Brinley plots showing pre-intervention scores on the $\mathrm{X}$ axis and post-intervention or follow-up scores on the $\mathrm{Y}$ axis for each measure and sub-scale.

Figure 9. Level of Parent Daily Report: Child Behaviour for participants $1-4$.

Figure 10. Modified Brinley plots showing pre-intervention scores for mind-mindedness, positive mind-mindedness and negative mind-mindedness on the $\mathrm{X}$ axis and post-intervention or follow-up scores on the Y axis.

Figure 11. Modified Brinley plots showing time 1 pre-intervention scores for the Behavioural Incident Interview on the $\mathrm{X}$ axis and post-intervention and follow-up scores on the $\mathrm{Y}$ axis.

Figure 12. Modified Brinley plots showing pre-intervention scores for Attributions on the $\mathrm{X}$ axis and post-intervention and follow-up scores on the $\mathrm{Y}$ axis.

Figure 13. Modified Brinley plots showing pre-intervention scores for the Strengths and Difficulties

Questionnaire on the $\mathrm{X}$ axis and post-intervention and follow-up scores on the $\mathrm{Y}$ axis. 138

Figure 14. Modified Brinley plots showing pre-intervention scores for the Parent Daily Report: Child behaviour on the $\mathrm{X}$ axis and post-intervention and follow-up scores on the $\mathrm{Y}$ axis.

Figure 15. Modified Brinley plots showing pre-intervention scores for the Parent Daily Report: Parent Stress on the $\mathrm{X}$ axis and post-intervention and follow-up scores on the $\mathrm{Y}$ axis.............................. 140

Figure 16. Modified Brinley plots showing pre-intervention scores for the Outcome Rating Scale on the $\mathrm{X}$ axis and post-intervention and follow-up scores on the $\mathrm{Y}$ axis.

Figure 17. Modified Brinley plots showing time 1 pre-intervention scores for the Caregiver Strain Questionnaire on the $\mathrm{X}$ axis and time 2 and time 3 scores on the $\mathrm{Y}$ axis.

Figure 18. Modified Brinley plots showing pre-intervention scores for the Quality of Attachment Relationship Questionnaire on the $\mathrm{X}$ axis and post-intervention and follow-up scores on the $\mathrm{Y}$ axis.

Figure 19. Modified Brinley plots showing pre-intervention scores for the Depression, Anxiety and Stress Scale on the $\mathrm{X}$ axis and time 2 and time 3 scores on the $\mathrm{Y}$ axis.

Figure 20. Modified Brinley plots comparing week 1, 2, 3 and 4 of Parent Daily Report: Child Behaviour scores.

Figure 21. Multiple-baseline across participants graphs for each participant for baseline, treatment phase and follow-up for the Parent Daily Report: Child behaviour.

Figure 22. Multiple-baseline across participants time-series data for Outcome Rating Scale. 
Figure 23. Modified Brinley plots showing pre-intervention scores for the mind-mindedness, positive mind-mindedness and negative mind-mindedness on the $\mathrm{X}$ axis and post-intervention scores on the $\mathrm{Y}$ axis.

Figure 24. Modified Brinley plots showing pre-intervention scores for the Articulated Thoughts in Simulated Situations paradigm on the $\mathrm{X}$ axis and post-intervention scores on the $\mathrm{Y}$ axis. 


\section{List of Tables}

Table 1 Foster Parents' and Children's Demographic and Placement Variables.

Table 2 Means, Standard Deviations, Range and Clinical Cut-offs for Measures, where available ... 50

Table 3 Correlations amongst measures

Table 4 Hierarchical Regression Model Predicting Quality of the Foster Parent-child Relationship and Foster Child Emotional and Behavioural Problems .....

Table 5 Hierarchical Regression Model Predicting Quality of the Foster Parent-child Relationship and Foster Child Emotional and Behavioural Problems using an Interaction Term..

Table 6 Hierarchical regression model predicting quality of the foster parent-child relationship and foster child emotional and behavioural problems using an interaction term.....

Table 7 Regression weights for path analysis model 1

Table 8 Regression weights for path analysis model 2.

Table 9 The Relational Learning Framework. 108

Table 10 Demographic Information of Participants ..... 116

Table 11 Means, (Standard Deviations) at Pre- and Post-intervention, Effect sizes (Cohen's d) and Probability values for $t$ (2 tailed) comparing Pre and Post-intervention Means.

Table 12 Percentage of Treatment Scores Exceeding the Median of the Baseline for the First and Second Halves of the Treatment Phase and Overall....

Table 13 Demographic Information of Participants in Group 1and 2.

Table 14 Demographic Information of Participants in Group 3 (Same Agency as Pilot Group).....

Table 15 Demographic Information of Participants in Group 4

Table 16 Mean, Standard Deviations, $t$-tests and Effect sizes for Mind-mindedness and the

Behavioural Incident Interview at Pre- and Post-interventionfor all groups (1-4) .....

Table 17 Mean, Standard Deviations, t-tests and Effect sizes for Mind-mindedness and the

Behavioural Incident Interview for the Follow-up Group at Pre-intervention and Follow-up ......... 133

Table 18 Means, Standard Deviations and t-tests from Pre-to Post-intervention $(N=15)$......

Table 19 Means, Standard Deviations and t-tests for Pre-intervention (Follow-up Group Only) to Follow-up $(N=9)$....

Table 20 Number of Participants Showing Clinically Significant Change on the Outcome Rating Scale at Pre-intervention, Post-intervention and Follow-up 141

Table 21 Length of baseline for each group

Table 22 Percentage of Treatment Scores Exceeding the Median of the Baseline for the First and Second Halves of the Treatment Phase and Overall....

Table 23 Worked example of the "Iceberg exercise" used for problem solving....

Table 24 Means and Standard Deviations for the Articulated Thoughts in Simulated Situations Interview and Mind-mindedness

Table 25 Means and Standard Deviations for the Training Evaluation Form at post-test and 3 month follow-up).

Table 26 Means and Standard Deviations for the Human Services Training Effectiveness Postcard (HSTEP) at 3 month follow-up.... 175

Table 27 Main themes of thematic analysis. 176 


\section{Chapter One-Introduction: Foster care and child maltreatment}

\section{Statement of the Problem}

Children who come into foster care constitute one of the most vulnerable groups in society and typically experience maltreatment, parent substance abuse or mental health issues, poverty, and separation from their families. Rejection and distrust of new caregivers is common, even in very young children (Stovall-McClough \& Dozier, 2004). Foster children have been found to have two to three times the rate of developmental, emotional and behavioural problems of other children (Burns et al., 2004, Keil \& Price, 2006). Despite these well documented problems, many foster parents do not receive the training required to assist the child and foster children often do not have access to mental health services (Burns et al., 2004; Stahmer et al., 2005). The primary function of foster care is seen as the provision of a stable and safe environment for children, with the role of assisting a child with any difficulties belonging outside of the foster family, with health and education providers (Horwitz, Chamberlain, Landsverk, \& Mullican, 2010).

Studies have shown that children's behaviour does not improve through foster care alone, and may actually deteriorate while in care (Biehal, Ellison, Baker, \& Sinclair, 2011; Lawrence, Carlson \& Egeland, 2006; Wilson 2006). If intervention programmes are not put in place to address foster children's social and psychological difficulties, these problems can become compounded, with adolescents and adults who have left foster care often experiencing social and economic disadvantage such as teen pregnancy, unemployment, and homelessness. Although effective interventions have been developed to improve the mental health problems of foster children, these programmes are particularly targeted at preschoolers and adolescents, and are not widely available (Leve et al., 2012). Innovative, affordable interventions across a broad age range are required.

Given these constraints in foster care, it is important to understand the barriers facing foster parents in managing foster children's high levels of behaviour problems. An important barrier is likely to be the considerable cognitive demands on foster parents to interpret and manage a child's oppositional behaviour, which is typically complex in presentation. Foster children's challenging behaviour can compromise foster parents' ability to form nurturing relationships with them and to help children learn more adaptive self-management skills (Bernier, Ackerman, \& Stovall-McClough, 2004). An important gap in the research literature is the investigation of the role of the foster parents' own psychological functioning in this skill acquisition process. Most foster care research investigates the prevalence of 
mental health problems in foster children and seeks to identify the causes of placement disuption. A small number of studies have examined foster parents' characteristics such as commitment to the child, motivation for providing foster care, expectations of length of placement, parenting skills and general family functioning. In order to address the gap in the foster care literature, this thesis focuses on the concept of mind-mindedness (Meins, 1997). Mind-mindedness refers to parents' ability to treat the child as a separate individual in their own right and with their own mental life including thoughts, emotions and wishes (Meins, 1998; Meins \& Fernyhough, 2010). The current research into foster parent mind-mindedness is carried out by way of a descriptive study and two intervention studies.

Chapter One focuses on the the incidence and context of foster care, the incidence of child maltreatment, and selected effects on children's development. The aims and structure of the thesis are then outlined.

\section{Terms Used in this Thesis}

Throughout this thesis, I have adopted the term "foster parent" and "foster child" in preference to "looked after child" (the British term for foster child), "carer" and "caregiver". I use these latter terms if they are used in a study I am describing. The term "mental disorder" is used in preference to "psychiatric disorder" and "mental illness", whenever prevalence rates are described, and the more general "mental health problems" is used at other times. The statutory agency charged with the care of foster children in New Zealand is Child, Youth and Family. I have used this term when describing New Zealand statistics and studies and, more generally, I have used the terms child welfare or child protection. Māori are the indigenous people of New Zealand and constitute $15 \%$ of the population but about half of the children in foster care. The Māori word for family is "whānau" and is in general usage in Child, Youth and Family publications as "family/whānau" and I have used this term when referring to New Zealand statistics.

\section{The Context of Foster Care}

\section{Foster Care in New Zealand}

Estimating from the number of maltreatment notifications and number of children in foster care in 2014 , less than one in five children with substantiated maltreatment enter foster care. The primary reason for a child's placement in care after substantiated maltreatment is concern about the child's ongoing safety (Child, Youth and Family, 2014). Other reasons 
children may enter statutory care include removal from parents at birth, usually after substantiated allegations of maltreatment of older children in the family, parent incapacity or death. In western countries foster care is seen as the main public health intervention for maltreatment. The number of New Zealand children in the care of the state increased markedly after the second world war, with a peak of 5,515 in 1971-1972 (Pollock, 2013). In contrast, the number of children in foster care in recent years has been rapidly decreasing from 5,095 in 2006 to 4,129 in 2014, indicating a 19\% decrease in the last 8 years (Child, Youth and Family, 2006, 2014). This contrasts with a marked increase in maltreatment notifications, which will be discussed later.

Under the Children, Young Person's and their Families Act 1989, social workers in New Zealand are required to explore all avenues for alternative care within the child's family or whānau, largely due to concerns about the number of Māori children placed with nonMāori families (Murray, Tarren-Sweeney, \& France, 2011). Of the 4,129 children who were in out of home care in New Zealand in June, 2014, 48\% were with a family or whānau caregiver, $31 \%$ were with non-kin foster parents, $13 \%$ had family support services, and almost 4\% were in group homes or residences (Child, Youth and Family, 2014). By ethnicity, $56 \%$ were Māori, $32 \%$ were NZ European and $8 \%$ were Pacific Islanders.

Children in foster care may remain in care for a substantial amount of time, many return to care at a later date and some children experience considerable placement instability. In 2006, almost half of the children in foster care remained in care for at least two years, a third of young people who left care returned at a later date, and $10 \%$ of children had experienced more than eight placements (Child, Youth and Family, 2006). In 2010, the range of time that foster children had spent in their current placement was $0-9$ years, with an average duration of just under a year (Atwool, 2010). In relation to placement changes, about half of the children (52.4\%) had between one and three caregivers, about a quarter (25.5\%) had four to six caregivers and $22.1 \%$ had more than six caregivers. In addition, children aged between 14 and 16 years in care and protection residences had an average of 14 previous placements. Taken together, these figures suggest children in care have considerable placement instability.

Parents of children in care. Many of the parents of children in care have mental health and substance abuse problems and had contact with Child, Youth and Family themselves as children. Connolly, Wells and Field (2007) found that almost half (49\%) of the mothers of children under two years of age who entered care, and $41 \%$ of the fathers, had had child welfare involvement when they were young. Seventy one per cent of the mothers 
had drug and alcohol problems, $43 \%$ had mental health problems, $25 \%$ had criminal convictions and $10 \%$ had an intellectual disability (Connolly et al., 2007; Rankin, 2011).

Inter-country comparisons. New Zealand has a comparable rate of children in foster care to other developed countries. In a study of trends in Australia, Holzer and Bromfield (2008) found rates of 4.8 per 1000 children in New Zealand and 4.9 per 1000 in Australia, in contrast to higher rates in the US (7.1 per 1000) and lower rates in Canada (2.8 per 1000). However, the rate in Australia rose sharply to 7.7 children per 1,000 in 2012 (Australian Institute of Health and Welfare, 2013). This contrasts with the current rate for the United States (5.1 per 1000).

The New Zealand care system can be differentiated from those in North America and the United Kingdom by the relatively low use of residential care, the high use of kinship care and the absence of an adoption from care provision within legislation (Murray et al., 2011). Murray et al. note the move to family/whānau care was due to "increased sensitivity to the cultural and identity needs of indigenous children, who are over-represented among children in care" (p. 151). A recent initiative called "Home for Life" has been implemented which aims to increase the placement stability of children unable to return home (Ministry of Social Development, 2012).

\section{Disproportionate Indigenous, Minority and Poor Children in Care}

Māori children had little contact with child welfare until the 1940s, but the numbers of Māori children in residential care increased in the 1960s (Pollock, 2013). Māori had a traditional system of whāngai, where relatives or other members of an iwi (tribal group) would look after children whose parents had died or were unable to support them. It was also traditional for grandparents to whāngai a child whose parents were able to care for them, for the purpose of passing on traditional knowledge (Keane, 2014). Whāngai was openly acknowledged and the child knew the identity of their birth parents. Although the adoption act of 1955 legislated for secrecy around adoption, Māori continued with the practice of whāngai (Keane, 2014).

In most countries, there is a disproportionate number of indigenous and minority children in foster care. In New Zealand, there are over three times the number of Māori children in foster care than their numbers in the population would suggest (approximately 15\%). Many of these children are fostered by relatives. The number of Māori children being brought up in non-Māori homes is not known. In Australia, Holzer and Bromfield (2008) report that the rate of placement for Aboriginal and Torres Strait Islander children is 55.1 per 
1000, compared to an expected rate of 4.72 per 1000, and a non-indigenous rate of 5.4 per 1000. However, over two thirds of indigenous children are placed with relatives or kin, other indigenous caregivers or in an indigenous residential care facility (Australian Institute of Health and Welfare, 2013).

Before 2002, African-American children in foster care had over three times the rate of placement of White children, but this rate has significantly declined to twice the rate (9.6 per 1000 compared to 4.6 per 1000). The highest rates in the US are now for Native American children, at 13 per 1,000 (U.S. Department of Health and Human Services, 2013). There appears to be a more even representation across race in the UK, in that $74 \%$ of the children in care and $87.1 \%$ of the general population are White British.

There are differing explanations for the over-representation of indigenous and minority children in foster care. Some authors have argued that the overrepresentation of minority and indigenous children in foster care is an example of discrimination, and that social workers are more likely to place minority and indigenous children than White children in the same circumstance (Miller, Cahn, Anderson-Nathe, Cause, \& Bender, 2013). The disproportionate numbers of indigenous and minority children in foster care have also been attributed to the levels of poverty and disadvantage in those communities. In the US, the emergence of widespread homelessness, substance abuse, unemployment, and welfare reform have all impacted poor communities (Conn et al., 2013; Franz \& Woodward, 2006). Entering the child welfare system is associated with poverty and about half the children in foster and residential care come from families which have difficulties meeting basic needs (Barth, Wildfire, \& Green, 2006). Children may also enter foster care for mental health treatment, but the rate at which this occurs has not been established (Barth et al., 2006). Simkiss, Stallard and Thorogood (2012) examined the factors associated with children entering care. They found that entry into care was associated with a maternal history of disadvantage, such as low socio-economic status, younger maternal age at birth, alcohol abuse, mental health problems, and with child factors such as low birth-weight, disability, injuries, and attendance at emergency rooms.

Research has investigated the effects of children having foster parents of the same or different culture. African American children have been found to have fewer internalising problems when fostered by same race families (Anderson \& Linares, 2012). In the Casey Field Office study, $43 \%$ of minority youth said it was hard for youth in foster care to develop their ethnic identities and 69\% said they would like to learn more about their ethnic identities (White, Havalchak, Jackson, O’Brien, \& Pecora, 2007). If the minority youth had foster 
parents who helped them to develop their ethnic identities they were 1.6 times less likely to develop depression (White et al., 2009). Some studies have shown that African American children are less likely than children of other races to receive services when there is clinical need (Burns et al., 2004; Stahmer et al., 2005).

In summary, minority and indignenous children are placed in foster care at higher numbers than other children and reasons may include social workers being influenced by race as well as the poverty in these communities. Several countries have attempted to change the situation by making more use of kinship care and proportions of minority and indigenous children in non-kin foster care have recently dropped, particularly in the US.

\section{Child Maltreatment in New Zealand}

Almost all of the children who come into foster care have been maltreated. In this chapter, I consider the prevalence of child maltreatment and selected psychological and neurobiological effects, as maltreatment is thought to be critical factor in foster children's mental health. Child maltreatment encompasses physical abuse, sexual abuse, emotional abuse and neglect (Cicchetti \& Valentino, 2006). Three longitudinal, population based studies in New Zealand have yielded a great deal of data about child maltreatment. Severe maltreatment, including being exposed to frequent and severe punishment or harsh and abusive treatment, was reported by $6 \%$ of the participants in the Christchurch Health and Development Study and $8.5 \%$ of participants in the Dunedin Multidisciplinary Health and Development Study (Breslau et al., 2014; McLeod, Fergusson, \& Horwood, 2013). Moderate maltreatment was reported by $27 \%$ of the Dunedin sample and $46 \%$ of the participants in the Youth 2000 study had witnessed adults yelling or swearing at a child, with 17\% witnessing adults hitting or physically hurting a child (Breslau et al., 2014; Clark et al., 2009).

According to international agency reports, New Zealand has a high rate of family violence. The Organisation for Economic Cooperation and Development (OECD) has shown that New Zealand had the the fifth highest rate of child maltreatment in the OECD in the early 2000s and the highest rate of reported partner physical or sexual assault in the OECD in 2005 (Organisation for Economic Cooperation and Development, 2009, 2013). A large number of children are present at family violence incidents and offences, including 65,159 children in 2005 (Every Child Counts, 2010). A UNICEF Innocenti report showed that New Zealand is in a band of countries with six times the rate of deaths from child maltreatment compared to the countries with the lowest rates (UNICEF, 2003). According to the Every 
Child Counts report, child maltreatment costs New Zealand approximately $\$ 2$ billion per year, which is $1 \%$ of our Gross Domestic Product. This includes direct costs of child protection and health services, long term health consequences, crime impacts and indirect costs in terms of lost productivity (Every Child Counts, 2010). It has been noted that New Zealand may detect more maltreatment than other countries due to the comprehensive systems for collating nationwide police reports of incidents involving children, and the presence of social workers in schools, which may enable better detection (Gilbert et al., 2012).

Notifications to child protection services have increased exponentially in New Zealand over the past 30 years. While notifications of possible maltreatment received by Child, Youth and Family have increased from about 2000 reports of concern per year in the 1980s to 146, 657 reports of concern in 2014, fewer than half of these referrals were deemed to require further action (Child, Youth and Family, 2014). The increase in reports may partly be a result of public awareness and partly a result of the police reporting all children present at domestic violence incidents (Child, Youth and Family, 2014; Rankin, 2011). Of the care and protection reports of concern, $61 \%$ were followed up and $36 \%$ were substantiated. The types of abuse were: emotional abuse (56\%), neglect (24\%), physical abuse (15\%) and sexual abuse $(6 \%)$.

In summary, research has shown that between 6 and 9\% of New Zealand children experience severe maltreatment. Rates of moderate maltreatment or witnessing abuse are between $27 \%$ and $46 \%$. International comparison studies have shown high rates of maltreatment and child death in New Zealand, although these figures may be affected by the rigour of systems and reporting in each country. With public campaigns about reporting child abuse, notification rates are very high, but fewer follow-up and substantiation rates are considerably lower.

\section{Selected Effects of Child Maltreatment on Socio-emotional Development}

Many children in foster care have experienced several types of maltreatment over an extended period of time. Maltreatment is compounded by birth parents of children in foster care having high levels of substance abuse, mental health problems, domestic violence and poverty (Connolly et al., 2007; Oswald, Heil, \& Goldbeck, 2010). Maltreatment is known to affect children's ability to regulate their emotions, their level of internalising and externalising problems, suicidal thoughts and actions and the development of a secure attachment, with a high number of maltreated children having disorganised attachment 
(Cicchetti \& Toth, 2014; Cicchetti \& Valentino, 2006). Maltreatment has been described as "a toxic relational environment that poses considerable risk for maladaptation across diverse domains of development" (Kim \& Cicchetti, 2010, p. 706).

Effects on attachment and emotional regulation. The effect of maltreatment on children's emotional regulation and attachment security is well-established. Maltreated children are likely to come from homes characterised by high levels of negative affect and low levels of emotional support (Fonagy, Gergeley, \& Target, 2007; Shipman et al., 2007). Shipman et al. showed that maltreated children have poorer emotional regulation and more emotional dysregulation than non-maltreated children. Moreover, maltreating mothers supplied less emotional support and validation to their children. Shipman et al. argued that this may be because maltreating mothers have more difficulty managing their own emotional arousal when dealing with their children's negative emotions. Other research demonstrates emotional dysregulation in maltreated children as young as 3 months (Cicchetti \& Valentino, 2006). In addition, maltreated children have been shown to have less accurate emotion recognition, particuarly in relation to anger, and less ability to regulate behaviour in response to adult anger (Luke \& Banerjee, 2013).

Many maltreated children have a disorganised pattern of attachment which is thought to develop when children are unable to develop a clear strategy to respond to a parent who is abusive and fear-provoking (van IJzendoorn, Schuengel, \& Bakermans-Kranenburg, 1999; see also Chapter Three). Disorganised attachment has been linked to the development of subsequent psychopathology and maltreatment has been found to be one of the strongest predictors of disorganised attachment (van IJzendoorn et al., 1999). Children with disorganised attachment are more likely than children with organised attachments to develop externalising behaviour problems (Fearon, Bakermans-Kranenburg, van IJzendoorn, Lapsley, \& Roisman, 2010; van Ijzendoorn et al., 1999). Maltreatment also affects children's mental representations or ideas about interpersonal relationships and this research is presented in Chapter Three, along with the research findings on disorganised attachment in foster children.

Effects on neurobiology. Early maltreatment and out of home care has been found to have neurobiological effects on children's cortisol levels, Hypothalamic Pituitary Adrenal (HPA) axis, oxytocin and vasopressin levels, and on functioning of various brain structures (Toth, Gravener-Davis, Guild, \& Cicchetti, 2013). The results of neurobiological studies have lead researchers to conclude that parents or caregivers act as a buffer to mediate high levels of stress in young children (Fisher, Gunnar, Dozier, Bruce, \& Pears, 2006; Fonagy, 
Gergely, \& Target, 2007). For children in out-of-home care, various abnormal patterns of cortisol have been found. This is in contrast to typically developing children whose cortisol level is low in the morning and peaks at mid-afternoon. Studies of young maltreated children in care have found both high and low levels of cortisol. Importantly, these patterns normalised after caregivers were trained to be more responsive (Dozier et al., 2006). Recent studies have shown that high cortisol levels are associated with internalising behaviours such as anxiety and depression, and low levels are related to externalising problems (Laurent, Gilliam, Bruce, \& Fisher, 2014).

For maltreated children in care, the lack of a responsive parent and/or separation from a parent may be particularly harmful. Animal studies have shown that when rodent pups are separated from their parents, there is a critical period, known as the Stress Hypo-responsive Period. In this period, the animal's HPA axis, which is activated in response to stressors, is less able to modulate stress and an outside modulator, such as a caregiver or parent, is needed for this to occur (Fisher et al., 2006). These authors argued that "the absence of a responsive, supportive caregiver to serve an external extension of the child's stress regulatory system might have the most pervasive long term effects on the child" (italics in original, Fisher et al., 2006, p. 219). This work has recently been extended into the field of epigenetics or gene by environment interactions. For example, a recent study has shown that variations of the Monoamine Oxidase A gene moderates the relationship between a history of adversity in childhood and criminal offending (Fergusson, Boden, \& Horwood, 2012)

In summary, in addition to the well-established finding that maltreatment affects children's mental health, recent studies have shown that maltreatment is associated with disorganised attachment, in itself a risk for mental health, emotion regulation, and atypical cortisol patterns. It has also been increasingly understood that parents buffer stress for young children and that lack of, or separation from, a responsive cargiver is thought to be very stressful for young children.

The overarching goal of this thesis is to examine whether foster parents' focus on children's mental life and emotions assist children who have the difficulties described above, and whether assisting foster parents to more fully consider the child's history, which usually includes maltreatment, is helpful. In the final section of this chapter, I outline the aims and structure of the thesis. 


\section{Aims and Structure of the Thesis}

The first overall aim of this thesis is to examine whether foster parent's awareness of their foster child's mental life and emotions, conceptualised here as mind-mindedness, is associated with positive outcomes for the child. The second overall aim is to examine whether mind-mindedness can be enhanced in both foster parents and foster care practitioners, through a training programme which focusses on understanding the foster child's perspective.

The aim of the first study is to examine the key predictor of foster parent mindmindedness and a range of other predictors, including child characteristics, placement characteristics and aspects of the foster parent's psychological functioning, on foster children's emotional and behavioural problems and the quality of the parent-child relationship. Prior research suggests three aspects of foster parent's psychological functioning may be critical: a) foster parent's mind-mindedness as outlined above, b) foster parent's perceptions of their own attachment relationship with their primary caregiver, both in the past and currently, and c) the foster parent's emotional availability to the child. As far as is known, this study is the first to examine the role of foster parent's mental representations or mind-mindedness.

The second study employs a multiple-baseline design to examine whether foster parent mind-mindedness increases after an intervention focussing on understanding the child's thought and emotions. A Relational Learning Framework (RLF) and training programme were developed by the author to assist foster parents to understand the child in their care and to use this understanding to discover what the child needs to learn in current relationships (Kelly \& Salmon, 2014). The RLF teaches foster parents how children's behaviour problems can provide indicators of what they may have learned about relationships from their previous adverse experiences. For example, if a child has been abandoned by biological parents, and subsequently finds it difficult to separate from foster parents, this behaviour may provide clues that the child may fear the foster parent is also going to leave them. However, a child with a similar experience of being abandoned by biological parents who runs away, may have learned that families are not a safe or rewarding place to be. These symptomatic behaviours can be integrated with knowledge of the child's history to help the foster parent understand the child. This understanding can then be harnessed to help the child to gradually form a more positive view of themselves and relationships with others. The third study investigates 
whether this intervention is useful for practitioners to learn and apply in their work with foster children and families.

Inclusion of studies in the thesis. As only a small number of studies use large, representative samples of children in foster care, most reviews of foster care include data from children with open child welfare files (e.g. Burns et al., 2004, Garland et al., 2001). They also include children who have been adopted, as about one third of these children have a foster care history, at least in the US and the UK (Carnes-Holt \& Bratton, 2014; Biehal, Ellison, Baker et al., 2011). Conversely, studies of child maltreatment often provide information about children with open child welfare files, children in foster care or adults who have a history of either (e.g., Scott, Smith, \& Ellis, 2010). For these reasons, studies have been included of children with child welfare involvement, children who have been adopted and adults who have a history of any of these factors.

A number of studies focus on the correlates of successful placements, particularly placement stability. These studies are included as placement stability is seen as critical to children's mental health outcomes. Studies considered beyond the scope of this thesis, and therefore excluded, are those focusing on kin versus non-kin placements, family reunification, the perceived burden of foster care on carers, the importance of contact with birth parents, whether sibling groups should be placed together, maltreatment while in foster care, and foster children's and parents' views on foster care. These issues are included in the thesis in relation to the child's mental health only and are not covered as topics in their own right.

Structure of the thesis. The first chapter of the thesis introduced the topics of child maltreatment and foster care in New Zealand, with comparisons to other countries. The second chapter includes the prevalence and type of mental health problems foster children have and the mental health factors related to placement disruption. Chapter Three covers the broad theoretical framework used for studying the effects of maltreatment and foster care and the main theoretical concepts used in the three studies, which are attachment, mindmindedness and emotional availability. The fourth chapter outlines the first study which examines which psychological characteristics of foster parents are associated with children's emotional and behavioural problems and the quality of the foster parent-child relationship. Chapter Five reports the literature on the effectiveness of interventions with foster parents and children. The sixth chapter introduces the Relational Learning Framework (RLF) I have developed to help foster parents and foster care practitioners understand the child in their 
care. The chapter consists of an article published in Clinical Child Psychology and Psychiatry (Kelly \& Salmon, 2014).

Chapter Seven and Eight evaluate the effectiveness of a training programme incorporating the RLF. The second study employs a multiple-baseline design to evaluate the training with foster parents. The third study employs a pre- post-test design with foster care practitioners. The overall discussion and conclusion chapter then presents the results, their implications for practice and further research, and the strengths and limitations of the studies. 


\section{Chapter Two-Foster care: Child mental health and outcomes}

Many children in foster care report satisfaction with their placements and are considered to be in successful placements (e.g., Wilson, 2006). However, the high rates of mental health problems in foster children and adolescents, combined with the lack of an overall positive effect of foster care placement on children's mental health, suggests that foster parents may be an under-utilised resource for assisting foster children to overcome their considerable social and mental health difficulties. The current chapter presents the literature regarding the wellbeing of children and adolescents in foster care, their developmental and mental health problems, and the impact of both multiple placements and foster care itself.

\section{Foster care and general well-being/resilience}

Many foster children report feeling satisfied with their foster family and studies have shown that the majority of placements are successful (Schofield \& Beek, 2005b; Wilson, 2006). For example, in South Australia, 48 children and adolescents in care were interviewed about their satisfaction with their placements and over $90 \%$ indicated they felt happy and safe with their foster family, had fun with them, and felt wanted and supported (Barber \& Delfabbro, 2005). Only 6.3\% felt that they needed more help than they received. A study involving 47 children in foster and residential care in New Zealand found that the great majority of children $(91.5 \%)$ rated themselves at 5 or above out of 10 on a rating scale where zero was not happy and 10 was very happy with their life (Atwool, 2010). When asked what was good about being in care, $21 \%$ mentioned financial support, others said they were well looked after and some said they had turned their life around. When asked what was bad about being in foster care, a quarter said not being with their family, others said not being treated fairly or being stigmatised, and $8.5 \%$ said being abused or treated badly in some placements. A small percentage thought there was either "nothing good" or "nothing bad" about foster care.

There is also some evidence of the resilience of foster children. In a study of 193 foster children in one local authority in Wales, $16 \%$ of the children met the criteria for what the study termed "positive exceptions" (Rees, 2013, p. 183). This was defined as having a total score on a broad band measure of psychological functioning (Strengths and Difficulties Questionnaire; Goodman \& Scott, 1999) in the average range, literacy in the expected range for ability, and $85 \%$ attendance at school. Almost half of the children (47\%) were in the average range on a measure of cognitive ability, and just over $40 \%$ were average or above for 
literacy. In a study of in 531 children aged between 5 and 9 years in foster care, Bell, Romano and Flynn (2013) examined the prevalence of resilience in foster children. They defined resilience as normative functioning on the emotion, conduct, and pro-social subscales of the Strengths and Difficulties Questionnaire (SDQ; Goodman \& Scott, 1999), despite exposure to adversity. Thirty per cent of the children were in the normative range for all three behavioural outcomes. When academic performance and peer relationships were added to behavioural outcomes, however, only $7 \%$ of the children were resilient on all five outcomes.

In summary, a majority of children in foster care describe their current placement in positive ways and a minority of children in care can be seen as resilient, at least in some areas of cognitive, emotional or behavioural functioning.

\section{Foster care, Maltreatment, Physical health and Social Outcomes}

Children in foster care have high rates of physical health problems, as well as mental health problems (Anctil, McCubbin, O'Brien, \& Pecora, 2007) and it is likely that these are related to the adverse environments that foster children have experienced. It has increasingly been recognised that adverse childhood experiences such as maltreatment lead not only to higher mental health risks, but also to a variety of physical health and social risks, particularly in adulthood. The Adverse Childhood Events study (ACE) has shown that traumatic stressors in childhood are strongly linked to many negative health behaviours and outcomes. When participants were followed up, those with six or more adverse events died 20 years earlier, on average, than those without any adverse events (Brown et al., 2009). Adverse events included physical or sexual abuse, neglect, parent substance abuse or domestic violence in childhood. The authors noted that this risk of premature death risk was only partly explained by the documented health and social problems associated with adverse childhood events.

The Dunedin Multidisciplinary Health and Development Study examined the relationship between adverse childhood experiences, including child maltreatment, and two indicators of physical health as adults (Danese et al., 2009). Adults who had been maltreated as children had higher levels of chronic inflammation, which is known to be a predictor of age-related diseases. The authors concluded that this increase in age-related health risk could not be attributed to current lifestyle risk predictors, such as smoking and poor diet, and that “...children exposed to adverse psychosocial experiences have enduring abnormalities in multiple biological systems" (Danese et al., 2009, p. 1141). 
Adults who have been in foster care as children have low rates of participation in tertiary education and high rates of teenage pregnancies, unemployment, homelessness, and incarceration (Berzin, 2008). Of adults who have a Department of Corrections sentence in New Zealand, $67 \%$ have been in foster care and just under a third of children (29\%) who have contact with child welfare progress to a corrections sentence (Ministry of Social Development, 2011). Young people leaving foster care are expected to be living independently as young as $17-18$ years in most jurisdictions. There are few transition programmes for youth in New Zealand, although this has been identified as an issue to be remediated (New Zealand Government, 2012).

\section{Foster Care and Developmental Problems}

Substantial numbers of children in care have been shown to have developmental problems, which can be defined as any ongoing delay to a child reaching age specific milestones (Eapen, 2014). These may be attributable to multiple factors, for example: genetic factors, in utero exposure to drugs and alcohol, poor nutrition of the child's birth mother and during the early years, as well as the experience of maltreatment and lack of stimulation (Belsky, Bakermans-Kranenburg, \& van IJzendoorn, 2007; Caspi \& Moffitt, 2006; Cicchetti, 2010).

Pre-schoolers in foster care have been found to have delays on measures of height and head circumference, language, general intellectual functioning and visuospatial functioning, compared to a group of matched, non-maltreated children (Pears \& Fisher, 2005). The level of neglect the child had experienced was negatively associated with the child's level of functioning. Comparable rates of developmental delay were found in a study using a nationally representative US sample in the National Survey of Child and Adolescent Wellbeing (NSCAW) study. Just over a quarter of the children under two years and $13.6 \%$ of the three to five year old children had a developmental or cognitive delay as measured by a standardised intelligence test, with only $22.7 \%$ of the children receiving assistance for this (Stahmer et al., 2005). Older children and adolescents in foster care in the UK were also found to have high rates of developmental delay: $13 \%$ of the sample had a neurodevelopmental disorder, $11 \%$ had a mental age $60 \%$ or more below their chronological age and $23 \%$ of the children had a statement of special needs (Ford, Vostanis, Meltzer, \& Goodman, 2007). These rates were all significantly higher than the rates for children in the general population in disadvantaged households. 
In summary, although it is difficult to compare studies using different measures and samples, a substantial minority of children in foster care have been found to have significant developmental delay and to perform more poorly on tests of general intellectual functioning than children matched on demographic variables, who have not been maltreated.

\section{Prevalence of Mental Health Problems: Studies including both Foster Children and Adolescents}

The prevalence of mental health problems in children and adolescents in foster care has been widely studied but the predictors of poor mental health in foster children have received less attention. Because of the considerable research, the focus of the current chapter is the findings of large, nationally representative samples, where available.

Remarkably similar results have been found in studies of the prevalence of mental health problems in foster children and adolescents, despite the different measures, age groups and countries involved. Forty to fifty per cent of foster children and adolescents show rates of clinical problems or mental disorders (Burns et al., 2004; Ford et al., 2007; Meltzer, Gatward, Corbin, Goodman, \& Ford, 2003; Steele \& Buchi, 2009). For example, 48\% of children aged between 2 and 14 years with a child welfare file in the US scored within the clinical range on the Child Behaviour Checklist (Achenbach \& Rescorla, 2000; Burns et al., 2004). Similarly, $42 \%$ of youth aged between 6 and 18 years with a child welfare file met criteria for a Diagnostic and Statistical Manual of Mental Disorders Diagnosis (DSM IV; American Psychiatric Association, 2000) diagnosis (Garland et al., 2001).

Similar findings have been reported in UK studies. The Department of Health carried out a survey of the mental health of foster children aged between 5 and 17 years using a structured diagnostic interview and found $45 \%$ of the children had a mental disorder (Meltzer et al., 2003). This rate was four to five times the rate of mental disorder of children living with their own families (Meltzer et al., 2003). The authors concluded that "Looked after status [foster care] had the strongest association with disorders in which environmental factors are believed to have a leading role, such as post-traumatic stress disorder and conduct disorder" (p. 323). Particularly at risk were boys, older children, children who had been in care less than a year, and those in residential care.

In New Zealand, most prevalence data involves adolescents, but a small study of health and education assessments for 94 children entering care found that $65 \%$ had an emotional or behavioural problem and $41 \%$ had a mental disorder (Rankin, 2011). In Australia, just over half of the children in a large sample of 347 children ages 4 to 11 years had scores within the 
clinical range on one Child Behaviour Checklist sub-scale (Tarren-Sweeney, 2008a). Subscale means were also between 0.3 and 1.9 standard deviations above the Australian general population means.

In summary, a number of studies across a range of countries, age groups and measures have found that around $40-50 \%$ of children in foster care have rates of mental health problems which are at least two to three times higher than the rate for children in the community.

\section{Type of mental disorder}

Almost all studies of mental health problems in foster care have found much higher rates of externalising behaviour, including conduct problems, compared to internalising problems. Rates of externalising behaviour, including conduct problems and/or attentional problems, are consistently found to be $35-40 \%$ across a range of countries, including New Zealand (e.g. Burns et al, 2004; Meltzer et al, 2003, Wells, 2004). Rates of internalising problems are considerably lower at around $10-15 \%$. For example, in the UK, $12 \%$ of the children in foster care experienced anxiety or depression (Meltzer et al., 2003). The authors concluded that "...conduct disorders seem to contribute to the largest difference in childhood psychopathology between local authority and private household populations" (Meltzer et al., 2003, p. 21).

It is possible that studies have found low rates of internalising problems, because these are less evident to foster parents who are reporting the child's mental health problems. However, studies including children aged 11 years and older have included the children as informants, either with a structured interview or a youth self-report measure (e.g. Burns et al., 2004; Meltzer et al., 2003). This suggests that under-reporting is less likely, although children who have been in adverse environments may also be less adept at reporting their own internal states. In addition, some studies have found higher rates of internalising problems. For example, in a high risk group of 43 pre-schoolers in foster care, there was a higher prevalence $(23 \%)$ of internalising problems and $33 \%$ of the children were diagnosed with reactive attachment disorder (Hillen \& Gafson, 2014). A Norwegian study of foster children ages 5 to 12 years, also found a higher rate (24\%) of emotional disorders and $19 \%$ of the children had Reactive Attachment Disorder (Lehmann, Havik, Havik, \& Heiervang, 2013). Other studies have also found that foster children have higher rates of Reactive Attachment Disorder than children in the general population (e.g., Minnis, Everett, Pelosi, Dunn, \& Knapp, 2006). This is not surprising as one of the criteria for Reactive Attachment 
Disorder is that children have had extremes of poor care (American Psychiatric Association, 2013).

Further evidence of internalising problems are the highly elevated rates of suicidal thoughts and actions occur among foster children from an early age. Twenty six percent of children aged between 9 and 11 years, who were in foster care after maltreatment, had a history of suicidal thoughts or actions (Taussig, Harpin, \& Maguire, 2014). This is five times the rate in the general population. In addition, $4.1 \%$ of these children were imminently suicidal and the foster parents of two thirds of these children were unaware of the problem. The most robust predictors of suicidality were physical abuse and the chronicity of maltreatment. Children had a higher likelihood of attempted suicide when they had spent longer in out of home care but the length of time with current caregiver was unrelated.

About a third of children in foster care $(35 \%)$ have been shown to have non-complex clinical problems, consistent with a discrete mental disorder, and $20 \%$ have been found to have complex attachment and trauma symptoms (Tarren-Sweeney, 2013b). Tarren-Sweeney argued that these complex problems are not adequately conceptualised in current diagnostic systems.

It is widely accepted that foster children have a predominance of externalising behaviour problems, but recent studies using structured interviews and questionnaires which include Reactive Attachment Disorder and suicidal behaviour, have shown significant internalising problems in foster children and adolescents. This in unsurprising given the multiple losses of family and social connections that many foster children experience (Unrau, Seita, \& Putney, 2008).

\section{Correlates and predictors of mental health problems}

Multi-level and cumulative risk studies. Limited attention has been paid to the correlates of foster children's mental health. While many studies have examined the predictors of children being placed in care and placement disruption, studies of foster children's mental health have focused on prevalence. Most studies have used cross sectional designs to focus on only one or two variables, in addition to basic demographic variables. Statistical techniques, such as regression analysis and structural equation modelling, have not been applied in many of these studies, as prevalence is the focus (e.g., Burns et al., 2004; Meltzer et al., 2003). An exception to the prevalence approach are studies using multi-level modeling to examine the impact of child, family, practitioner, and welfare agency variables 
on children's outcomes (Bell et al., 2013; Cheung, Goodman, Leckie, \& Jenkins, 2011). For example, Bell et al. included variables at the child level, such as maltreatment and contact with birth parents, family-level variables such as self-reported parenting skills, and workerlevel variables such as child welfare experience and agency-level variables such as caseload. Most variance in behavioural outcomes was explained by child variables, followed by family variables, particularly ineffective parenting. Interestingly, worker- and agency-level variable also contributed a significant but small amount of variance.

In the most comprehensive study to date, Tarren Sweeney (2008a) found the strongest predictors of the child's poor mental health were older age at entry to care, developmental factors (reading difficulties and foster parent reported intellectual disability), younger maternal age at birth of child, specific types of maltreatment, foster parent's expectation that the child would return home and more adverse events in the past year. Developmental factors such as intellectual and reading difficulties and the child's history prior to being placed in foster care accounted for about two thirds of the variance that the model explained. Prior adversity was most closely related to attachment difficulties, aggression, defiance and age inappropriate sexual behaviour.

In the first study on cumulative risk in foster care, seven out of eighteen risk factors significantly predicted mental health problems in children aged 9 to 11 years (Raviv, Taussig, Culhane, \& Garrido, 2010). Risk factors included physical and sexual abuse, number of caregiver and school transitions, low intellectual functioning, having a single parent and exposure to community violence. Evidence of a threshold effect above which risk of mental health increased dramatically, as proposed by Rutter (1979), was not found, but there was evidence of an incremental increase in cumulative risk.

In summary, while studies included different variables, common findings include the impact of the child's age or age of entry into care, the child's intellectual functioning, the extent of maltreatment of the child and foster parent expectations and functioning.

Child maltreatment, trauma and mental health. The link between child maltreatment and mental health problems has been well establshed (Cicchetti \& Valentino, 2006; Fergusson, Horwood, \& Lynsky 1996). There have been mixed findings about the impact of the specific type of abuse, number of different types of abuse, severity and chronicity on the development of mental disorders in foster children (English et al., 2005; Lau et al., 2005; Litrownik et al, 2005). In some studies, multiple types of abuse are a robust predictor of children's mental health outcomes but specific types of abuse, severity and frequency of abuse also contribute unique variance to some aspects of the child's functioning 
(Lau et al., 2005). One study found that severity of abuse, but not frequency of abuse, was related to externalising behaviour and adaptive functioning in foster children and youth (Jackson, Gabrielli, Fleming, Tunno, \& Makanui, 2014). In the UK, rates of mental disorder for children who had been abused or neglected were found to be $62 \%$, compared to $10 \%$ of children who were in care due to family dysfunction (Meltzer et al., 2003).

It is possible that trauma is at least a partial explanation for the elevated rates of mental health problems in foster care. The link between maltreatment and externalising behaviour problems was partially accounted for by trauma in a study of at risk pre-schoolers (Milot, Ethier, St-Laurent, \& Provost, 2010). The authors noted “ ...the importance of understanding psychosocial maladjustment of maltreated children not only from the perspective of problematic behavior, but also by taking into account the traumatic reactions that might develop in response to chronic and intense stress associated with abuse and neglect (Milot et al., 2013, p. 225).

Age differences. Older children have been shown to have higher rates of mental health problems than younger children, but this finding may be better accounted for by the age at which the child entered care (Tarren-Sweeney, 2008) . Risk of mental health problems rises in children entering care over the age of 6 to 7 months (Hillen \& Gafson, 2014; TarrenSweeney, 2008a). The magnititude of contrast between the rates of mental health problems between younger and older children may be quite large. One study found that as many as $66 \%$ of children aged between 11 and 14 years with child welfare files have significant clinical problems, compared to 32.2\% of preschoolers (Burns et al., 2004). Similar rates were found in a large sample of 6177 children in foster care (Steele \& Buchi, 2009). While $44 \%$ of all of the children had one or more mental health problem, the prevalence for children over 12 years was $68 \%$. The effect of foster children's age on their mental health may be explained by older children entering care at an older age, experiencing more placement disruption or being more aware of their loss of family connection and their social circumstances (Nutt, 2006; Tarren-Sweeney, 2008a).

Gender differences. Gender differences are found on some types of measures and not others. Standardised measures such as the Child Behaviour Checklist (CBCL) or the Strengths and Difficulties Questionnaire (SDQ) do not generally show gender differences (e.g., Burns et al., 2004; Tarren-Sweeney, 2008). However, gender differences are usually found for discrete disorders. In one study, there was no gender difference in overall rates of disorder but boys had higher rates of attention deficit and hyperactivity disorder (ADHD) and conduct disorder while girls had more post-traumatic stress disorder (PTSD), major 
depression and separation disorder (Garland et al., 2001). In contrast, in a UK study, boys were twice as likely to have any disorder as girls (Ford et al., 2007). These different rates of disorders for boys and girls in foster care reflect rates found in the general population (Mash \& Wolfe, 2013).

In summary, the magnitude of gender differences in foster children's mental health are more evident when research involves structured interviews assessing discrete disorders, rather than broadband questionnaires.

\section{Prevalence of mental health problems in samples of adolescents only}

The rates of mental health problems in the general population rise sharply in adolescence (Fergusson \& Horwood, 2001; Fergusson, et al., 1996). It is important to identify whether adolescents in foster care continue to have rates of mental health problems which are markedly above their peers in the general population. The evidence is contradictory. First, two studies have found that rates of lifetime mental and substance use disorder, and past year mental and substance abuse disorder, in adolescents in foster care are markedly higher than those of community samples (Aarons, Brown, Hough, Garland, \& Wood, 2001; McMillen et al., 2005). Second, one study found that adolescents in foster care have similar rates of past year diagnoses to adolescents in the general population, but have much higher rates of lifetime diagnoses (White et al., 2007). For example, in the the National Casey Field Office Study, 36\% of both youth in foster care and youth in the general population had a past year diagnosis, but $63 \%$ of foster youth had lifetime diagnoses, compared to $46 \%$ of the general population (White et al., 2007). White et al. (2007) also found that foster youth had approximately three times the rate of attention deficit and hyperactivity disorder and conduct disorder and two and a half times the rate of posttraumatic stress disorder, compared to the general population. Third, the Midwest study of 737 current and former 17 year old foster youth found that both lifetime and past year diagnosis rates of mental and substance use disorders were comparable to those of the general population, with the exception of PTSD (Keller, Salzar, \& Courtney 2010).

Studies of New Zealand foster youth have found markedly high rates of mental health problems. Research has shown, first, that young people with histories of contact with Child Youth and Family have double the rate of at least one mental disorder diagnosis (80\%) compared to those without welfare contact (40\%; Smith \& Beautrais, 1999). Second, a quarter of the 61 children under the age of 15 years who died by suicide from $1989-1998$ in 
New Zealand had a current or lifetime history of contact with Child, Youth and Family (Beautrais, 2001). Third, $41 \%$ of those attending drug and alcohol services were found to have been in Child, Youth and Family care (Schroder, Sellman, Frampton, \& Deering, 2008).

In summary, while one study showed rates of lifetime and past year diagnoses in foster youth were comparable to the general population, other studies have shown comparable rates for past year diagnoses but elevated rates for lifetime diagnoses, or elevated rates for both lifetime and past year diagnoses. The most common diagnoses were conduct problems in boys and PTSD in girls. The comparable rates of past year diagnoses are attributable to rises in mental disorders in the general population at adolescence. New Zealand studies have uniformly shown elevated rates, including of completed suicide.

\section{Prevalence of mental health problems in adults formerly in foster care}

Studies of adults who have been in foster care, or who had a child welfare file in childhood, show markedly elevated rates on mental health problems. In the New Zealand Mental Health Survey, participants with a child welfare file had significantly higher rates of all past year or lifetime mental disorders, and 5 - 10 times the rate of PTSD, (Scott, et al., 2010). The results held even when those who reported retrospective childhood maltreatment were included in the comparison group. This study is thought to be the first study examining "the association between a history of prospectively ascertained child maltreatment and a wide range of later DSM-IV mental disorders" (Scott, et al., 2010, p. 712). In the Dunedin Multidisciplinary Health and Development Study, participants with a history of physical or sexual abuse, and/or two or more changes of primary caregiver, had a higher risk of depression as adults (Danese et al., 2009).

In a large, nationally representative sample in the US, about a third (30\%) of foster care alumni had a lifetime diagnosis of PTSD, which was almost 4 times the rate of the general population, and comparable to the rate for Vietnam war veterans (Pecora et al., 2005). A quarter of the alumni had PTSD in the past year, which was over 5 times the rate of the general population. Examining predictors of depression the authors concluded that optimal foster care experiences halved the rate of depression and that "... preparation for leaving care resulted in the largest reduction in the estimated probability of depression, followed by foster family and other nurturing supports while in care and educational services and experiences" (White et al., 2009, p. 44). 
In summary, rates of mental health problems are substantially higher in adults who have been in foster care compared to the general population and rates of PTSD are markedly higher than both the general population and children and adolescents who have been in foster care.

\section{Does foster care worsen behaviour problems?}

The causes of these elevated rates of mental health problems in foster care may be attributable to the adverse environments and maltreatment the children have experienced, the disruption and separation involved in entering care, especially with multiple placements, or a combination of the two (Lawrence, Carlson \& Egeland, 2006; Leathers, 2006; Leathers et al., 2012). Little attention has been paid to the reasons for the preponderance of externalising behaviour problems, especially when separation from birth families and communities would be expected to increase internalising problems (Unrau, et al., 2008). Trauma has been found to be one possible mediating factor in the relationship between maltreatment and externalising behaviour problems (Milot et al., 2010) and may be a crucial factor to investigate.

It is also important to examine why externalising behaviour problems persist despite improvements in the child's environment and whether foster care itself exacerbates, makes little difference or improves these rates of mental health problems. The extent to which exposure to early adversity, maltreatment and removal from home each contribute to these high rates of mental health problems is largely unknown but more recent studies have begun to attempt to answer these questions, which will be addressed in this section.

One possibility for the elevated rates of mental health problems in foster care is that child welfare has become a default service for children with severe mental health problems (Calvert, 2000; Lyons \& Rogers 2004). Over a quarter of children and youth who had their first psychiatric hospitalisation were placed in foster care in the next four years, compared to only $3 \%$ of other children at risk (Park, Solomon, \& Mandel, 2007). This finding suggests that the numbers of children and youth with mental health diagnoses in foster care may be skewed by those who come into care because of mental health concerns.

The onset of mental health problems may occur before, during or after foster care. Sixty per cent of the adolescents in one study reported that their mental disorder had occurred before entry into foster care (McMillen et al., 2005). Externalising problems tended to emerge before entry into foster care and internalising problems emerged after entry into care. 
Different disorders may emerge at different times. For example, Keller et al. (2010) found that of those who developed PTSD in their sample, $49 \%$ developed it before entry into care and $43 \%$ developed it after entry into care. In relation to alcohol disorder, most developed it after entry into care (69\%). These findings reflect the most common onset of substance problems. White et al. (2009) found that the quality of the foster care experiences had a large effect on rates of adult disorders, suggesting that these may also be related to experiences in foster care.

A prospective study has shown that children who enter foster care have an increase in behaviour problems shortly after entering care, and again on leaving care, and these rates were higher than those of children who received adequate care within a disadvantaged home or those who remained with a maltreating caregiver (Lawrence et al., 2006). However, children who are removed from home differ from children remaining at home after a child welfare investigation, such as having parents with higher risk (Berger, Bruch, Johnson, James, \& Rubin, 2009). Comparing unmatched samples, children who were placed in foster care had significantly more internalising and externalising problems than children who had a child welfare file. Using matched samples, however, no associations between placement and internalising or externalising behaviour problems were found. The authors conclude:

"On the whole, our results suggest that, on average, out-of-home placement appears to neither place additional burden on the already vulnerable children who enter state custody nor contribute to improved well-being for these children, at least in terms of short-term changes in cognitive skills and behaviour problems" (Berger et al., 2009, p. 1871).

Similarly, Berzin (2008) used propensity score matching and found no significant differences between young people in foster care and the matched sample on issues involved with transition to adulthood such as homelessness, drug use and teen pregnancy. The author concluded that these poor outcomes stem from pre-placement characteristics. Studies of foster children have come to similar conclusions. Foster children's emotional and behavioural problems were found to be stable over a period of five to eight years and were related to placement breakdown (Biehal, Ellison, Baker et al., 2011). The study concluded: "The severity of children's emotional and behavioural difficulties may therefore be largely determined by pre-placement adversity and the length of children's exposure to these adversities" (Biehal, Ellison, Baker, et al., 2011, p. 3).

In summary, while children's behaviour problems are unlikely to improve in foster care, studies using matched samples have not found negative effects of foster care per se. 
Elevated rates of mental health problems are likely to be a result of the child's experience of adversity and maltreatment, prior to foster care entry.

\section{Factors associated with placement disruption}

Multiple placements are common in foster care. The majority of studies have found that placement disruption is related to children's externalising behaviour problems and this was confirmed in a recent meta-analysis involving over 20,000 children, which showed that when other factors are controlled for, behaviour problems are a strong risk factor for placement breakdown (Oosterman, Schuengel, Wim Slot, Bullens, \& Doreleijers, 2007). Small to moderate associations were also found between the child's older age at placement and a history of residential care and placement breakdown. Unexpectedly, kinship care did not emerge as a protective factor against placement breakdown, but quality of caregiving and nurturing did. Similarly, adoption versus foster care was the best predictor of stability in another study, which also found kin care was not a protective factor (Proctor et al., 2011). Involvement of the adoptive or foster father, high family expressiveness, high intellectual functioning of the child and low externalising behaviour problems were also important predictors of stability.

A more recent meta-analysis examined the factors associated with placement stability and instability by reviewing 77 studies of the relationship between foster child characteristics and foster care outcomes such as permanency, reunion with birth family and re-entry into care (Pritchett, Gilberg, \& Minnis, 2013). Ninety per cent of the studies showed that children's mental health and behaviour problems had a detrimental effect on placement outcome. Three quarters of the studies showed that younger children had better outcomes, especially in achieving permanency, but only one quarter found a positive effect for older children. Foster parents themselves reported the child's characteristics affecting outcome as the special or complex needs of the child, the child being a danger to the family and the child's conduct (Pritchett et al., 2013).

Placement breakdowns in themselves are related to increases in oppositional behaviour and poor mental health, with an increasing likelihood that future placements will also disrupt (Newton, Litrownik \& Landsverk, 2000; Rubin et al., 2004). For each increase in the number of daily behaviour problems, above 6 problems in a 24 hour period, Chamberlain et al. (2006) found there was a $17 \%$ increase in the risk of a placement disruption within the next 12 months. In addition, each prior placement the child had experienced increased the hazard 
of future placement disruption by $6 \%$ (Chamberlain, Landsverk, Reid, Leve, \& Laurent, 2008). Australian studies have shown that 15 to $20 \%$ of children in care have high placement instability and this group of children is characterised by multiple abuse and neglect, a five fold increase in emotional problems and a seven fold increase in conduct problems (Barber \& Delfabbro, 2004). Placement disruptions are particularly high for adolescents with conduct problems (Barber, Delfabbro, \& Cooper, 2001). Once children start on a trajectory of placement disruption due to difficult behaviour it is likely to continue, with children who had two or more behaviour related disruptions having only 5\% chance of achieving placement stability two years later (Barber \& Delfabbro, 2004). A related study of children who had frequent placement moves because of their behaviour found that half of the children referred for new placements had had 10 or more placements and a further $12 \%$ had experienced 20 or more placements (Osborn, Delfabbro \& Barber, 2008).

In the only study to consider the neuropsychological effects of placement instability, Lewis, Dozier, Ackerman, and Sepulveda-Kozakowski (2007) found that children with unstable placements had less inhibitory control and higher oppositional behaviour than children with more stable caregiving. Multiple placements have also been found to increase rates of attentional and oppositional behaviours in foster children and incarceration in adolescents, increased severity of conduct symptoms and greater child maladjustment (Simmel, Brooks, Barth, \& Hinshaw, 2001).

In summary, placement change has been shown to be particularly detrimental for foster children, with increases in externalising behaviour leading to further instability. Some children with severe problems have little chance of achieving placement stability without intervention.

\section{Summary and conclusions}

Prevalence findings are surprisingly consistent in suggesting a rate of mental health problems in foster children of 40 to $50 \%$. Further studies need to focus on foster care itself, rather than child welfare, to disentangle the impact of foster care compared to early adversity. Studies which have compared foster children to other groups of disadvantaged children show elevated rates for children in foster care when compared to children living in poverty or disadvantaged families (e.g. Farmer, Burns, Wagner, Murray, \& Southerland, 2010; Meltzer et al., 2003). The most prevalent diagnoses for foster children are externalising behaviour problems, especially in boys, and PTSD, especially in girls. Attachment disorder and PTSD 
may be under-diagnosed (Ai, Foster, Pecora, Delaney, \& Rodriguez, 2013; Milot et al., 2010). In contrast, contradictory results have been found for foster youth, regarding both past year and lifetime diagnosis, with some studies showing marked elevations and some not finding this, suggesting further research is required. The most common diagnoses for adolescents are externalising behaviour problems, PTSD, suicidality and substance abuse. Formerly fostered adults have at least double the rate of mental health problems of the general population and up to 5 to 10 times the rate of PTSD (Scott et al., 2010).

While it is clear that foster children and adolescents, as well as adults who have been in care, have elevated rates of mental health problems and that some of these problems develop after the child has been placed in care, studies using matched samples have found that foster care does not have either a positive or harmful effect on children's behaviour problems and these are largely attributable to prior adversity. Externalising behaviour problems are related to placement breakdown, which is itself a risk factor for future placement stability.

Much foster care research ignores the complexity of the foster child's attachment and trauma histories and discrete diagnoses may not capture the full picture of foster children's mental health problems (Ai et al., 2013; Tarren-Sweeney, 2008b). The next chapter considers theoretical concepts which have been applied to foster care, or which may usefully be applied to foster care, and the impact of foster parent's psychological functioning on their foster child's outcomes. 


\section{Chapter Three-Psychological factors in foster care: Attachment, mind- mindedness and emotional availability}

Research on foster children's mental health, presented in Chapter Two, strongly supports a theoretical approach which takes into account multiple factors, which interact in complex ways, to produce change in the child's functioning. For example, it is likely that the most robust predictor of the foster child's mental health is the length of time the child spent in an adverse environment, and yet research also shows that foster children's behaviour towards new caregivers differs, and in turn changes the behaviour of new caregivers (StovallMcClough \& Dozier, 2004). Foster parent characteristics, especially the parent's state of mind about attachment and sensitivity to the child's cues, also strongly influence the child's development of secure attachment, which is related to optimal socio-emotional development (Dozier et al., 2001; Thompson, 2000).

These findings are consistent with the transactional theory of child development (Sameroff, 2009). Transactional theory represents a departure from deterministic and linear models with an emphasis on stimulus-response and nature versus nurture. Sameroff (2009) describes it as "The transactional model stresses the plastic character of the environment and of the organism as an active participant in its own growth" (p.8). Related theoretical approaches used in the study of maltreatment are ecological-transactional theory and developmental psychopathology approaches (Cicchetti \& Lynch 1993; Cicchetti \& Valentino, 2006). Cicchetti and Lynch propose that there are four levels of influence on the child including the wider culture, neighbourhood and community, the family and the individual. The cumulative risk model proposed by Rutter (1979) has been widely supported, although there is still debate about whether the effects of adverse events on children's functioning are additive or whether there is a threshold above which they have multiplying effects (Appleyard, Egeland, van Dulmen, \& Sroufe, 2005; Raviv et al., 2010).

Although most studies in foster care examine multiple interacting factors, only one study has explicitly tested the cumulative risk model in foster care (Raviv et al., 2010). This study found that a cumulative risk index predicted mental health symptoms and differentiated foster children in the clinical range on the Child Behaviour Checklist. The high incidence of risk associated with foster care, however, meant that the predictive value of variables such as maltreatment, parent substance abuse and exposure to domestic violence, was reduced compared to community samples, as over half of the foster children had these risk factors. 
Few researchers in foster care situate their study within an overarching developmental or psychological theory. Recent exceptions are studies within the ecological/transactional theory (Bell et al., 2013) and cumulative risk (Raviv et al., 2010). The first study in this thesis is based on an ecological/transactional theoretical approach and although the study is cross-sectional, a major focus is how children's previous experiences and foster parent's psychological characteristics interact to influence child outcomes. The thesis examines the theoretical constructs of a) attachment b) mental representations, in particular mindmindedness and c) emotional availability. These constructs are the focus of this chapter.

\section{Attachment theory}

Attachment denotes the way that young children organise their behaviour, and develop mental representations or ideas about themselves and other people, from the type of early experiences they have and care they receive from their parent or caregiver (Bowlby, 1969/1982). These mental representations are thought to develop into an internal working model, which the child uses to guide future behaviour (Thompson, 2000) and to become consolidated over time (Bretherton \& Munholland, 2008). This model influences how children respond to others, regardless of the way they are treated by them. Throughout the preschool period, attachment relationships influence representational models of self and others, which become more structured and organised (Toth, Maughan, Manly, Spagnola, \& Cicchetti, 2002).

The child's attachment security can be defined as the extent to which the child develops a secure and trusting relationship with their parent and is able to turn to the parent in times of emotional distress. The child's development of a secure attachment, which is related to sensitive and responsive parenting, has been found to be associated with positive social relationships, emotional regulation and mental health (Sroufe, 2000). Insecure attachment is thought to affect the child by creating negative expectations of future relationships (Thompson, 2000). The pattern of attachment most associated with risk to the child's mental health is disorganised attachment, which is associated with maltreatment (Cyr, Euser, Bakermans-Kranenburg, \& van IJzendoorn, 2010). Behaviours indicating disorganised attachment have been described as "indicators of an experience of stress and anxiety which the child cannot resolve because the parent is at the same time the source of fright and as well as the only potential haven of safety" (van IJzendoorn et al., 1999, p. 226). 
In the developmental psychopathology literature, attachment in adults is referred to as "state of mind regarding attachment" (George \& Solomon, 2008). It is measured by the coherence and organisation of an individual's account of their childhood and current relationships with attachment figures on the Adult Attachment Interview (AAI; Hesse, 2008). Responses are classified according to whether the individual's discourse about relationships with parents and caregivers best fits a secure pattern of valuing these relationships, a dismissing pattern of idealising or minimising these relationships, or a preoccupied pattern of current anger and distress about them. Discourse can also be organised and flexible or disorganised, which is defined as fearful and unresolved about past losses (Hesse, 2008; Howe, 2011).

Research has shown that the way a parent processes and resolves early relationship experiences affects their thinking and behaviour in relation to providing care (George \& Solomon, 2008). For example, parents with a balanced and coherent narrative of their early relationship experiences have detailed and rich conceptions of their child and the parent-child relationship (Huth-Bocks, Levendosky, Bogat, \& van Eye, 2004). The parent's state of mind regarding attachment has been shown to be the strongest predictor of the child's attachment security for biological children (van IJzendoorn et al., 1999). While attachment state of mind is a key variable in the investigation of parent-child relationships in foster care, one limitation of the AAI is its lengthy administration and therefore a self report measure, based on the AAI scales, was used in this study (Cassidy, Lichtenstein-Phelps, Sibrava, Thomas, \& Borkovec, 2009). This self report measure, the Perception of Adult Attachment Questionnaire is moderately correlated with the AAI and has good reliablilty and validity.

\section{Attachment research in foster care}

Young children in foster care have been found to have similar rates of secure attachment to children within biological families (Altenhofen, Clyman, Little, Baker, \& Biringen, 2013; Cole, 2005; Dozier et al, 2001; Ponciano, 2010). For example, a wellcontrolled, longitudinal study found that just over two thirds of preschoolers who were placed as infants, were securely attached at both the initial assessment and a year later (Jacobsen, Ivarsson, Wentzel-Larsen, Smith, \& Moe, 2014). In addition, a meta-analysis has shown that young children in foster care are as securely attached as children reared in their biological family, but show more disorganised attachment (van den Dries, Juffer, van IJzendoorn, \& 
Bakermans-Kraneneburg, 2009). One study found very high rates of disorganised attachment $(45 \%)$ in infants and toddlers in foster care (Bernier \& Dozier, 2003).

It is likely that the child's age at placement affects the development of a secure attachment in foster care. Children adopted over the age of 1 year show significantly less attachment security then non-adopted children (Van Den Dries et al., 2009). Infants placed under 12 months of age form stable patterns of attachment security with their caregiver within 2 months, but children aged between one and two years take somewhat longer (Dozier et al., 2001). Infants with more risk factors show decreasing levels of secure behaviour over time, suggesting that maltreatment may have subtle detrimental effects (Stovall-McClough \& Dozier, 2004). In addition, if infants manifested chaotic behaviour in the first week of foster care, they tended to develop insecure and disorganised attachment. This suggests that the behaviour of children in foster care helps shape the attachment relationship they will have with the foster mother (Bernier, Ackerman, \& Stovall-McClough, 2004). This behaviour may interfere with the parent's ability to provide the child with the stable and nurturing care necessary to help overcome the effects of previous poor care.

Foster parent's state of mind regarding attachment is strongly related to the security of attachment the child in care develops with them (Dozier et al., 2001). In other words, if the foster parent has secure attachment, the young child tends to become securely attached, at very similar rates to biological parent-child relationships (Dozier et al., 2001; van IJzendoorn, 1995). However, when children are placed with a caregiver who has an insecure state of mind regarding attachment, and who is less nurturing, they tend to show disproportionate rates of disorganised attachment, linked in turn to poor mental health outcomes (Carlson, 1998; Cole, 2005; Dozier et al., 2001). This is in contrast to the biological children of insecure parents, who tend to also develop an insecure attachment. This poses a lesser risk factor than disorganised attachment (van Ijzendoorn et al., 1999). It seems, therefore, that foster children are unable to overcome the effects of poor early care unless given optimum nurturing (Dozier et al., 2001).

Research on the attachment security of older children and adolescents who have been fostered or adopted show rates of secure attachment of $46-59 \%$, compared to studies of normative samples with 62\% with secure attachment (Barcons et al., 2014; Escobar \& Santelices, 2013; Joseph, O’Connor, Briskman, Maughan, \& Scott, 2013; van IJzendoorn et al., 1999). The first study of children aged 10 to 16 years in foster care found that just under half of the young people (48\%) had a secure relationship with their foster parents. There was no significant difference between secure attachment of children in foster care and in the 
community, but the rate of secure attachment was relatively low for both groups. Secure attachment was associated with the foster mother's positive behaviour and the duration of the placement, but not the number of previous placements or contact with birth families. The authors concluded that “...working models of attachment in adolescents who experienced severe maltreatment nonetheless remained open and responsive to day-to-day caregiving experiences" (Joseph et al., 2013, p. 10). Nevertheless, the study also found a higher rate of avoidant attachment (42\%) compared to $15 \%$ found in normative samples (van IJzendoorn et al., 1999).

Similarly, a study of adolescents adopted as infants or young children found comparable rates of secure attachment but somewhat higher rates of ambivalent and avoidant attachment than the general population (Barcons et al., 2014). Unexpectedly, these studies found very low rates of disorganised attachment $(0-12 \%)$, which contrasts with $15 \%$ of normative samples (van IJzendoorn et al., 1999) and to a meta-analysis of studies with younger children (van den Dries et al., 2009). High rates of avoidant attachment are not surprising as this develops when children are rejected by their parents, but the low rates of disorganised attachment are surprising as this is associated with maltreatment (Cyr et al., 2010).

Change in the security of attachment representations has been found to occur in foster care and adoption, but only slowly over time. Two studies have shown that foster children may change some aspects of their attachment representations of foster parents over time, such as that adults may be helpful. However, the children's views of adults as rejecting and aggressive persisted (Steele, Hodges, Kaniuk, Hillman, \& Henderson, 2003: Dallos, MorganWest, \& Denman, 2014).

In summary, a key finding is that very young foster children develop secure attachment at a similar rate to biological children. Caveats to this finding are that older toddlers are less likely to develop secure attachment, and if a foster parent is less nurturing, foster children are more likely to develop disorganised attachment. Secure attachment is related to later social and interpersonal competence, while disorganised attachment predicts higher rates of mental health problems in adolescence and adulthood (van IJzendoorn et al., 1999). Studies examining attachment security in older foster and adopted children have found, compared to normative samples, lower but comparable rates of secure attachment, higher rates of avoidant attachment, and surprisingly, low rates of disorganised attachment.

The mechanisms through which parents influence their child's attachment security are yet to be fully understood. Parent sensitivity has been found to be the main predictor of the 
child's attachment security, and yet it accounts for only a small amount of the variance in the child's attachment security (van IJzendoorn et al., 1999). This puzzling finding has been termed the "transmission gap" and has lead researchers to study other contributors to attachment security (Bernier \& Dozier, 2003). The main alternative contributor which has been investigated is parents' mental representations, which are covered in the next section.

\section{Mental Representations and Mentalisation}

Peter Fonagy and his colleagues have coined the terms mentalization and reflective function to convey the ability to make sense of oneself and other people's behaviour in interpersonal contexts (Sharp \& Fonagy, 2008). Many studies have found a link between children's mentalizing ability and their primary attachment relationship, which is mediated by "the coherence and mentalizing nature of the general discourse in the home" (Fonagy \& Bateman, 2008, p. 11). The ability of mothers to adopt a psychological perspective on their child influences the development of emotion understanding and theory of mind (ErekyStevens, 2008; Meins, Fernyhough, Arnott, Leekam, \& De Rosnay, 2013). Bouchard et al. (2008) argue that the ability to apply psychological explanations or mental models to others provides a "symbolic buffer between the behaviour of significant others and their impact on the developing mind" (p. 60). The authors also propose that maltreatment impairs a child's ability to self reflect, as he or she avoids thinking about the mental states of others, especially abusive adults (Bouchard et al., 2008). It is thought that the child's capacity to self reflect is enhanced by parents ascribing mental states to the child and treating the child as a psychological agent, and this has been linked to the security of the child's attachment (Sharp \& Fonagy, 2008).

\section{Mental Representations, Maltreatment and Foster Care}

Children who have been maltreated are likely to hold negative representations of their birth parents and corresponding negative representations of themselves (Milan \& Pinderhughes, 2000; Toth et al., 2002). This holds true even for children aged between 5 and 6 years, who had been placed in foster care prior to 19 months of age, who had been in stable placements since infancy (Ackerman \& Dozier, 2005). This is in contrast to typically developing children, who have a positive and idealised view of self at this age.

In a study of older children in foster care, the children's negative perceptions or mental representations after maltreatment by their biological mother were related to the level of maltreatment by the mother (Milan \& Pinderhughes, 2000). These negative perceptions also 
influenced their new relationship with their foster mother. Successful placements for older children are associated with highly sensitive caregivers (Schofield \& Beek, 2005a, 2005b). These caregivers had an ability to reflect and empathise with the child, believed they were central to the child's well being and had developed theories about the child's past, which helped them to explain the child's present behaviour. Eighty three per cent of the children in the care of these sensitive caregivers were making good progress three years later, compared to $50 \%$ of the children in the care of those rated less sensitive. The concept of mindmindedness is relevant to this finding in that the way the caregivers thought about the child that is, inferred the child's mental states - influenced placement outcome.

\section{Mind-mindedness theory and research}

The ability to accurately "read" the child's cues has been investigated by Elizabeth Meins and her colleagues, who have adopted the term "mind-mindedness" to refer to parents' tendency to treat their child as a separate person with their own thoughts and mental life (Meins, 1997). These researchers have shown that parents differ in the extent to which they are "mind-minded". Mind-mindedness can be measured by rating the proportion and accuracy of mind-related comments in an observation of parent-infant interaction. Accuracy is rated by an observer who notes when the child's behaviour corresponds to the parent's comment: for example, the child reaches for a shoe and the parent says "You want the shoe". Misattuned comments are rated when the child pushes the shoe away with disinterest and the parent says "You want the shoe" (Meins, 2013). After Demers, Bernier, Tarabulsy and Provost (2010 a, 2010b), mind-related comments can also be rated as positive, for example, "he is imaginative"; negative, for example, "she is very angry"; or neutral, for example, "she likes reading".

For older children, the parent is asked to describe the child and the proportion of mindrelated comments is calculated. Mind-related comments include references to the child's mind, intellect, knowledge, preferences and emotions (Meins \& Fernyhough, 2010). Mindmindedness appears to be an aspect of a personal relationship, rather than solely a stable cognitive or verbal trait (Meins, Fernyhough, \& Harris-Waller, 2014). Mind-mindedness is conceived as a precursor to parent sensitivity, that is, accurately interpreting the child's cues is necessary before one can respond sensitively (Meins, 2013).

In the general population, parents who treat their young children as if they have a mind of their own, and who use a high level of mind-related comments in conversation with them, 
or about them, have children with a range of positive social and cognitive outcomes (Meins \& Fernyhough, 1999; Meins et al., 2003). These include earlier development of theory of mind, higher executive functioning and secure attachment (Bernier, Carlson, \& Whipple, 2010; Laranjo, Bernier, \& Meins, 2008; Meins, 2013; Meins, Fernyhough, Fradley, \& Tuckey, 2001).

Meins (1997) proposed that parent's levels of mind-mindedness may help to explain how attachment states are transmitted from one generation to the next. Supporting this, studies have shown that mind-mindedness is related to both the parents' state of mind about attachment and the child's attachment security. Importantly, these relationship have been found using both the observational measure of mind-mindedness (Arnott \& Meins, 2008; Larango, Bernier, \& Meins, 2008) and the interview measure (Demers et al., 2010b; Meins, 1998; Meins, Fernyhough, Russell, \& Clark-Carter, 1998). Positive mind-mindedness is also related to mothers' state of mind regarding attachment (Demers et al., 2010b). Findings on the role of mind-mindedness have been contradictory, however. Some studies have found that mind-mindedness is an independent predictor of the child's attachment security (Meins et al., 2001; Meins et al., 2012; Meins et al., 2013). Other studies have shown that parent mindmindedness does not have an independent effect on the child's attachment security, but rather that sensitivity mediates the relationship between parent mind-mindedness and the child's attachment security (Laranjo et al., 2008; Lundy, 2003).

The relationship between parent mind-mindedness and parent sensitivity is also less than clear. Only the infant observation measure of mind-mindedness has shown a relationship with maternal sensitivity (Demers, et al., 2010a; Laranjo et al., 2008; Meins et al., 2001). When an interview measure has been used, the relationship between mindmindedness and sensitivity has been statistically non-significant (Demers et al, 2010b; McMahon \& Meins, 2012; Meins, Fernyhough, Russell, \& Clark-Carter, 1998). However, interview studies have found a relationship between positive mind-mindedness and both maternal sensitivity and another aspect of parenting, namely non-hostility (Demers et al, 2010b; Lok \& McMahon, 2006; McMahon \& Meins, 2012). For example, McMahon and Meins (2012) found that mothers with higher mind-mindedness, and higher positive mindmindedness, were less hostile towards the child, and this relationship was mediated by maternal stress. The authors argue that "even demanding behaviour on the part of the child may be experienced as more understandable and, therefore, less stressful (McMahon and Meins, 2012, p. 250). It is also possible that parents who have low stress levels and who do not perceive the child as difficult may construct “... more positive and more mind-minded 
representations of the child ..." (Demers et al., 2010b, p. 106). A longitudinal study would add clarity to this issue.

Two studies have examined whether parent mind-mindedness is related to children's emotional and behaviour problems. In one study, mothers' mind-mindedness towards their infants was related to behavioural difficulties three years later and when the children started school, but only in the low socio-economic group (Meins, Centifani, Fernyhough, \& Fishburn, 2013). Mothers in the low socio-economic group had higher levels of maternal depression and lower perceived social support, and their children had lower child language ability. Mind-mindedness was found to lessen the negative effects of these variables on children's later behaviour problems. Another study found that mind-mindedness was related to children's emotional and behaviour problems in a community sample but not a clinic referred sample (Walker, Wheatcroft, \& Camic, 2012).

Mind-mindedness may be particularly relevant to foster care. Meins (2013) argued that mothers can respond sensitively to their children because "...the mother has extensive knowledge of the child, accumulated via careful observation of what cues have meant in the past, what responses have been successful in dealing with such cues, and so on.” (p. 529). Foster parents usually have little history on which to base their interpretation of the meaning of the child's cues and successful responses to them, suggesting higher demand for mindmindedness skills in foster parenting.

In summary, mind-mindedness has been linked to a variety of positive socio-emotional outcomes for children and studies have recently begun to be examine this concept in clinical populations. Taken together, the findings of mind-mindedness research suggest that "The effects of mind-mindedness on parenting are likely to be part of a wider network of processes whereby attachment security, sensitivity and mind-mindedness interact and impact upon each other throughout the course of parenting" (Farrow \& Blissett, 2014, p. 231).

\section{Emotional availability theory and research}

Emotional availability is another concept which has been utilised to try to understand the way that parents' state of mind about attachment influences the child's attachment security. The concept of emotional availability focuses on the quality of the emotional connection and communication within the parent-child relationship (Biringen \& Robinson, 1991). It is based on Ainsworth's notion of maternal sensitivity (Ainsworth, Blehar, Waters, $\&$ Wall, 1978) but is wider in scope. Other differences include that: emotional availability is 
measured under routine, low stress conditions; it assesses a broad range of emotions; and it is a property of the particular parent-child relationship, rather than an individual construct (Biringen, 2000; Bretherton, 2000). The Emotional Availability Scale (EAS, $4^{\text {th }}$ edition; Biringen 2008) includes a measure of parental sensitivity but also measures the parent's level of structuring, non-hostility and non-intrusiveness, as well as the child's responsivity and involvement in the interaction.

Emotional availability has been linked to a variety of developmental outcomes, including sleep patterns in infancy, language acquisition in deaf children and aggressive behaviour in 4 year olds (Biringen, 2000; Biringen, Damon et al., 2005). It has been shown in several studies to be strongly related to both the security of an infant's attachment and maternal attachment representations, even when attachment is measured in infancy and emotional availability is measured in middle childhood (Aviezer, Sagi, Joela, \& Ziv, 1999; Easterbrooks, Biesecker, \& Lyons-Ruth, 2000).

The inclusion of measures of the child's contribution to the emotional aspect of the parent-child relationship make the EAS a particularly relevant measure for children in foster care. There is greater potential for the relationship between foster parents and children to be affected by the previous adverse experiences of the child (Milan \& Pinderhughes, 2000). In addition, the foster parent-child relationship is affected by the "foster mother's expectations and interpretations of a foster child's behavior, which usually includes an array of emotional deficits and behavioural problems" (Lawler, 2008, p. 1031). As young children in care may not signal their need for nurturance (Stovall \& Dozier, 2000), the EAS may be particularly useful for this population as it is a global measure of emotional exchange within a relationship, and the child's contribution can be assessed, as well as that of the parent.

Two studies have used the EAS with children in care and their foster parents (Altenhofen et al., 2013; Lawler, 2008;). Lawler's (2008) study included maltreated children, between 2 and 8 years of age, who were receiving mental health services. The study compared the emotional availability of kin and non-kin foster carers and found that, contrary to expectations, non-kin foster mothers were just as emotionally available to their foster children as were kin foster mothers (Lawler, 2008). In a sample of three year old children placed as infants, a relationship between secure attachment and three combined aspects of emotional availability, which were caregiver sensitivity, child responsivity and child involvement, was found (Altenhofen et al., 2013). Maternal emotional availability has also been studied in a group of biological and foster parents of babies exposed to drugs in utero 
but the analysis of the mixed sample makes it difficult to draw conclusions about the emotional availability of the foster parents (Swanson, Beckwith, \& Howard, 2000).

Several studies have examined the relationship between post-natal depression and chronic depression and the mother's emotional availability to the child (Cornish, McMahon, \& Ungerer, 2008; Trapolini, Ungerer, \& McMahon, 2008). One study found that chronic maternal depression was related to lower levels of maternal sensitivity. Importantly, this relationship was mediated by the mother's capacity to take the child's perspective when asked about her relationship with the child, as assessed by the Parent Development Interview (Aber, Slade, Berger, Bresgi, \& Kaplan, 1985). The authors concluded that “ ... at 4 year follow-up the caregiving representations of mothers who had been depressed...were distinguished by fewer attempts to understand their child's internal states and take their child's perspective into account" (Trapolini et al., 2008, p. 86).

In summary, parent emotional availability has been found to be related to aspects of children's socio-emotional development and, in one study, to foster children's attachment security. The inclusion of scales rating the child's emotional availability to the parent makes this concept particularly applicable to foster care.

\section{Parent motivation and commitment}

Other aspects of foster parent psychological functioning have been investigated in relation to foster care. Two of these are foster parents' motivations for providing care and foster parents' acceptance of and commitment to the child. Considering the first of these, foster parents' motivations or reasons for providing foster care for infants have been found to affect the subsequent security of the infant's attachment (Cole, 2005). Reasons such as wanting to increase family size, wanting to make a difference for children and rescuing children from abuse have been found to increase secure attachment. Religious reasons, replacing an adult child and fostering with a view to adopting the child decreased rates of secure attachment.

Significant relationships have been found between parents' higher commitment to the child and longer length of placement, higher child self esteem and decreases in behaviour problems and levels of aggression (Ackerman \& Dozier, 2005; Lindhiem \& Dozier,2007). The caregiver's acceptance of the child at age two years was related to the child's self perceptions at age 5 or 6 (Ackerman \& Dozier, 2005). The authors concluded that children who have had early disruptions of care may develop maladaptive self perceptions and coping 
strategies when placed with caregivers who are not fully emotionally invested in their foster children. A further study by the same group found that foster parents' observed "delight" in their child was related to their commitment to the child (Bick, Dozier, Bernard, Grasso, \& Simons, 2013).

\section{Summary}

There is evidence that parent sensitivity and state of mind about attachment are important contributors to both biological and foster children's wellbeing. Mental representations, and in particular mind-mindedness, have been shown to be influential in children's socio-emotional functioning, but have not been studied in foster care. There are a number of reasons why parent mental representations may be even more important for foster children than children in the community. Chief among these is the difficulty in interpreting children's cues, when the foster parent does not have a shared history with the child. The current study will examine these concepts, along with foster parents' motivations for providing care, their acceptance and commitment to the child and the child's demographic and placement characteristics. This study is described in the following chapter. 


\section{Chapter Four-Study 1: The relationship between foster child characteristics, foster parent psychological functioning, and two child outcomes}

\section{Introduction}

\section{Challenges and barriers to sensitive foster parenting}

A key competency in the provision of foster care is the ability of foster parents to build a supportive and nurturing relationship with the child in their care (Buehler, Rhodes, Orme, \& Cuddeback, 2006). Positive foster parent-child relationships are crucial to the child's recovery from maltreatment, as placement disruptions due to foster parents being unable to manage the child's difficult behaviour have been linked with increased child behaviour problems and a decreased chance of a subsequent successful placement (Barber \& Delfabbro, 2004).

There are several barriers to successfully developing a positive relationship from the point of view of both the child and the parent. Child barriers include showing resistant and unpredictable behaviour towards their new caregiver (Stovall-McClough \& Dozier, 2004). Parent barriers include difficulties in managing complex behaviour problems, which may relate to prior attachment disruptions and trauma (Murray et al., 2011). A potentially important foster parent factor, which will be investigated in this study, is the difficulty of interpreting and understanding the child's mental state, behaviour and experience. Without an accurate interpretation and understanding of the child's behavioural communication, optimal parenting is unlikely.

Most children in foster care have grown up in environments characterised by high levels of interpersonal conflict and negative emotion, in which they have experienced abuse or neglect from parents who often have mental health and substance abuse problems (Osborn, et al., 2008). As a consequence, children frequently experience difficulties with emotional regulation and associated behavioural problems (Shipman et al., 2007). They may also have negative expectations of future care by foster parents (Milan \& Pinderhughes, 2000). These difficulties place many demands on foster parents and increase the risk of placement breakdown and a further escalation of problem behaviours. Particularly problematic, many of these children do not receive any specialist mental health care (Burns et al., 2004). This places further demands on foster parents to manage the child's emotional and behavioural difficulties without specialist support. 
In summary, the challenges foster parents face - along with their lack of history with the child - suggest higher cognitive demands may be made on their ability to reflect on and interpret the child's behaviour, as well as provide sensitive care. Both of these factors have been found to be associated with successful placements.

\section{Aims of the study}

The current study aims to address a gap in the research literature by examining foster parent mental representations, conceptualised here as mind-mindedness, in addition to other aspects of foster parent psychological functioning. After Demers et al. (2010a, 2010b) the valence of mind-mindedness were also examined, that is, positive mind-mindedness (the proportion of positive mind-related comments), and negative mind-mindedness (the proportion of negative mind-related comments). The studies on which the current hypotheses are based are described in Chapter Three.

The first set of hypotheses concerned foster parent mind-mindedness. Specifically, it was predicted that foster parent mind-mindedness would be negatively related to foster children's emotional and behaviour problems and positively related to the quality of the parent-child relationship. It was also predicted that positive mind-mindedness would have inverse associations with foster children's emotional and behaviour problems and positive associations with the parent-child relationship. The converse was expected for negative mind-mindedness.

The second set of hypotheses concerned aspects of foster parent psychological functioning found to be related to foster children's socio-emotional development in at least one study. Specifically, it was predicted that foster parents' positive self-reported attachment perceptions, emotional availability and commitment to the foster child would be inversely associated with the child's emotional and behaviour problems and positively associated with the quality of the parent-child relationship. It was also hypothesised that these foster parent psychological variables, along with foster parent, depression, anxiety and stress, may mediate or moderate any relationship between mind-mindedness and the outcome variables. Another aspect of foster parent psychological functioning is their motivation for providing foster care. The relationship between foster parent motivation and the child's outcome variables was explored, but no specific hypothesis was made, due to limited research on this topic.

The final set of hypotheses concerned the relationship between the child's demographic and placement characteristics and the outcome variables. Specifically, it was predicted that the child's age, the age at which the child was placed, short term placements, the number of 
previous placements, and types of maltreatment the child had experienced would be positively associated with the child's emotional and behaviour problems. It was also predicted that duration of placement would not be related to the child's emotional and behaviour problems. Additionally, it was expected that the child's age and the age at which they were placed would be negatively associated with the quality of the parent-child relationship and the duration of the placement would be positively associated with it. The relationship between the child's ethnicity and the outcome variables was also examined, but no specific hypotheses were made.

\section{Method}

Participants. The participants were 62 foster parents. The parent who was considered the primary parent, that is, the parent who spent the most time with the child, participated in the research. The child who was the focus of the research was a foster child who was between 2 and 13 years of age. If the parent had more than one foster child in their care in this age range, the particular child to participate in the intervention was chosen in order, that is, the first participant reported on their oldest child in this age range, the next participant reported on the middle child, the next on the youngest and so on. Further criteria were that the child had been placed in care with the current foster family for at least 2 months and was expected to stay in the placement for at least a further 2 months. A further inclusion criterion was that English was the language used by the child and foster parent and the exclusion criterion was severe intellectual disability.

Participant characteristics. Table 1 presents the means and standard deviation values for the demographic and placement variables of the sample of children in foster care. The children had been with their current caregiver for an average of 3 years, a third of the children had been in the placement for over 3 years and one in seven had been in their placement for over 5 years. Twenty three per cent of the children were in their first placement, $25 \%$ in their second and $20 \%$ in their third placement, indicating that around two thirds of the foster children (68\%) had had two or fewer previous placements.

Foster parents were between 31 and 66 years of age and had fostered between one and over 130 children; $28 \%$ had never fostered before and $15 \%$ had fostered over 50 children. 
Table 1 Foster Parents' and Children's Demographic and Placement Variables

\begin{tabular}{|c|c|c|c|c|}
\hline & Variable & $N=62$ & Mean $(S D)$ & Range \\
\hline \multirow[t]{6}{*}{ Foster child } & $\begin{array}{c}\text { Gender: Female } \\
\text { Male }\end{array}$ & $\begin{array}{l}36(58 \%) \\
26(42 \%)\end{array}$ & & \\
\hline & $\begin{array}{c}\text { Ethnicity: NZ European } \\
\text { Other European } \\
\text { Māori } \\
\text { Other } \\
\end{array}$ & $\begin{array}{cc}30 & (48.4 \%) \\
1 & (1.65 \%) \\
30 & (48.4 \%) \\
1 & (1.6 \%) \\
\end{array}$ & & \\
\hline & Age (years) & & $7.88(3.36)$ & $2-13$ years \\
\hline & $\begin{array}{l}\text { Age at placement with current } \\
\text { caregiver (years) }\end{array}$ & & $4.96(3.70)$ & $\begin{array}{l}1 \text { month }-13 \\
\text { years }\end{array}$ \\
\hline & Length of placement (months) & & $36(34.60)$ & $2.5-154$ \\
\hline & $\begin{array}{l}\text { Number of previous } \\
\text { placements }\end{array}$ & & 3.05 & $1-25$ \\
\hline \multirow[t]{3}{*}{ Foster parent } & $\begin{array}{c}\text { Gender: Female } \\
\text { Male }\end{array}$ & $\begin{aligned} 56(90.3 \%) \\
6 \quad(9.7 \%)\end{aligned}$ & & \\
\hline & $\begin{array}{c}\text { Ethnicity: NZ European } \\
\text { Other European } \\
\text { Māori } \\
\text { Other } \\
\end{array}$ & $\begin{aligned} & 48(77.4 \%) \\
& 9(14.5 \%) \\
& 5(8.1 \%) \\
& 0 \\
&\end{aligned}$ & & \\
\hline & Age & & $47.56(8.56)$ & $31-66$ years \\
\hline
\end{tabular}

Recruitment. The participants were recruited from providers of foster care: Child, Youth and Family (CYF), New Zealand's welfare agency for children and young people, which is a service of the Ministry of Social Development, Barnardos and the Open Home Foundation, which are non-governmental providers of foster care, and from the NZ Family and Foster Care Federation (now known as Fostering Kids). Fostering Kids is a support, advocacy and education provider for foster parents and foster care professionals.

Each of these agencies gave permission for their foster parents or caregivers to be invited to participate in the studies and CYF staff assisted with setting up the study. For each participant, information sheets were emailed to the CYF local Site Manager, the foster child's social worker and the foster parent's social worker. Agreement was sought and obtained for the researcher to proceed with each foster parent who had agreed to participate, and consent was obtained from the foster parent. When children participated in the videotaped interaction, consent was obtained from birth parents. Assent was obtained from the child as appropriate to their age (eight children). Ethics approval was given by the Victoria University School of Psychology Ethics Committee, under the delegated authority of the Victoria University Human Ethics Committee, and the Ministry of Social Development Research Access Committee.

Due to recruitment difficulties, it was not possible to recruit a random sample. The sample had an under representation of Māori ethnicity (8.1\%), compared to $14.6 \%$ of the 
New Zealand population (Statistics New Zealand, 2006). However, numbers of Māori providing foster care through official channels is unknown. The sample also has an under representation of kin or family/whānau foster parents (12.9\%), compared to the $48 \%$ providing foster care.

\section{Measures}

Interview. An interview with the foster parent was conducted to cover demographic questions about themselves and the foster child, the Mind-mindedness Interview and the "This is my Baby [Child] Interview".

Demographic questions. Questions for foster parents included their age, education level, marital status and ethnicity; number of past and present foster children; number of birth children living at home or left home; whether the care is kin or non kin and participation in training (see Appendix A). Questions about the child included age at placement, length of time in placement, number of previous placements, number of types of maltreatment and contact with birth parents or family (see Appendix A).

Mind-mindedness interview. Following Meins et al. (2001), foster parent were asked one question "Can you describe [child] to me?" Following Bernier and Dozier (2003), parents were also asked "What is he or she like?" In relation to reliability, mind-mindedness measures have been shown to be stable over several months and inter-rater reliability is consistently high (Meins \& Fernyhough, 1999; Meins et al., 2003). In relation to construct validity, mind-mindedness has shown to be related to parental sensitivity, non-hostility and parenting stress (Lok \& McMahon, 2006; McMahon \& Meins, 2012; Meins et al., 1998).

Coding. The interview was audio-recorded and transcribed verbatim by a research assistant. The interview was coded according to criteria set out in the Mind-mindedness coding manual, version 2.0 (Meins \& Fernyhough, 2010). Mind-related comments include references to the child's mind, intellect, knowledge, desires, preferences and emotions, as a proportion of overall comments. Comments were coded into four mutually exclusive categories: general, physical, behavioural, and mental. Mental comments include "She's very intellectual" and "He is sad"; physical comments include "She is tall" and "He is cute"; general comments include "She is bubbly" and "He has a good personality" and behavioural comments include "She is always on the go" and "He throws tantrums". Following Demers et al. (2010a, 2010b), the valence of the mental comments was also coded as the proportion of mental comments that were a) positive, such as "He's very imaginative", b) negative, such as "She's very strong-willed" or c) neutral, such as "He likes books". The author coded all 
of the interviews and 30\% were independently coded by the research assistant as a reliability check. The kappa for mind-mindedness in this study was $\kappa=0.90$ and the kappa for the valence of mind-mindedness was $\kappa=0.94$.

This is my Baby [Child] Interview. (TIMB; Bates \& Dozier, 1998). Foster parents were asked eight questions in this semi-structured interview which measures three constructs: foster parent commitment, acceptance of the child and belief in their own influence with regard to their foster child (see Appendix A). The first question of the interview is the same as the Mind-mindedness Interview. Following Lindhiem and Dozier (2006), only the foster parent commitment scale was used in this research. In relation to reliability and validity, the TIMB interview has high inter-rater reliability, a test re-test reliability of .61 over 11 months has been reported, and good predictive validity has been shown with an association to length of placement, level of behaviour problems and emotional regulation (Bates \& Dozier, 1998; Dozier \& Lindhiem, 2006; Lindhiem \& Dozier, 2007).

Coding. The interview was audio-recorded for transcription and coded using a 5 point Likert scale, according to criteria set out in the "This is my Baby Interview" Manual (Bates \& Dozier, 2005). To code for foster parent commitment to the child, the coder simultaneously reads the transcript and listens to the audiotape, and then choses a score. A low score is assigned when the parent does not show any emotional investment in the child, a moderate score is given when the parent shows some evidence of emotional investment in the child "but the child has not been psychologically adopted" (Bates \& Dozier, 2005, p. 10) by the parent and a high score is assigned if the parent gives evidence of a strong emotional investment and a "strong attachment to the child" (p. 10). All of the interviews were coded by the author and $30 \%$ were independently coded by a research assistant. The intra-class reliability coefficient was $r=.83$.

Motivations for Foster Parenting Inventory (Yates, Lekies, Stockdale, \& Crase, 1997). This 10 item inventory uses a 5 point Likert Scale for foster parents to rate how important a range of motivations were to their decision to provide foster care and it has been used with a variety of populations (Cole, 2005; Gillis-Arnold, Crase, Stockdale, \& Shelley, 1998). The 10 motivations include financial reasons, rescuing children from abuse, and helping special needs children. Motivations such as wanting to increase family size and concern for the community have been found to be associated with attachment security in infants and reasons such as replace a child who has left home and religious reasons have been found to be associated with insecure attachment in infants (Cole 2005). Significant 
differences have also been found in parents wanting to adopt a child versus those wanting to provide foster care (Gillis-Arnold et al., 1998).

Perceptions of Adult Attachment. (PAAQ; Lichtenstein \& Cassidy, 1991). This self report measure assesses adults' perceptions of the quality of their early and current relationship with their primary caregiver, usually their mother, and their current state of mind regarding attachment. There are three subscales relating to the adults' experiences of their primary caregiver in childhood: Loving, Rejecting or Role Reversing. There are also five current state of mind subscales: Vulnerable, Balanced or forgiving, Anger, Derogation and Lack of recall of childhood memories. Examples of questions include "In childhood, I felt like I was really treasured by my mother" (Loving scale), "Even with all our past difficulties, I realise my mother did the best for me that she could" (Balanced/forgiving scale), and "My family was not particularly intimate, but this has never bothered me" (Dismissing/derogating scale). In the current study, a composite variable was created from the five current state of mind scales. The score for each scale was converted to a z score and the scores were summed. This composite variable was used in all further analyses.

Across samples of college students, adults and mothers, the PAAQ has been found to have high internal consistency, construct validity and test re-test reliability (Cassidy et al., 2009; Cicchetti, Rogosch, \& Toth, et al., 2006; Huth-Bocks et al., 2004). The PAAQ has been shown to be moderately correlated with the Adult Attachment Interview (AAI) in 4 out of 6 sub-scales (Cassidy et al., 2009). Chronbach's alphas in the current study for the childhood experience scales were Rejection $\alpha=.85$, Loving $\alpha=.87$ and Role Reversed $\alpha=$ .63. Alphas for the current state of mind scales were Vulnerable $\alpha=.75$, Balanced or forgiving $\alpha=.72$, Angry $\alpha=.74$, Derogation or devaluing $\alpha=.51$, and Lack of recall of childhood memories $\alpha=.89$.

Depression, Anxiety and Stress Scale. (DASS; Lovibond \& Lovibond, 1995). The DASS is a set of three self-report scales designed to measure the negative emotional states of depression, anxiety and stress. In a general population study, the DASS 21 had good internal consistencies ( $\alpha=.82-.93)$ and good discriminant and convergent validity, when compared to other accepted measures of anxiety and depression (Henry \& Crawford, 2005). The DASS 21 has been shown to reliably measure changes in an individual's symptoms over time (Ronk, Korman, Hooke, \& Page, 2013). Chronbach's alphas in the current study were: Depression $\alpha=.76$; Anxiety $\alpha=.69$, and Stress $\alpha=.86$. 
Strengths and Difficulties Questionnaire (SDQ; Goodman \& Scott, 1999). This 25 item questionnaire has five scales which are conduct problems, inattention-hyperactivity, emotional symptoms, peer problems and pro-social behaviour. Across samples, this measure has excellent reliability and validity, with test re-test reliability over two weeks found to be .71 (Mellor, 2004), and children who had a specific diagnosis were identified with a specificity of $95 \%$ and a sensitivity of $63 \%$ (Goodman, Ford, Simmons, Gatward, \& Meltzer, 2003). Internal consistency in a nationally representative US sample ranged from fair for the Peer Scale $(\alpha=0.46)$ to excellent for the Total score $(\alpha=0.83$; Bourdon, Goodman, Rae, Simpson, \& Koretz, 2005). Chronbach's alphas in the current study were: Emotion $\alpha=.73$, Conduct $\alpha=.83$, Attention $\alpha=.79$, Peer $\alpha=.66$, and Prosocial $\alpha=.62$.

Child-Parent Relationship Scale. (CPRS, Pianta 1994). This scale is completed by a parent and contains fifteen 5 point Likert scales designed to assess the adult's view of their relationship with the child. There are two subscales - the Closeness scale and the Conflictual scale. The scales have been used in several large scale studies and internal consistency was found to be moderate with good predictive validity with measures of emotion regulation and attachment security (Howes \& Ritchie, 1999; NICHD Early Child Care Research Network, 2004). In the current study, Chronbach's alphas were Closeness $\alpha=.81$ and Conflict $\alpha=.82$.

Emotional Availability Scale. (EAS $4^{\text {th }}$ edition; Biringen, 2008). In this videotaped interaction, the parent was asked to set up the video camera and play with the child for 20 minutes, "as you normally would". This observational scale is designed for parents and children aged from infancy to 14 years, with an infancy/early childhood and middle childhood version. The EAS had acceptable inter-rater reliability and internal consistency (Lawler, 2008). Concurrent validity has been established by the associations of the various dimensions of the EAS to security of infant and child attachment, the mother's state of mind regarding attachment, mother's depressive symptomatology and levels of aggression in preschoolers (Biringen et al., 2005; Easterbrooks, Chaudhuri, \& Gestsdottir, 2005).

Coding. Each videotape was coded by two independent raters, one of whom was the author. The coders were trained by Zeynep Biringen, the developer of the EAS ( $4^{\text {th }}$ ed.), and successfully completed the reliability test. The scale rates four dimensions for parental behaviour: sensitivity, non-hostility, non-intrusiveness and structuring. The two child dimensions are responsiveness and involvement. Intra-class correlations for the current study were: Sensitivity $r=.80$, Structuring $r=.81$, Non-Hostility $r=.63$, Non-Intrusiveness $r=$ .69 , Child Responsiveness $r=.71$, and Child Involvement $r=.90$. 


\section{Procedure}

After consent procedures were completed with the foster parent, CYF and the birth parent, if applicable, foster parents took part in an interview consisting of a brief demographic interview and two semi-structured interviews: the Mind-mindedness Interview (Meins, 1998) and the "This is my Baby [Child] Interview" (Bates \& Dozier, 1998). The interview was conducted in person for the first five participants and then over the phone for the remaining participants, due to their geographical spread. The foster parents then completed five self report questionnaires. If birth parent consent was given, the foster parent was asked to play with their child for 20 minutes and this was videotaped and scored using the EAS ( $4^{\text {th }}$ edition; Biringen, 2008). Three videotapes were made in person by the researcher and the remainder were made by the foster parent themselves, with a camera and instructions sent by the researcher, again due to geographical spread.

\section{Results}

\section{Data analysis plan}

Results were obtained from four main steps of analysis. First, descriptive statistics were calculated. Second, bivariate correlations examined the relationships between predictor variables and covariates, and the outcome variables (emotional and behaviour problems and the quality of the parent-child relationship) and were used to determine which variables would be retained for further analysis. Third, hierarchical regression examined which variables were the strongest predictors of the child's emotional and behaviour problems and the quality of the foster parent-child relationship, entered separately as dependent variables. Hierarchical regression and moderation analyses were also used to test for mediating and moderating variables, using Medgraph and Modgraph (Jose, 2013a, 2013b). Finally, path analyses tested for the best predictors of the child's behaviour problems and the quality of the foster parent-child relationship, considered together, using SPSS Amos 20.

\section{Descriptive statistics and preliminary analyses}

All variables were normally distributed except for duration of placement, number of previous placements, number of types of abuse, parent depression, parent anxiety and negative mind-mindedness. These variables were all positively skewed (skewness $>2.58, p$ $<.01$, kurtosis $>2.58, p<.01)$. To reduce skew, these variables were log transformed for all further analyses, which improved the normality of the distributions. As there were no 
statistically significant relationships between the Motivations for Foster Parenting Inventory and either outcome measure $(r \mathrm{~s}>.05)$ this measure were dropped from further analysis. The experience scales of the PAAQ (Loving, Rejecting and Role Reversed) were also not significantly related to either outcome measure, and were dropped from further analysis.

Table 2 shows the mean, standard deviations, range and clinical cut-offs, where available, for each measure used. Forty three per cent of the sample scored within the clinical range on the Strengths and Difficulties questionnaire, compared to $10 \%$ expected in the general population normative samples (Achenbach, Becker, Dopfner, Steinhausen, \& Rothenberger, 2008). On the subscales of the SDQ, foster parents reported that $29 \%$ of the foster children scored in the clinical range for emotional problems, $48 \%$ were in the clinical range for conduct problems, $33 \%$ for attention problems and $41 \%$ for problems with peers. About a third of the sample (34\%) had no subscales in the clinical range, $21 \%$ of the children had one elevation, $18 \%$ had two elevations, $18 \%$ had three elevations and $8 \%$ had elevations on all four subscales. Ten per cent of the foster parents scored in the clinical range for depression, $10 \%$ for anxiety and $20 \%$ for stress.

Table 2 Means, Standard Deviations, Range and Clinical Cut-offs for Measures, where available

\begin{tabular}{lllc}
\hline Measure & Mean $(S D)$ & Range & Clinical cut-off \\
\hline SDQ Total & $15.36(8.01)$ & $1-34$ & 16 \\
DASS Depression & $3.23(4.36)$ & $0-16$ & 10 \\
DASS Anxiety & $1.90(3.18)$ & $0-14$ & 8 \\
DASS Stress & $8.40(6.84)$ & $0-28$ & 15 \\
Mind-mindedness & $0.39(0.14)$ & $0.15-0.74$ & \\
Positive MM & $0.64(0.27)$ & $0-1$ & \\
Negative MM & $0.22(0.22)$ & $0-1$ & \\
\hline
\end{tabular}

SDQ = Strengths and Difficulties Questionnaire; DASS = Depression, Anxiety and Stress Scale; MM $=$ Mind-mindedness.

\section{Zero - Order Correlations between Predictor Variables}

Relations among the main predictor variables were analysed using Pearson's correlations. As expected, children's placement characteristics were not independent. For example, the child's age at placement was positively associated with the number of previous placements the child had experienced and inversely associated with the expected length of the current placement (see Table 3). This indicates that children placed at an older age had more previous placements and were more likely to be in a short term placement. Conversely, the 
duration of placement was negatively correlated with the number of previous placements and positively correlated with the expected length of placement, indicating that children who had been in placements longer had fewer previous placements and were expected to be in a long term placement.

There were also inter-relationships between some parent psychological variables and child placement characteristics. For example, a significant positive correlation was found between parent mind-mindedness, positive mind-mindedness and the expected length of placement (see Table 3). This indicates that foster parents made more mind related comments, and more positive mind-related comments, about children whose placement was expected to be long term. Foster parents' commitment to the child was negatively correlated with the age at which the child was placed and was positively correlated with the expected length of placement and the duration of placement. This indicates that parents were more committed to children placed at a younger age, who had been with them longer, and who had a long term placement. It should be noted that the number of children parents had previously fostered was unrelated to either outcome variable.

Associations between parent emotional availability and child variables are shown in Appendix A as the small sample $(\mathrm{N}=21)$ limits the analyses which can be used and conclusions which can be drawn. Of note, however, is that a number of child demographic and placement variables were significantly associated with dimensions of both foster parent and foster child emotional availability. In particular, parent sensitivity and non-intrusiveness were negatively correlated with age placed, indicating that parents were more sensitive and less intrusive with children placed at an earlier age. 
Table 3 Correlations amongst measures

\begin{tabular}{|c|c|c|c|c|c|c|c|c|c|c|c|c|c|c|c|}
\hline & 1. & 2. & 3. & 4. & 5. & 6. & 7. & 8. & 9. & 10. & 11. & 12. & 13. & 14. & 15 \\
\hline 1. Age & & & & & & & & & & & & & & & \\
\hline 2. Placement length & $.29 *$ & & & & & & & & & & & & & & \\
\hline 3. Age placed & $.67 * * *$ & $-.50 * * *$ & & & & & & & & & & & & & \\
\hline 4. Number of placements & $.27 *$ & $-.24 *$ & $.45 * * *$ & & & & & & & & & & & & \\
\hline 5. Types of abuse & .12 & -.13 & .22 & $.24 *$ & & & & & & & & & & & \\
\hline 6. Short term placement & -.07 & $.30 *$ & $-.33 * *$ & -.22 & $-.31 *$ & & & & & & & & & & \\
\hline 8. Positive MM & $-.25^{*}$ & .001 & -.24 & -.19 & $-.32 *$ & $.37 * *$ & .11 & & & & & & & & \\
\hline 9. Negative MM & .21 & .12 & .08 & .09 & $.34 *$ & -.20 & .02 & $-.72 * * *$ & & & & & & & \\
\hline 10. PAAQ & -.17 & -.17 & -.03 & -.009 & -.01 & -.03 & .003 & .23 & $-.27^{*}$ & & & & & & \\
\hline 11. DASS Depression & -.03 & .03 & -.005 & $-.31^{*}$ & .08 & -.07 & $-.27 *$ & -.10 & .13 & $-.35 * *$ & & & & & \\
\hline 12. DASS Anxiety & .23 & .02 & .18 & .04 & .19 & -.08 & -.19 & $-.27 *$ & $.43 * *$ & $-.40 * *$ & $.53 * * *$ & & & & \\
\hline 16. CPRS & $-.33^{*}$ & .15 & $.41 * *$ & $-.29 *$ & -.17 & $.31 *$ & .19 & $.32 *$ & $-.37 * *$ & $.37 * *$ & -.24 & $-.40 * *$ & -.17 & $.33^{*}$ & $-.71 * * *$ \\
\hline
\end{tabular}

Note. $* p<.05 ; * * p<.01$, two tailed sinificance. Due to participants not completing some questionnaires: $N=62$ for variables $1-9, N=60$ for variables 11 -13 and $N=61$ for variables 15 and 16. SDQ = Strengths and Difficulties Questionnaire; CPRS = Child-Parent Relationship Scale; MM = Mindmindedness; PAAQ = Perceptions of Adult Attachment Questionnaire. 


\section{Zero-Order correlations between covariates and outcome and predictor variables}

Standard covariates were identified including the child's age, gender, parent's education level and depression (McMahon \& Meins, 2012). In addition, due to the proportion of foster children in the sample who were Māori (48\%), ethnicity was also identified as a covariate. The child's ethnicity was not correlated with either outcome variable. The child's gender was not significantly correlated with either outcome variables (emotional and behaviour problems and the quality of the parent-child relationship). There was a significant negative correlation between the child's age and the quality of the parentchild relationship. This indicates that parents reported a better relationship with younger children. Maternal education and depression were not related to any outcome variables.

Regarding predictor variables, the child's age was negatively correlated with parent mind-mindedness, and positive mind-mindedness, indicating that foster parents made more mind related comments about younger children.

\section{Predictor variable relations with children's emotional and behaviour problems}

Parent variables. Pearson's correlations were used to test the hypothesis that foster parent mind-mindedness would be associated with children's emotional and behaviour problems. There was a significant negative correlation between foster parents' mindmindedness, positive mind-mindedness and the child's emotional and behaviour problems. This indicates that parents who made more mind-related comments and more positive mindrelated comments had children with fewer emotional and behaviour problems. In addition, children's emotional and behaviour problems were positively correlated with the parents' negative mind-mindedness, indicating that parents who made more negative mind-related comments had children with more emotional and behaviour problems.

Pearsons correlations then examined the hypothesis that foster parent attachment perceptions, emotional avilability, and parent commitment to the child would be associated with the quality of the foster parent-child relationship. Contrary to predictions, there were non-significant correlations between foster parent attachment perceptions and emotional availability and the child's emotional and behaviour problems. As predicted, however, parent commitment to the child was negatively correlated with the child's emotional and behavioural problems, indicating that foster parents were more committed to children with fewer behaviour problems. 
Regarding parent variables as potential mediators and moderators of the relationship between parent mind-mindedness and the child's emotional and behaviour problems, foster parents' stress and anxiety, but not depression, were positively correlated with the child's emotional and behaviour problems, indicating that foster parents with higher stress and anxiety had children with more behaviour problems.

Child placement characteristics. Pearson's correlations were also used to explore the hypothesis that the child's placement characteristics would be related to the child's emotional and behaviour problems. As predicted, significant positive correlations were found between the number of previous placements, the number of types of maltreatment the child had experienced, and children's emotional and behaviour problems. This indicates that children with more behaviour problems had previously experienced more placement changes and more types of abuse. Significant correlations were also found between short term placements and children's emotional and behavioural problems, indicating that children in short term placements had more behaviour problems.

\section{Predictor variable relations with the quality of the parent child relationship}

Parent variables. Regarding the hypothesis that foster parent mind-mindedness would be related to the quality of the parent-child relationship, contrary to predictions, the correlation between foster parent mind-mindedness and the foster parent-child relationship was not significant. There was a positive correlation, however, between positive mindmindedness and the quality of the parent-child relationship. Additionally, there was an inverse correlation between negative mind-mindedness and the parent-child relationship. This indicates that parents made more positive mind-related comments and fewer negative mind related comments about children with whom they reported having a better relationship.

Pearson's correlations then examined the hypothesis that foster parents with positive attachment perceptions, more emotional availability, and higher commitment to the child would report a higher quality of parent child relationship. As predicted, there was a significant positive correlation between both foster parent attachment perceptions and comittment to the child and the parent-child relationship. Contrary to predictions, foster parents' emotional availability was not significantly correlated with the foster parent-child relationship. However, there was a positive correlation between the child's involvement with the parent on the EAS and the quality of the parent child relationship (see Appendix B). 
Regarding parent variables as potential mediators and moderators, parent anxiety was negatively correlated with the quality of the parent-child relationship, indicating that parents with more anxiety reported a poorer quality parent-child relationship.

Child placement characteristics. Pearson's correlations were also used to explore the hypothesis that the child's placement characteristics would be related the quality of the parent-child relationship. As expected, the age at which the child was placed and the number of previous placements the child had experienced were negatively correlated with the quality of the foster parent-child relationship. This indicates that parents reported better relationships with children who were placed at a younger age, and who had fewer previous placements. Long term placements were also positively correlated with the quality of the parent-child relationship.

Hierarchical regression. In order to determine which combination of variables best predicted the child's behaviour problems and the quality of the foster parent-child relationship, hierarchical regression analyses were conducted. Total emotional and behaviour problems and quality of relationship scores were entered separately as dependent variables (see Table 4).

Table 4 displays the results of a hierarchical regression analysis examining the contribution of the number of previous placements, mind-mindedness and attachment perceptions to the child's emotional and behaviour problems. The number of previous placements was entered into the first block of the regression equation, foster parent mindmindedness was entered in the second block and, lastly, parent attachment perceptions were entered in the third block. Table 4 shows that the number of placements the child had experienced accounts for a unique $9 \%$ of the variance in foster children's emotional and behaviour problems, mind-mindedness accounted for a unique $7 \%$ of the variance, and parent attachment perceptions accounts for $6 \%$ of the variance in foster children's emotional and behaviour problems. The three variables accounted for $22 \%$ of the variance in behaviour problems (see Table 4). In relation to the foster parent-child relationship, Table 5 shows that the age at which the child was placed accounts for a unique $19 \%$ of the variance in the quality of the foster parent-child relationshi, mind-mindedness did not account for a significant portion of variance and parent attachment perceptions accounts for $12 \%$ of the variance in foster children's emotional and behaviour problems. The three variables accounted for $32 \%$ of the variance in the quality of the foster parent-child relationship (see Table 4). 
Table 4 Hierarchical Regression Model Predicting Quality of the Foster Parent-child Relationship and Foster Child Emotional and Behavioural Problems

\begin{tabular}{lccc}
\hline Variable & $\mathbf{R}^{2}$ change & F for $\mathbf{R}^{2}$ change & $\boldsymbol{\beta}$ \\
\hline Behaviour problems & & & \\
\hline Step 1 & .09 & 5.57 & $.31^{*}$ \\
$\begin{array}{l}\text { Previous placements } \\
\text { Step 2 }\end{array}$ & .07 & 4.49 & $-.26^{*}$ \\
$\begin{array}{l}\text { Mind-mindedness } \\
\text { Step 3 }\end{array}$ & & 4.38 & $-.25^{*}$ \\
Parent attachment perceptions & .06 & & \\
\hline Quality of relationship & & & \\
\hline $\begin{array}{l}\text { Step 1 } \\
\text { Age placed }\end{array}$ & .19 & 13.91 & $-.42^{* * *}$ \\
$\begin{array}{l}\text { Step 2 } \\
\text { Mind-mindedness }\end{array}$ & .01 & .47 & .08 \\
$\begin{array}{l}\text { Step 3 } \\
\text { Parent attachment perceptions }\end{array}$ & .12 & 10.27 & $.35^{* *}$ \\
\hline
\end{tabular}

Note. $* p<.05 ; * * p<.01 ; * * * p<.001$

Mediation. To test the hypothesis that several foster parent variables may mediate the relationship between mind-mindedness and the child's outcome variables, focused follow-up analyses were conducted to examine proposed mediators. Variables which were significantly correlated with each outcome variable were used in the regression analysis. Using hierarchical regression analyses and Medgraph (Jose, 2013), we examined whether parent depression, anxiety and stress, and parent commitment to the child mediated the relationship between foster parent mind-mindedness and the child's emotional and behavioural problems. Emotional availability could not be included in the analysis due to the small sample $(N=21)$. None of the variables mediated this relationship (all Sobel tests $p$ >.06). Mediators of the relationships between foster parent attachment perceptions and both the quality of the parentchild relationship and the child's emotional and behavioural problems were also investigated. Parent attachment perception was the independent variable and the same hypothesised mediating variables were used, with the addition of mind-mindedness. No significant mediators of these relationships were found (all Sobel tests $p>.06$ ). Some expected mediators (parent depression, anxiety, stress and commitment to the child) were not significant. 
In summary, no variables were found to mediate the relationship between mindmindedness or parent attachment perceptions and the outcome variables

Moderation. To test the hypothesis that foster parent variables may moderate the relationship between mind-mindedness and the child's outcome variables, focused follow-up analyses were also conducted. To examine moderation, the foster parent psychological variables were entered into further regression analyses to investigate the significance of the interaction terms. The hierarchical regression models with significant interaction terms are shown in Table 5. In the first analysis the independent variable was mind-mindedness, the moderating variable was parent attachment perceptions and the dependent varable was the child's emotional and behaviour problems. The direct effect of parent mind-mindedness on the child's emotional and behavioural problems was negative and significant, indicating that parents with higher mind-mindedness had children with fewer emotional and behavioural problems. However, this was qualified by the significant interaction between mindmindedness and parent attachment perceptions. To aid interpretation of these results, the interactions were graphed using ModGraph (Jose, 2013a; see Figure 1).

Table 5 Hierarchical Regression Model Predicting Quality of the Foster Parent-child Relationship and Foster Child Emotional and Behavioural Problems using an Interaction Term

\begin{tabular}{lccc}
\hline Variable & $\boldsymbol{R}^{2}$ change & $\boldsymbol{F}$ for $\boldsymbol{R}^{2}$ change & $\boldsymbol{\beta}$ \\
\hline $\begin{array}{l}\text { Behaviour problems } \\
\text { Step 1 }\end{array}$ & .07 & & \\
$\begin{array}{l}\text { Mind-mindedness } \\
\text { Step 2 }\end{array}$ & & & $-.32^{*}$ \\
$\begin{array}{l}\text { Parent attachment perceptions } \\
\text { Step 3 }\end{array}$ & .05 & 3.82 & .59 \\
$\begin{array}{l}\text { Mind-mindedness x attachment perceptions } \\
\text { Quality of relationship }\end{array}$ & .09 & 4.86 & $-.88^{*}$ \\
$\begin{array}{l}\text { Step 1 } \\
\text { Mind-mindedness }\end{array}$ & & & \\
$\begin{array}{l}\text { Step 2 } \\
\text { Parent attachment perceptions }\end{array}$ & .02 & 1.28 & .21 \\
Step 3 & & & -.55 \\
Mind-mindedness x attachment perceptions & .13 & 5.25 & $.97 * *$ \\
\hline$\quad * p<.05 ; * * p<.01$ & .11 & 6.71 &
\end{tabular}




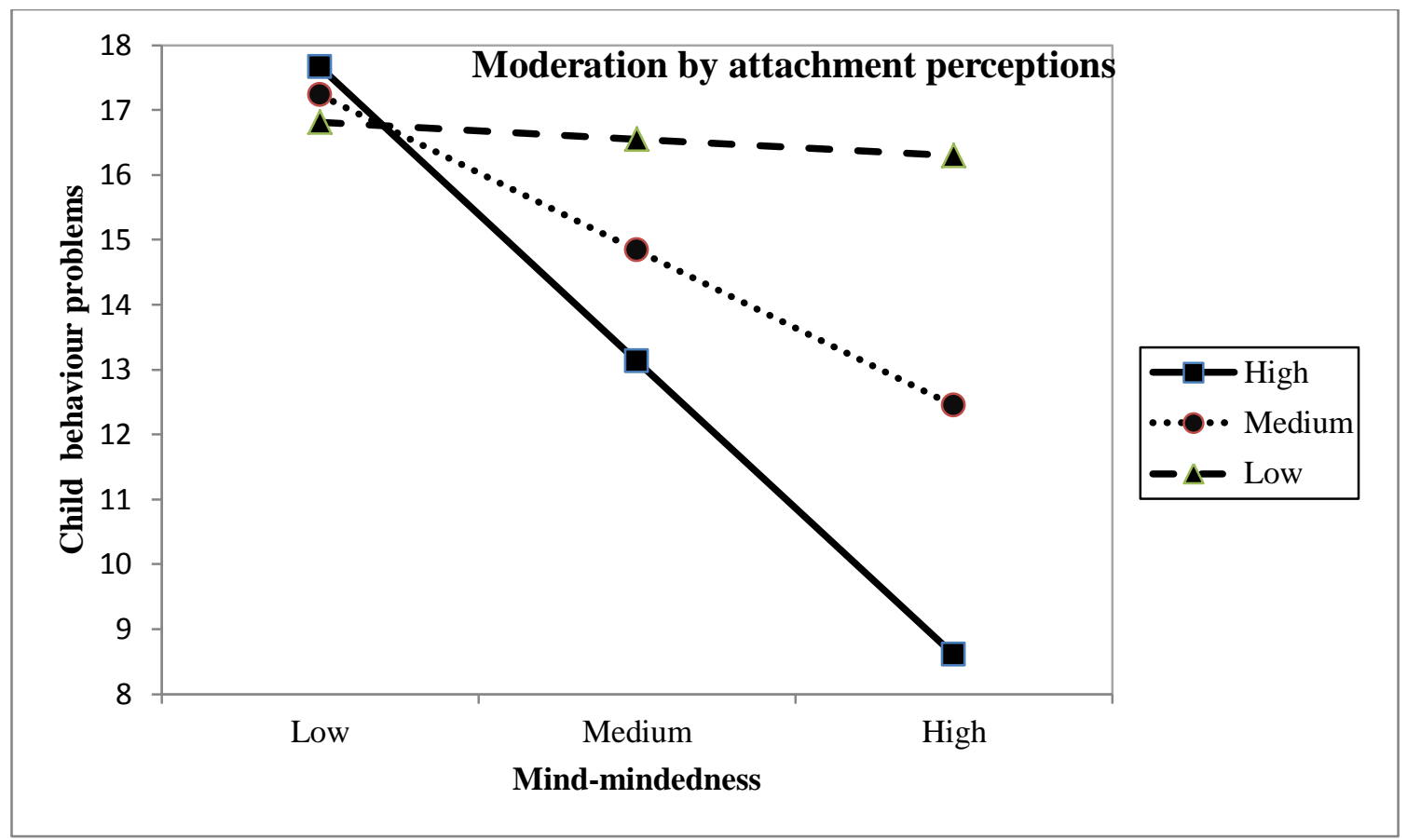

Figure 1. Moderation of relationship between mind-mindedness and the child's behaviour problems, by parent attachment perceptions.

Simple slope analyses indicated that parents with average to highly positive attachment perceptions evidenced a significant negative slope (average PAAQ $t=-2.60, p<.05$; high PAAQ $t=-3.30, p<.01)$ while parents with negative attachment perceptions manifested a non- significant slope $(t=-0.23, p=0.82)$. This suggests that positive parent attachment perceptions amplify the effect on parent mind-mindedness on the emotional and behaviour problems of foster children. That is, whereas a parent with low positive attachment perceptions does not show a relationship between parent mind-mindedness and the child's emotional and behavioural problems, someone with highly positive attachment perceptions shows a strong negative relationship.

Parent attachment perceptions also moderated the relationship between parent mindmindedness and the quality of the parent child relationship (see Table 5 and Figure 2). The direct effect of parent mind-mindedness was positive and non-significant and this was qualified by a significant interaction between parent mind-mindedness and parent attachment perceptions (see Table 5). Using Modgraph (Jose, 2013), simple slope analyses indicated that parents with highly positive attachment perceptions evidenced a significant positive slope $(t=$ $3.01, p<.01$ ) while parents with average to low positive attachment perceptions manifested a non- significant slope (average PAAQ: $t=1.82, p=.07$; low PAAQ: $t=-0.71, p=0.48$ ). 
These results suggest that, as with behavioural problems, positive parent attachment perceptions amplify the effect on parent mind-mindedness on the quality of the foster parentchild relationship.

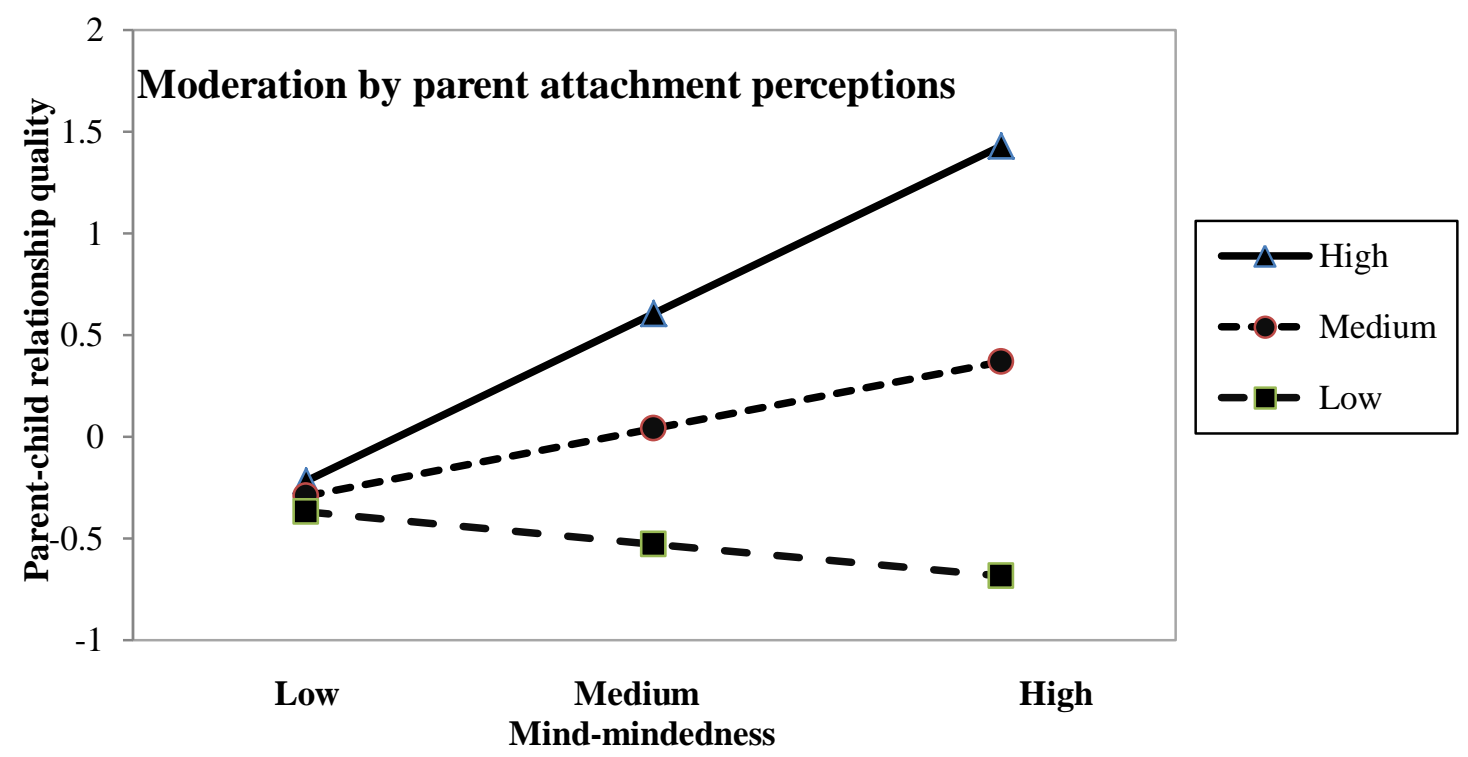

Figure 2. Moderation of relationship between parent mind-mindedness and the foster parent-child relationship, by parent

Note. Figure 2 is virtually a mirror image of Figure 1 due to the valence of the measures, that is, low behaviour problem scores are optimal and high quality of relationship scores are optimal.

To further examine moderation, post hoc analyses were conducted, with the child's gender and age entered into further regression analyses to investigate the significance of the interaction terms. Two significant moderations were found. In the first moderation analysis, the independent variable was parent attachment perceptions, the moderating variable was the child's gender and dependent variable was the quality of the parent-child relationship. The parent attachment perceptions variable was multiplied with the dummy-coded gender variable. The direct effect of parent attachment perceptions was positive and significant and this was qualified by a significant interaction between gender and parent attachment perceptions (see Table 6 and Figure 3). Simple slope analyses indicated that boys evidenced a significant positive slope $(t=4.05, p<.001)$ while girls manifested a non-significant slope $(t=0.85, p=.40)$. This suggests that positive parent attachment perceptions are more strongly related to the quality of the foster parent-child relationship for boys, but not for girls. 
Parents of male foster children show a significant relationship between parent attachment perceptions and the quality of the parent- child relationship but the parents of girls did not.

The same procedure was followed with the childs' emotional and behavioural problems as the dependent variable and the resulting hierarchical regression analysis showed a nonsignificant interaction term $(\beta=.25, \mathrm{p}>.05)$.

Table 6 Hierarchical regression model predicting quality of the foster parent-child relationship and foster child emotional and behavioural problems using an interaction term

\begin{tabular}{lccc}
\hline Variable & $\boldsymbol{R}^{2}$ change & $\boldsymbol{F}$ for $\boldsymbol{R}^{2}$ change & $\boldsymbol{\beta}$ \\
\hline $\begin{array}{l}\text { Behavioural problems } \\
\text { Step 1 }\end{array}$ & .05 & 2.88 & $-.88^{*}$ \\
$\begin{array}{l}\text { Parent attachment perceptions } \\
\text { Step 2 }\end{array}$ & .01 & 0.38 & .02 \\
$\begin{array}{l}\text { Age of child } \\
\text { Step 3 }\end{array}$ & .07 & 4.44 & $.71^{*}$ \\
$\begin{array}{l}\text { Age x attachment perceptions } \\
\text { Quality of the relationship }\end{array}$ & & & \\
$\begin{array}{l}\text { Step 1 } \\
\text { Parent attachment perceptions }\end{array}$ & .13 & 9.04 & $.70^{* * *}$ \\
$\begin{array}{l}\text { Step 2 } \\
\text { Gender }\end{array}$ & .03 & 2.07 & .18 \\
$\begin{array}{l}\text { Step 3 } \\
\text { Gender x attachment perceptions }\end{array}$ & .08 & 5.90 & $-.43^{*}$ \\
\hline
\end{tabular}

Note. ${ }^{*} p<.05 ; * * p<.01$

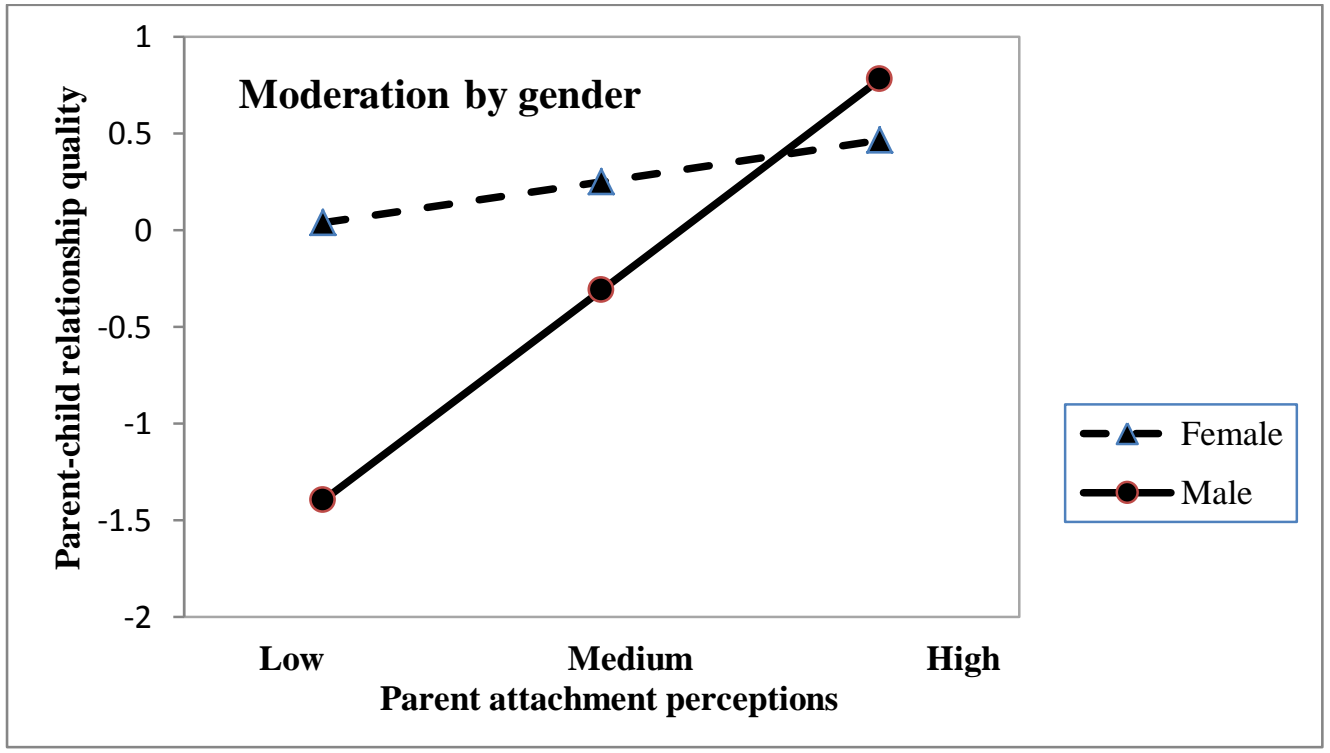

Figure 3. Moderation of relationship between parent mind-mindedness and the foster parent-child relationship, by parent 
In the second post-hoc analysis, the independent variable was parent attachment perceptions, the moderating variable was the child's age and dependent variable was the child's emotional and behaviour problems. The relationship between positive parent attachment perceptions and the child's behavioural problems was negative and significant and the child's age was a significant moderator of this relationship (see Table 6). The direct effect of parent attachment perceptions was negative and significant and this was qualified by a significant interaction between the child's age and parent attachment perceptions (see Table 6 and Figure 4). Simple slope analyses indicated that younger children evidenced a significant negative slope $(t=-2.71, p<.01)$ while average and older children manifested non-significant slopes (average age: $t=-1.93, p=.06$; older age: $t=.05, p=0.96$ ). Parents of younger foster children show a significant, negative relationship between parent attachment perceptions and the child's behaviour problems but the parents of older foster children do not. The parent's positive attachment perceptions are not significantly related to the quality of the foster parent-child relationship with older children.

The same procedure was followed with the childs' emotional and behavioural problems as the dependent variable and the resulting hierarchical regression analysis showed a nonsignificant interaction term $(\beta=-.23, p>.05)$. The sample size was not sufficently large to allow the significant moderators to be entered in a path analysis together.

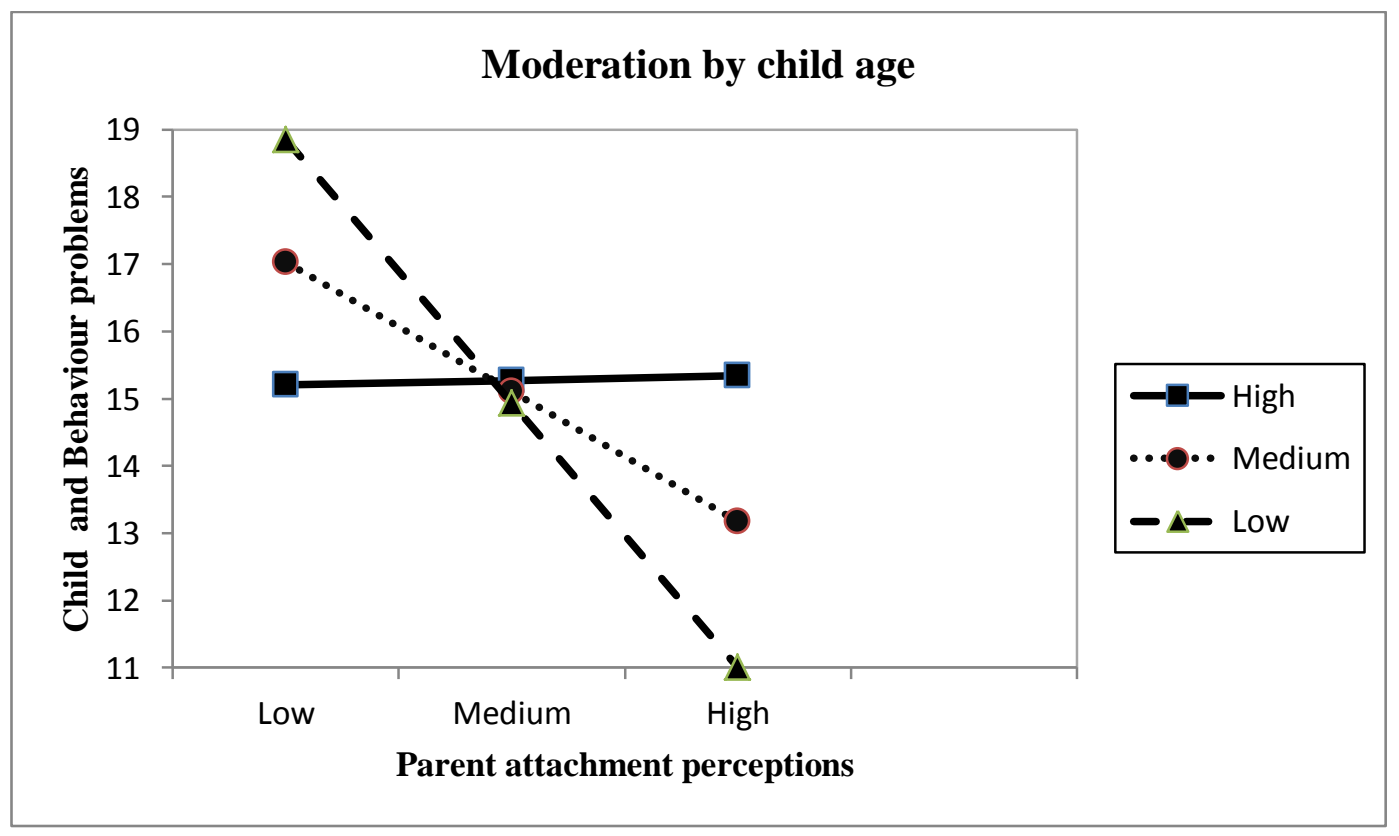

Figure 4. Moderation of relationship between parent attachment perceptions and the quality of the foster parent-child relationship, by child's gender. 
In summary, foster parent attachment perceptions moderated the relationship between mind-mindedness and both child outcome variables. The child's age moderated the relationship between foster parent attachment perceptions and the child's emotional and behaviour problems. The child's gender moderated the relationship between foster parent attachment perceptions and the quality of the parent-child relationship.

Path analysis. In order to consider the regression analyses together in one model path analyses were performed. The path analyses used maximum likelihood estimation and four criteria were used to evaluate the fit of the model. These were a non-significant $\chi^{2}$, a comparative fit index (CFI) of above 0.95 , an incremental fit index of over 0.95 and the root mean error square of approximation (REMSEA) of below .08. Variables were entered into the path analysis on the basis of significant Pearson's correlations and significant beta weights in hierarchical regression. The variables were age placed, number of previous placements, parent mind-mindedness and parent attachment perceptions. Model 1 showed 4 significant paths (see Table 7 and Figure 5) between age placed and the quality of the parentchild relationship, parent attachment perceptions and the quality of the parent-child relationship, parent mind-mindedness and the child's emotional and behavioural problems, and parent attachment perceptions and the child's emotional and behavioural problems. The path between number of previous placements and emotional and behavioural problems was significant only at the .10 level. The amount of the variance of the SDQ accounted for by the model was $14 \%$ and of CPRS was $26 \%$. This model was found to be an acceptable fit to the data $(\mathrm{CMIN} / \mathrm{DF}=1.668, d f=3, p=.172, \mathrm{CFI}=0.97, \mathrm{IFI}=0.98, \mathrm{RMSEA}=.11)$.

Table 7 Regression weights for path analysis model 1

\begin{tabular}{|c|c|c|c|c|c|c|c|}
\hline & & & Estimate & S.E. & $\boldsymbol{\beta}$ & C.R. & $p$ \\
\hline SDQ & $\leftarrow$ & Previous placements & 3.36 & 2.12 & 0.16 & 1.67 & 0.10 \\
\hline SDQ & $\leftarrow$ & Parent mind-mindedness & -11.69 & 5.05 & -0.21 & -2.32 & 0.02 \\
\hline SDQ & $\leftarrow$ & Parent attachment perceptions & -0.79 & 0.35 & -0.27 & -2.26 & 0.02 \\
\hline CPRS & $\leftarrow$ & Age placed & -0.13 & 0.04 & -0.32 & -3.57 & $* * *$ \\
\hline CPRS & $\leftarrow$ & Parent attachment perceptions & 0.23 & 0.07 & 0.39 & 3.46 & $* * *$ \\
\hline
\end{tabular}




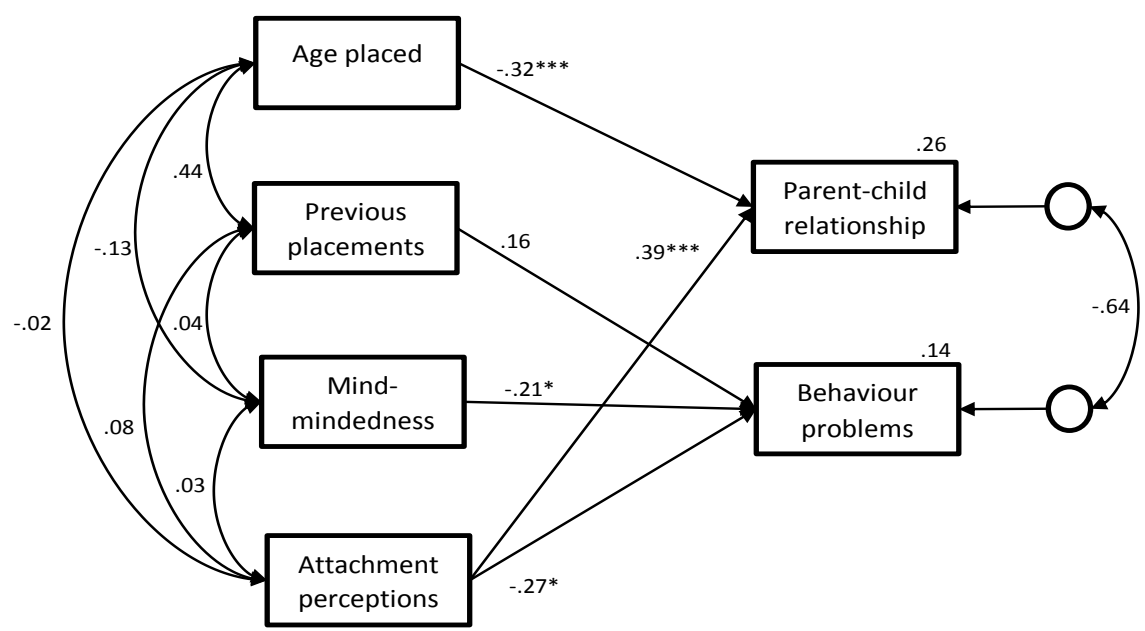

Figure 5. Path analysis model 1.

A further model was tested by removing the non-significant path between number of previous placements and the child's emotional and behavioural problems and adding short term placements. Model 2 showed 5 significant paths (see Figure 6 and Table 8) between age placed and the quality of the parent-child relationship, parent attachment perceptions and both the quality of the parent-child relationship and the child's emotional and behavioural problems and short term placement with both the quality of the parent-child relationship and the child's emotional and behavioural problems. The path between parent mind-mindedness and the child's emotional and behavioural problems was only significant at the .10 level. The model accounted for $21 \%$ of the variance of the SDQ and $31 \%$ of the variance of the CPRS. This model was found to be a good fit to the data $(\mathrm{CMIN} / \mathrm{DF}=.90, d f=2, \mathrm{p}=.41, \mathrm{CFI}=$ $1.00, \mathrm{IFI}=1.00, \mathrm{RMSEA}=.00)$.

Table 8 Regression weights for path analysis model 2

\begin{tabular}{|c|c|c|c|c|c|c|c|}
\hline & & & Estimate & S.E. & $\boldsymbol{\beta}$ & C.R. & $\mathbf{p}$ \\
\hline SDQ & $\leftarrow$ & Short term placement & -5.18 & 1.92 & -.32 & -2.69 & .01 \\
\hline SDQ & $\leftarrow$ & Parent mind-mindedness & -8.79 & 5.15 & -.16 & -1.71 & .08 \\
\hline SDQ & $\leftarrow$ & Parent attachment perceptions & -0.74 & 0.35 & -.26 & -2.15 & .03 \\
\hline CPRS & $\leftarrow$ & Age placed & -0.11 & 0.04 & -.26 & -2.93 & .01 \\
\hline CPRS & $\leftarrow$ & Short term placement & 0.77 & 0.37 & .23 & 2.09 & .04 \\
\hline CPRS & $\leftarrow$ & Parent attachment perceptions & 0.23 & 0.07 & .38 & 3.50 & $* * *$ \\
\hline
\end{tabular}




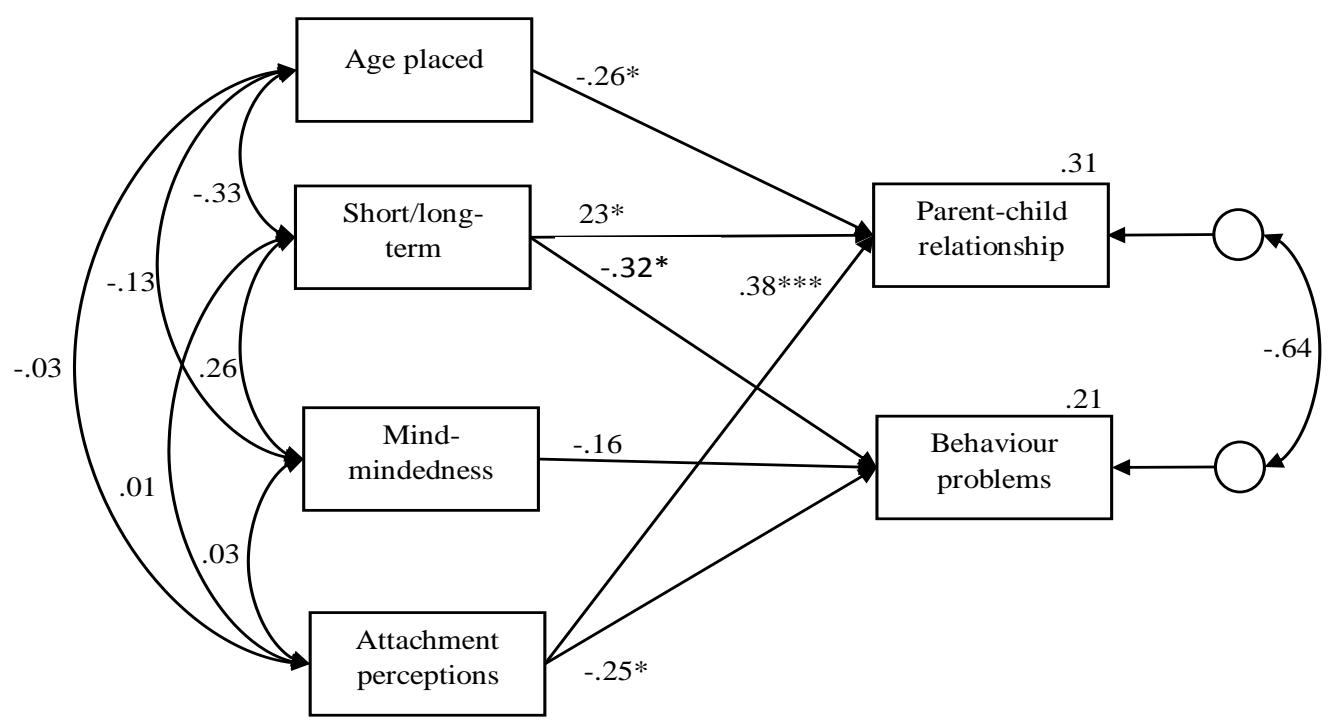

Figure 6. Path analysis for model 2.

In summary, when both child outcome variables were entered together, the best predictors of the child's behaviour problems were short term placements and parent attachment perceptions and the best predictors of the quality of the parent-child relationship were age placed and parent attachment perceptions. Mind-mindedness was only marginally predictive of child behaviour problems in this model.

\section{Discussion}

The aims of the current study were to investigate the inter-relationships between foster child demographic and placement variables, foster parent psychological characteristics and the child's emotional and behaviour problems and the quality of the parent-child relationship. The key variable of interest was foster parents mind-mindedness.

\section{Foster parent mind-mindedness and children's emotional and behaviour problems}

It was predicted that foster parents' mind-mindedness would be inversely associated with the child's emotional and behaviour problems. In line with predictions, foster parents' 
mind-mindedness was negatively associated with the child's emotional and behaviour problems. More mind-minded foster parents had children with fewer emotional and behaviour problems. Although the cross-sectional nature of the study does not allow causal connections to be made, this finding is consistent with mind-mindedness theory, which proposes that parents who comment on their child's thoughts and emotions help the child to understand and predict the behaviour of others, making it easier for the child to regulate their behaviour (Demers et al., 2010b; Meins, 2013). However, it is also possible that more mindminded foster parents perceive the child to have fewer emotional and behaviour problems, as they are more focused on the child's perspective.

A longitudinal study with multiple informants is needed to address this issue. One such study investigated the associations between mothers' mind-mindedness, when the child was 8 months old, and the child's emotional and behavioural problems at 5 years old (Meins, Centifani, Fernyhough, \& Fishburn, 2013). Meins et al. (2013) found a significant relationship for children in a low socio-economic sample, but not a high socio-economic sample. The outcome measure was a combined rating of the child's emotional and behaviour problems from two informants - the child's mother and the child's teacher (Meins, Centifani, Fernyhough, \& Fishburn, 2013). This study provides preliminary data suggesting it is less likely that more mind-minded parents perceive the child to have fewer difficulties.

While Meins et al. (2013) found a relationship between parent mind-mindedness and children's emotional and behaviour problems in a low socio-economic group, but not a high socio-economic group, other research found this association with pre-schoolers in a community, but not clinical, sample (Walker et al., 2012). These two studies suggest that parent mind-mindedness may confer advantages to children's emotional and behaviour problems depending on the level of disadvantage and risk factors for mental health. The current study is the first to show that mind-mindedness is associated with children's emotional and behaviour problems in foster care and in an older age group, up to 13 years of age.

The relationship between foster parent mind-mindedness and the child's emotional and behaviour problems was amplified by foster parent attachment perceptions. There was a strong inverse relationship between parent mind-mindedness and the child's emotional and behavioural problems when parents had highly positive attachment perceptions. When parents had negative attachment perceptions the relationship between mind-mindedness and the child's emotional and behavioural problems was not significant. This finding is discussed below. 
It was predicted that the positive and negative valence of the foster parents' mindrelated comments would be associated with the child's emotional and behaviour problems. Consistent with this hypothesis, parents who made more positive mind-related comments and fewer negative mind-related comments had children with fewer emotional and behaviour problems. It is possible that parents could simply be using more positive descriptors with better behaved children and more negative descriptors with more poorly behaved children. This is unlikely, however, as total positive and negative comments, including behavioural, general, and physical comments, were not related to the child's emotional and behaviour problems and only the valence of the mind-related comments was significant.

Previous research has shown that the positive and negative valence of mind-related comments is associated with parenting behaviour, with differing results. Mothers who made fewer negative mind-related comments, but not more positive mind-related comments, were rated as more sensitive in an observation of mother-child interaction (Demers et al., 2010a). Conversely, mothers who made more positive mind-related comments in the mindmindedness interview, but not fewer negative mind-related comments, were rated as more sensitive to the child's cues (Demers et al, 2010b). Using the interview measure, mothers with more positive mind-mindedness were less hostile toward the child (McMahon \& Meins, 2012). Demers et al. (2010b) have speculated that parents who view their child's mental attributes in positive ways may interpret the child's cues more accurately and respond with more warmth. Other research has shown that parents who are able to discuss both the positive and negative sides of their children are more likely to have securely attached children (Benoit, Parker, \& Zeanah, 1997; Oppenheim, Koren-Karie, \& Sagi, 2001).

In the current study, $22 \%$ of the mind-related comments were negative, compared to only $12 \%$ in a community sample (Demers et al., 2010b). More negative mind-related comments might be expected regarding children in foster care, as negative mind-mindedness is coded for emotions such as sad, anxious, worried and angry, and these emotions are likely to be higher in foster children, due to past experiences of loss (Unrau, Font, \& Murphy, 2011, Unrau, et al., 2008). It is interesting, then, that negative mind-mindedness did predict emotional and behaviour problems and this finding does not support the view that negative comments may be an indicator of the parent having a balanced view of the child.

The interview measure of mind-mindedness is used with children beyond infancy. It differs from the infant observation measure and other measures of parent's mental representations in two important ways. The infant observation measure of mind-mindedness assesses whether the parent's comment is mind-related and also the accuracy of the parent's 
comments to the child, as video-recording allows a comparison of the researcher's interpretation of the infant's non-verbal communication to the parent's verbal response to the infant. A disadvantage of the interview measure is that it has no gauge of the accuracy of the parent's comments. This is also true of other measures of parent mental representations.

These include the Parent Development Interview which assesses representations derived from the parent's description of their current relationship with a specific child (Aber et al., 1985). The Working Model of the Child Interview classifies parent's responses into three categories: balanced, disengaged and distorted (Zeanah, Benoit, \& Barton, 1986). The Insightfulness Interview requires parents to watch a brief video of the child and comment on the child's thoughts and feelings and classifies the comments for openness, complexity etc. (Oppenheim \& Koren-Karie, 2013).

It is likely that these measures are assessing different aspects of parent mental representations compared to mind-mindedness. These measures use qualitative, global judgements of categories such as reflectiveness, the ability to take the child's perspective or how the parent describes the child such as their coherence, in a similar way to the AAI. In contrast, the interview measure of mind-mindedness categorises the type of descriptors parents use in a simple, quantitative way. Meins (1997) argues that mothers' use of mental descriptors are an indicator of their ability to see their child as having an independent mental life but it is also possible they simply reflect a higher focus on the child's mind and emotions. This focus could be helpful in a number of ways, as discussed elsewhere. Some key advantages of the interview measure of mind-mindedness are that it is brief ( 5 - 15 minutes compared to up to 90 minutes), does not require specialised training to score and as the participant is unaware of what the scale is measuring, it is difficult to fake.

\section{Foster parent mind-mindedness and the quality of the parent-child relationship}

Parent mind-mindedness was not associated with the quality of the foster parent-child relationship, although the positive and negative valence of the comments was. Parents made more positive mind-related comments, and fewer negative mind related comments, about children with whom they reported a better relationship. Foster parent attachment perceptions moderated the relationship between mind-mindedness and the quality of the parent-child relationship. There was a strong positive relationship between parent mind-mindedness and the foster parent-child relationship when parents had highly positive attachment perceptions.

The current study found that mind-mindedness and attachment perceptions work in conjunction to predict both children's emotional and behaviour problems and the quality of 
the parent-child relationship. Previous studies have reported a relationship between parent mind-mindedness and state of mind about attachment, as measured by the AAI (Demers et al., 2010b; Arnott \& Meins, 2008). In studies employing the AAI, the parent's state of mind about attachment is derived from a comparison of the parent's experience of their early attachment figures as loving, rejecting and role-reversed with the parent's narrative description of these relationships. The parent's state of mind is measured by their coherence and flexibility in describing these relationships, their inferred truthfulness and their collaboration with the interviewer. In other words, the AAI measures how parents talk about their relationships with their primary caregivers in childhood (Hesse, 2008; Main, Hesse, \& Kaplan, 2005). It is likely that parents' ability to be coherent and flexible in moving their attention between different demands on their attachment thoughts and emotions is related to being able to focus on the mental life and emotions of their child.

On the other hand, a self-report measure such as the PAAQ cannot capture participants' processing of information. The PAAQ focuses on the content of parents' positive and negative emotions and attitudes towards their early relationships. Self-reported attachment perceptions, as measured by the PAAQ, may be related to parent mind-mindedness in a different way compared to the AAI. Firstly, parents who are more negative about their early relationships may also have a propensity to be negative about current relationships. Second, high levels of negative emotions may affect the parent's clarity or willingness to see the child's perspective. It is likely that the PAAQ and the AAI assess different aspects of parents' state of mind about attachment and how it relates to parents' ability to focus on the mental life and emotions of their child. This study is the first to show a relationship between mind-mindedness and a self-report measure of adult attachment.

In summary, foster parent attachment perceptions and mind-mindedness work in conjunction to predict both children's emotional and behaviour problems and the quality of the parent-child relationship. The findings suggest that the parents' processing of their emotions and attitudes toward their own early attachment relationships, together with a focus on the child's mind and emotions, is associated with the child's outcomes.

\section{Foster parent attachment perceptions}

It was predicted that foster parents with more positive attachment perceptions would have children with fewer emotional and behaviour problems and a better foster parent-child relationship. Contrary to this hypothesis, foster parent attachment perceptions did not predict the child's emotional and behavioural problems. However, post-hoc analyses were 
conducted to analyse the potential moderation of the child's age and gender. These analyses showed that the child's age moderated the relationship between foster parent attachment perceptions and the child's emotional and behavioural problems. Foster parents with positive attachment perceptions had children with fewer behaviour problems, only if they were in the youngest third of the sample. The significant interaction effect suggests that parents' current perception of their own attachment relationships in childhood is associated with younger children's behaviour problems.

While the correlational nature of the analyses does not allow conclusions to be drawn about causality, there are several possible explanations for this finding. One possible explanation is that younger children may have less entrenched behaviour problems when they enter the placement, as older children have been found to have higher rates of emotional and behaviour problems (Burns et al, 2004; Steele \& Buchi, 2009). It is also possible that they may have a better response to new caregivers. Although studies have shown that even very young children may have rejecting behaviour towards a new caregiver, they have also shown that younger children are more likely to develop a secure attachment (Bernier et al., 2004; van den Dries et al., 2009) . Older children may also have a number of other influences on their emotional and behaviour problems such as the effects of the child's peer group and more awareness of having different circumstances compared to children in birth families.

It was predicted that foster parent attachment perceptions would be associated with the quality of the parent-child relationship. Consistent with this hypothesis, parents with positive attachment perceptions reported a better relationship with the foster child. Previous studies have shown that mothers' attachment perceptions are related to their mental representations about caregiving and mothers' maltreatment of the child (Cicchetti, et al., 2006; Huth-Bocks et al., 2004).

While foster parent attachment perceptions predicted the quality of the parent-child relationship, moderation analyses showed this relationship was significant for boys but not for girls. This finding is in line with research showing that boys respond differently to caregiving contexts than girls. Attachment disturbances in maltreated infants have been found to be related to the absence of a father in boys but not in girls (Carlson, Cicchetti, Barnett, \& Braunwald, 1989). In addition, fewer boys developed secure attachment with their foster parent when placed in foster care after being in an orphanage, compared to girls (McLaughlin, Zeanah, Fox, \& Nelson, 2012). Secure attachment prevented the onset of internalising behaviour problems for girls in the foster care group but boys in the foster care 
group showed similar proportions of secure attachment and internalising behaviour problems to the group who remained in the insitution.

The relative vulnerability of boys from adverse environments in new caregiving relationships, compared to girls, is an important area of research. Studies using structured diagnostic interviews have consistently found that boys in foster care have higher levels of mental health disorders than girls, particularly conduct problems. Studies using standardised questionnaires do not usually show gender differences, however. Longitudinal research using a baseline measure of the child's emotional and behaviour problems at entry into foster care and relationship formation over the early months of foster care could potentially clarify gender differences. This would require assessing both the foster parent's and foster child's perceptions of the relationship.

\section{Emotional availability}

We hypothesised that parents and children with higher emotional availability would have fewer emotional and behaviour problems and a better quality parent-child relationship. Contrary to this prediction, foster parent emotional availability was not related to either outcome variable and only child involvement with the foster parent was associated with the foster parent child relationship. This finding is contrary to studies showing that parent emotional availability is related to children's agression and behaviour problems (Biringen, Skillern, Mone, \& Pianta, 2005), although it may reflect lack of statistical power. The current study found associations between aspects of foster parents' emotional availability and children's demographic and placement characteristics, such as the age at which the child was placed. While the small sample limits the conclusions that can be made, the finding that parents were more sensitive and less intrusive with children placed at an earlier age is potentially important, as it suggests that foster parents respond to children with different characteristics in different ways and this may be a fruitful area for further research.

\section{Child and Placement Characteristics and the Child's Emotional and Behaviour Problems}

It was predicted that the child's emotional and behaviour problems would be positively related to the child's age, the age at which they were placed, short term placements, the number of previous placements, and types of maltreatment the child had experienced. It should be noted that although specific hypotheses were not made, the relationship between the child's ethnicity and emotional and behaviour problems was also examined. This 
relationship was not significant, indicating that Māori children did not differ from non- Māori children in their emotional and behaviour problems.

Contrary to the first hypothesis, the child's age and the age at which they were placed were not related to their emotional and behaviour problems. This finding is inconsistent with two studies showing a relationship between age placed and children's mental health (Maaskant, Rooij, \& Hermanns, 2014; Tarren-Sweeney, 2008), although the latter used a different variable: the age at which the child first entered care. Although these variables would be expected to correlate, age at first entry to care is likely to be a stronger predictor of children's emotional and behaviour problems than the age the child was placed in the current placement.

Second, in line with expectations, children who were in short term placements had more emotional and behaviour problems. This finding is consistent with research showing that lack of certainty about the future predicts children's mental health (Tarren-Sweeney, 2008a). This study showed that the foster parent's expectation that the child would be reunited with his or her birth parents and lack of placement permanency predicted the child's mental health. Indirect support is also provided by studies showing that children who are adopted, and thus have legalised permanency, have fewer mental health problems than those who remain in foster care (Proctor et al., 2011; Rushton, 2004). Tarren-Sweeney (2008a) proposes an attachment hypothesis to explain this finding, "namely that, placement security and permanency directly affect the attachment security of children in long term care, via their perceptions of permanence, as well as indirectly, via the influence of placement security on their carer's attachment systems" (p. 20).

Third, the number of previous placements, as reported by foster parents, was also associated with the child's emotional and behaviour problems. Children who had had more placement changes had more emotional and behavioural problems. This finding is consistent with studies which have shown a bi-directional relationship between placement disruption and children's emotional and behavioural problems. These studies have shown that placement instability is associated with children's behaviour problems, independent of the child's behaviour problems at entry into foster care (Newton et al., 2000; Osborn, et al., 2008; Rubin, O'Reilly, Luan, \& Localio, 2007). Placement instability has also been found to be related to children's difficulties with impulse control and social withdrawal (Lewis, Dozier, Ackerman, \& Sepulveda-Kozakowski, 2007; Unrau et al., 2008).

Fourth, children who experienced more types of maltreatment, as reported by foster parents, had more emotional and behaviour problems. This finding is consistent with 
research showing that specific types of maltreatment predict emotional and behavioural problems in foster children (Raviv et al., 2010: Tarren-Sweeney, 2008). Research has also shown that children's emotional and behaviour problems are strongly related to their length of time in an adverse environment prior to entering care (Berzin, 2008; Ford et al., 2007). Maltreatment is a strong predictor of emotional and behaviour problems in children through a number of mechanisms including increases in cortisol levels, decreases in emotion regulation and disruptions of attachment relationships (Cicchetti \& Valentino, 2006, Fearon et al., 2010; Fisher et al., 2006).

Fifth, the expectation that the child's emotional and behaviour problems would not be related to the duration of placement was supported. This supports previous research showing that foster children's behaviour problems do not tend to improve over their time in foster care (Biehal, Ellison, Baker et al., 2011; Wilson, 2006).

In conclusion, there were a variety of associations between children's demographic and placement variables and the child's emotional and behaviour problems. It is unclear whether these associations can be explained by the effect of the child's demographic and placement characteristics on their emotional and behaviour problems and the foster parent-child relationship, or whether foster parents respond to children with different characteristics in varying ways, which in turn affects the child's outcome. Again, a longitudinal and multiinformant research is needed to illuminate this question.

\section{Child and Placement Characteristics and the Quality of the Foster Parent Child Relationship}

It was hypothesised that parents would report a positive relationship with younger children, those placed at a younger age, those who had experienced fewer placements and those who were in a long-term placement and had been in their placement for a longer time. It should be noted that the relationship between the child's ethnicity and the quality of the foster parent-child relationship was also examined and was found to be non-significant. This indicates that foster parents did not report a significantly different relationship with Māori children than non-Māori children.

Consistent with the first hypothesis, parents reported a better parent-child relationship with children who were younger and had been placed at a younger age. Relationship formation in foster care has been investigated with very young children's approach and avoidance of the foster parent and the child's attachment security. These studies have shown that children placed under a year old are more likely to approach the foster parent when 
distressed and show more secure behaviour than children placed at over a year of age (Stovall \& Dozier, 2000; Stovall-McClough \& Dozier, 2004). This finding was not accounted for by the number of previous placements the child had experienced or the child's previous exposure to risk.

Second, as predicted, foster parents reported a better parent-child relationship with children who had fewer previous placements and were in a long term placement. This finding is contrary to two studies which have investigated this link found no significant relationship between attachment security and the number of previous placements (Altenhofen et al., 2013; Joseph et al., 2013), although this may be due to low numbers of placement changes in both studies. It is possible that the relationship between the child's previous placements and the foster parent-child relationship might be affected by foster children's feelings of insecurity, fearfulness and anger when moving placements and social withdrawal as a consequence of experiencing many losses (Unrau, et al., 2011, Unrau et al., 2008).

Third, foster parents also reported a better parent-child relationship with children who were in a long term placement. This facet of the child's placement has not been examined in relation to the foster-parent child relationship or attachment security and possible links to these outcomes are discussed above.

Fourth, contrary to expectations, placement duration did not predict the quality of the parent-child relationship. This finding is suprising as children require a continuous relationship to build trust in new caregivers (Golding, 2011; Schofield et al., 2012). Foster parents can also provide a secure base for the child to explore and learn in the context of reliable close relationships (Schofield \& Beek, 2005a, 2005b). It would therefore be expected that the parent-child relationship would improve over time. A series of studies by Mary Dozier and her colleagues have shown that young children in foster care often display rejecting behaviours at the beginning of placement and that these become more organised and secure over time, espectially if the foster parent has a secure state of mind about attachment (Dozier et al., 2001; Stovall \& Dozier, 2000; Stovall-McClough and Dozier, 2004). However, in this study, we measured emotional closeness and conflict in the parent-child relationship, rather than attachment security.

In summary, a variety of associations were found between children's demographic and placement variables and the quality of the parent-child relationship, although the predicted association between the duration of the child's placement and the quality of the parent-child relationship was not found. 


\section{Other foster parent characteristics}

Foster parents' commitment to the child was correlated as predicted with both outcome variables. The more committed the foster parent was to the child, the fewer emotional and behaviour problems the child had and the better the quality of the parent-child relationship. Previous studies have shown that foster parent commitment is associated with children's emotional and behaviour problems and the foster parents" "delight" in interaction with their foster infants (Bernard \& Dozier, 2011; Lindhiem \& Dozier, 2007). In the current study, foster parent commitment was associated with long term placements. Somewhat surprisingly, neither expected length of placement or parent commitment mediated or moderated relationships between placement characteristics, foster parent psychological functioning and the child's outcome variables.

\section{Strengths and limitations}

The main strength of the study was the use of in-depth phone interviews with foster parents and a comprehensive battery of questionnaires which allowed an examination of variables which have not been previously studied with this population of children. These variables included mind-mindedness, a self-report measure of adult attachment and the quality of the parent-child relationship. The interviews and questionnaires are a valuable way to place less reliance on administrative data, which many studies have relied on (Pritchett et al., 2013).

The main limitation of the study was the use of a small, non-random sample due to sampling difficulties, including the complex procedures for obtaining consent, the low response to general invitations by letter or email to participate, and data collection being disrupted by a major earthquake in Christchurch, New Zealand. Participation rates increased when the researcher made a presentation of the study at foster parent gatherings and discussed participation with potential participants. These factors suggest that the sampling procedure may be biased towards foster parents who are more involved in foster parent education, such as regional gatherings and conferences.

However, the sample included rural and urban foster parents from all parts of New Zealand and they had had a wide range of education and experience in foster care. The rate of child behaviour problems is also comparable with other studies and negative indices such as negative mind-mindedness were higher than in studies of birth parents. There was a low rate of Māori foster parent participation but an over representation of Māori foster children, 
which is comparable to the foster care population in New Zealand. There was also an overrepresentation of non-kin foster care compared to family/whānau foster care. A further limitation was the wide age range used, partly in response to the sampling difficulties. The children's ages ranged from 2 - 13 years, encompassing a wide range of developmental changes. However, age was associated with very few variables. Another limitation was the use of one informant, namely the foster parent who spent the most time with the child. Ideally the child's teacher and social worker would have provided information. Where birth parents provided consent for their child to participate in the EAS videotaping, they also provided consent for the childs' teacher to complete the SDQ. However, numbers were insufficent and this questionnaire was dropped.

A strength of the study was using a standardised observational measure of parent-child emotional availability but this strength became a limitation due to the small number of birth parents who could be contacted to give consent for the child to participate in the research. Had this not been a problem, the relationship between parent sensitivity and children's emotional and behaviour problems in foster care could have been clarified for the first time. Lastly, several variables ( the number of types of maltreatment, short term placements and number of previous placements) were foster-parent-reported, rather than a standardised measure of information contained in the child's file, or social-worker-reported.

\section{Conclusions}

The most important finding of this study is that foster parents' mind-mindedness and self-reported attachment perceptions work in conjunction to predict foster children's behaviour problems and the quality of the foster parent-child relationship. This is a key finding in several ways. First, it shows the complexity of the associations between foster parent and child variables in foster care. Second, it extends previous findings to include foster parents' mental representations and a wider child age range. Third, it shows that foster children's outcomes are not pre-determined by their previous adverse experiences. The findings suggest that aspects of foster parent psychological functioning work together in complex ways which, if better understood, have the potential to be harnessed for the benefit of foster children. The findings suggest it would be fruitful to shift focus from foster parents being seen as providers of safe and long term care, to them also being a resource for the amelioration of foster childrens' problems.

Another important finding is that foster parent psychological functioning may have different associations for boys and girls, and younger and older children, and that foster 
parent mind-mindedness and, tentatively, sensitivity may be associated with demographic or placement characteristics such as the child's age and the age at which they were placed. This finding has implications for foster care practice as it suggests that the screening and matching of aspects of foster parent psychological functioning and the type of child they provide care for may be of critical importance.

While firm conclusions are limited by a cross-sectional design, a small sample and data from a sole informant, these findings are consistent with other studies examining mindmindedness in other populations and parent attachment perceptions using different measures. These findings support an ecological transactional view in that foster parent and child characteristics interact in complex ways and mutually influence each other. These findings have implications for both foster parent selection and training. 


\section{Chapter Five-Training programmes and therapeutic intervention in foster care}

A number of diverse training programmes and therapeutic interventions have been developed or adapted for use in foster care. Foster care itself is the most common intervention for maltreatment in Western countries. It has been suggested that child welfare has focused on safety and permanency and that the therapeutic aspect of foster care has not been fully explored (Horwitz et al., 2010; Leve et al., 2012). Leve et al. (2012) argue that:

Historically, child welfare systems have aimed to address child safety, permanence/stability, and well-being, with a primary focus on the first two, and much less on the third; a child's well-being is often assumed to be addressed by mental health, developmental and education services (p. 12).

Given that many children in care will not receive mental health services, foster care in and of itself can be regarded as an intervention with the potential to assist children in care to overcome their difficulties (Burns et al., 2004; Nilsen, 2007).

Interventions for foster families differ in offering a) an intervention for the foster parent, foster child, or both, b) an introductory programme focusing on child welfare policies and safe care, a psycho-educational or parent training focus, and c) whether the programme has a group focus or is individually tailored. There has been little recent focus on individual therapy for foster children who have experienced maltreatment and trauma. Recent reviews focus on interventions for foster parents with little mention of individual therapy for children in foster care (e.g., Craven \& Lee, 2008; Leve et al., 2012; Rork \& McNeil, 2011), even though interventions have been developed for maltreated children (Cohen, Mannarino, \& Deblinger, 2006). One possible reason is that the effectiveness of interventions for maltreated children has been evaluated with respect to foster children in only two studies. A more likely reason, however, is that insufficient attention has been paid to trauma in foster care research and the mental health assessments of foster children (Ai et al., 2013).

Four main types of interventions have been researched in foster care: introductory training programmes, parent training programmes based on social learning theory, wrap around programmes including Multi-dimensional Treatment Foster Care, and programmes based on attachment theory. In this chapter, I will discuss the research limitations in this area and the evidence for the effectiveness of the four types of intervention in foster care. 


\section{Limitations and Issues in Foster Care Research}

There are a number of barriers or complications in evaluating interventions in the foster care population. The mobility of foster children makes treatment research difficult as many children may leave the care of the foster parent during the intervention or follow-up period. This change of caregiver may mean the child's data are discarded or a second person completes the post-test and follow-up questionnaires (Leve et al., 2012). Brief mental health screens, such as the Child Behaviour Checklist and the Strengths and Difficulties Questionnaire, may not capture the complexity of foster children's problems (TarrenSweeney, 2008b). Changes in more severe behaviours, such as sexualised and suicidal behaviours, may be missed by these measures. Reviews have criticised the reliance on foster parent report and recommend that data is collected from the child, foster parent, case worker and teacher (e.g., Rork \& McNeil, 2011).

In the main, foster care research makes the assumption that foster children will respond to their parents completing a parent training, in the same way that biological children do. The results of foster care interventions are compared to those in the general population (e.g., Turner, Macdonald, \& Dennis, 2007). It is likely, however, that the complex problems foster children have may require longer, more intensive programmes to effect change (Feather \& Ronan, 2009). Foster children often have emotional, neurobiological and traumatic effects of prior maltreatment, multiple placement moves and loss of their biological family. These difficulties require addressing. An introductory or parent training programme, especially if not developed or adapted for children in care, seems unlikely to result in a great deal of behaviour change for the child. A wide range of interventions have been developed to address the needs of maltreated children (Horwitz et al., 2010; Toth et al., 2013). Ai et al. (2013) have argued that it is crucial these evidence-based programmes become routinely available for foster children.

Foster care intervention research aims to decrease children's emotional and behaviour problems, especially externalising behaviour problems, but there is little theoretical explanation for the prevalence and persistence of externalising behaviours in foster children (Biehal, Ellison, Baker, et al., 2011; Lawrence et al., 2006; Wilson, 2006). Targets of intervention require clear explanations of the likely genesis of the problem. Associations amongst externalising behaviour problems, disorganised attachment and maltreatment may be pertinent (see Chapter Three; Fearon, et al., 2010). In other words, children's externalising behaviour problems may be linked to their inability to approach a new caregiver to meet their 
emotional needs, due to their experiences with a maltreating caregiver, and to the effects of maltreatment such as role modeling and emotion regulation (Cicchetti \& Valentino, 2006). These associations may provide possible targets for the development of further foster care interventions, for example, focusing on the parent-child attachment relationship and trauma in older children.

For many of the reasons outlined above, behaviour problems may be more intractable in foster children than those in the general population. This is a possible explanation for poor results, rather than the ineffectiveness of the programmes. Some studies of interventions in foster care have shown almost no change on any measure, no significant differences on behavioural outcomes or no significant differences on any measure outside of knowledge of the programme content (Minnis et al., 2001; Pithouse, Hill-Tout, \& Lowe, 2002; Turner et al., 2007).

Little attention has been paid to targeting the skills and knowledge needed by different foster parents. The same intervention is often delivered whether foster parents have years of experience or are new to fostering, whether the child is having behavioural and emotional difficulties or not, and regardless of the age of the child or young person. Little attention is paid to the fact that many children in care have developmental conditions or psychopathology, which are likely to interfere with the efficacy of any one intervention. Some of these conditions cannot be cured and need to be managed, including low intellectual functioning, foetal alcohol syndrome and brain damage from physical abuse. The question of when to intervene has also been little considered. Most interventions occur close to the beginning of the placement with the advantage of maximising possible benefit but with the disdvantage that results may be confounded by the expected disruptive behaviour of the settling in period.

In conclusion, interventions targeting children in care need to address a wide array of significant problems in disabilities, educational achievement; internalising and externalising behavior problems, post-traumatic stress disorder and complex trauma and attachment relationships.

\section{Introductory programmes for new foster parents}

The most widely implemented training programmes for foster parents are introductory programmes to foster parenting and often include information on being a foster parent, the decision to provide foster care, policies and procedures of the fostering agency, and safe care. 
These programmes can be completed by foster parents before they have a child placed with them or are conducted within the first few months of providing foster care. This creates a problem for researchers about which outcomes to measure, as the child is either not yet in the placement or at the beginning of the placement.

Studies have examined the effectiveness of foster care preparation programmes, such as Model Approach to Partnerships in Parenting (MAPP) and Foster Parent Resources for Information, Development and Education (PRIDE), which are required of foster parents in large parts of the US. There is little empirical support for these progammes (Dorsey et al., 2008). For example, Puddy and Jackson (2003) compared 62 foster parents who completed the MAPS programme, with a control group of 20 foster parents, and found significant differences in only four out of a total of 12 goals and in three out of 22 basic parenting skills. Puddy and Jackson concluded that the MAPS programme is inadequate for the range of skills foster parents need. Where programmes have shown positive results, the questionnaires used have been developed for the study, are limited to topics taught on the programme and have little evidence of validity (e.g., Christenson \& McMurtry, 2009).

In summary, introductory programmes focusing on policies and procedures are designed to keep children physically safe, with little content on the emotional and psychological needs of children in foster care. These programmes have been rolled out with little empirical research and have been strongly criticised (Dorsey et al., 2008).

\section{Individual therapy for trauma}

Trauma Focused Cognitive Behaviour Therapy (TF-CBT) emphasises a set of skills based on cognitive-behavioural principles, including psycho-education, affect modulation, cognitive coping, trauma narrative, and parenting skills (Cohen et al., 2006). Of the individual therapies available for children who have been traumatised, TF-CBT has been shown to be the most effective (Leenarts, Diehle, Doreleijers, Jansma, \& Lindauer, 2013). Little research has included foster children and children who have complex trauma and multiple abuse, however (Cohen et al., 2006). A series of small pilot studies has shown that TF-CBT can be effective with maltreated children with a child welfare file, but in order to show treatment effects, this intervention may take up to 45 face to face and booster sessions with the child, parent and/or caregiver and case worker (Deblinger \& Heflin, 1996; Feather \& Ronan, 2009). 
Using cognitive behavioural methods to address trauma in foster chldren, Taussig and Culhane (2010) conducted a randomised controlled trial with 156 maltreated children aged 911 years old in foster care, and found a small effect size for the reduction in traumatic stress symptoms. Weiner, Schneider, and Lyons (2009) evaluated three types of treatment for different ethnic groups and age groups: Child-Parent Psychotherapy for children up to 6 years, TF-CBT for school aged children and Structured Psychotherapy for Adolescents Responding to Chronic Stress with 133 maltreated children in foster care aged between 3 and 18 years. They found all three treatments to be equally effective with African American, Hispanic and White children. A limitation, however, was that the study lacked a detailed analysis of outcomes, a control group and a follow-up.

Two studies have shown that TF-CBT can enhance other interventions, and itself be enhanced by an engagement component. Individual TF-CBT and elements of Multidimensional Treatment Foster Care (MTFC) were used to enhance a programme for children and youth aged between 2 and 21 years (Farmer et al., 2010). The study found small-tomoderate improvements in the children's emotional and behavioural problems, personal strengths and the child's daily problem behaviours. Dorsey et al. (2014) compared 22 foster children who received TF-CBT as usual to 25 foster children who received the same TF-CBT intervention, augmented with an engagement intervention. This brief engagement intervention focussed on barriers to attending and the participants' goals. The study showed that an engagement component did enhance treatment attendance and completion, and the participants also had reduced post-traumatic symptoms, depression and behaviour problems. However, conclusions are limited by the absence of a control group. In summary, TF-CBT is one of the only interventions, utilising individual therapy with the child, to have been researched with foster children but more randomised control trials are needed. Issues of appropriate dosage also require investigation, as empirically supported programmes may take longer and require adaptation for foster children.

\section{Programmes based on social learning theory}

Researchers have evaluated cognitive behaviour therapy programmes for foster parents and manualised parent training programmes such as the Incredible Years Programme (Linares, Montalto, Li, \& Oza, 2006) or Parent-Child Interaction Training (Timmer, Urquizer, \& Zebell, 2006). These programmes have either been devised for foster parents or for the general population, and are utilised either in the standard form or modifed for the 
foster parent population. These parent trainning programmes "all share a common theoretical basis (social learning theory) and incorporate behavioral, cognitive and developmental principles and concepts" (Sanders, Kirby, Tellegen, \& Day, 2014, p. 338). There have been mixed results using these programmes in foster care. These have included studies which have not found effects on the intervention target such as externalising behaviour, but have found effects on other outcomes (e.g., Linares et al., 2006).

A Cochrane review found six randomised controlled trials of behavioural and cognitive behavioural interventions, involving 463 foster carers (Turner et al., 2007). The review concluded that these interventions had little effect on the foster children's psychological functioning, extent of behaviour problems, and interpersonal functioning. There was also little effect on carers' behaviour management skills, attitudes, and psychological functioning. These findings are surprising given the robust findings of the effectiveness of these programmes with birth parents (Horwitz et al., 2010; Toth et al., 2013). Turner et al. concluded that programmes should directly address a wider range of concerns, such as children's development, trauma, and attachment relationships; that research should include more sensitive outcome measures over a longer time period; and that programmes may need to be more intensive or specialised.

The Incredible Years Program (Webster-Stratton \& Reid, 2010) and Parent-child Interaction Therapy (Eyberg \& Funderbunk, 2011) have both been utilised in foster care. Unexpectedly, results for the Incredible Years Program in foster care have been contradictory. Firstly, a randomised control trial with 29 foster parents found significant decreases in children's problem behaviour, compared to a waitlist control group of 17 foster parents. Only parents in the control group showed significant improvement in self reported parenting strategies, without an accompanying decrease in problem behaviour in the children (Bywater et al., 2011). Secondly, a study comparing intervention effects on both the birth parents and foster parents of 128 neglected children placed in foster homes found only a marginally significant effect on children's externalising behaviours at follow-up, but did find significant differences in two of the five parenting components at follow-up (Linares et al., 2006). Thirdly, children who completed the Child Training component of the Incredible Years Program, as well as the control group, were reported by foster parents to show decreases in physical aggression over time but teachers reported little change in either group. Unexpectedly, the control group, but not the treatment group, had a significant increase in self control. The authors noted that there were baseline differences in ADHD between the 
groups, fewer sessions than usual, and they note that parents may need to be involved to facilitate change.

Parent-child Interaction Therapy (PCIT) is similar to group parent training programmes in that it is based on social learning theory but it is delivered in a different way in that "... it incorporates both parent and child within the treatment session and uses live and individualized therapist coaching..." (Timmer et al., 2006, p. 3). Both a group of 75 foster parents and children, and a group of 98 non-abusive biological parents and children, reported a significant decrease in the children's behaviour problems and in parenting stress after completing individual PCIT for their children's clinically significant behaviour problems (Timmer et al., 2006). Participants were not randomised and there was no control group, however. Parent training programmes have been found to be effective in preventing and treating child maltreatment, especially Parent-Child Interaction Therapy, the Incredible Years Programme and Triple $\mathrm{P}$, the last of which has now been tested in over 40 randomised control trials (Horwitz et al., 2010). These programmes have potential for helping foster parents but have only begun to be evaluated with this population.

Summary. Cognitive behavioural and parent training programmes have been shown to be highly effective with biological parents but have shown mixed results in foster care. When studies do not include broadband, standardised measures such as the SDQ and the CBCL, treatment effects may be missed. Unexpectedly poor results with the Incredible Years Program, which is a well-established evidence-based programme, raises the issue that programmes designed for foster care may need to be qualitatively and quantitatively different from those in the general population. Research is needed on the level, intensity and focus of effective parent training programmes needed in foster care. The individualised PCIT programme has shown preliminary promising results, which require further replication with a randomised controlled trial.

\section{Programmes developed for foster care}

KEEP Intervention. Programmes specifically developed for foster care have been more successful. Chamberlain and her colleagues developed a pre-school adaptation of their successful Multi-dimensional Treatment Foster Care programme for adolescents, described below, to create a programme for all children entering a new foster placement with nonrelated foster parents or kinship carers. This intervention is called KEEP (Keeping Foster Parents Trained and Supported). Participants receive "16 weeks of training, supervision and 
support in behavior management methods" (Price et al., 2008, p.68). There have been three papers on the same randomised control trial of the KEEP programme with a large sample of 359 families in the intervention group and 341 in the control group. Two different outcome measures have been examined (Chamberlain, Price, Leve et al., 2008; Chamberlain, Price, Reid, \& Landsverk, 2008; Price et al., 2008).

Price et al. (2008) found that children in the intervention group had almost double the chance of having a positive exit from the foster placement (17 versus 9\%) through increased rates of re-unification with the child's biological parents or relatives and adoption. The intervention also mitigated the effects of multiple placements, in that the risk of placement disruption for children with multiple placements decreased. Chamberlain, Price, Leve, et al. (2008) found that the intervention decreased the child's daily problem behaviours to a greater extent than occurred for children in the control condition. However, the effect size was small, with a decrease from a mean of almost 6 daily problem behaviours to almost 4.5. Mediation analyses showed that the improvement in children's behaviour problems was partly due to an increase in parent positive reinforcement, especially for children with high pre-intervention behaviour problems. Price, Roesch and Walsh (2012) showed that KEEP reduced children's daily problem behaviours at all levels of baseline problem behaviours and could be effectively implemented by an independent community agency. A small study using an adaptation of KEEP with 18 foster parents in the intervention group and 13 in the usual care group found a significant decrease in parent reported externalising problems relative to the control group, with a medium effect size, but little effect in expected parenting behaviours such as praise (Leather, Spielfogel, McMell, \& Attkins, 2011).

It is noteworthy that most studies of KEEP have used the Parent Daily Report Checklist (PDR) developed by Chamberlain and Reid (1987) for use in an evaluation study of Multidimensional Treatment Foster Care. Apart from Leathers et al. (2011), studies of KEEP have not used standardised measures of child behaviour problems and mental health, such as the Child Behaviour Checklist (Achenbach \& Rescorla, 2000) or the Strengths and Difficulties Questionnaire (Goodman \& Scott, 1999). This limits conclusions which can be drawn about the impact of KEEP on children's behaviour problems, and no studies have compared the PDR, which is a count of daily problem behaviours, and the standardised measures.

In a novel application incorporating KEEP, DeGarmo, Reid, Fetrow, Fisher and Antoine (2014) developed the Pathways Home Foster Care Reunification Intervention with the biological mothers of children in care, most of whom had substance abuse problems. The aim of the intervention was to improve children's behaviour when they returned home to their 
biological family after their first placement in foster care. The study showed that parenting strategies and problem behaviour improved over time and the intervention was especially beneficial for mothers who had drug and alcohol cravings.

Fostering Changes. This group programme was developed in the UK and is based on social learning theory and attachment theory. In a small, uncontrolled pilot study, Warman, Pallett and Scott (2006) found significant differences in carer-reported problems, the quality of the attachment relationship, carer efficacy and the emotional problems sub-scale of the SDQ. There was no significant difference in the children's overall emotional and behavioural problems, however. Briskman et al. (2012) conducted an randomised controlled trial with 34 foster parents in the treatment group and 29 in the control group. Again, significant improvements were found for for carer-reported problems, the quality of the attachment relationship and carer efficacy. There was no significant decrease in the children's emotional and behavioural problems, but as the control group increased on this measure, there was a difference between the treatment and control group.

In summary, programmes based on social learning theory, which are specifically developed for foster parents, appear to be more effective than programmes developed for the general population. Effect sizes tend to be small, some studies have not used standardised mental health questionnaires and several papers may be published from the one study, making comparison difficult. The results suggest that parent training may be less effective in foster care than in the community.

\section{Treatment foster care}

Reviews of treatment foster care found only five randomised control studies meeting their criteria, four of which evaluated the effectiveness of MTFC (Macdonald \& Turner, 2008, 2011). One of these reviews concluded that although the studies were limited in number and scope that treatment foster care may be a "...useful intervention for children and young people with complex emotional, psychological, and behavioural need, who are at risk of placements in non-family settings that restrict their liberty and opportunities for social inclusion" (MacDonald \& Turner, 2008, p.1). The authors note the importance of addressing emotional and psychiatric problems alongside delinquency.

Multi-dimensional treatment Foster Care. The Oregon Social Learning Centre developed a multi-faceted programme, known as Multi-dimensional Treatment Foster Care (MTFC), to decrease the chances of at risk youth entering residential care or prison. The 
programme was first developed for youth leaving a psychiatric unit, youth referred by the courts and children and youth in foster care. The six to nine month comprehensive programme uses an array of interventions, including parent training, weekly supervision and support for the foster parents, weekly individual therapy for the youth, and mental health consultations where necessary. According to Chamberlain (2003) the key components include consistent and predictable forms of discipline, close supervision and separating the young person from similarly acting out peers.

Two more recent randomised control trials have shown that MTFC is effective with both female and male adolescents in the juvenile justice system. These studies showed a significant reduction in the number of days girls spent in detention centres and higher attendance at school. The pregnancy rate was halved at 24 month follow-up: $27 \%$ of the girls in the MTFC group reported a new pregnancy, compared to $47 \%$ of girls in the control group (Chamberlain, Leve, \& DeGarmo, 2007; Kerr, Leve, \& Chamberlain, 2009; Leve \& Chamberlain, 2007). Significant decreases in arrest rates, self-reported violence and selfreported substance abuse have also been found for male adolescents, up to 18 months followup (Eddy, Bridges, Whaley, \& Chamberlain, 2004; Smith, Chamberlain, \& Eddy, 2010).

Recent studies by independent researchers have examined the portability of MTFC in countries other than the US. Replication studies have been carried out in Sweden and the UK, with mixed results. Girls with chronic delinquency were found to have decreased offending, violence and self harm, after taking part in MTFC, with similar effect sizes for a US programme and a UK programme (Rhoades, Chamberlain, Roberts, \& Leve, 2014). In Sweden, initial significant decreases in externalising behaviour problems and depression after MTFC, in at risk young people with conduct disorder, were not maintained by the end of the second year of follow-up (Hansson \& Olsson, 2012; Westermark, Hansson, \& Olsson, 2011). Similarly, a UK study with youth offenders found positive differences in offences and living with family at one year after entry into MTFC, but not at one year after exit from the programme (Biehal, Ellison, \& Sinclair, 2011). Another UK study found no benefit of MTFC over usual care with youth in unstable placements and who had complex or severe emotional difficulties. The authors suggest that “...this style of intervention may be less appropriate for the group of young people in care with primarily attachment, interpersonal or emotional needs" (Green et al., 2014, p. 219).

Despite these findings in countries outside the US, MTFC has been found to be a costeffective alternative to residential care and it has been accepted as meeting "top tier" evidence for effectiveness by the Coalition for Evidence-based Policy (Rhoades, Leve, 
Harold, Kim, \& Chamberlain., 2014). In addition, two long term follow-ups into early adulthood, the second up to 9 years later, showed significantly greater decreases in drug use, depressive symptoms, suicidal ideation and also greater resilience to partner drug use in the MTFC group than in the usual care group (Rhoades, Leve et al., 2014; Kerr, DeGarmo, Leve, \& Chamberlain, 2014).

In summary, MTFC has been found to be a highly effective progamme for youths who have criminal records and complex problems. Follow-ups of up to 9 years continue to show differences between MTFC groups and control groups, on important indicators of well-being in this high risk population. MTFC may not be as effective for young people in care with complex emotional problems and may not be as effective in countries outside the US.

\section{MTFC for younger children}

A derivative of MTFC has been developed for school aged children, called Middle School Success, which has both a foster parent and a youth component, focusing on skill building. This intervention appears to have been evaluated in one study, which was described in two papers (Kim \& Leve, 2011; Smith, Leve, \& Chamberlain, 2011). This study showed that children who completed the intervention had significantly decreased internalising and externalising problems at 6 month follow-up and an increase in pro-social behaviour and fewer placement changes at 12 month follow-up. At three years after the intervention, the youth had less substance use, compared to controls.

MFTC-P for pre-schoolers has been extensively researched. The programme is also known as Early Intervention Foster Care and is a downward extension of the adolescent programme to pre-schoolers. The intervention uses a team approach with foster parents receiving extensive training, consultation and support. Children have a behavioural specialist and attend a therapeutic play group. Follow-up carers are offered family therapy to generalise the gains.

There have been several randomised control trials of this programme with significant increases in secure behaviour and significant decreases in avoidant behaviour and changes in cortisol levels comparable to children who have never been in foster care (Fisher \& Kim, 2007, 2009; Fisher, Kim, \& Pears, 2009; Fisher \& Stoolmiller, 2008; Fisher, Stoolmiller, Gunnar, \& Burraston, 2007). The intervention also improved children's changes of attaining a permanent placement. In a recent study, neurobiological findings with evoked response potentials showed that children who participated in the intervention were more responsive to 
external feedback than controls and were more similar to community children than foster children in the control group (Bruce, Fisher, Pears, \& Levine, 2009).

Laurent, Gilliam, Bruce, and Fisher (2014) also found that the MTFC-P intervention normalised cortisol patterns over the day and found that the link between abnormal cortisol patterns and anxiety was reduced in the MTFC-P group. These results were found over a 6 year follow-up, indicating MTFC-P had long lasting effects for those children. The net benefits of procuring permanent placements were found to outweigh the extra costs of providing MTFC-P, although the results were only significant for the group of prescoolers with placement instability (Lynch, Dickerson, Saldana, \& Fisher, 2014).

In summary, MTFC for younger children, and particularly pre-schoolers, have shown that children who take part in MTFC are more similar in their neurobiology to community children than to foster children who did not take part in the intervention. The finding that these effects endured 6 years later is a strong indicator of the effectiveness of the programme and the ability to change a fundamental aspect of foster children's functioning. However, this research has relied on measures of daily problem behaviour rather than mental health screening so the effects for children's mental health have not been established.

\section{Interventions based on attachment theory}

Dozier and her colleagues' Attachment and Bio-behavioural Catch-up (ABC) is the most rigourously evaluated attachment theory based intervention to be used with foster children. The programme aims to increase parental sensitivity and responsiveness and consists of 10 weekly sessions with intervention targets such as following the child's lead, reinterpreting the child's rejecting behaviours and increasing nurturing such as touching and cuddling the child. The parents of control group children complete a Development and Education for Families programme to control for the hours of intervention received.

Children in the intervention groups show more typical cortisol patterns, resembling those of children who have never been in care (Dozier, Peloso, Lewis, Laurenceau, \& Levine, 2008; Dozier et al., 2006). Dozier et al. (2006, 2008) argue that the ABC intervention can enhance children's regulatory capacity and change children's biology so that they more closely resemble typically developing children. Dozier, Lindhiem, Lewis, Bernard, and Peloso (2009) found that children in the intervention group showed less avoidant behaviour and were more able to seek care from the foster parent when distressed. A later study with 
parents at risk of maltreating their child found a significant increase in the children's secure attachment and a decrease in disorganised attachment (Bernard et al., 2012).

Findings for behaviour problems have been more mixed. Dozier et al. (2006) reported fewer daily problem behaviours for older but not younger toddlers after the $\mathrm{ABC}$ intervention, using the Parent Daily Report Checklist (PDR). Sprang (2009) found foster children's internalising and externalising behaviour on the Child Behaviour Checklist (CBCL) significantly decreased after the ABC intervention with 29 foster parents, compared to those on a waitlist. The best predictor of these decreases was parent engagement with the intervention.

Recent studies have evaluated $\mathrm{ABC}$ outcomes up to six years after the programme was delivered. Lewis-Morrarty, Dozier, Bernard, Terracciano and Moore (2012) showed that children who had received the $\mathrm{ABC}$ intervention some four years earlier had higher cognitive flexibility and theory of mind skills than foster children who received the control intervention and they were indistinguishable from the non-fostered children. Lind, Bernard, Ross and Dozier (2014) reported that children in the ABC intervention showed lower levels of negative affect expression and less anger towards their parents during a challenging task and in general, compared to the control group.

Few other attachment interventions have been researched with foster children. An individualised programme, based on attachment theory, found that, more children were adopted and fewer children who returned to their birth parents were maltreated (Zeanah et al., 2001). An intervention focussing on mothers' sensitive responding with adopted infants using video feedback, found that mothers' sensitive responses were enhanced and the number of children with disorganised attachment was decreased, compared to control groups (Juffer, Bakermans-Kranenburg, \& van IJzendoorn, 2005; Stams, Juffer, van IJzendoorn, \& Hockensbergen, 2001).

In summary, attachment interventions involving the foster parent and the foster child, have shown promising results. There is a growing evidence base that the $\mathrm{ABC}$ intervention has effects on children's attachment security, behaviour problems, neurobiological functioning, executive functioning and negative affect. However, only one study has been independent, and further studies using mental health questionnaires are required.

\section{Psycho-education groups targeting attachment}

In the past ten years, a number of training programmes have been developed to assist foster parents to understand attachment theory and the nurturing and support that foster 
children need. These programmes are group based and largely didactic or psychoeducational. The research on these programmes is methodologically weak. A recent review highlighted the lack of rigorous studies of these training programmes (Kerr \& Cossar, 2014). A series of small, pilot studies of the Fostering Attachments training programme, found little evidence of effectiveness (e.g., Golding \& Picken, 2004; Laybourne, Andersen, \& Sands, 2008). A further study using this intervention is outlined in the section below (GurneySmith, Granger, Randle, \& Fletcher, 2010).

In summary, psycho-education programmes may not be as effective as therapeutic interventions, which include both the parent and child. However, these interventions have been conducted with the foster parents of older children, compared to the therapeutic interventions, in which almost all participants were under the age of three years.

\section{Interventions targeting mind-mindedness}

Two small preliminary studies have been conducted with interventions targeting foster parent mind-mindedness. A study using the Fostering Attachments training programme noted above, with five foster parents and 10 adoptive parents, found no significant difference in mind-mindedness, although there was a significant increase in parents' mind-related comments about their own reactions to a rupture in the parent-child relationship (GurneySmith et al., 2010). Significant decreases in SDQ total scores at post-intervention were not maintained at follow-up. The study had no control group and mind-mindedness was measured by the total number of mind-related comments, rather than the usual proportion of mind-related comments. The authors raise a paradox, which is notable in many foster care interventions, that is, "the groups are rated highly by participants but change in the child's behaviours is inconsistent" (Gurney-Smith et al., 2010, p. 51).

An intervention based on mind-mindedness theory was delivered to 20 Dutch adoptive families, with the aim of increasing the child's mentalization, by helping the child to recognise their own and others' thoughts and feelings (Colonnesi et al., 2012). The intervention taught parents to "name" the "behaviors, feelings, wishes, intentions, and thoughts of the child according to a set of well-specified criteria" (p. 181). The intervention also used video feedback to promote parental sensitivity. Surprisingly, the study did not measure mind-mindedness. The study found limited improvement in some attachment ratings but not overall emotional and behaviour problems. No control group was used. 
In summary, mind-mindedness has only been used in one small pilot study, with limited results and a study evaluating teaching mind-mindedness skills did not include a measure of it.

\section{Meta-analyses and reviews}

Few meta-analyses have been conducted for foster care interventions, due to the heterogenous natuure of both the interventions and the research methodologies used (Leve et al., 2012). A recent meta-analysis of interventions to help foster parents cope with the child's behaviour appeared to be poorly conducted, with the inclusion of studies of the general population and a case study in a meta-analysis of foster parent interventions (Andel, Grietens, Strijker, Van der Gaag, \& Knorth, 2014). Some reviews have come to strikingly different conclusions. For example, in two systematic reviews using similar study quality criteria, Everson-Hock et al. (2012) found only 6 studies which met their inclusion criteria, having excluded treatment foster care, while Kinsey and Schlösser (2012) found 30 studies which met their criteria and they found that the majority of studies were of high quality. Another review also concluded the studies were of poor quality (Rork \& McNeil, 2011).

Considering recent reviews together suggests that there is very little evidence for the widely used foster care training packages such as MAPP and PRIDE in the US and that the most effective interventions are parent training programmes specifically developed for foster parents, interventions working with both foster parent and child based on attachment theory and MTFC and its derivatives. There is widespread agreement that little attention has been paid to complex problems and trauma (Ai et al., 2013; Dorsey et al., 2008; Kinsey \& Schlösser, 2012; Leve et al., 2012).

Kinsey and Schlösser (2012) found 30 studies of 20 different interventions in their systematic review of interventions for foster children, that most interventions were with foster parents only and most studies were of high quality. They found that researchers studying three interventions, KEEP, MTFC and ABC, each published multiple papers on a single randomised control trial. Dorsey et al. (2008) also found 30 studies and noted they were very diverse, that 11 of the 30 were written between 1979 and 1983 and that there were mixed results for child behaviour problems. They suggest that foster parent training should have two levels: firstly, foster care orientation and child safety packages and secondly, skills based programmes. 
Leve et al. (2012) reviewed randomised control studies of interventions which improved at least one outcome and had at least 15 participants per group. They noted that some interventions which have been shown to be effective for preventing maltreatment, most notably Triple P, Parent-Child Interaction Therapy and Nurse-family Partnership programmes, have not been tested using a randomised controlled trial with foster children and that standard parent interventions may not be sufficient to produce change in this population. They found only eight interventions that met their inclusion criteria and four of these originated from the Oregon Social Learning Centre (see above). The other interventions were Attachment and Bio-behavioural Catch-up, the Incredible Years Programme, the Bucharest Early Intervention Project, and the Fostering Individualised Assistance Programme (Leve et al., 2012).

Leve et al. (2012) concluded that "Overall, effective programs are attachment focused or have evolved from parenting interventions based on social-learning frameworks" (p. 10). The authors noted several limitations to the research including the lack of baseline data in some studies, lack of long term follow-up data on some interventions, little testing outside the US and problems with the size, robustness and generalisability of findings. A considerable problem noted by both Leve et al. and Kinsey and Schlösser (2012) is the variable number of publications reporting on a single sample, sometimes making it difficult to assess how many studies have been carried out. Kerr and Cossar (2014) note that behavioural and mental health outcomes have not been reported in a number of papers.

\section{Summary}

Although original research was not promising and some widely used foster parent training packages have little empirical evidence, there is now a strong body of literature that suggests that specifically developed parent training programmes, interventions based on attachment theory, which include the foster parent and child, and wrap around services are effective in assisting foster parents and children. However, some of these interventions have only been used with very young children and the intensive programmes are expensive to implement, although they have been found to be cost effective (Lynch et al., 2014).

It is possible that the complexity and severity of foster children's difficulties (TarrenSweeney, 2013) make it more difficult to bring about positive and clinically significant change, especially in behaviour problems and for adolescents without criminal histories. Two studies have shown almost no statistically significant change on any measure (Macdonald \& Turner, 2005, Minnis et al., 2001) and other interventions show less change 
than expected in established interventions such as the Incredible Years Programme (Linares et al., 2006). There is little support in the research for the effectiveness of brief psychoeducation programmes based on attachment theory and this may reflect their group rather than individualised focus (Kinsey \& Schlösser, 2012). Interventions with foster children and youth may be less effective in other contexts or countries than the one they were developed in (e.g., Biehal, Ellison, \& Sinclair, 2011) or may show effectiveness on behavioural measures with only a subsample of children (e.g. Dozier et al., 2006).

Programmes which are comparatively inexpensive, and which assist school aged children as well as adolescents without offending problems, are required. The Lightbulb training programme described and evaluated in Chapter Seven, can be used with foster parents of children of any age, as there is an individual focus on one foster child, who is causing the foster parent the most concern, within a group format. The training programme uses the Relational Learning Framework to develop an understanding of the impact of adverse experiences on each particular foster child and an individualised treatment plan is developed for each child. Essentially, foster parents are shown how to access aspects of the child's internal working model, so that they understand the child's behaviour and can talk to the child about it in a way which enhances the child's understanding. 


\title{
Chapter Six-Helping foster parents understand the foster child's perspective: A relational learning framework for foster care ${ }^{1}$
}

\begin{abstract}
The behaviour of children in foster care is influenced by a variety of factors including previous experiences of maltreatment and adverse parenting, as well as the impact of separation from birth parents and placement in care. These factors make it difficult for foster parents to accurately interpret the child's behavioural cues, a necessary precursor to sensitive parenting. The relational learning framework introduced in this paper, drawing on attachment theory, facilitates the foster parent's access to some features of the child's mental representations, or internal working model, which may be pivotal in understanding the child's behaviour and therefore successfully managing it. Recent studies suggest that parent's ability to understand the child's psychological perspective, or mental state, is related to the child's cognitive and social development. This paper presents a method to enhance the foster parent's understanding of the child's psychological perspective. The model is currently being evaluated for use with foster parents, mental health and social work practitioners.
\end{abstract}

\section{Keywords}

Foster care, internal working model, mental representations, mind-mindedness, relational learning framework

\section{Introduction}

The relational learning framework, introduced in this paper, was developed as a method to help foster parents consider how their foster child's past experiences of maltreating and impermanent relationships may influence the child's ideas, expectations and behaviours in relationships. The framework is derived from attachment theory, and is compatible with the concept of core beliefs and interpersonal schemas, as articulated in Aaron Beck's cognitive theory (Beck, 1976), relating to the way in which adverse experiences affect children's views of themselves, others and the world. While the relational learning framework is based on attachment theory, it has been developed as a cognitive technique to aid foster parents and

\footnotetext{
${ }^{1}$ This chapter has been published as Kelly, W., \& Salmon, K. (2014). Helping foster parents understand the child in their care: A Relational Learning Framework. Clinical Child Psychology and Psychiatry, 19, 535547. doi:10.1177/1359104514524067 (with amendments for the style of the thesis).
} 
practitioners to understand the psychological perspective of the child. Thus rather than an attachment intervention aiming to improve foster parents' sensitivity and attunement, the primary aim of the relational learning framework is to remove barriers parents may have to understanding and empathising with their foster child.

The framework focuses attention on the child's mental representations, which are considered to be "the means through which children package their experiences and carry them forward' (Dweck \& London, 2004, p. 428). It integrates salient information from a child's history and experiences with information about their current behaviour, providing insight into the child's mental representations, ideas and expectations about him or herself and other people. This information can then be used by foster parents to provide the corrective experiences and verbal messages the child needs to develop more positive expectations of future relationships.

As it is presented in this paper, the relational learning framework can be used in case conceptualisation, formulation and case reviews. Using it with foster parents as an intervention requires further evaluation, which is in progress.

\section{Foster care and mental health}

Children placed in foster care show an array of complex behaviours influenced by adverse experiences such as maltreatment, witnessing violence, parental substance abuse and mental health concerns and impoverished and chaotic environments (Oswald, Heil, \& Goldbeck, 2010). The prevalence of clinically significant emotional and behavioural problems such as non-compliance, aggression, problems with impulsivity and attention, anxiety and depression in children involved in child welfare investigations has been found to be 42 to $48 \%$, with many of these children not receiving mental health care (Burns et al., 2004; Garland et al., 2001). Children in foster care show rates of externalizing behaviour over double that seen in children in the community, with an average of $42 \%$ for children in care, compared to 7 to $20 \%$ for children in the community (Keil \& Price, 2006). Factors related to foster children developing mental health problems include longer exposure to an adverse environment, coming into care at an older age, developmental delay, placement instability and lack of a permanent placement (Tarren-Sweeney, 2008a).There have been mixed findings on whether entry into foster care in itself has a positive, negative or neutral effect on children's externalizing behaviour problems (e.g., Berger, et al., 2009; Lawrence, et 
al., 2006) but children's behaviour problems have been shown to both contribute to, and subsequently increase after, the experience of placement disruption (Oosterman, et al., 2007).

Placement instability is common in foster care (Osborn, et al., 2008). In addition to effects on externalising behaviour problems, placement instability has negative effects on children's inhibitory control, trust in caregivers and sense of security and has been shown to increase indiscriminate friendliness or lack of reserve with unfamiliar adults (Pears, Bruce, Fisher, \& Kim, 2010; Unrau, et al., 2008). A minority of children do not achieve a stable placement, and some children with severe maltreatment histories and conduct disorder can experience up to 30 or more placement changes (Osborn et al., 2008). Once children start on a trajectory of placement disruption due to externalising behaviour problems it is likely to continue, with children who have two or more behaviour related placement disruptions having only a $5 \%$ chance of achieving placement stability two years later (Barber \& Delfabbro, 2004).

While little is known about how older children form attachment relationships in foster care, toddlers have been shown to have rejecting and avoidant behaviour in response to new caregivers and to take longer to develop stable patterns of attachment security than infants (Stovall-McClough \& Dozier, 2004). Some of these behaviours may interfere with the ability of foster carers to provide the child with the warm and responsive care needed to help overcome the effects of previous poor care (Bernier, et al., 2004). Despite this hurdle, young children in foster care become securely attached to their caregiver at very similar rates to biological parent-child relationships, with just over half developing secure attachment (Dozier, et al., 2001). However, young children in care have more difficulty adapting to their foster parent's parenting style when the parents themselves have an insecure state of mind regarding attachment and are more likely than biological children to develop a disorganized attachment (Dozier et al., 2001). This has been shown to be a risk factor in the development of mental health problems (Fearon et al., 2010).

The majority of children in foster care have been maltreated, with subsequent effects on their ability to regulate their emotions and stress responses (Kim \& Cicchetti, 2010; McCrory, De Brito, \& Viding, 2010). Foster children show low basal levels of the stress hormone cortisol, and a flatter slope across the day, than low-risk children, although not as marked as children who remained with birth parents after a child protective services investigation (Bernard, Butzin-Dozier, Rittenhouse, \& Dozier, 2010). Foster children also have poorer visuo-spatial ability, language skills and general cognitive functioning, compared to other children living in low income but not maltreating families (Pears \& Fisher, 2005). 
Since children in foster care often do not receive any specialist mental health care (Burns et al., 2004) it is critical that foster care is as stable and reparative as possible.

\section{Treatment for children in foster care}

Most training programmes for foster parents are located within child protective services and focus on the decision to provide care and relevant policies and regulations. These programmes have been criticised for not paying sufficient attention to training parents in behaviour management (Dorsey et al., 2008). The authors note that 'there is remarkably little empirical evidence for the type of training that foster parents receive' (Dorsey et al., 2008, p. 1412) but that more recent studies have been more rigorous and have evaluated the use of interventions developed for other populations with foster parents. A Cochrane review found evidence across six randomised control trials that behavioural and cognitive behavioural programmes used with foster carers were not effective (Turner, et al., 2007) and a study of the use of outpatient mental health services for foster children showed little effect of these services for foster children (Bellamy, Gopalan, \& Traube, 2010).

A recent review located eight foster parent interventions which had an effect on at least one aspect of the foster child's functioning in a randomised control trial (Leve et al., 2012). These therapeutic approaches were Attachment and Bio-behavioural Catch-up which is an attachment-based intervention for pre-schoolers (Dozier, et al., 2008), behaviour management programmes for school aged children and the intensive Multi-dimensional Treatment Foster care used with pre-schoolers and adolescents (Fisher \& Kim, 2007). A systematic review found 30 studies involving 20 interventions and identified five types of interventions including wraparound services, relational and non-relational interventions for caregivers and children, caregiver training programmes and interventions for the child (Kinsey \& Schlösser, 2013). The study concluded that wraparound services and relational interventions were supported by empirical studies but that most caregiver training programmes were not.

It appears that these differing findings regarding the effectiveness of interventions in foster care may be a result of lack of rigour in early studies (Dorsey et al., 2008), methodological limitations (Rork \& McNeil, 2011), the degree to which programmes have tailored their content to the psychological factors thought to be critical in foster care and the adaptation of interventions shown to be effective with other populations (Leve et al., 2012). These authors note that 'Overall, effective programs are attachment focused or have evolved from parenting interventions, based on social learning frameworks' (p. 1206). 
A potentially fruitful target of intervention which has not been applied to the area of foster care is the foster parent's mental representations, or the way the foster parent conceptualises and thinks about the foster child's perspective and behaviour. Studies using the concepts of mentalisation and mind-mindedness in birth families have shown the impact of parent's mental representations on an array of socio-emotional outcomes for children (e.g. Meins, et al., 2001). Studies suggesting that these concepts may be usefully applied to foster care interventions are now addressed and a method to introduce this intofoster parent training and professional case conceptualisation is described.

\section{Mental representations and foster care}

Researchers have explored the influence of the way a parent thinks about their child through the concept of mind-mindedness and Meins (1997) has proposed that parents' levels of mind-mindedness may help to explain how attachment states of mind are transmitted from one generation to the next. This research has shown that parents who treat their young children as if they have a mind of their own, and who use a high level of mind-related comments in conversation with them, or verbal description of them, have children with secure attachment and an ability to reason about the beliefs and mental states of other people, referred to as theory of mind (e.g., Meins et al., 2001; Meins et al., 2003).

Fostering may affect the ability of the parent to interpret the child's mental state, or mind-mindedness, as Lawler (2008) notes that foster care relationships 'rely heavily on a foster mother's expectations and interpretations of a foster child's behavior, which usually includes an array of emotional deficits and behavioral problems' (p. 1131). Some authors have also noted the importance of foster carers not perceiving the child's difficult behaviour and need for affection as a threat, which may affect the parent's ability to care for the child in a non-defensive way (Wilson, 2006). It is possible that foster parenting makes higher demands on the parent's ability to take the child's perspective and to infer the child's mental state from their behaviour. Schofield and Beek (2005a) found that caregivers who were rated as highly sensitive had an ability to reflect and empathise with the child, believed they were central to the child's well-being and had developed theories about the child's past that helped them to explain their present behaviour, which impacted on the child's progress in the placement.

Children in care may also have mental representations or beliefs which reflect their adverse experiences and negative perceptions of future care. Milan and Pinderhughes (2000) 
found that children's negative perceptions or mental representations after maltreatment by their biological mother were related to the severity of maltreatment by the mother and influenced their new relationship with the foster mother. Studies with pre-school children have shown that maltreated children have more negative mental representations about the mother-child relationship than do non-maltreated children and view such relationships as 'less fulfilling, safe and reliable' (Stronach et al., 2011, p. 142). In one study, over half of the children who had been in a stable placement since toddlerhood had negative self perceptions at the age of 5 and 6 years (Ackerman \& Dozier, 2005). These were more likely to develop when foster parents were not fully emotionally invested in their foster children (Ackerman \& Dozier, 2005).

Attachment theory proposes that the above findings can be explained by the concept of the child's internal working model, meaning that, over time, children build a representational model of themselves and their caregiver. Bowlby proposed that 'each individual builds working models of the world and of himself in it, with the aid of which he perceives events, forecast the future, and constructs his plans' (Bowlby, 1973, p. 203). The idea of the internal working model is inherently a relational one and parents are also proposed to have an internal working model of the child and of the self as an attachment figure (Bowlby, 1973).

Furthermore, the relationship between parent and child is influenced by both individual's internal working model and change in one person's behaviour may bring about resistance in the other and an attempt to return the relationship to old patterns (Bretherton \& Munholland, 2008).

A recent study of the causal attributions foster parents, residential caregivers, social workers and mental health professionals make when dealing with foster children's challenging behaviours showed that individuals have diverse views on the origins of and solutions to the challenging behaviour of children in care (McLean, Kettler, Delfabbro, \& Riggs, 2012). Explanations of behaviour were categorised into six themes including conceptualising behaviour as learnt, as carrying meaning and purpose, as a conscious choice or as arising from constant change in the child's life, from strong emotions and from the child's attachment history. The authors describe some of the implications of these diverse frameworks for who may be blamed for the behaviour, whether it is seen as amenable to help, and for the ability of workers with different views to collaborate (McLean et al., 2012). The relational learning framework, developed by the first author, uses the concept of the child's internal working model to provide a way to integrate the various explanations foster parents and professionals have for foster children's behavioural difficulties. 


\section{The Relational Learning Framework}

The relational learning framework can be used to assist foster parents to understand how the child's behaviour provides crucial clues about how their previous adverse experiences have changed their view of themselves and others. For example, two siblings may have experienced similar physical abuse with very different outcomes: one sibling may be aggressive and the other may be withdrawn. These two different outcomes may be indicators of each child's mental representations - one involving notions of power and dominance and one involving notions of further hurt and fear. If foster parents are taught to think about the child's internal working model and how that influences the child's behaviour, it is possible that this may change the foster parent's attributions regarding difficult behaviour, particularly blame of self or foster child, and their behaviour management of the child. This knowledge is important as children in care may have beliefs which impact on the effectiveness of reinforcement contingencies on behaviour. For example, a hug to a child without an abuse history may be positively reinforcing but to a child in care it may be aversive as it is perceived to be frighteningly intimate. Thus reinforcers may have different meanings for foster children which parents may need to take into account when using behaviour management techniques.

The child's insecurity and uncertainty about their future may influence their behaviour and mental health (Tarren-Sweeney, 2008a). A foster child who has been moved to several foster homes because of their behaviour may learn that increasing negative behaviour is a powerful strategy to influence their future. A child whose behaviour is driven by fear, such as a maltreated child who has experienced multiple placements and who fears being abandoned, asked to leave or abused, will need different behavioural responses from his or her foster parent than a child who does not have these fears. For example, a child may become very intrusive of the foster parent, checking emails and letters, going through the parent's bedroom drawers and questioning the parent. Typical behaviour management techniques such as locking up personal items and giving the child negative consequences for the behaviour may make the behaviour worse. The child's behaviour may be due to fear that the placement will end or that a letter will arrive with bad news about the child's future in it. In this case, the fear must be addressed first.

The relational learning framework can be seen as a way of increasing the foster parent's cognitive understanding of the child or mind-mindedness. Recent authors have noted the importance of assisting foster parents to understand the child in their care's behaviour, in the 
light of their past history (Wotherspoon, O’Neill-Laberge, \& Pirie, 2008). The aim of mental health consultation is to understand the experiences underlying the child's behaviour, which appears out of context and can seem inexplicable to the caregivers (Wotherspoon et al., 2008). This approach requires the mental health clinician to make an interpretation of the child's behaviour, in the light of their experiences, and then discuss this with the foster parents. The relational learning framework provides an alternative way to teach foster parents to think about the child in care, allowing them to make the connections themselves, with the therapist only highlighting information from theory and scientific knowledge, for example, the effect of maltreatment on brain functioning. This means that foster parents can apply the model to new situations as they emerge and to new children they may foster in the future.

The framework addresses the child's culture as studies have shown that children who are fostered by a family of a different culture report more internalizing problems and the foster parents report more externalizing behaviour problems (Anderson \& Linares, 2012). In a study of minority adolescents adopted by White parents, Leslie, Smith, and Hrapczynski (2013) found that discrimination had a negative impact on the youth but that this was less stressful if their adoptive parents 'worked to promote cultural pride and prepared them for dealing with racism' (Leslie et al, 2013, p. 78). Clinical experience of the first author shows that children may reject their own culture if they perceive the culture to be inseparable from the maltreatment they have experienced within their birth family. It is thus important to examine what attributions the child has made about their culture and if their foster family is of a different culture, what attributions have been made about this difference. The relational learning framework is currently being evaluated with groups of foster parent and foster care practitioners.

\section{Applying the Relational Learning Framework}

The framework is a work in progress. It has been used over 200 times in clinical consultations and is currently being evaluated. It can be used as part of clinical supervision, in multi-disciplinary team meetings and for intake purposes. It takes 1-2 hours to complete and then can be reviewed at intervals to update the treatment plan and for tackling crises.

The relational learning framework is presented as a compact visual table (see Table 9) which can be used to consider all aspects of a particular foster child's experience and their implications for relationships. The purpose of the framework is to bring knowledge about the 
child together so that the team as a whole has the same information, to make connections between the child's past history and current experiences, to consider the meaning the child may have made of their experiences and to examine what the child's experiences and behaviour can tell us about their beliefs and mental representations. The ultimate aim is to form a treatment plan to gradually change the child's mental representations over time, emphasising what the child needs to learn through relationships, what the child needs to learn about emotions and how to talk to the child to verbalise both old and new representations, for example, 'You used to think everyone would hurt you but you have learned we don't hit here'.

The relational learning framework consists of five columns of information directly relating to a child's internal working model. The facilitator obtains the information from foster parents and foster care practitioners in the room and highlights important developmental and maltreatment factors (see Table 9). The reader will notice that the child is not involved in the process. The reason for this is that as it stands the method would be overwhelming for the child or young person and requires modification to ensure that it does not have a negative impact.

The framework is worked through according to the following steps.

Step 1: History and culture. The facilitator draws out the child's history starting with the first care and protection notification, what is known about the child's life prior to this notification and what occurred as a result of the notification. Emphasis is placed on each of the families the child has lived with, the quality of the care and both positive and negative experiences in each home and the number of changes the child has had. The facilitator draws the group's attention to the child's experiences which are likely to impact their behaviour and mental health, especially maltreatment, frightening and traumatic events, lack of basic necessities such as food, level of supervision, abandonment and changes of placement, school and neighbourhood. Lastly, the facilitator asks about the impact of these adverse events in the birth family on the child's perception of their own culture and whether maltreatment has changed that.

The role of the facilitator is to use scientific knowledge of child development and the effects of maltreatment to draw attention to formative experiences. The facilitator discusses the impact of events happening at a particular developmental level and what sense the child may have made of the event, given the expected cognitive and language ability at that age. Connections are made between events in the child's life and their behaviour, such as a child who was starved hoarding food in their bedroom. The facilitator focuses on the meaning or 
attributions the child may make about events so close attention is paid to any times the child was singled out or treated differently from siblings, especially if siblings were returned home but the child remained in foster care, and any events the child may take blame upon themselves for, such as a child having to move placement because a foster parent became ill. This information draws attention to the factors which may be affecting the child's current behaviour and mental health.

Step 2: Problem behaviours and strengths. In this section of the framework the issues the child is struggling with at home, school and in the community are highlighted. The child's strengths are also covered as they can provide information about what aspects of the child's life have not been affected by their experiences.

Step 3 and 4: View of self and the world. The foster parents and social workers are invited to consider what the child's experiences and set of problems/strengths suggest about the child's beliefs about him or herself. Any suggestions are written up in the first person such as 'I'm not loveable'; 'I'm the odd one out'; 'I have to take care of myself'. They are then invited to consider what the child's experiences and set of problems/strengths suggest about their beliefs about other people or the world such as 'The world is unsafe'; 'People leave you'; 'Relationships don't last'. Conversely, a child's pro-social behaviour may suggest the child believes that people and relationships are worth persevering with.

The participants are then asked to consider what this history is likely to have made a child conclude about families, relationships, leave takings, women and men and how you solve problems. The participants are prompted by questions such as 'What would the child think a family is...Men are...women are...' This technique brings out important child representations such as 'Women can be treated however you like'; 'Families don't last'; 'If you have a problem, do something so you get to leave'. These representations can have a major impact on the child's behaviour and need to be changed through a myriad of experiences which negate conclusions formed in an abusive family environment.

Step 5 Emotional and relationship needs. Having completed the sections of the framework outlined above, it becomes clear what corrective experiences the child needs. Participants usually say these in global terms e.g. security or permanence. They can then be asked 'What is it about security that the child needs to experience? What would they learn from permanency?' By answering these questions, foster parents begin to clearly see what aspects of care the child would particularly benefit from. 
Step 6: Putting it into words. Foster parents are then taught how to frame up the ideas they want a child to learn and to talk to the child about it. For example, a 10 year old child who has had supervisory neglect and been allowed to roam the neighbourhood, come home at any time and often found the house unoccupied may have difficulty adjusting to family life and appropriate supervision. The group may decide the child would find it hard to keep to rules and may find ordinary family life confining. The foster parent is encouraged to help the child understand his predicament. The parent might say 'No wonder you find it hard when I tell you to be home after school, you used to go wherever you wanted to. Families need a rule like that to keep you safe. We have the rule because we care what happens to you'.

\section{Case example}

This case example is a composite of several real case histories. Floyd is a 13 year old boy who was placed in residential care after he could not be contained in a foster family setting. In foster care, he absconded for more time than he was actually present, he was brought home several times by police in an intoxicated state and he was being recruited by a local street gang. He had committed several burglaries, including of his grandfather and other family member's homes. Prior to coming to the attention of child protection services, Floyd had experienced much disrupted care. Until the age of 4 years, he had been in the care of his parents, whose drug taking meant he was not fed regularly, was often left unsupervised and he had witnessed violence and intimidation of his parents due to drug debts. At the age of 4 years, 6 months, Floyd went to live with his maternal aunt but his behaviour was too difficult for her to manage. His grandfather could manage his behaviour but he did this by not setting limits on Floyd and allowing him to do what he wanted. Because of these difficulties with his behaviour, several family members took turns looking after Floyd. When his behaviour worsened, another family member would look after him for a while. Floyd's father is serving a five year jail sentence for assaulting Floyd's mother, which Floyd witnessed. Because the family had a strong belief in avoiding involving professionals in their lives, a first notification to child protection services did not occur until Floyd was 11 years old. Floyd was placed with an interim carer and had two prospective permanent placements, which both broke down due to his absconding. He fared better with his maternal grandmother for a period of a year but became increasingly threatening towards her, which culminated in an incident where he threatened her with a knife. He was then placed in a residential setting. 
Step 1: History and experiences. The aim of doing the relational learning framework for Floyd is to consider what his experience and behaviour can tell us about his mental representations. The salient factors for Floyd include neglect by his parents, witnessing violence, the family passing him around different family members when his behaviour became unmanageable, the family culture of not seeking help or alerting authorities, the number of changes of caregiver and his reaction to being placed (running away, violence). The group is encouraged to consider the developmental impact of Floyd being hungry and unsupervised as a toddler, being moved for difficult behaviour, having no help or involvement of social services until Floyd was 11 years old and witnessing frightening events when he was too young to influence them. The group is invited to think about what Floyd may have made of these events and what conclusions he may have come to about himself and his relationships. The facilitator might summarise Floyd's experiences as follows: 'Floyd was with his mother, then his Aunt, then his grandfather, then moved around their care for short periods, and then had 3 placements with foster families before moving to his grandmother's and then a residence. That amounts to 7 major changes of caregiver and several periods of unstable care where he moved around every 2 or 3 weeks. What would he learn from that?' Summaries such as this highlight the impact of his family and foster carer's not managing his behaviours and can be discussed to highlight conclusions he may have drawn from these experiences, such as that he is more powerful than adults and that the way to solve a problem is to leave the situation.

Step 2: Problem behaviours and strengths. The pattern of Floyd's behavioural strengths and difficulties can inform us about his possible representations of relationships. Floyd's use of absconding, threatening violence and gang associations suggest that he has learned to value power and control over others and that violence can be an effective strategy for him. One of his strengths is a close relationship with his grandfather, suggesting that Floyd may not have given up on the possibility of getting close to people.

Step 3 and 4: View of self and the world. Given Floyd's history and behaviour, the group is asked to consider what Floyd might think about himself and the meaning he may have attributed to events, e.g. Floyd may have concluded that he was moved between family members' homes when his behaviour escalated because he is too dangerous to be in a family and that no one can control him. The group then considers what Floyd's experiences and behaviour tells them about his view of other people and the world such as that adults are not effective and can't be relied on to keep you safe and that violence is the way to solve problems. 
Step 5 and 6: Treatment plan and verbal messages. When participants are asked what a child in foster care needs from relationships they will invariably say love, care, stability, safety and someone to believe in him, such as a mentor. These generalised qualities are deconstructed to examine what Floyd needs to know or understand about each experience e.g. what is it about stability that he needs to learn; what does he need to understand about relationships? Answers may include learning that families can be together without fighting, that relationships can last and that he can be helped to manage his behaviour. The group then comes up with brief messages which can be said to Floyd to assist him to change his negative beliefs e.g. 'We don't have to fight'; 'We can solve this'; 'You don't have to leave to feel ok'; 'Feeling bad doesn't need to spoil us having a nice time' and so on. The aim of these verbal messages is to reinforce the differences in the relationships in the foster home, compared to the child's previous adverse environment, that the child may not have attended to or understood. Putting these differences into words helps to gradually change the child's mental representations and understanding of both self and relationships.

\section{Conclusion}

There is preliminary evidence that foster parents' thinking about the child in their care influences the outcome for the child (Bernier \& Dozier, 2003; Schofield \& Beek, 2005a). There is also evidence that children's thinking and negative mental representations affects their relationship with new foster parents (Milan \& Pinderhughes, 2000). The relational learning framework offers a window into the child's internal working model and mental representations which may modify the foster parents own mental representations, such as increasing understanding of the origins of the child's behaviour and decreasing self-blame.

The framework can be used to assist foster parents to make sense of what is happening for the child and within the family. It also allows foster parents and social workers to quickly develop a shared view of the young person and their difficulties and can be used to sort out divisions within the caretaking team. Two studies are currently underway to evaluate the effectiveness of using the framework with foster parents, social workers and mental health clinicians who work in foster and residential care. 
Table 9 The Relational Learning Framework

\begin{tabular}{|c|c|c|c|c|}
\hline $\begin{array}{l}\text { Child's history \& } \\
\text { culture }\end{array}$ & $\begin{array}{l}\text { Child's problems } \\
\text { and strengths }\end{array}$ & View of self & $\begin{array}{l}\text { View of } \\
\text { others/world }\end{array}$ & Needs/Plan \\
\hline $\begin{array}{l}\text { Reasons for first } \\
\text { child protection } \\
\text { notification. } \\
\text { Placement history. } \\
\text { Quality of care with } \\
\text { birth parents and in } \\
\text { each placement. } \\
\text { Adverse experiences } \\
\text { e.g. trauma, } \\
\text { abandonment, } \\
\text { violence. } \\
\text { Culture of child and } \\
\text { foster parents. } \\
\text { Child's view of their } \\
\text { culture. }\end{array}$ & $\begin{array}{l}\text { What behavioural } \\
\text { and emotional } \\
\text { difficulties does the } \\
\text { child have? } \\
\text { What is the child } \\
\text { struggling with at } \\
\text { home, school and } \\
\text { with peers? }\end{array}$ & $\begin{array}{l}\text { What would the } \\
\text { child's experiences } \\
\text { lead them to } \\
\text { conclude about him } \\
\text { or herself? } \\
\text { Turn general words } \\
\text { like lonely into I } \\
\text { statement e.g. "I } \\
\text { feel alone". } \\
\text { What would the } \\
\text { child make of their } \\
\text { experiences? } \\
\text { How would they } \\
\text { make him or her } \\
\text { feel? }\end{array}$ & $\begin{array}{l}\text { What would these } \\
\text { experiences make } \\
\text { the child think } \\
\text { about other people } \\
\text { and the world? } \\
\text { What would the } \\
\text { child think about } \\
\text { safety, families, } \\
\text { whether } \\
\text { relationships last } \\
\text { and what happens } \\
\text { when things go } \\
\text { wrong? }\end{array}$ & $\begin{array}{l}\text { What does the child } \\
\text { need to learn about } \\
\text { relationships? } \\
\text { How can this be put } \\
\text { into simple words } \\
\text { for the child? } \\
\text { Think about the } \\
\text { conclusions child } \\
\text { may have made and } \\
\text { how to address } \\
\text { these e.g. "You } \\
\text { don't have to break } \\
\text { rules to be noticed". }\end{array}$ \\
\hline
\end{tabular}




\section{Chapter Seven-Study 2: A multiple-baseline study of the effectiveness of a training programme for foster parents}

\section{Introduction}

Although there are no published reports of foster parent mind-mindedness in the literature, the results of study one suggest that parent mind-mindedness and attachment perceptions may be worthwhile targets to incorporate in foster parent training. As this intervention study was conducted concurrently with Study one for practical reasons, attachment perceptions are not targeted in this intervention and, indeed, this material would be difficult to target in a psycho-educational group of this kind. The main outcome variable in this study, therefore, is mind-mindedness.

Evidence-based interventions for foster care are required across the age range and for a range of presenting problems. There are only four interventions for children and youth in foster care which have robust empirical evidence for their effectiveness over several randomised control trials (see Chapter Four). All of these programmes have some limitations regarding their evidence base, age range, target problems and measures used. MTFC-A and MTFC - $\mathrm{P}$ are highly intensive wrap-around services which are expensive to implement but have been found to be cost effective (Lynch et al., 2014). The results of the adolescent programme in countries other than the US has been mixed (Biehal, Ellison, \& Sinclair, 2011; Westermark, Hansson, \& Vinnerljung, 2007). The other two programmes have been developed for the narrow age range of infants, toddlers and pre-schoolers (ABC and KEEP) and although there are several studies of these programmes they require replication from independent researchers (Toth et al., 2013). Thus it is important that other effective programmes which are cheaper to implement and cover a wide range of ages are developed.

The aim of this study is to investigate the effects of a training programme incorporating the Relational Learning Framework (RLF) described in Chapter Six. The RLF has been developed as a way to help foster parents understand the child in their care and mind-mindedness is the concept closest to operationalising this understanding. Mindmindedness is defined as the parent's ability to see their child as a separate person with their own thoughts, wishes and emotions. It is hypothesised that the RLF assists foster parents to see the child as an individual with a unique relational history which is influencing their current relationships. Only two pilot studies have investigated mind-mindedness as either a 
part of the intervention or as an outcome measure, both with significant limitations, including pre-post design and lack of control groups. Gurney-Smith et al. (2010) measured mindmindedness in their evaluation of the Fostering Attachments Group programme (Golding, 2007) based on attachment theory, and Colonnesi et al. (2012) used "naming skills" to increase mind-mindedness but did not measure mind-mindedness. The current study aims to take this work further by using an intervention which targets the foster parent understanding the child's perspective, a measure of mind-mindedness and a multiple-baseline design to allow some experimental control by comparing within subjects. This design has been recommended for pilot studies with small numbers (Blampied, 1999, 2001, 2013).

\section{Conceptual base of current intervention}

In response to the limitations of current measures of parent sensitivity as a predictor of children's security of attachment, researchers have investigated the impact of parental representations, or how parents think about their child, variously conceptualised as mentalization and reflective functioning (Sharp \& Fonagy, 2008) or mind-mindedness (Meins, 1997, 1998). Parental representations have been shown to be strongly related to parenting behaviour and child outcomes such as security of attachment and theory of mind (Rosenblum, McDonough, Sameroff, \& Muzik, 2008; Sharp \& Fonagy, 2008). Moreover, foster care research has found that foster parent's ability to take the child's perspective and understand their behaviour is related to successful outcomes for the child (Schofield \& Beek, 2005a).

The current study aims to evaluate a training programme designed to change parent mind-mindedness and attributions by increasing the parent's psychological awareness of the child in their care. The parent is taken through a framework which considers how a child's experiences and behaviour provide indicators about their view of self and others. This can be viewed as constructing a putative internal working model for the child, so that the relational effects of the child's prior experiences can be considered (see Chapter Six). Through understanding the impact of the child's past experiences and how they relate to the child's present behaviour problems, the parent is assisted to focus on the underlying causes of the child's behaviour, potentially increasing the accuracy of parental attributions about the child and appreciation of their mental state. Other research informing the programme includes the influence of parent sensitivity on foster children's outcomes, including attachment security (Altenhofen et al., 2013; Ponsiano, 2010) and the impact of maltreatment on children's emotion regulation (Luke \& Banerjee, 2013; Shipman et al., 2007). 
As it seemed unlikely that an intervention focusing on mental representations could impact on the foster child's behaviour problems on its own, further components were added to the intervention; namely behaviour management, emotional communication, parent stress management and problem solving and addressing the child's culture. Behaviour management was included as externalising behaviour has been found to be one of the enduring difficulties of children in care, remaining high after the child has been in care for some time (Lawrence et al., 2006; Wilson, 2006) and increasing after placement disruption (Oosterman et al., 2007). A module on emotional communication was added as most children in care have experienced maltreatment and are likely to come from homes with high levels of negative affect and low levels of emotional support (Shipman et. al., 2007). This experience is likely to result in children having poor emotional regulation and negative perceptions of both themselves and their birth parents (Milan \& Pinderhughes, 2000). Consequently, skills in emotion coaching were included in the training (Gottman \& Declaire, 1987). A stress and problem solving module was included as foster parenting is generally regarded as a highstress type of parenting and parents have a high burden of care (Murray et al., 2011).

The intervention was named the "Lightbulb group" as several foster parents and social workers had described their responses to the main component of the training (the Relational Learning Framework described in Chapter 6) as like "a light going on" and an "aha! moment".

There were six components to the intervention:

1. Psycho-education. The aim of the psycho-educational component is to assist foster parents to understand the impact of the child's early experiences on their response to a new parenting relationship, by completing the RLF for each child (see Chapter 6). The intervention also covers the need for children in foster care to have sensitive and nurturing care and the importance of the foster parent providing a secure base for the child to rely on when emotionally distressed;

2. Mind-mindedness. The aim is to increase the foster parent's focus on the foster child's emotions and mental states (mind-mindedness) by increasing their awareness of the child's emotional and psychological perspective, especially during episodes of difficult behaviour;

3. Emotional communication. The aim is to assist foster parents to develop skills in conveying empathy and understanding to the child and talking about everyday experiences and emotions, as well as past experiences if appropriate; 
4. Behaviour management. Behavioural techniques were taught by covering basic social learning principles and teaching the foster parents to do a simple functional analysis, relating to the special circumstances of foster children. This analysis includes a) Why is the behaviour happening now (triggers)? b) What is the child trying to achieve through the behaviour e.g., to push the foster parent away? c) What emotion is the child trying to change through the behaviour? d) What is the meaning of the behaviour e.g., the child may steal food as they fear there won't be enough for them;

5. Problem solving and stress management. Each session included modelling of problem solving skills by using them when a foster parent asked a question about their child's behaviour and foster parents were encouraged to use these skills at home. A brief stress management module included a definition of stress, a simple stress audit and lifestyle changes the foster parents would like to make, a relaxation and mindfulness exercise and identifying unhelpful thoughts when dealing with difficult behaviour.

6. Culture. Research has shown that foster children and youth have better outcomes if they are placed with parents of the same culture and that youth find it hard when fostered in a family of a different culture (White et al., 2007). They also do better when foster parents of a different culture value the child's culture and assist the child to deal with racism and discrimination (Leslie, et al., 2013). This module introduced these ideas and considered ways the foster parent could value the child's culture. 


\section{Study 2 (a) Pilot Group}

\section{Method}

\section{Rationale for multiple-baseline research design}

Most studies of treatment outcome in clinical psychology use between group designs, with random allocation to the groups, in order to "...rule out threats to internal validity and alternative conceptual interpretations of the findings by including various control groups in the design" (Kazdin, 1980, p. 122). The randomised controlled trial is seen as the gold standard for outcome research, to the extent that bodies such as the Cochrane library, which review psychological treatments, generally exclude all other types of study. However, at the development stage of an intervention, a randomised controlled trial may not be a suitable research method to use, as the rigour and cost cannot be justified by any preliminary data. While many studies use a pre-post design in these circumstances, single case designs have been recommended as the participant acts as their own control and the comparison is between different periods of control and treatment conditions (Blampied, 1999, 2001, 2013; Kazdin, 1980). A widely used single subject design is the reversal or ABAB design where a treatment is applied and withdrawn once or more (Barlow, Nock, \& Hersen, 2009), however, there are circumstances where this approach may not be possible or ethical and the most common alternative is the multiple-baseline design (Barlow et al., 2009). The use of multiplebaselines is designed to serve as an experimental control, to lessen the likelihood that intervention effects could be due to other factors such as placebo effects (Kazdin, 1980).

Statistical techniques can also be applied to single case research. Parker, Vannest, and Davis (2011) outlined nine alternative methods and several papers have recently argued that the "percentage exceeding the median" method, that is, the percentage of data points in treatment phase B that exceed the median of the baseline phase A, is a useful summary statistic (Ma, 2006, 2009; Parker \& Hagan-Burke, 2007). The most common index used, "percentage of non-overlapping" data is seen as limited because if one or more data points hit a floor or ceiling level, then the score is zero, even though there could be a treatment effect (Ma, 2009). For this reason, the percentage exceeding the median technique has been recommended as a way of quantifying the magnitude of treatment-associated change.

Jacobson and Truax (1991) have argued that between group mean statistics do not provide information on the variability of the participants response to the intervention, the size and importance of the effect and whether it is clinically significant. Jacobson and Truax 
recommend the researcher calculates whether change is clinically significant, described as "The clinical significance of a treatment refers to its ability to meet standards of efficacy set by consumers, clinicians, and researchers"(p. 12). Jacobson and Truax also recommend that researchers use the Reliable Change Index to calculate whether change is statistically reliable, that is, outside the likely range of measurement error.

A final data analysis technique used in this study is the Modified Brinley

Plot (see Figure 7; Blampied, 2014), based on Jacobson and Truax (1991), Sobell, Sobell and Gavin, (1995) and Stunkard and Penick (1979). This is a scatterplot which shows the relationship between Time 1 data, plotted on the $\mathrm{X}$ axis, and Time 2 data plotted on the $\mathrm{Y}$ axis. The diagonal is the line of no change, where the score at Time 1 is the same as the score at Time 2, and visual inspection can be used to ascertain whether data points have moved in the desired direction. In addition, clinical cut off, measurement error lines and group means can be added to the graph (Blampied, 2013).

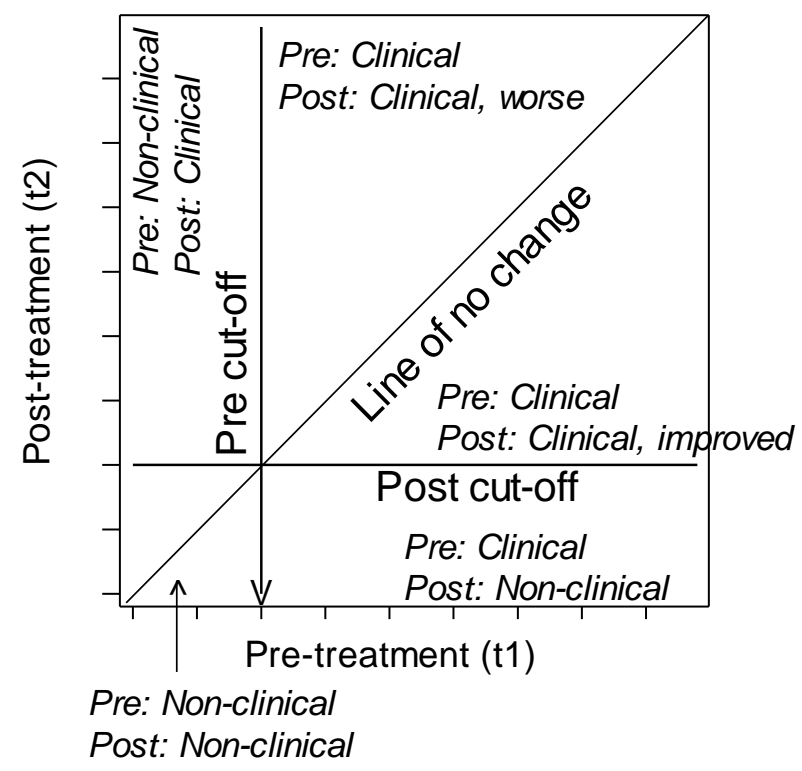

Figure 7. Modified Brinley Plot showing line of no change and pre- and post cut off areas for graph interpretation.

Note: Figure used with the permission of Nevile Blampied (personal communication, November 21, 2014).

\section{Research design}

There are three types of multiple-baseline designs: multiple-baselines across behaviours, across participants or across settings (Barlow et al., 2009). The current study uses a multiple-baseline across participants/cases design, which means that an intervention is 
delivered after differing lengths of baseline recording and visual inspection is used to assess the impact of the intervention, that is, change should occur in the treatment phase, and only in the treatment phase, regardless of baseline length. The baselines are non-concurrent, that is, the groups were delivered at different times. A multiple probe was also used in that two measures were completed weekly over both the baseline and treatment periods. The continuous measures were the number of child daily problem behaviours, parental stress related to these behaviours, and the child's overall functioning in a number of domains including family, school, interpersonally and overall. The repeated measures are described in the following section and were administered before the intervention began, in the last session of the intervention and at six month follow-up.

Foster parents took part in a training group and each group had a period of baseline recording of two, three or four weeks, which were assigned for practical reasons, such as the timing of the school holidays. The research was approved by the Victoria University Ethics Committee and the Ministry of Social Development Research Access Committee.

The training programme consisted of seven three hour sessions, scheduled two or three weeks apart, depending on the school holidays. A treatment manual was developed covering all of the above topics and participants were provided with a workbook. The content was delivered flexibly and each foster parent developed a plan for the intervening weeks.

The group sessions consisted of:

1) 30-60 minute check-in during which foster parents described how the placement was going, problems that had arisen and the group contributed to a problem solving discussion;

2) Education materials were presented and discussed on topics such as attachment relationships, providing sensitive care, the effects of abuse and loss, the importance of culture and behaviour management;

3) Learning new skills including the application of the RLF to each child, behaviour management, stress management and emotion coaching, using techniques developed by Gottman and Declaire (1997).

The research goal of the study was to examine the effctiveness of the training programme wth foster parents and whether the intervention was acceptable to the participants. The study hypotheses were that the training programme would increase foster parents' mind-mindedness and attributions about their foster child and that the intervention would have associated positive changes in foster child and foster parent well-being. 


\section{Participants}

Participants were four foster parents each with an adolescent aged between 12 and 15 years in their care (three boys and one girl). The foster parents were paid employees of a community organisation, which provides one-to-one foster care in families with no other dependants or foster children. The adolescents referred to the service have experienced multiple abuse and have been brought up in adverse environments characterised by neglect, lack of supervision, exposure to drug use and exposure to violence. The adolescents have often been placed in institutional settings and all have had multiple placements. The objective of the service is to stabilise the adolescent in care for a year and then transition them back to their family/whānau or to a permanent foster placement.

The caregivers were three women and one man, aged between 46 and 60 years. Two women were NZ European, one was of mixed ethnicity (unspecified) and the man was Māori. The women were living with a spouse and the man was parenting on his own. The training these foster parents had received ranged from none to several days and all foster parents took part in weekly meetings and attended group supervision with a therapist. They had 24 hour access to social work support. All had raised their own children to adulthood. The four adolescents had been in the care of the particular foster parent for between 3 and 12 months (see Table 10). Two of the adolescents were considered to have mild intellectual disability and one of these adolescents was also diagnosed with Attention Deficit Hyperactivity Disorder (ADHD).

Table 10 Demographic Information of Participants

\begin{tabular}{|c|c|c|c|c|c|c|}
\hline \multicolumn{3}{|c|}{ Foster parent } & \multicolumn{4}{|c|}{ Foster child } \\
\hline Ethnicity & Age & Gender & Ethnicity & Age & Gender & Months placed \\
\hline NZ Eur. & 60 & Female & NZ Eur. & 15 & Male & 6 \\
\hline NZ Eur. & 46 & Female & Māori/Eur. & 12 & Male & 6 \\
\hline Māori & 54 & Male & Māori & 15 & Male & 3 \\
\hline Unspecified & 51 & Female & Māori & 15 & Female & 12 \\
\hline
\end{tabular}

Participant 4 completed only one week of baseline measures, due to an administrative error. One participant did not complete the last session and this was conducted at a later stage by phone. Only one participant, whose child had not yet transitioned to a permanent placement or independence, completed the follow-up measures (not reported). 


\section{Measures}

Demographic questions. Background information was obtained from the foster parents via a phone interview, as described in Chapter Four. Questions included demographic information such as age and education level, and number of birth children and foster as well as questions about the child, including age at placement, length of time in placement etc. (see Appendix A).

Mind-mindedness interview ( Meins, Fernyhough, Fradley, \& Tuckey, 2001). As noted in Chapter Four, foster parent were asked one question "Can you describe [child] to me?" Following Bernier \& Dozier (2003), parents were also asked "What is he or she like?" The mind-mindedness measure has been shown to be stable over several months, has good construct validity and inter-rater reliability is consistently high (see Chapter Four for details). The interview was audio-recorded and transcribed verbatim by a research assistant. The interview was coded according to criteria set out in the Mind-mindedness coding manual, version 2.0 (Meins \& Fernyhough, 2010) and the coding process is outlined in Chapter Four. The kappa for mind-mindedness in the current study was $\kappa=0.85$ and the kappa for the valence of mind-mindedness was $\kappa=0.83$.

Parent Daily Report Checklist (PDR; Chamberlain \& Reid, 1987). This measure consists of a list of 30 behaviours and parents indicate whether the behaviour occurred on a specific day and whether it caused the foster parent any stress. The Parent Daily Report Checklist is usually administered by phone but in this study we used a written form which participants completed at pre- and post-intervention and six month follow-up for three days per week, and then twice a week over the duration of the study. This measure has been used with children aged from 5-12 years and shows good reliability with inter-call agreement of $97.6 \%$ and good stability over time ( $r=.82$; Chamberlain \& Reid, 1987). There was also good agreement between parents in 10 families on behaviours the mother saw as problematic. In terms of concurrent validity, Chamberlain and Reid (1987) found significant correlations between PDR scores and home observation data collected by independent observers. In addition, Chamberlain et al. (2006) found that daily scores of over six behaviour problems were associated with increased risk of placement disruption.

Parent Emotional Style Questionnaire (PESQ; Havighurst et al., 2010). Havighurst et al. (2010) created this 21 item interview questionnaire by adding seven items assessing parents' responses to their children's worries and fears to the Maternal Emotional Style Questionnaire (MESQ), developed by Lagacé-Séguin and Coplan, (2005). These 
questionnaires assess parents' responses to children's emotions, such as offering comfort to the child when experiencing emotion and talking to the child about emotional situations. The MESQ has been found to have two factors, namely emotion coaching and emotion dismissing and has moderate stability over a six month period. It has been shown to have significant correlations with parent goals in response to vignettes and to pre-schoolers behavioural regulation, as well as to the Meta-emotion Interview it is based on (Lagacé-Séguin \& Coplan, 2005).

Depression, Anxiety and Stress Scale (DASS; Lovibond \& Lovibond, 1995). As noted in Chapter Four, the DASS is a set of three self-report scales designed to measure the negative emotional states of depression, anxiety and stress. The DASS 21 had good internal consistencies, discriminant and convergent validity and has been shown to reliably measure changes in an individual's symptoms over time (see Chapter Four for details).

Parenting Sense of Competency Scale (PSOC; Johnson \& Mash, 1989). This 16 item scale has been found to have two factors, parent competency, including the ability to problem solve, and parenting satisfaction, including levels of frustration and motivation. Sanders et al. (2004) found an adequate level of internal consistency with alphas between .75 and .79. Good content validity and test-retest reliability have been found, and Johnson and Mash (1989) found significant negative correlations with the Child Behaviour Checklist.

Strengths and Difficulties Questionnaire (SDQ; Goodman \& Scott, 1999). As described in Chapter Four, this 25 item questionnaire covers positive and negative attributes with five scales including conduct problems, inattention-hyperactivity, emotional symptoms, peer problems and pro-social behaviour. This widely used measure has excellent reliability and validity (see Chapter Four for details).

Child-Parent Relationship Scale (CPRS; Pianta, 1994). This scale is completed by a parent and contains fifteen five point Likert scales designed to assess the adult's view of their relationship with the child. There are two subscales, the Closeness scale and the Conflict scale. The scales have been used in several large scale studies and internal consistency was found to be moderate, with good predictive validity with measures of emotion regulation and attachment security (Howes \& Ritchie, 1999; NICHD Early Child Care Research Network, 2004).

Attributions. Using Likert scales which were created for this study, foster parents were asked to describe an incident of their foster child's difficult behaviour and to what extent they attributed the behaviour to each of nine different possible causes: 1) the child's personality, 2) the situation at the time, 3) the child's past experiences, 4) the child's 
relationship with the foster parent, 5) the child being in foster care, 6) the child having been in several placements (if applicable), 7) the foster parent's parenting of the child, 8) the child's friends, and 9) the influence of the child's birth parents. Each Likert scale had a score from a minimum of one to a maximum of seven and scores were averaged for each individual across situations. The Likert scales were aimed at measuring parents' willingness to attribute the child's behaviour to a wide range of causes.

Treatment evaluation questionnaire. This questionnaire was developed for this study. Likert scales were used to evaluate the participants' satisfaction with the group. The ratings were between $1-5$ (minimum to maximum) for rating the usefulness of the group, whether it changed the participant's thinking, behaviour and interaction with the young person, the young person's response to this and whether they would recommend the group to a friend.

\section{Results}

The research goals of the study were to examine whether the intervention was acceptable to the participants, whether it increased foster parents' mind-mindedness about their foster child and whether the intervention had an associated effect on foster child and foster parent well-being. Firstly, participants gave feedback on their experience of the intervention, using an evaluation questionnaire created for this purpose. Secondly, results were considered at the group level using paired sample t-tests (pre- and post-intervention) and effect sizes (Cohen's d). Thirdly, the results were considered on an individual level using modified Brinley plots. The Reliable Change Index (RCI) was used when population norms were available and it is recommended that one or two of the most salient measures are chosen for this calculation (Barkley, Edwards, Laneri, Fletcher, \& Metevia, 2001). The measures chosen for which to calculate the RCI in this study were the Strengths and Difficulties Questionnaire, the Parent Daily Report Checklist and the Outcome Rating Scale. In addition, Clinically Significant Change or the clinical cut-off was used as an indicator of the clinical impact of the observed change. Finally, continuous measures were used to examine trends over time in relation to treatment phase changes.

Effect sizes were calculated using Cohen's d for paired designs using the formula recommended by Cummings (2012) which is which is $d=M_{\text {diff }} / s_{\mathrm{av}}$ where $M_{\text {diff }}$ is $M_{\text {pre }}-M_{\text {post }}$ and $s_{\mathrm{av}}=\sqrt{ } \mathrm{s}_{\text {pre }}^{2} s_{\text {post }}^{2} / 2$. The Reliable Change Index is a method of detecting change which exceeds the margin of measurement error using the formula $\mathrm{RCI}=\left(\mathrm{x}_{1}-\mathrm{x}_{2}\right) / s_{\text {diff }}$ where $\mathrm{x}_{1}$ is 
the participant's pre-test score, $\mathrm{x}_{2}$ is the participant's post-test score and $\mathrm{s}_{\mathrm{diff}}$ is the standard error of difference between the two test scores (Jacobson \& Truax, 1991). Clinically significant change is calculated by one of three methods for assessing if the post-test and follow-up means have moved away from the mean of the dysfunctional population or closer to the mean of the functional population. The method used for this calculation for each measure possible was the mid-way point between the sample mean at pre-intervention and the population mean (Jacobson \& Truax, 1991).

Treatment satisfaction. The group format was acceptable to the participants, with only one participant missing one session, which was subsequently made up. Likert scales were used to evaluate the participants' satisfaction with the group. All participants gave the maximum rating of 5 for the usefulness of the group. Participants rated the mean change in their thinking and behaviour and interaction with the child as $4.5(S D=0.58)$ where 1 is "not at all" and 5 is "a great deal". Responses for concomitant changes in the young person's behaviour received a lower mean rating of $3.75(S D=0.96)$. All participants used the maximum rating for recommending the group to others.

\section{Repeated measures}

The mean pre- and post- intervention scores for the group participants for pre- and post-intervention, paired samples t-tests (two tailed) and effect sizes are shown in Table 11.

Mind-mindedness and attributions. There was a significant increase in parents' mind-related comments or mind-mindedness from pre-intervention to post-intervention, with the mean score almost doubling from 0.20 to 0.39 , with a large effect size of 1.50 (see Table 11). Visual inspection of the modified Brinley plots (see Figure 8) shows that all participants increased in mind-mindedness. As there are no population norms for the mind-mindedness interview, the averaged mean of two community samples were used to calculate clinical significance. Meins et al. (2003) reported a mean of 0.44 and Lok and McMahon (2006) reported a mean of 0.36 and the mid-point between these means was used in this study $(0.40)$. This showed that all participants moved from the below average range to the mid-point of the two community samples' means.

Foster parents were asked to what extent they attributed an incident of their foster child's difficult behaviour to each of nine different possible causes (see method section for details). Mean attribution scores increased from 3.71 to 4.57 , which was not statistically significant. Visual examination of the modified Brinley plots shows that three of the four 
participants increased in their level of attributions made and the fourth participant had a high score at pre-intervention (a ceiling effect).
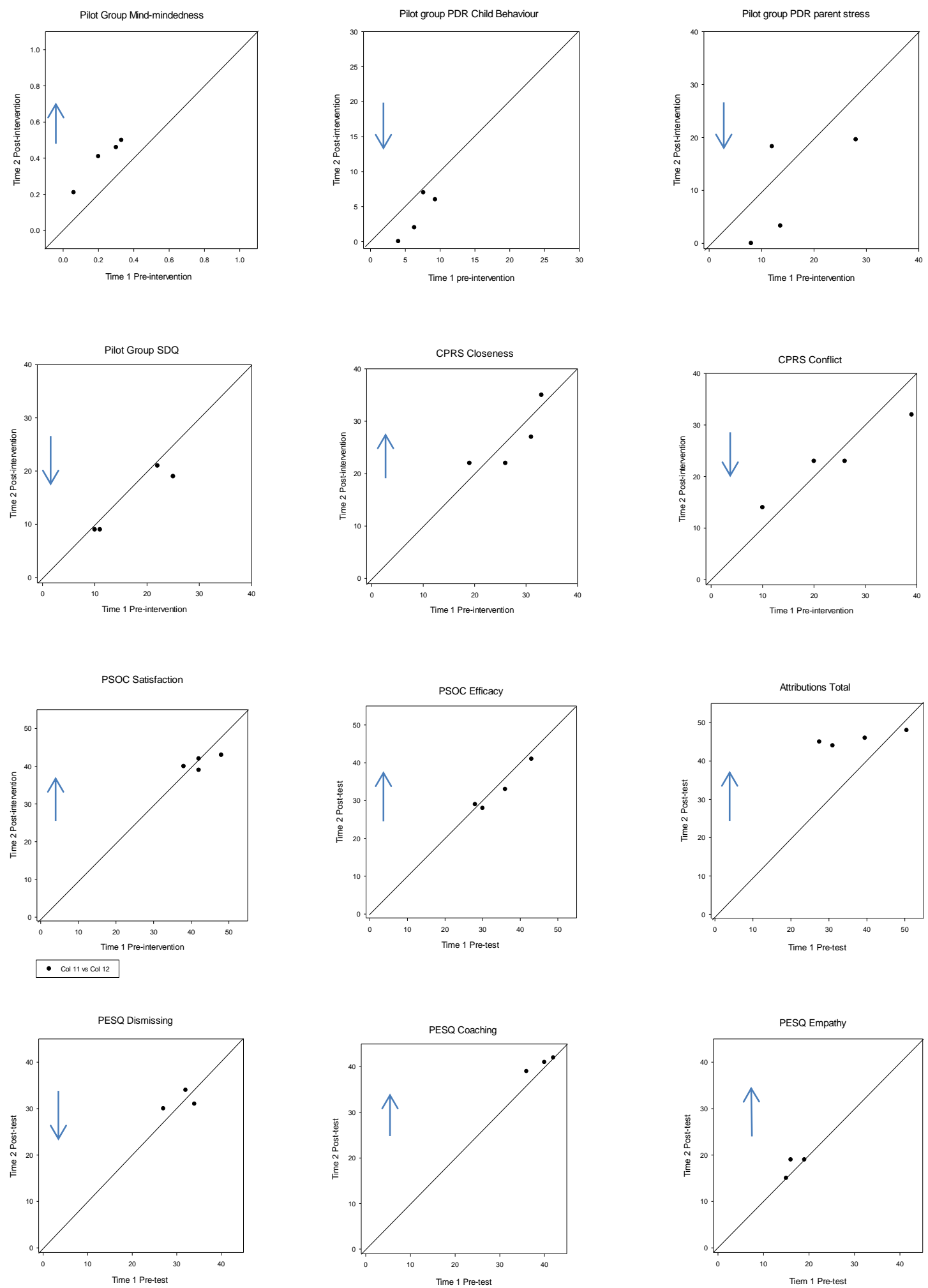


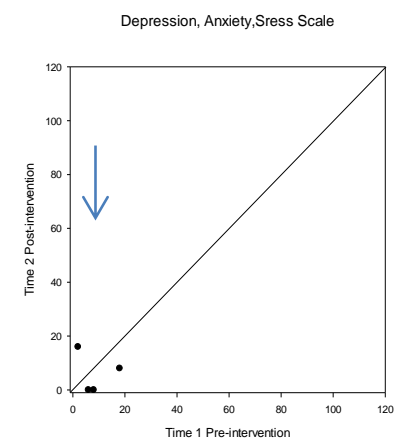

Figure 8. Modified Brinley plots showing pre-intervention scores on the $\mathrm{X}$ axis and post-intervention or follow-up scores on the $\mathrm{Y}$ axis for each measure and sub-scale.

Note. PDR $=$ Parent Daily Report, SDQ $=$ Strengths and Difficulties Questionnaire, CPRS $=$ Child Parent Relationship Scale, PSOC = Parent Sence of Competency Scale, PESQ = Parent Emotional Style Questionnaire and DASS = Depression, Anxiety and Stress Scale. Each point represents one participant. Arrows indicate direction of improvement.

Parent Daily Report. Daily child problem behaviours significantly decreased from a mean of 6.8 a day prior to the intervention to a mean of 3.8 at the conclusion of the intervention, with a large effect size (see Table 11). Figure 8 shows that all participants reported that the young person's daily problem behaviours had decreased. A cut-off of six daily problem behaviours has been regarded as at risk for placement breakdown (Chamberlain et al., 2006) and this was used as the criterion for a clinical cut-off. The modified Brinley plots show that two participants moved from the risk to the non-risk range, one participant had a non-risk score at both times and one participant's score improved but stayed in the risk range. The Reliable Change Index was also computed for the PDR child behaviour measure and this showed that none of the participant's scores were outside the RCI cut off $\left(R_{\text {crit }}=4.66\right)$, indicating that the level of change observed was not outside the range of measurement error. 
Table 11 Means, (Standard Deviations) at Pre-and Post-intervention, Effect sizes (Cohen's d) and Probability values for $t$ (2 tailed) comparing Pre and Post-intervention Means

\begin{tabular}{lcccc}
\hline Measure & $\begin{array}{l}\text { Pre-intervention } \\
\text { Mean }(S D)\end{array}$ & $\begin{array}{l}\text { Post- intervention } \\
\text { Mean }(S D)\end{array}$ & T test & $\begin{array}{l}\text { Effect size } \\
(\text { Cohen's } d)\end{array}$ \\
\hline Mind-mindedness & $0.22(.121)$ & $0.40(.129)$ & $-13.12^{* *}$ & 1.50 \\
Attributions & $3.71(1.02)$ & $4.63(0.17)$ & $-1.94^{\mathrm{t}}$ & 1.49 \\
PDR: Child & $6.8(2.23)$ & $3.8(3.29)$ & $3.62^{*}$ & 1.07 \\
Behaviour & & & & \\
PDR: Parent Stress & $15.4(8.72)$ & $10.3(10.09)$ & 1.33 & 0.54 \\
SDQ & $17(7.6)$ & $14.5(6.4)$ & 2.10 & 0.36 \\
DASS & $8.5(6.81)$ & $6(7.66)$ & 0.45 & 0.21 \\
PCOS Satisfaction & $42.5(4.12)$ & $41(1.82)$ & 0.97 & 0.51 \\
PCOS Efficacy & $34.25(6.75)$ & $32.75(5.9)$ & 1.73 & 0.24 \\
PESQ Dismissing & $31(3.6)$ & $31.6(2.08)$ & -0.36 & 0.21 \\
PESQ Coaching & $39.3(3.05)$ & $40.06(1.52)$ & -1.51 & 0.57 \\
PESQ Empathy & $16.6(2.08)$ & $17.6(2.3)$ & -1.00 & 0.23 \\
CPRS Closeness & $27.2(6.23)$ & $26.5(6.13)$ & 0.40 & 0.11 \\
CPRS Conflict & $23.7(12.12)$ & $23(5.31)$ & 0.29 & 0.08 \\
\hline
\end{tabular}

${ }^{\mathrm{t}} p<.01 ; * p<.05 ; * *<.01$ one tailed significance. PDR = Parent Daily Report Checklist.

The foster parent reported daily stress ratings also decreased from a mean of 15.4 to 10.3 but this was not statistically significant. Figure 8 shows that three out of four participants had decreased parent daily stress. As above, clinically significant change cannot be estimated due to a lack of population norms but, given that the cut off for risk of placement breakdown is six behaviours (Chamberlain et al., 2006) and the maximum score for parent stress for six behaviours is 12 , a cut off of 12 was used as our criterion. One participant had an improved score but remained in the risk range at post-intervention, one participant was in the non-risk range at both pre- and post-intervention, one participant moved from the risk range at pre-intervention to the non-risk range at post-intervention and one participant's stress deteriorated and was in the risk range at both pre- and postintervention. Regarding reliable change, only one participant scored outside the range of measurement error.

Strengths and Difficulties Questionnaire. The overall behavioural difficulties as measured by the Strengths and Difficulties questionnaire also dropped from 17 to 14.5, which did not reach statistical significance. Figure 8 shows that all participants reported a decrease in their foster child's overall emotional and behavioural problems. The criterion used for clinically significant change was the midpoint of the Australian population mean of 8.2 
(Mellor, 2005) and the mid-point between the current sample mean (12.6). Neither of the two participants who scored above the clinical cut-off moved into the non-clinical range at postintervention.

Depression, Anxiety, Stress Scale (DASS). The mean total score on the DASS decreased from 8.5 to 6 with two participants scoring zero at post-intervention but this result was not statistically significant. As can be seen on the modified Brinley Plot three participants had a decreased score and one score increased. All participants were below the clinical cut off at both pre- and post-intervention.

Other measures. There was virtually no change from pre- to post-intervention in three measures, namely the Child-Parent Relationship Scale scores, the Parent Sense of Competency Scale and the Parent Emotional Style Questionnaire. The resulting modified Brinley plots are shown in Figure 8 showing there was little variation between pre- and postintervention.

\section{Continuous measures}

The Parent Daily Report (PDR) measure yields two scores; the number of daily child problem behaviours (PDR: Child Behaviour) and the parent's rating of how much each behaviour upset them on a three point Likert scale (PDR: Parent Stress). Because these two scores were highly correlated $(r=.87, p<.001)$ only the PDR child behaviour scores were graphed. One parent only completed two data points at baseline, due to an administrative error. Below are the bi-weekly means of the PDR child behaviours scores, measured as part of the pre- and post-intervention and six month follow-up batteries for three days per week and then twice a week over the duration of the study (see Figure 9).

Three participants showed a downward slope over the baseline period. This compromises the integrity of the research design as a decreasing baseline indicates improved scores before the treatment phase began. However, none of the participants with a complete baseline had unacceptable variability (over 50\%) over the baseline phase. Participant 1 showed low daily behaviour problems over the second half of the baseline and during the treatment phase. Participant 2 was undergoing transition planning over the time of the intervention phase and during this process the placement broke down, due to factors unrelated to the group participant. Participant 2 showed high variability across the weeks (a range of 0 -10) and the score increased to 13 on the day of the placement disruption. Participant 3 showed an upward trend over the baseline period and problem behaviours were maintained at a high level, placing the placement at risk of disruption, until towards the end of the treatment 
phase when they began to decline. The foster parent reported this occurred after she used some of her emotion coaching skills with the young person and made a "breakthrough". This young person went on to make a successful transition to a permanent foster family.

Participant 4 had only 2 baseline measures and showed high variability (range $0-14$ ) and alternating weeks of a high number of problem behaviours $(10-14)$ and a low number of problem behaviours (0-1). The high scores were reported as due to a stressful incident occurring on an access visit to the young person's birth family.

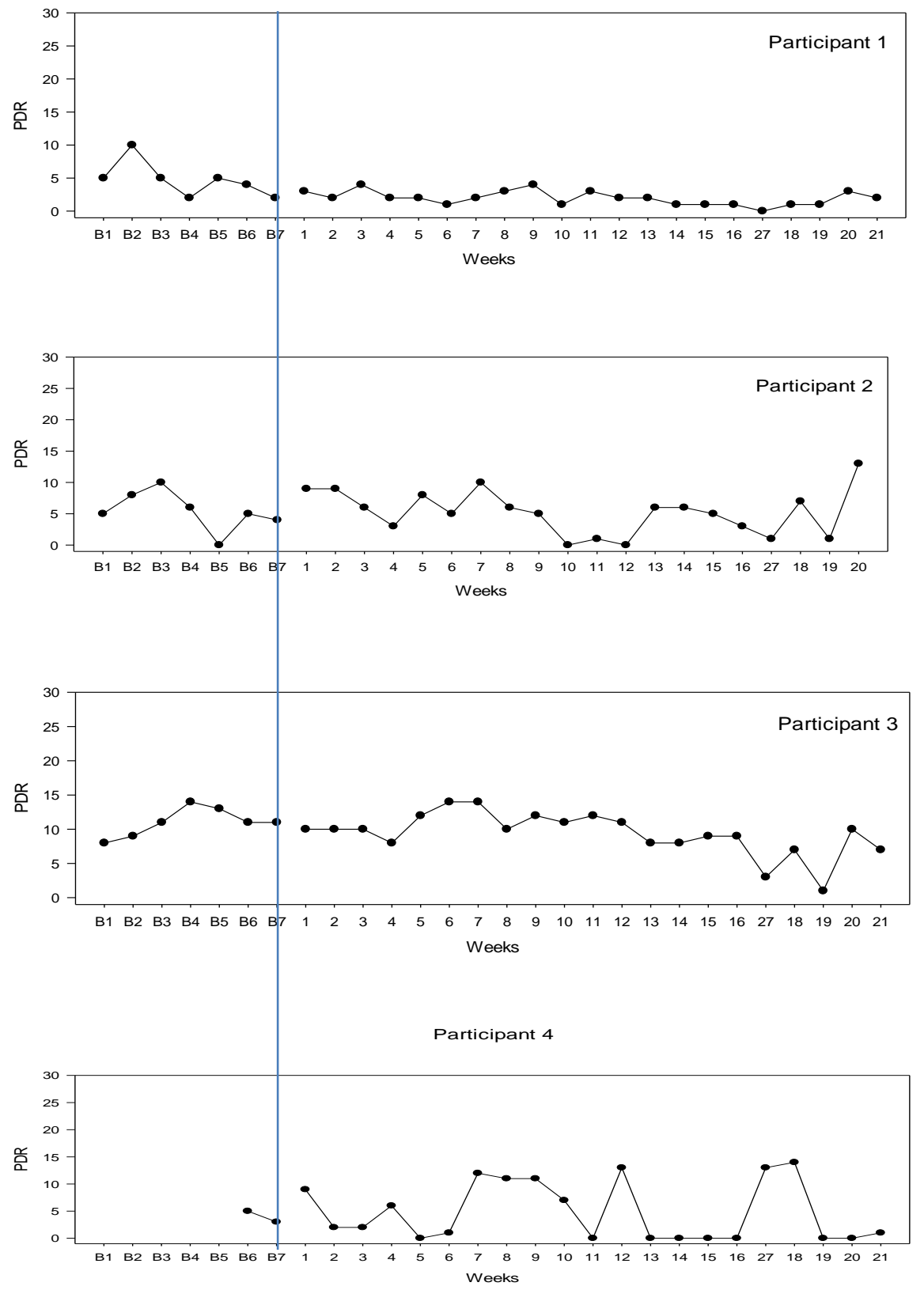

Figure 9. Level of Parent Daily Report: Child Behaviour for participants $1-4$. 
A treatment effect cannot be detected visually on the time-series graphs due to the downward sloping baselines and the variability of the scores over the treatment phase. However, Table 12 shows that $68.45 \%$ of the PDR scores in the treatment phase exceeded the median of the baseline, indicating a possible treatment effect. The "Percentage Exceeding the Mean (PEM) ranges from $33.33 \%$ to $100 \%(M=55.83)$ in the first half of the treatment phase and $60 \%$ to $100 \%(M=81.93)$ in the second half of the treatment phase, indicating more improvement in the second half of the treatment phase.

Table 12 Percentage of Treatment Scores Exceeding the Median of the Baseline for the First and Second Halves of the Treatment Phase and Overall

\begin{tabular}{llll}
\hline & PEM $1^{\text {st }} 1 / 2$ & PEM $2^{\text {nd }} 1 / 2$ & PEM Total \\
\hline Participant 1 & 33.33 & 60 & 46.67 \\
Participant 2 & 100 & 100 & 100 \\
Participant 3 & 50 & 90 & 70 \\
Participant 4 & 40 & 77.73 & 57.14 \\
\hline Mean & 55.83 & $81.93^{\mathrm{t}}$ & 68.45
\end{tabular}

${ }^{\mathrm{t}} p=.065$

\section{Discussion}

The first question addressed was whether the intervention changed the target measures of mind-mindedness and attributions. It was expected that the group intervention would increase foster parents' scores on mind-mindedness and attributions, as these were proposed to be impacted by the group content which aimed to increase parents' psychological awareness of the child. The first hypothesis was partially supported with a significant increase in parental mind-mindedness and a non-significant increase in attribution scores. The effect size for mind-mindedness was large and indicates that parents talked more about their young person's mental states and emotions in the post-intervention interview than they did in the pre-intervention interview. The increase in attribution score showed that foster parents were more willing to attribute the young person's behaviour to a range of causes, although this change did not reach statistical significance.

The second question was whether there were effects on the child's behaviour, the parent's stress, emotion style, depression, anxiety and stress, sense of competence and the parent child relationship. It was hypothesised that there would be associated positive changes 
changes in children's behaviour problems, foster parent well-being and the foster parent-child relationship. This hypothesis was also only partially supported. The only measure which showed a significant decrease was the PDR: Child Behaviour, with the mean score moving to the non-risk range (Chamberlain et al., 2006). The mean Strengths and Difficulties Questionnaire and the Depression, Anxiety and Stress Scale scores showed decreases but these were not statistically or clinically significant. Three other measures showed little or no change, namely the Child-Parent Relationship Scale, the Parent Sense of Competency Scale and the Parent Emotional Style Questionnaire.

The mean scores for parent satisfaction and efficacy for participants in this study before the intervention were considerably higher than for parents in the community (Johnston \& Mash, 1989; Ohan, Leung, \& Johnston, 2000) and were higher than the post-intervention scores of a group of parents at risk of maltreating their young children (Sanders et al., 2004). This suggests that the parents in the pilot group may have already felt satisfied and effective in their role, having raised several children of their own. As foster parents, it is also possible that the parents may have seen parenting difficulties as largely reflecting the child's problems rather than their own adequacy. In addition, this scale was developed for children and may not be suitable for adolescents. Similarly, the Parent Emotion Style Questionnaire was developed for and validated with pre-schoolers (Havighurst et al., 2010; Lagacé-Séguin \& Coplan, 2005) and may not be suitable for older children and adolescents. The means for the pilot group parents were similar to the means for 218 parents of pre-schoolers (Havighurst, Wilson, Harley, \& Prior, 2009) and it is also possible that the parents simply did not improve on this aspect of parenting.

It is not possible to draw firm conclusions about why the Child-Parent Relationship Scale did not show any change as the studies using it have either not reported means and standard deviations or it is unclear what version of the CPRS was used (NICHD Early Child Care Research Network, 2004; Searle, Miller-Lewis, Sawyer, \& Baghurst, 2013).

In light of the results of the pilot study, these three questionnaires were dropped from the subsequent study as they either showed no change or were not developed for the age group of the foster children. Several questionnaires were added. The Outcome Rating Scale was developed as a global measure of therapy outcome (Miller, Duncan, Brown, Sparks, \& Claud, 2003). In addition two questionnaires which have been shown to be useful in foster care treatment outcome research were added. These were the Quality of Attachment Relationship Questionnaire, which was developed from attachment theory with good face validity, and the Carer-defined Problems Scale, which appears to be sensitive to behaviour 
change in foster children (Briskman et al., 2012). Finally, a measure of caregiver strain was added, the Caregiver Strain Questionnaire (Brannan, Heflinger, \& Bickman, 1997) and an extra coding schedule was applied to the behavioural incident the foster parent described in the attributions measure. These measures are outlined in Study 2(b). 


\section{Study 2 (b) Main Group}

\section{Participants}

The participants in study 2 (b) took part in one of four groups. Groups 1 and 2 were conducted at a residential facility where children live with either one or two foster parents and some care assistants, mostly as large sibling groups (see Table 13). All caregivers had multiple children in their care (between five and nine children) and were requested to choose the child for the study whose behaviour they were most concerned about. The children were five girls and three boys aged between two and 13 years and were of Māori, Tongan, and Fiji Indian descent. They had lived with their caregivers between three months and three and a half years. The caregivers were of Māori, Māori/Cook Island, Samoan, and NZ European descent. Cultural issues, such as appropriate introductions to the groups and how to help a child identify more positively with their own culture, were reviewed by the author in cultural supervision with a Māori clinical psychologist.

The groups consisted of four five hourly sessions, which were planned to be spaced a month apart. Eleven foster parents started the group but three were not included in the completed research because one attended only one session, one targeted adolescent absconded on the first day of the group and one participant died suddenly between the first and second group sessions. This tragic event resulted in a delay of two months between the first and second training days and is likely to have also had a detrimental impact on the other caregivers and the children in their care. The subsequent three training days were held fortnightly, due to this earlier delay.

Table 13 Demographic Information of Participants in Group 1and 2

\begin{tabular}{|c|c|c|c|c|c|c|}
\hline \multicolumn{3}{|c|}{ Foster parent } & \multicolumn{4}{|c|}{ Foster child } \\
\hline Ethnicity & Age & Gender & Ethnicity & Gender & Age & Months placed \\
\hline Māori & 47 & Female & Māori & Female & 11 years & 15 \\
\hline Māori & 61 & Female & Māori & Female & 5 years & 3 \\
\hline Māori/ Cook Is. & 43 & Female & Tongan & Male & 6 years & 11 \\
\hline Samoan & 51 & Female & Tongan & Male & 2 years & 11 \\
\hline NZ European & 54 & Male & Fijian & Male & 12 years & 24 \\
\hline Samoan & 41 & Female & Māori & Female & 11 years & 2 \\
\hline Māori & 53 & Female & Tongan & Female & 13 years & 3 \\
\hline NZ European & 52 & Male & Māori/Pacific & Female & 5 years & 42 \\
\hline
\end{tabular}


Group 3 was conducted at the same agency as the pilot group described above. This group contained young people who had high levels of externalising behaviour including running away, burglary and assault, with Youth Court involvement. One had been diagnosed with Asperger's syndrome. The young people were aged between 13 and 15 years (see Table $14)$.

Table 14 Demographic Information of Participants in Group 3 (Same Agency as Pilot Group)

\begin{tabular}{|c|c|c|c|c|c|c|}
\hline \multicolumn{3}{|c|}{ Foster parent } & \multicolumn{4}{|c|}{ Foster child } \\
\hline Ethnicity & Age & Gender & Ethnicity & Age & Gender & Months placed \\
\hline NZ Eur. & 51 & Female & NZ Eur. & 13 & Female & 1.5 \\
\hline Māori & 48 & Female & Māori & 16 & Male & 24 \\
\hline Māori & 50 & Female & Māori & 15 & Female & 1 \\
\hline NZ Eur. & 60 & Male & NZ Eur. & 15 & Male & 4 \\
\hline
\end{tabular}

Group 4 was conducted at a Child and Adolescent Mental Health Service and was cofacilitated by a staff member from the service. All of participants in this group were family/whānau caregivers of the children (see Table 15). Due to the social circumstances of this group, including isolation and poor financial circumstances, three meetings were conducted in the follow-up period to provide further practice at applying the group principles. No new material was offered. One person dropped out of this group due to moving to another district.

In total, 15 participants completed the training programme and the post-test measures and nine completed the six month follow-up measures, due to the transitioning of the child or adolescent from the foster parent's care or inability to contact the foster parent.

Table 15 Demographic Information of Participants in Group 4

\begin{tabular}{|c|c|c|c|c|c|c|}
\hline \multicolumn{3}{|c|}{ Foster parent } & \multicolumn{4}{|c|}{ Foster child } \\
\hline Ethnicity & Age & Gender & Ethnicity & Age & Gender & Months \\
\hline NZ Eur. & 54 & Female & NZ Eur./Māori & Male & $10 y$ & 8 \\
\hline NZ Eur. & 69 & Female & NZ Eur. & Female & $8 \mathrm{y}$ & 43 \\
\hline NZ Eur. & 59 & Female & NZ Eur./Samoan & Female & 16 years & 120 \\
\hline
\end{tabular}

\section{Method}

The research design and method has been described above in study 2(a). However, three measures were dropped after the pilot group data was analysed. These were the Parent Emotion Style Questionnaire, the Parent Sense of Competency Scale and the Child-Parent 
Relationship Scale. Thus the measures retained for study 2(b) were the demographic and mind-mindedness interviews, the Parent Daily Report (child behaviour and parent stress), the Depression, Anxiety and Stress Scale and the Strengths and Difficulties Questionnaire. Four additional questionnaires were added as follows.

Quality of Attachment Relationship Questionnaire. This questionnaire has been designed for use with foster children to evaluate the outcomes of group therapy with foster parents. The questionnaire includes questions about whether the foster child approaches the caregiver when not feeling well, talks to the caregiver, shares affection, and whether the foster parent is able to comfort the child and help them manage feelings. The questionnaire does not have validity information available (Briskman, J. personal communication). Chronbach's alphas for the current study were $\alpha=.90$ at pre-test, $\alpha=.88$ at post-test and $\alpha=$ .93 at six month follow-up.

Caregiver Strain Questionaire. (CGSQ; Brannan, Heflinger, \& Bickman, 1997). This questionnaire was designed to measure the experiences of parents with children who have emotional and behavioural difficulties. It has three subscales, Objective strain which includes difficulties managing the child; Internalised Subjective Strain such as worrying about the child or the future; and Internalised Subjective Strain which includes feelings of anger and resentment towards the child. The scale correlated as expected with measures of family functioning and the caregiver's psychological well-being and the internal consistency was high $(\alpha=.93)$. Chronbach's alphas for the current study were $\alpha=.96$ at pre-test, $\alpha .=97$ at post-test and $\alpha=.96$ at six month follow-up.

Outcome Rating Scale. (ORS; Miller, Duncan, Brown, Sparks, \& Claud, 2003). This measure consists of a series of four $10 \mathrm{~cm}$ visual analogue scales on which the individual or parent places a mark to reflect the child's functioning individually (personal well-being), interpersonally (family, close relationships), socially (work, school, friendships) and overall functioning (general sense of well-being). The ORS has excellent internal reliability in a community sample of adults with internal consistency alphas of $.87-.96$ but test re-test reliability is somewhat lower (.49 - .66), which could be due to its sensitivity to change (Miller et al., 2003). Moderate correlations of .59 were obtained comparing the ORS to an established outcome scale, the Outcome Questionnaire 45.2, and the ORS was also sensitive to change in a clinical sample undergoing therapy, compared to a community sample (Miller et al., 2003).

Carer-defined Problems Scale. (Scott, Spender, Doolan, Jacobs, \& Aspland, 2001). This measure asks parents to write down their foster child's three biggest problems and then 
mark a $10 \mathrm{~cm}$ visual analogue scale to indicate the severity of the problem. Because this scale was part of the parent's workbook there was no follow-up data for this measure and although little validity data is available, it has been used in several studies (e.g., Warman et al., 2006)

Behavioural incident interview. The attributions measure required parents to talk about "a time the child showed difficult behaviour and or got into trouble". This interview was transcribed and coded using the Articulated Thoughts in Simulated Situations paradigm (Zanov \& Davison, 2010) outlined in Chapter 8, with the difference that the topic for the current study was not a simulated situation but was an incident reported by the foster parent. Empathic statements, references to past or current causes of the behavioural incident, statements referring to the family as a secure base and putting thoughts about the child into words were coded. The coding system developed is shown in Appendix D. Double coding of $30 \%$ of the transcripts produced a kappa of $\kappa=.79$.

\section{Results}

\section{Treatment evaluation}

Three participants did not complete the groups (16\%) and one participant was deceased. Attendance rates were high, with five participants missing one session, which was made up at a later date. Consumer satisfaction with the group was also high. Participants were asked to rate the training programme on a Likert scale from 1 (not useful) to 5 (extremely useful). Eleven participants $(73 \%)$ gave the maximum rating and the mean was $4.79(S D=0.43)$. The participants were then asked if the programme changed the way they thought about their foster child. Again ratings were high with nine participants giving the highest ranking, with a mean of $4.36(S D=1.15)$. Participants were asked whether the programme changed the way they interacted or behaved with their foster child and eight gave the highest rating of "a great deal", with a mean of $4.36(S D=.93)$. Participants were asked how much the foster child responded to this change and fewer participants (five) gave the maximum rating of "a great deal" $(M=4.00, S D=.96)$. Participants were asked if they would recommend the group to a friend and 10 gave the maximum rating with a mean of $4.64(S D=.63)$.

Qualitative comments made by the participants include "I step back and observe the behaviour of the young person more. Stopped to think about what they wanted to say and what was important" and "Most certainly it has been very helpful...the brainstorms and sharing times, being more positive, realising that I have to trust in her again". Other comments included "It is a great understanding of the young person and myself" and "It 
helped me immensely to have empathy for her - where she is coming from and to talk to her more. Another participant said that "It made me understand where the child came from and how much "baggage" the child had to cope with" and "I became more tolerant towards him and his behaviour and concentrated on all the positive things that have changed instead of the negative".

\section{Repeated measures}

The mean pre- and post- intervention scores, paired samples t-tests (one tailed) and effect sizes are shown in Tables 16 and 17.

Table 16 Mean, Standard Deviations, $t$-tests and Effect sizes for Mind-mindedness and the Behavioural Incident Interview at Pre- and Post-interventionfor all groups (1-4)

\begin{tabular}{lllll}
\hline Measure & $\begin{array}{l}\text { Mean }(S D) \text { Pre- } \\
\text { intervention }\end{array}$ & $\begin{array}{l}\text { Mean }(S D) \text { Post- } \\
\text { intervention }\end{array}$ & T test & Effect size \\
\hline Mind-mindedness & $0.36(0.25)$ & $0.34(0.13)$ & 0.17 & 0.01 \\
Positive MM & $0.38(0.28)$ & $0.47(0.32)$ & -0.64 & 0.30 \\
Negative MM & $0.46(0.33)$ & $0.33(0.29)$ & 0.90 & 0.31 \\
Reflection & $8.3(4.17)$ & $9.7(5.15)$ & -0.89 & 0.30
\end{tabular}

${ }^{\mathrm{t}} p<.01 ; * p<.05 ; * * p<.01 \mathrm{MM}=$ mind-mindedness

Table 17 Mean, Standard Deviations, t-tests and Effect sizes for Mind-mindedness and the Behavioural Incident Interview for the Follow-up Group at Pre-intervention and 6 Month Follow-up

\begin{tabular}{lcccc}
\hline Measure & $\begin{array}{l}\text { Mean }(S D) \text { Pre-intervention } \\
\text { follow-up group }\end{array}$ & $\begin{array}{l}\text { Mean }(S D) \text { Pre- } \\
\text { intervention } \\
\text { follow-up group }\end{array}$ & $t$ test & Effect size \\
\hline Mind-mindedness & $0.33(0.32)$ & $0.35(0.17)$ & -0.35 & 0.08 \\
Positive MM & $0.39(0.35)$ & $0.65(0.36)$ & $-2.73^{*}$ & 0.74 \\
Negative MM & $0.40(0.40)$ & $0.18(0.35)$ & 1.01 & 0.38 \\
Reflection & $6.62(4.17)$ & $9.75(2.96)$ & 1.66 & 0.88 \\
\hline
\end{tabular}

${ }^{\mathrm{t}} p<.01 ; * p<.05 ; * * p<.01 \mathrm{MM}=$ mind-mindedness

Mind-mindedness. There were no significant differences between the proportion of mind-related comments or the negative and positive mind-related comments foster parents made from pre- to post-intervention (see Table 17). There was a significant increase in positive mind-related comments from pre-intervention to six month follow-up with a medium effect size (see Table 18). Modified Brinley plots (see Figure 10) reveal a spread of data points both above and below the diagonal, showing no treatment effect for mind-related comments or the valence of the comments (positive or negative). Using the mid-point of two 
community mean scores as an indicator of population functioning, nine participants were at or below this mean at pre-test and remained so at post-test, again indicating a lack of treatment effect. While only positive mind-related comments showed a significant change at six month follow-up, a visual inspection of the modified Brinley plots show that a majority had increased numbers of mind-related and positive mid-related comments and decreased negative mind-related comments at follow-up, compared to pre-intervention.
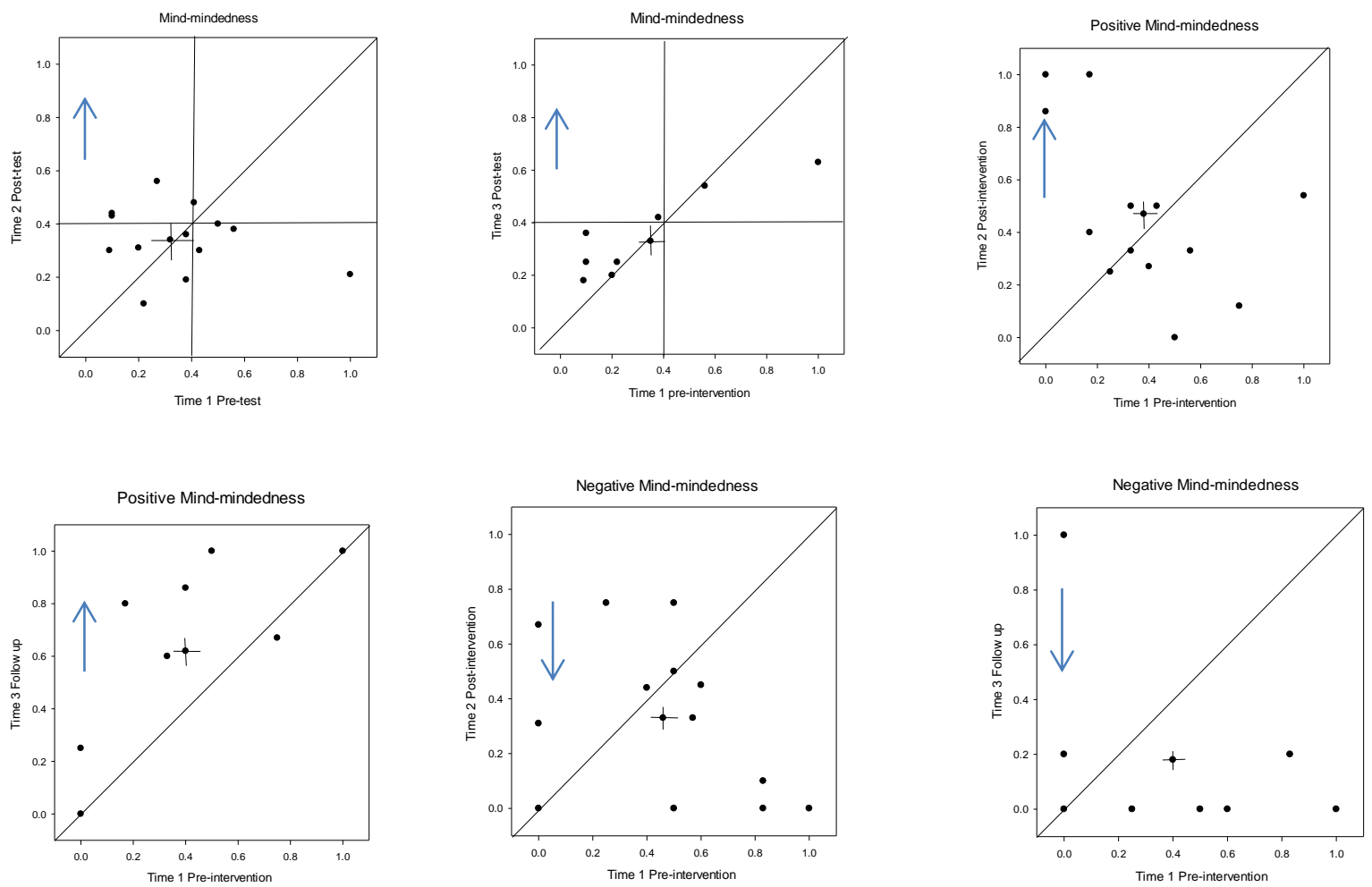

Figure 10. Modified Brinley plots showing pre-intervention scores for mind-mindedness, positive mind-mindedness and negative mind-mindedness on the $\mathrm{X}$ axis and post-intervention or follow-up scores on the Y axis.

Note: Each point represents one participant. The mean is indicated by a cross. Arrows indicate direction of improvement.

Behavioural incident interview. Parents' interviews about a behavioural incident were coded using the Articulated Thoughts in Simulated Situations Paradigm. Two participants did not complete the interview at post-intervention and six month follow-up because their child had not "shown naughty behaviour or got into trouble". Scores were calculated for the total number of empathic or reflective comments about the causes of the child's behaviour and the proportion of such comments compared to overall word count (to control for verbosity). From pre- to post-intervention there was a statistically non-significant 
increase in the total empathic and reflective comments from a total of $8.3(S D=4.17)$ to a total of $9.7(S D=5.15)$. For those who completed follow-up, there was also a statistically non-significant increase between 6.6 and 9.75 from pre-intervention to follow-up. At an individual level examining modified Brinley plots (Figure 11), nine participants had increased empathic and reflective comments, one stayed the same and three had lower scores. At six month follow-up, five participants had increased scores, two participants had decreased scores and one had stayed the same. These plots suggest a possible treatment effect.
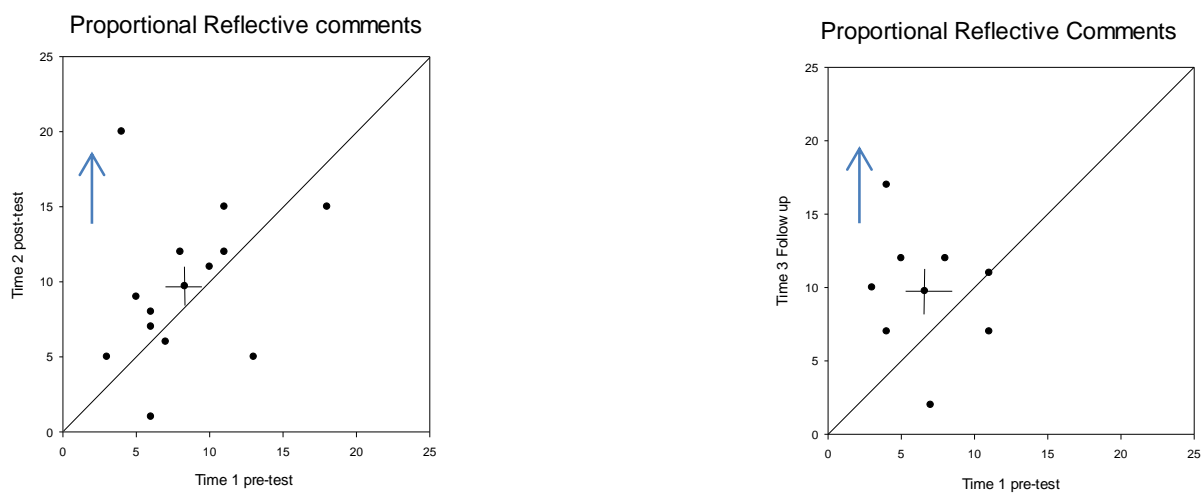

Figure 11. Modified Brinley plots showing time 1 pre-intervention scores for the Behavioural Incident Interview on the $\mathrm{X}$ axis and post-intervention and follow-up scores on the $\mathrm{Y}$ axis.

Note: Each point represents one participant. The mean is indicated by a cross.

Attributions. The average rating of attributions participants endorsed were not available for all participants due to some being unable to identify an episode of difficult behaviour. However, differences between the average ratings of attributions pre- and postintervention were statistically significant at the .10 (one tailed) level and there were no significant differences at six month follow-up (see Table18 and 19). 

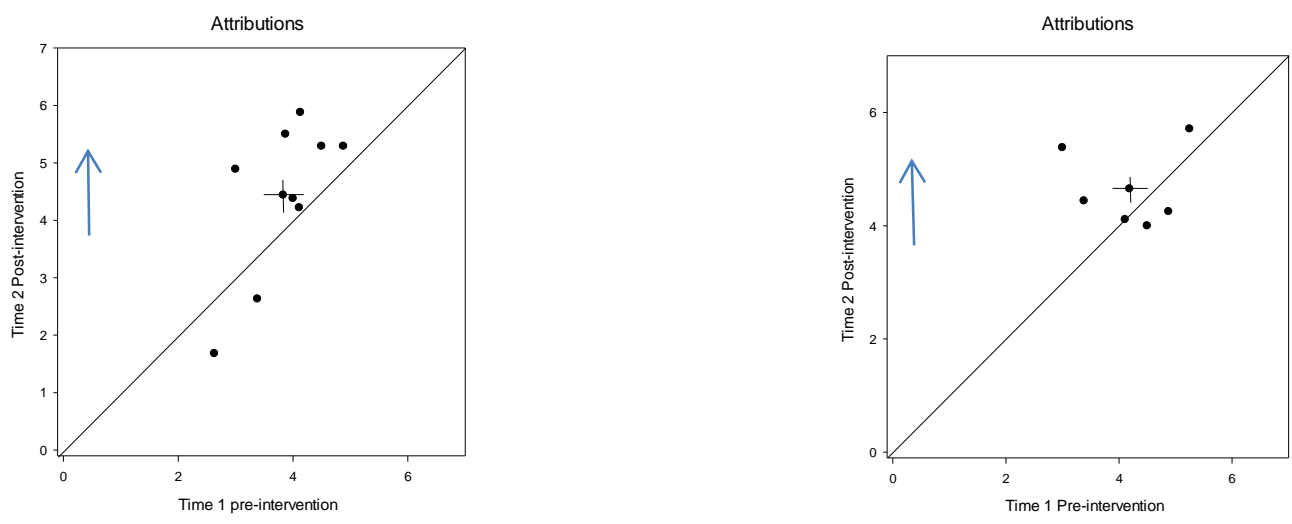

Figure 12. Modified Brinley plots showing pre-intervention scores for Attributions on the $\mathrm{X}$ axis and post-intervention and follow-up scores on the $\mathrm{Y}$ axis.

Strengths and Difficulties Questionnaire. Chronbach's alphas for each subscale of the SDQ ranged from poor (peer subscale at pre-intervention $\alpha=0.30$ ) to high (emotion subscale at post-test $\alpha=0.85$ ). Alphas may be expected to be low for subscales with low numbers of items (Cortina, 1993) but the alpha for the total score was $\alpha=.69$ and only the total score was used in calculations. There was a statistically significant decrease in reported child behaviour problems from pre-to post- intervention of $0.62 S D$ and a medium effect size (Cohen's $d=0.54$ ). Modified Brinley plots (see Figure 6) show that 11 participants (73\%) had decreased scores at post-intervention and five participants $(56 \%)$ at six month follow-up.

Table 18 Means, Standard Deviations and t-tests from Pre-to Post-intervention $(N=15)$

\begin{tabular}{lccccl}
\hline & Mean $(S D)$ T1 & Mean $(S D)$ T2 & Difference & t-tests & Effect size \\
\hline PDR-B & $11.27(6.34)$ & $7.54(7.92)$ & -3.73 & $3.97^{* *}$ & 0.52 \\
PDR-S & $15.21(12.18)$ & $5.60(7.89)$ & -9.61 & $4.07^{* *}$ & 0.96 \\
QUARQ & $38.79(9.98)$ & $47.93(11.08)$ & +9.14 & $-3.35^{* *}$ & 0.87 \\
CGSQ & $54.27(23.18)$ & $46.47(26.16)$ & -5.67 & 1.12 & 0.22 \\
SDQ & $19.13(5.46)$ & $15.73(7.05)$ & -3.40 & $2.16^{*}$ & 0.54 \\
DASS & $32.17(25.00)$ & $31.29(20.94)$ & -0.88 & -0.18 & 0.04 \\
ORS & $18.45(10.56)$ & $25.57(11.90)$ & +7.12 & $-2.04^{*}$ & 0.63 \\
Attributions & $3.83(.71)$ & $4.44(1.36)$ & +0.61 & -1.80 & 0.58 \\
CDP & $70.82(23.28)$ & $38.17(23.84)$ & -32.65 & $4.22^{*}$ & 1.39 \\
& & & & & \\
\hline
\end{tabular}

${ }^{*} p<.05, * * p<.01$, one tailed significance. PDR-B = Parent Daily Report Checklist(Child behaviour); PDR-S = Parent Daily Report Checklist (parent stress); QUARQ = Quality of Attachment Relationship Questionnaire; CGSQ = Caregiver Strain Questionnaire; SDQ = Strengths and Difficulties Questionnaire; DASS = Depression, Anxiety and Stress Scale $(N=12)$; ORS = Outcome Rating Scale; Attributions $(N=13)$; CDP $=$ Carer defined problems. 
Before the intervention, two participants scored below and four scored on the clinical cut-off, using the UK-normed cut-off score of 16. At post-intervention eight participants scored below the clinical cut-off and at six month follow-up three scored below and one scored on the clinical cut-off.

Table 19 Means, Standard Deviations and t-tests for Pre-intervention (Follow-up Group Only) to Follow-up $(N=9)$

\begin{tabular}{llllll}
\hline Measure & $\begin{array}{l}\text { Mean (SD) pre- test } \\
\text { follow-up group only }\end{array}$ & Mean (SD) Follow-up & Difference & t-test & Effect size \\
\hline PDR - B & $10.00(6.11)$ & $11.37(6.36)$ & $+1.37^{\mathrm{a}}$ & $-0.49^{\mathrm{a}}$ & $0.22^{\mathrm{a}}$ \\
PDR-S & $13.56(12.44)$ & $15.69(11.73)$ & $+2.13^{\mathrm{a}}$ & $-0.25^{\mathrm{a}}$ & $0.18^{\mathrm{a}}$ \\
QUARQ & $42.88(11.08)$ & $46.88(11.76)$ & +4.00 & -0.88 & 0.35 \\
SDQ & $20.00(4.66)$ & $16.78(7.97)$ & -3.22 & 1.15 & 0.51 \\
DASS & $32.00(20.70)$ & $45.33(35.04)$ & $+13.33^{\mathrm{a}}$ & $-1.51^{\mathrm{a}}$ & $0.46^{\mathrm{a}}$ \\
CGSQ & $59(26.09)$ & $50.67(22.83)$ & -8.33 & 0.90 & 0.34 \\
ORS & $17.7(11.04)$ & $28.03(7.78)$ & +10.33 & $-2.51^{*}$ & 1.10 \\
Attributions & $4.19(0.87)$ & $4.65(0.72)$ & 0.46 & -1.00 & 0.58 \\
\hline
\end{tabular}

${ }^{*} p<.05,{ }^{* *} p<.01$, one tailed significance. ${ }^{a}$ denotes deterioration. PDR-B = Parent Daily Report Checklist(Child behaviour); PDR-S = Parent Daily Report Checklist (parent stress); QUARQ = Quality of Attachment Relationship Questionnaire; CGSQ = Caregiver Strain Questionnaire; SDQ = Strengths and Difficulties Questionnaire; DASS = Depression, Anxiety and Stress Scale; ORS = Outcome Rating Scale; Attributions ( $=8)$.

The reliable change index for the SDQ was calculated using the test-retest reliability value of .81 found by Achenbach et al. (2008) which yielded an RCI of $R_{\text {crit }}=6.95$. This is shown by the dotted parallel lines on the Modifed Brinley Plot. These indicate that only four participants $(27 \%)$ had change scores outside the range of measurement error, indicating reliable improvement, and one participant (7\%) showed reliable deterioration. At six month follow-up, four out of nine participants (44\%) showed statistically reliable improvement and one showed deterioration (11\%). This finding suggests that some of the apparent change in the participants scores may be a result of the reliability of the measure.

The criterion used for clinically significant change was the midpoint of the Australian population mean of 8.2 (Mellor, 2005) and the mid-point between the current sample mean (13.7). Clinically significant change was shown by five participants (33.33\%) at postintervention and three participants (33.33\%) at six month follow-up. The mean for pre- and post-intervention scores is close to the margin of the measurement error line and below the clinical cut-off. 

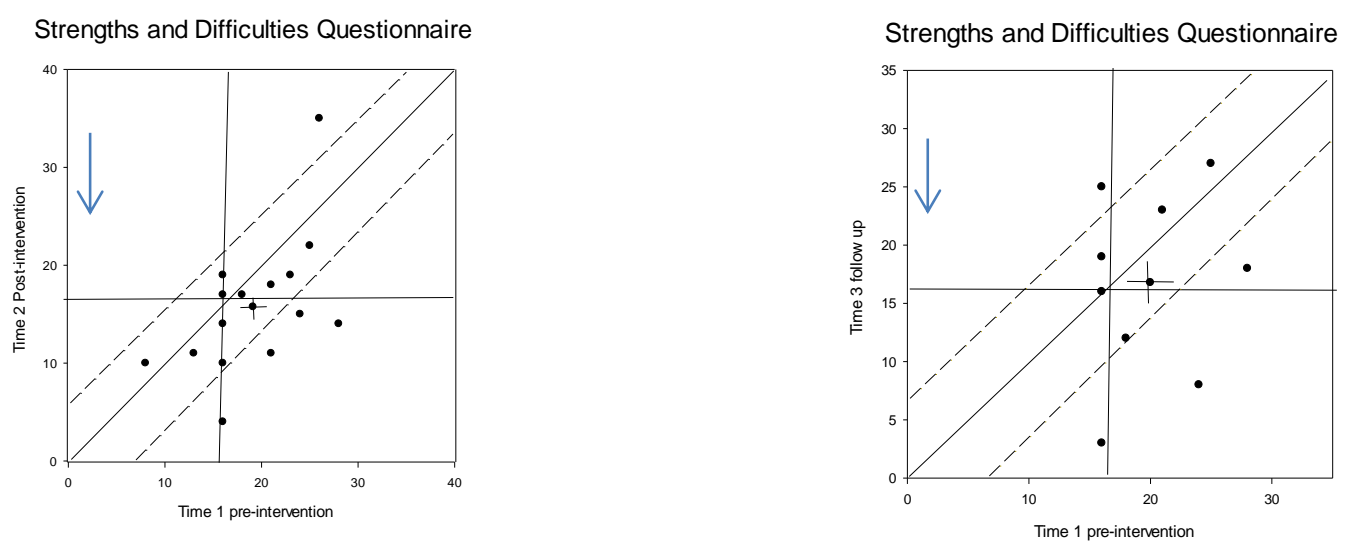

Figure 13. Modified Brinley plots showing pre-intervention scores for the Strengths and Difficulties Questionnaire on the $\mathrm{X}$ axis and post-intervention and follow-up scores on the $\mathrm{Y}$ axis.

The parellel lines indicate the area of measurement error.

Parent Daily report: Child behaviour. There was a statistically significant decrease in reported child behaviour problems from pre-group to post-group of $0.59 \mathrm{SD}$ and a medium effect size (Cohen's $d=0.52$ ). Using a cut-off for risk of placement disruption of six daily behaviours, at post-intervention nine participants $(67 \%)$ moved from the risk to non- risk range and three improved but remained in the risk range. At six month follow-up, six children remained in the risk of placement disruption range, one moved into this range and one was in the non-risk of placement disruption range. Only two children had maintained improved scores from pre-intervention and six had deteriorated from pre-intervention. These results suggest that the intervention had a treatment effect on daily behaviour problems for all but two of the children but this was lost by the six month follow-up when the mean child problem behaviour had increased by 0.2 standard deviations.

The Reliable Change Index was calculated using data from Hurlbert, Chamberlain, DeGamo and Zhang $(2010$; reported test re-test reliability $=.85)$. From pre-intervention to post-intervention $54 \%$ of the participants showed reliable improvement, $7 \%$ showed reliable deterioration and $46 \%$ showed no change. However, from pre-intervention to follow-up, only $12.5 \%$ showed reliable improvement, $12.5 \%$ reliable deterioration and $75 \%$ did not show reliable change. 

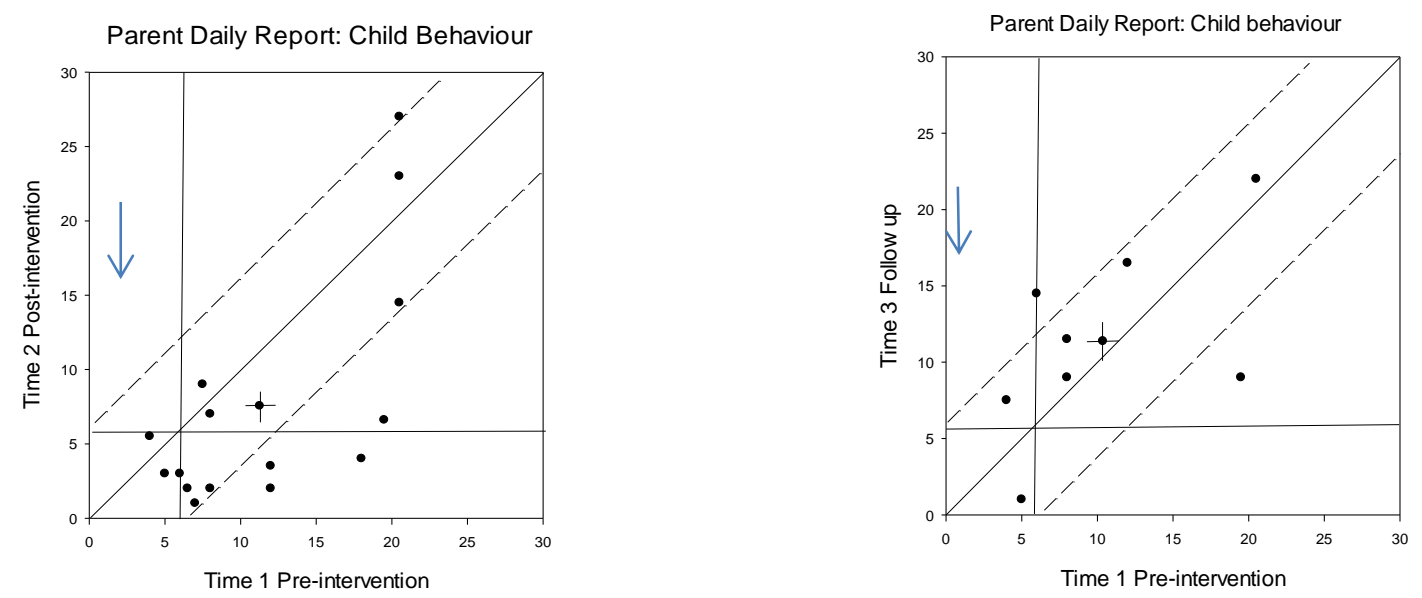

Figure 14. Modified Brinley plots showing pre-intervention scores for the Parent Daily Report: Child behaviour on the $\mathrm{X}$ axis and post-intervention and follow-up scores on the $\mathrm{Y}$ axis.

Parent daily report: Parent stress. There was a statistically significant decrease in reported parent daily stress of $0.79 S D$ and a large effect size of 0.94 (see Table 18).

Modified Brinley plots show that all parents except one reported lower daily stress levels from pre-intervention to post-intervention but at follow-up, five parents reported higher daily stress levels than at baseline and three reported lower levels (see Figure 15). Using 12 as the clinical cut off for parent stress, $60 \%$ scored under this cut-off at pre-intervention, $80 \%$ at post-intervention but only $44 \%$ did so at follow-up. These results suggest that gains at postintervention were lost by six month follow-up when the mean parent daily stress levels had increased by $0.17 S D$.

As above, the Reliable Change Index was calculated using test-retest reliability data from Hurlbert et al., (2010). At post-intervention only two participants (13\%) had reliably improved and $87 \%$ showed no reliable change. At six month follow-up, only one participant (11\%) showed reliable improvement, two (22\%) showed reliable deterioration and six (67\%) showed no reliable change. 

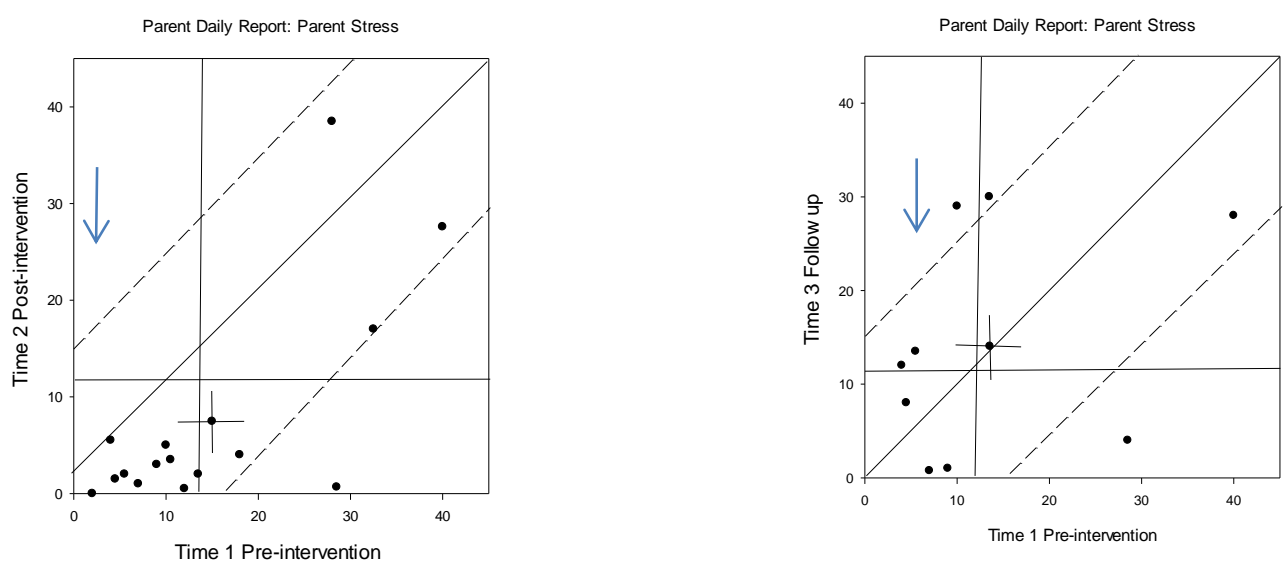

Figure 15. Modified Brinley plots showing pre-intervention scores for the Parent Daily Report: Parent Stress on the $\mathrm{X}$ axis and post-intervention and follow-up scores on the $\mathrm{Y}$ axis.

Outcome Rating Scale. On the Outcome Rating Scale, parents rate their child's functioning on a $10 \mathrm{~cm}$ Likert scale for interpersonal, family, school and overall domains. There was a significant increase in the functioning of participant's foster children from preintervention to post-intervention and also to six month follow-up (see Table 18 and 19). The pre-intervention mean increased by 0.68 standard deviations at post-intervention.

In a normative study (Duncan et al., 2006) the Outcome Rating Scale mean for the nonclinical group was 33.4 and the mean for the clinical group was 21.1. Using Jacobson and Truax's option (c), the cut-off indicating a participant is "recovered" is halfway between the mean for the clinical and non-clinical populations i.e., a scale of 27.25. Eleven participants $(77 \%)$ scored below this point at pre-intervention and this reduced to six participants $(40 \%)$ at post intervention. For the follow-up group, seven participants (78\%) scored below this point and this reduced to two participants (22\%) at six month follow-up (see Table 19). However, using the Reliable Change Index, only $27 \%$ of the participants scored in the recovered category, no participants deteriorated and $73 \%$ showed no reliable change. 

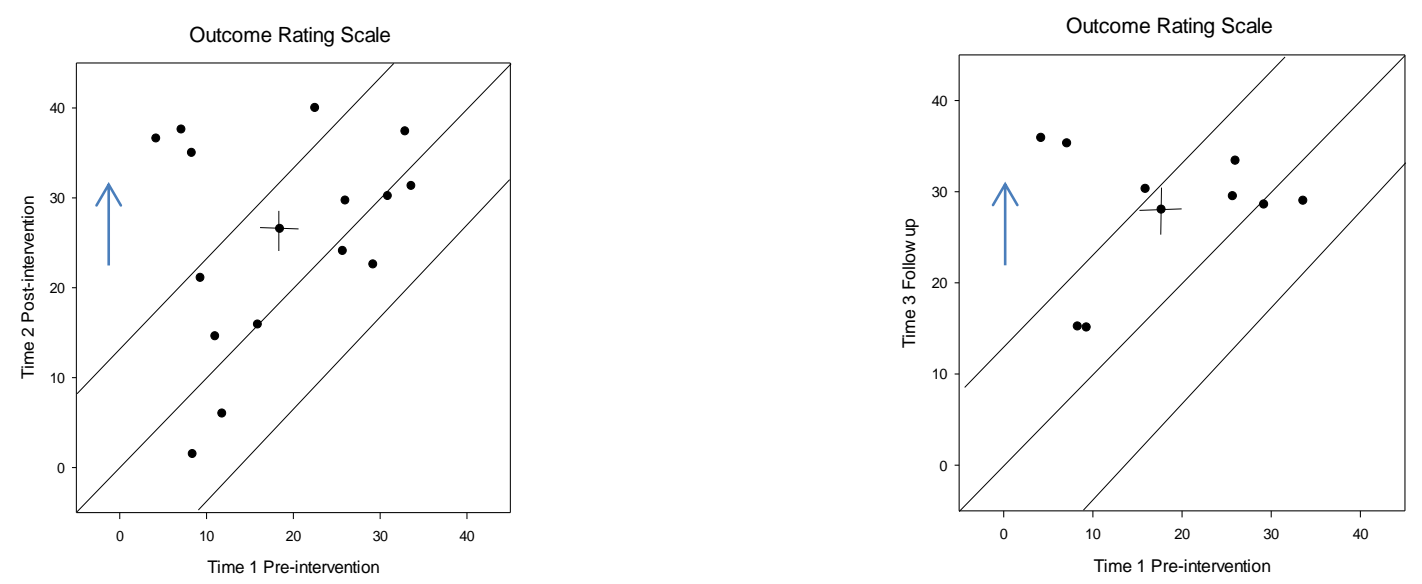

Figure 16. Modified Brinley plots showing pre-intervention scores for the Outcome Rating Scale on the $\mathrm{X}$ axis and post-intervention and follow-up scores on the $\mathrm{Y}$ axis.

Table 20 Number of Participants Showing Clinically Significant Change on the Outcome Rating Scale at Pre-intervention, Post-intervention and Follow-up

\begin{tabular}{lllcr}
\hline & Pre-intervention & Post-intervention & $\begin{array}{l}\text { Follow-up group pre- } \\
\text { intervention }(N=9)\end{array}$ & Follow-up $(N=9)$ \\
\hline Under 27.5 & $11(77.33 \%)$ & $6(40 \%)$ & $7(77.78 \%)$ & $2(22.22 \%)$ \\
Over 27.5 & $4(26.67)$ & $9(60 \%)$ & $2(22.22 \%)$ & $7(77.78 \%)$ \\
\hline
\end{tabular}

Carer defined problems. Foster parents were asked to rate the child's three biggest problems at the first group and at the final group they were reminded of their answers and asked to rate the child on this problem again. As this measure was part of the workbook it was not included at follow-up. The foster parent ratings of the severity of the child's problems fell from $71 \%$ to $38 \%$, which was a statistically significant difference.

Caregiver Strain Questionnaire. The mean Caregiver Strain Questionnaire score decreased by 0.42 standard deviations at post-intervention but this difference was not statistically significant. Modified Brinley plots show that pre- to post-intervention CGSQ scores decreased for four parents, stayed the same for four parents and increased for five parents (see Figure 10). At six month follow-up, scores had decreased from baseline for five parents and increased for four parents. These results suggest that the intervention did not have a treatment effect on caregiver strain and may have been deleterious for some parents. 

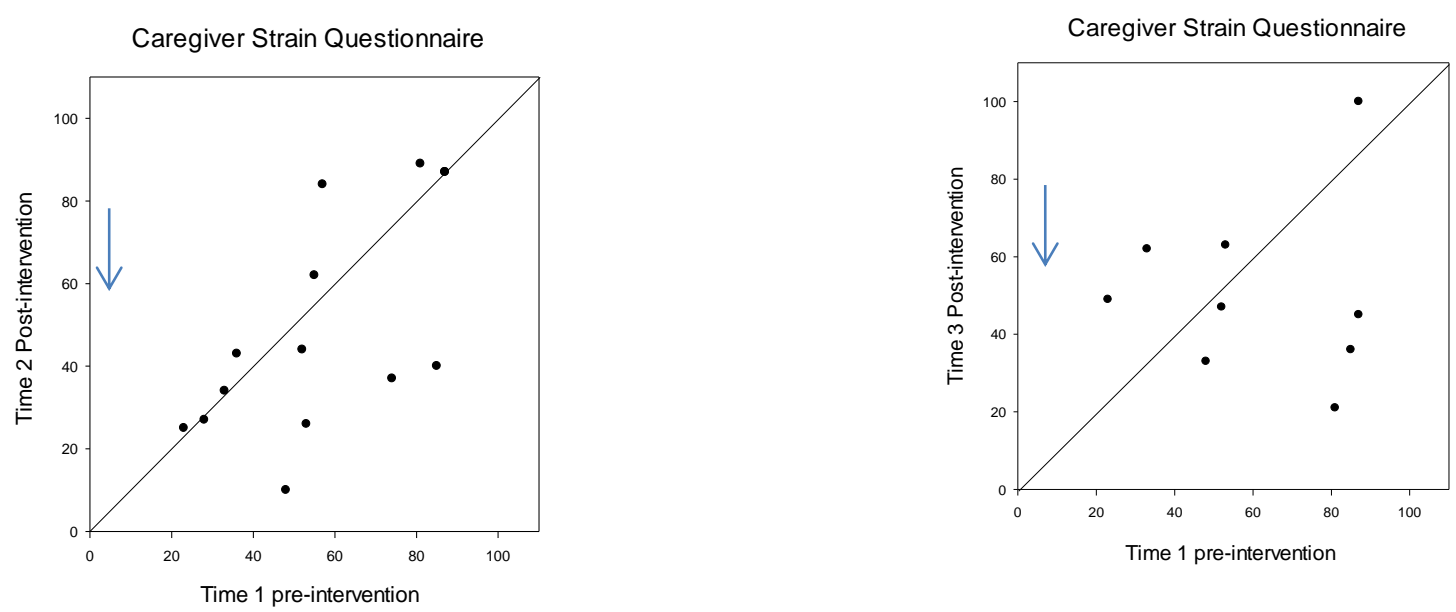

Figure 17. Modified Brinley plots showing time 1 pre-intervention scores for the Caregiver Strain Questionnaire on the $\mathrm{X}$ axis and time 2 and time 3 scores on the $\mathrm{Y}$ axis.

Quality of Attachment Relationship Questionnaire. The mean Quality of Attachment Relationship Questionnaire scores increased statistically significantly $(S D=$ 0.92 ) between pre- and post-intervention and the effect size was large (0.87). Modified Brinley plots showed that from pre- to post-intervention 12 participants had increased scores and two had decreased scores (see Figure 7.11). At six month follow-up seven participants had improved scores and one had a decreased score (27.25).
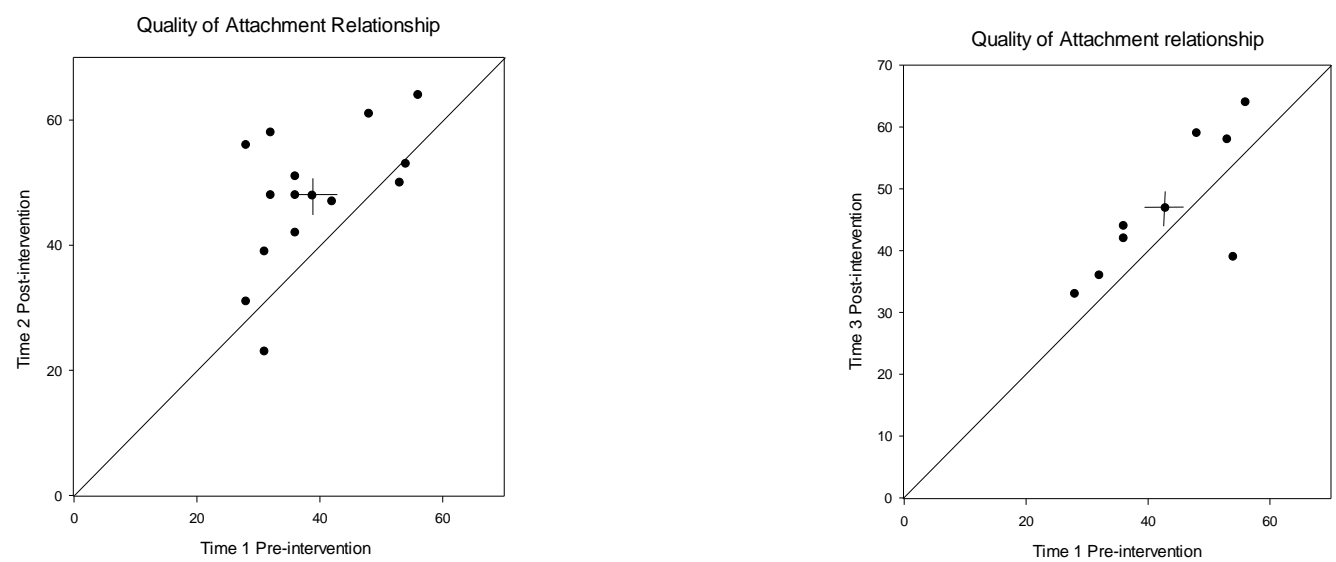

Figure 18. Modified Brinley plots showing pre-intervention scores for the Quality of Attachment Relationship Questionnaire on the $\mathrm{X}$ axis and post-intervention and follow-up scores on the $\mathrm{Y}$ axis.

Depression, Anxiety and Stress Scale (DASS). Chronbach's alphas ranged from $\alpha=$ .71 for anxiety at post-test to $\alpha=0.92$ for depression and stress subscales at six month 
follow-up. The mean parent DASS Stress scores decreased slightly ( $S D=0.03$ ) between preand post-group but markedly increased at six month follow-up $(S D=0.64)$. Using the clinical cut-offs provided in the DASS manual (Lovibond \& Lovibond, 1996), six participants $(50 \%)$ scored over the clinical cut-off for depression at pre-intervention, eight participants (67\%) scored over the clinical cut-off at post-intervention and six participants (67\%) at six month follow-up. For anxiety, seven participants (58\%) scored over the clinical cut-off at pre-intervention and this dropped to five participants $(41.67 \%)$ at post-intervention and five participants (56\%) at six month follow-up. For stress, seven participants (58\%) were over the clinical cut-off at pre-intervention and this dropped to five participants (42\%) at post-intervention and five participants $(56 \%)$ at six month follow-up.

Using UK norms for the short form of the DASS (Henry \& Crawford, 2011) to calculate the RCI, $8 \%$ of participant's scores improved pre-to post-intervention, $75 \%$ did not change, and $17 \%$ worsened. From pre-intervention to six month follow-up $12.5 \%$ of participants' scores had reliably improved, $25 \%$ had reliably deteriorated and $62.5 \%$ showed no reliable change. This finding suggests that the intervention did not have a significant effect on parent's mental health/well-being and may have been deleterious for some foster parents. The magnitude of the increase in follow-up score was largely due to one participant's score almost doubling. A safety check conducted with this participant indicated this was a transitory state and a referral was not necessary. Two foster parents also volunteered that their increased scores were related to life problems rather than the foster child.
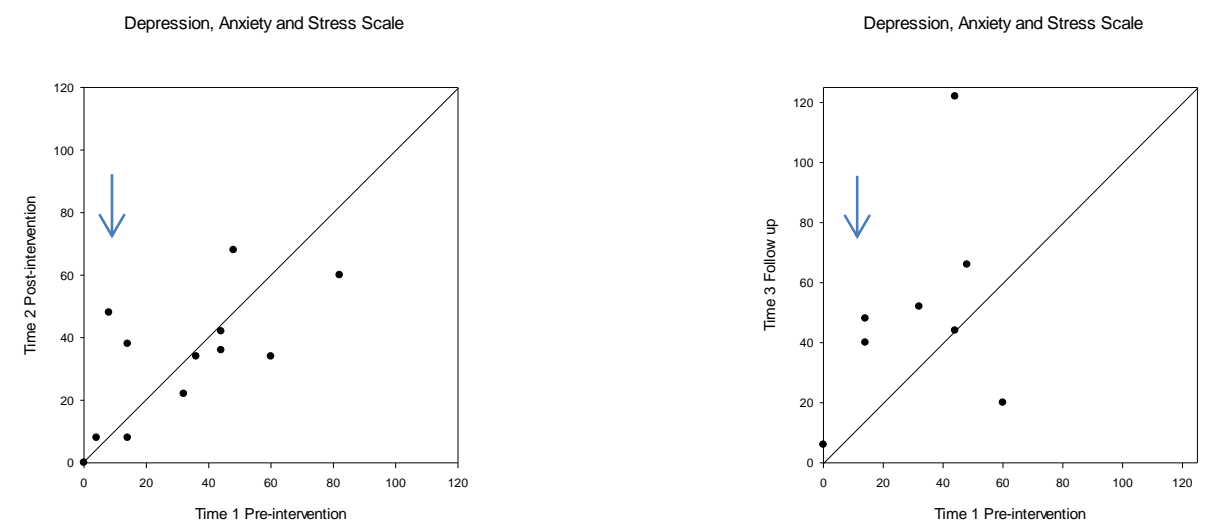

Figure 19. Modified Brinley plots showing pre-intervention scores for the Depression, Anxiety and Stress Scale on the $\mathrm{X}$ axis and time 2 and time 3 scores on the $\mathrm{Y}$ axis. 
Baseline comparisons. Of the 15 participants who completed the group, six participants had a two week baseline, four participants had a three week baseline and three participants had a four week baseline. One participant did only one week of baseline

Baseline comparisons. Of the 15 participants who completed the group, six participants had a two week baseline, four participants had a three week baseline and three participants had a four week baseline. One participant did only one week of baseline recording due to an administrative error and two participants did not continue weekly recordings. Modified Brinley Plots show that during baseline recording $(\mathrm{N}=13)$, the child's daily problem behaviour scores reduced between week 1 and 2 for eight children, stayed the same for one child and increased for five children. Between the second and third weeks of baseline $(N=7)$, four scores decreased, one stayed the same and two increased. Between the third and fourth week $(N=3)$ two scores increased and one stayed the same. The means for each consecutive baseline week were 11.67, 10.35, 10.07 and 17.67 and these means were not significantly different (week 1 to week $2, t=.76, d f=12, p=.46$; week 1 to week $3, t=$ $.00, d f=6, \mathrm{p}=1.00$; week 2 to week $3 t=.50, d f=6, p=.64$; week 3 to week $4 t=-.10, d f=$ $2, p=.93$ ). These results suggest there was no systematic pattern of increases or decreases, despite several participants having baselines with downward trends.

Table 21 Length of baseline for each group

\begin{tabular}{ll}
\hline Group & Baseline length \\
\hline Group 1 & 2 weeks \\
Group 3 & 2 weeks \\
Group 2 & 3 weeks \\
Group 4 & 4 weeks \\
\hline
\end{tabular}
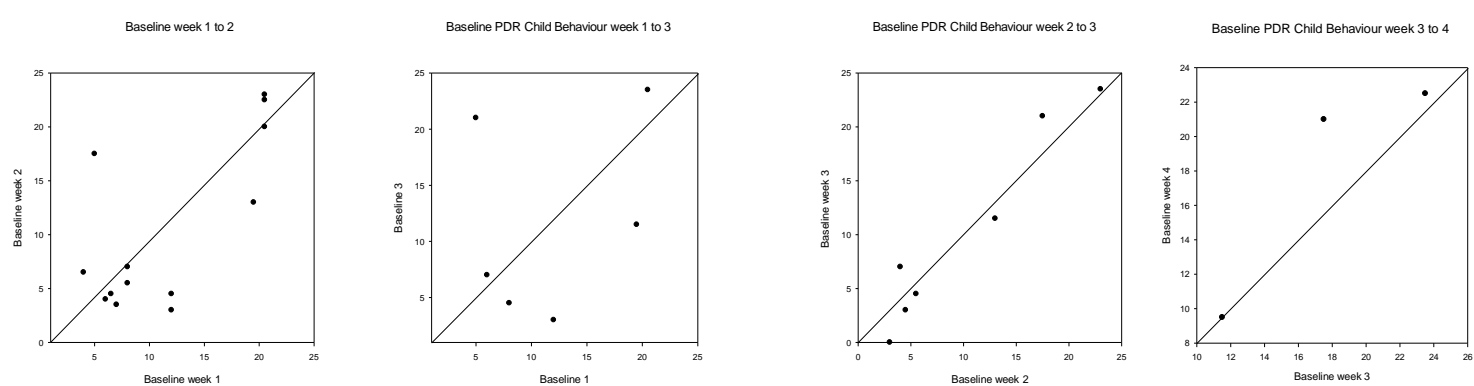

Figure 20. Modified Brinley plots comparing week 1, 2, 3 and 4 of Parent Daily Report: Child Behaviour scores. 


\section{Continuous measures}

Parent Daily Report - child behaviour. Parents completed the PDR on two consecutive days per week throughout the baseline and treatment phases and at six month follow-up.

Table 22 Percentage of Treatment Scores Exceeding the Median of the Baseline for the First and Second Halves of the Treatment Phase and Overall

\begin{tabular}{llll}
\hline & PEM $1^{\text {st } 1 / 2}$ & PEM $^{\text {nd }} \frac{1}{2} 2$ & PEM Total \\
\hline Participant 1 & $78 \%$ & $81.82 \%$ & $80 \%$ \\
Participant 2 & $40 \%$ & $66.67 \%$ & $52.63 \%$ \\
Participant 3 & $100 \%$ & $100 \%$ & $100 \%$ \\
Participant 4 & $81.82 \%$ & $69.23 \%$ & $75 \%$ \\
Participant 5 & $90.91 \%$ & $76.92 \%$ & $83.33 \%$ \\
Participant 6 & $18.18 \%$ & $84.62 \%$ & $54.17 \%$ \\
Participant 7 & $90.91 \%$ & $76.92 \%$ & $82.61 \%$ \\
Participant 9 & $87.5 \%$ & $100 \%$ & $93.75 \%$ \\
Participant 10 & $28.57 \%$ & 0 & $18.18 \%$ \\
Participant 12 & $75 \%$ & $100 \%$ & 83.33 \\
Participant 13 & 62.5 & $70 \%$ & $66.67 \%$ \\
Participant 14 & $40 \%$ & $60 \%$ & $52 \%$ \\
Participant 15 & $86.7 \%$ & $100 \%$ & $93.1 \%$ \\
\hline Mean & 67.70 & 75.86 & 71.91 \\
\hline
\end{tabular}

Graphs of the baseline, treatment and follow-up phases are shown in Figure 14. The graphs were examined for the stability of the baseline phase, with an absence of slope and low variability indicating a stable baseline. One baseline was over the $50 \%$ accepted level of variability (Kazdin, 2001) with 53\% variability and an upward slope (Participant 15). The variability of all other baselines was under the recommended 50\%. Regarding the direction of the slopes, the baselines of five participants showed an upward slope, six participants had baselines with a downward slope and two participants had no systematic trend.

A visual inspection of the graphs for treatment effects was difficult due to the fluctuating scores over time. There were several external reasons for fluctuations such as the death of a foster parent in one agency (affecting eight of the participants), school holidays and children having access visits with their birth family. Using the Percentage Exceeding the Median Effect Size index (Ma, 2006), an average of $72 \%$ of data points exceeded the median of the baseline in the treatment phase, with little difference between the first and second halves. Seven participants had $80 \%$ of the treatment PDRs exceeding the median of the 
baseline, five had between 50 and $80 \%$ of the treatment data points exceeding the median of the baseline and one participant had under $20 \%$ exceeding the baseline median.

[Figure 22 follows on next page] 


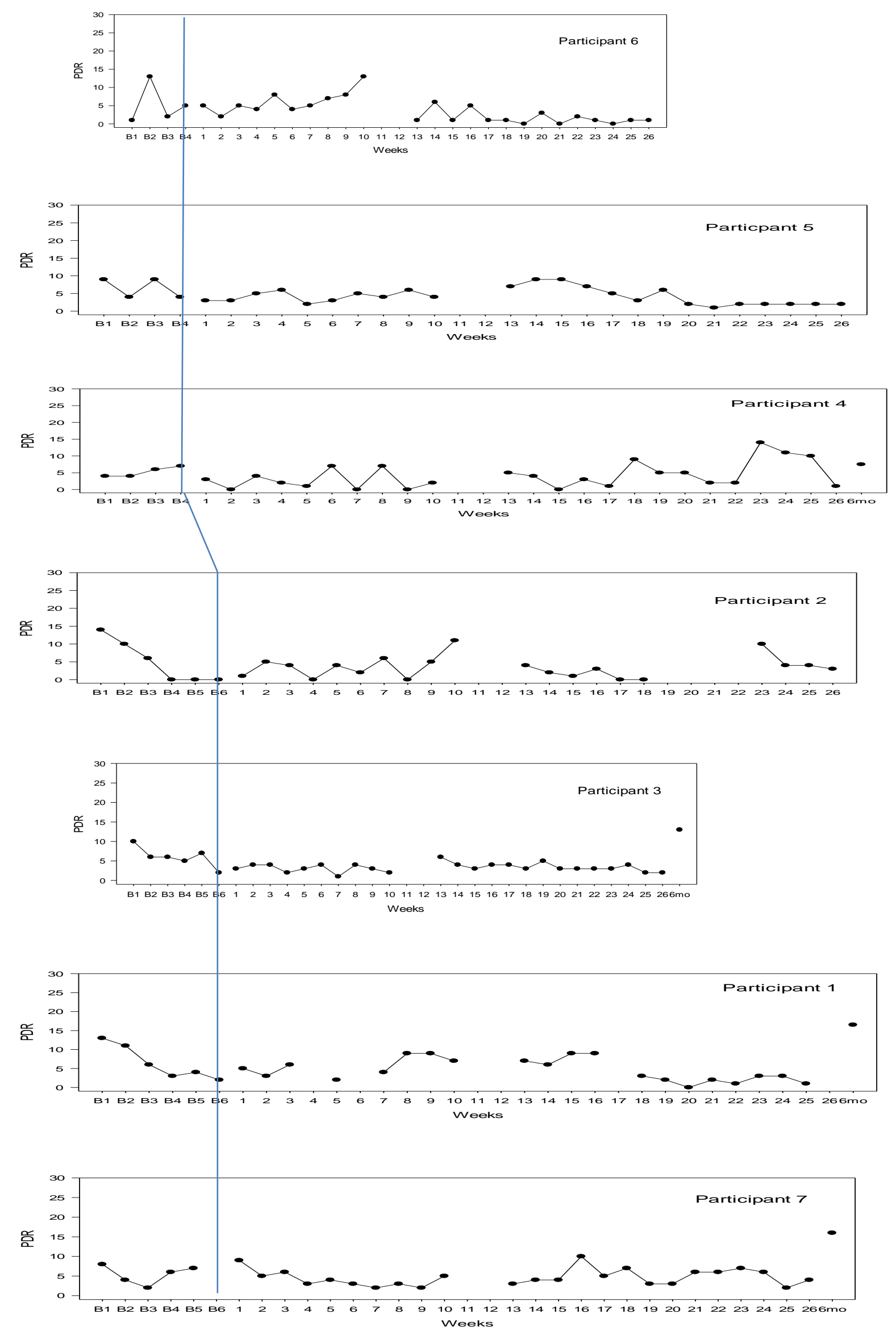




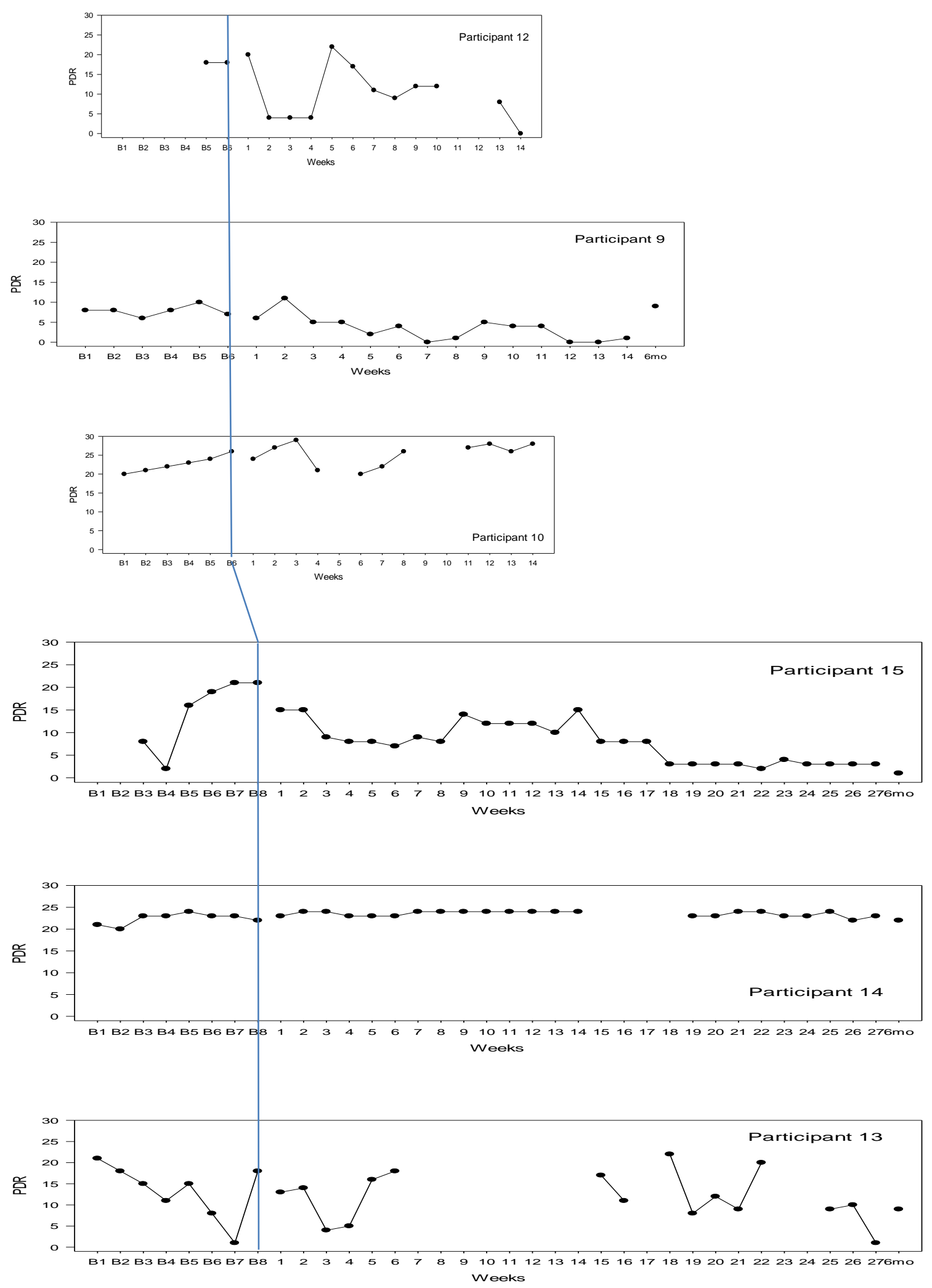

Figure 21. Multiple-baseline across participants graphs for each participant for baseline, treatment phase and follow-up for the Parent Daily Report: Child behaviour.

Note. The letter B indicates baseline period, as does the continuous line. 
Outcome Rating Scale. Examining the graphs for variability, one baseline showed $58 \%$ variability, placing it above the recommended level of 50\%, with an upward slope (Participant 13). Eight participants had an upward sloping baseline (in the direction of improvement before the treatment phase) and four had a downward slope. There was less fluctuation in the Outcome Rating Scale scores over the treatment phase, compared to the Parent Daily Report: Child behaviour, probably due to their global nature and being measured only once a week (see Figure 15).

Using the "Percentage exceeding the median" index, an average of $52 \%$ of data points exceeded the median of the baseline in the treatment phase. Four participants had $80 \%$ of the treatment PDRs exceeding the median of the baseline, one had between 50 and $80 \%$ of the treatment data points exceeding the median of the baseline and five participants had under $40 \%$ exceeding the baseline median.

[Figure 23 follows on next page] 


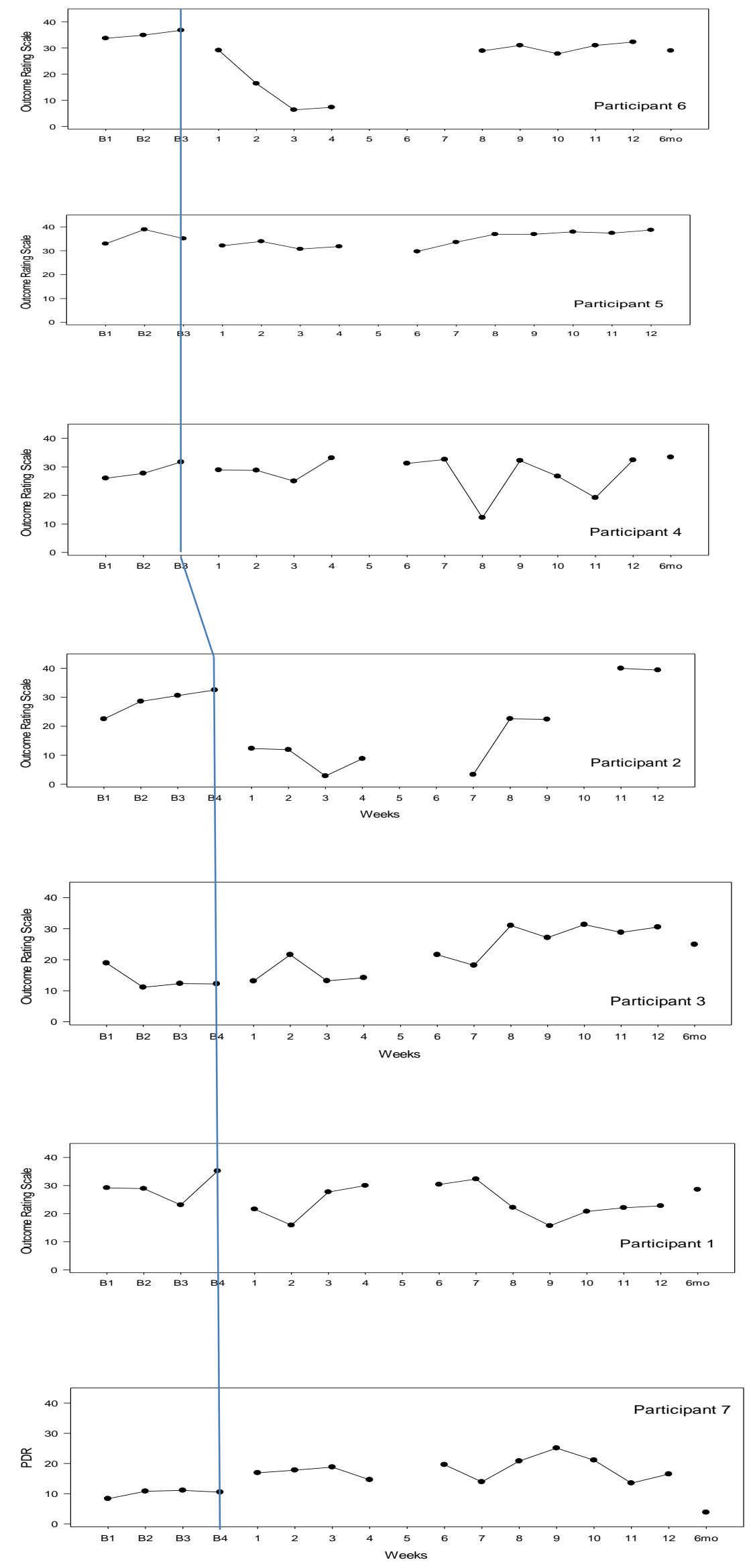




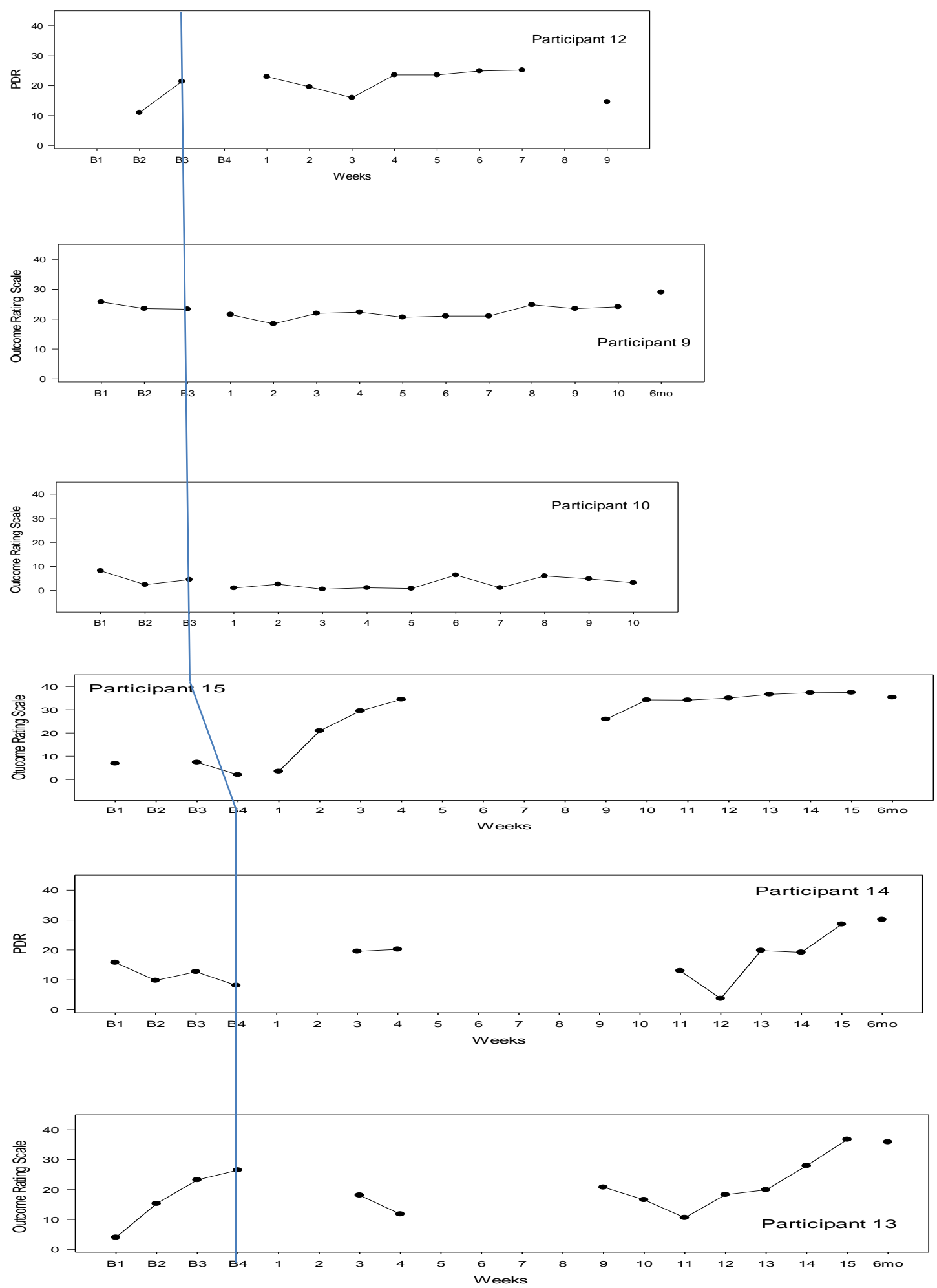

Figure 22. Multiple-baseline across participants time-series data for Outcome Rating Scale. Note. The letter B indicates baseline period, as does the continuous line. 


\section{Discussion}

In this study a newly developed training programme featuring the RLF, which is aimed at improving foster parent's understanding of their foster child, was delivered to five groups of foster parents from three different agencies. The first hypothesis was that at the level of parent's mental representations, the participants would show increases in mind-mindedness and would increase their empathic and reflective comments when asked about a time the child showed difficult behaviour or got into trouble. The second hypothesis was that there would be concomitant changes in the foster parents' well-being and child behaviour problems and attachment. Neither hypothesis was fully supported and mixed results made firm conclusions difficult to draw.

It should be noted that participants were drawn from services for a) hard to place adolescents with histories of severe maltreatment and multiple placements, b) a semiresidential service where parents cared for up to 9 children, with domestic help and social workers on call, and c) participants with children attending a child and adolescent mental health service. These participants are likely to have more severe problems than the wider population of foster parents and children. Compared to the sample of participants in the first study, participants in this study had four times the rate of clinical depression (10\% in study one versus $40 \%$ in the current study) and the children and young people in this study were older and had a mean SDQ score in the clinical range $(M=19)$ compared to the children in study one, whose mean was in the borderline range $(M=15)$.

\section{Mental Representation Measures}

The parent representational measures were mind-mindedness, positive mindmindedness and negative mind-mindedness, reflective comments about a behavioural incident and parent attribution ratings. Parent mind-mindedness significantly increased after the intervention for the pilot group but not for the main sample. This contrasting finding may be explained by the mean mind-mindedness score for the pilot group being considerably lower than that of the main sample, whose mean was close to that of a community sample (Lok \& McMahon, 2006). There may, then, have been a form of ceiling effect operating to limit improvement in this group. At an individual level, there was no discernible shift in mind-mindedness, positive or negative mind-mindedness in the direction of improvement at post-intervention. At six month follow-up, however, the modified Brinley Plots suggest a move in the direction of improvement for all three aspects of mind-mindedness. 
In addition, parents showed a statistically significant increase in positive mind-related comments at six month follow-up. This may be important clinically as research with community samples has found associations between positive mind-mindedness and lower parent stress and hostility and higher observed sensitivity to the child (Demers et al., $2010 \mathrm{~b}$; McMahon \& Meins, 2012). Demers et al. (2010b) also found positive mind-mindedness was related to the mother's state of mind regarding their past attachment experiences and perceiving the child as less difficult.

There were no significant differences between the proportion of reflective comments parents made about a behavioural incident at pre- and post-intervention or six month followup. On an individual level, however, the modified Brinley plots showed that around two thirds of the parents made more reflective and empathic comments at both post-intervention and six month follow-up. A third representational measure of willingness to attribute a behavioural incident to a range of causes showed no significant difference at pre- and postintervention or six month follow-up for either the pilot or the main sample, although there was a trend towards improvement from pre- to post-intervention in the main sample. Again, on an individual level, a majority of both samples showed increases in this measure and in the main sample, the means for post-intervention and six month follow-up were in the direction of improvement. There is a methodological problem with the behavioural incident measure as the behavioural incidents the participants were discussing changed at each time point and the measures may have reflected changes in the incident rather than the parent's attributions relating to them.

There are several possible explanations for these mixed results. One explanation emerges in the research of Meins and colleagues, who have found that mother's mindmindedness is not related to factors such as socio-economic status, education level, general cognitive ability or levels of depression (McMahon \& Meins, 2012; Meins, Fernyhough, Arnott, Leekam, \& Turner, 2011). Following on from this, Meins, et al., 2014) conducted four studies to tease out whether mind-mindedness was a relational or trait-like quality and concluded that mind-mindedness is “... a facet of individuals' representations of people with whom they have close relationships". Thus, it is person-situation-interaction dependent, rather than a trait-like characteristic. This may explain why mind-mindedness appeared to shift in the direction of improvement at six month follow-up rather than post-intervention. It may be a quality which takes time to change within a relationship context, compared to the rapidity of changes expected in other targets of interventions such as dysfunctional thoughts in cognitive behaviour therapy. In addition, no studies have shown that mind-mindedness 
can be changed by a brief intervention as the only study which has used it as an outcome measure did not report a significant difference between mind-mindedness at pre- or postintervention or follow-up (Gurney-Smith et al., 2010).

Another possible explanation for these mixed results may be that mind-mindedness may not capture the main target of the intervention, which was parents' understanding of child behaviour. If this was the case, we would not expect change on the interview measure of reflection and attribution ratings. Unfortunately the mixed results do not clarify this point. The reflective comments scores were not statistically significantly different at postintervention and six month follow-up but two thirds of the main sample showed change in the desired direction at both time points. The attribution ratings showed a similar pattern with a trend towards significance from pre- to post-intervention for the main sample. It is possible that for all three measures, the individual results reflected change in the direction of improvement which was not detected in the group results, due to the small numbers for group statistical techniques and the lack of a control group.

Taken together these results suggest that the intervention may have had a small effect on parents' representations of their foster children but to confirm this tentative conclusion would require a more controlled research design, such as a randomised controlled trial.

\section{Child behaviour problems}

Child behaviour problems showed a significant decrease after the intervention but this was not maintained at six month follow-up. Child daily problem behaviours decreased by almost four daily problem behaviours. The mean score remained in the placement disruption risk range, however, as in this older sample there was a very high level of daily problem behaviours compared to other younger samples (e.g., Hurlburt et al., 2010). These higher scores may be due to adolescents being included in the current sample or to a difference in the way the measure was administered. Chamberlain and colleagues administered the PDR by phone (Chamberlain \& Reid, 1987), whereas participants in this study were given a written checklist, giving parents more time to think about their answer and no possibility of a reminder that the parent should answer just for that day, as may occur in a phone call.

Half of the participants showed statistically reliable change on the PDR daily problem behaviour measure and seven participants also moved from the risk to the non-risk range for placement disruption, giving more weight to the possibility that the decreases in child daily problem behaviour were reliable and clinically significant. However, these results were not 
maintained at six months follow-up and there was a slight increase in the number of daily problem behaviours of just over one problem at follow-up.

A reduction of almost four daily problem behaviours is likely to have some clinical importance as behaviour problems are associated with placement disruption and the child having six or more daily problem behaviours has been found to predict placement breakdown (Chamberlain et al.; 2006: Oosterman et al., 2007). In addition, many studies of treatment effectiveness in foster care either do not measure behaviour change or have not found significant results, even when the intervention targets externalising behaviour (Leve et al., 2010; Linares et al., 2006; Macdonald \&Turner, 2005). The mean decrease of four problem behaviours is larger than the decreases typically found with the PDR such as observed in Chamberlain et al. (2008), who found an average decrease of 1.5 in child daily problem behaviours in a large sample of children whose foster parents completed the KEEP intervention.

In relation to overall emotional and behaviour problems, the findings were similar. SDQ scores decreased significantly from pre- to post-intervention, with a medium effect size and six participants moved from on or over the clinical cut-off to under the clinical cut-off. However, only a minority of the group had clinically significant change and reliable improvement, creating the possibility that change for some participants was due to the reliability of the test rather than actual change. The significant decrease in SDQ scores was not maintained at six month follow-up but remained below the pre-test scores and four participants (44\%) remained below the clinical cut off.

There are several possible explanations for decreases in these two behavioural measures not being maintained at follow-up. It is possible that parents were unable to continue with the techniques they began using in the group, as the RLF is relatively complex and parents may not have been able to apply and modify it without extra support. This may have lead to a lack of generalisation of the use of the techniques with new problem behaviours as they arose. It is also possible that parents may have needed more support to maintain the change and someone who was able to help them apply the learning and to reflect on the child's difficulties.

\section{Other child measures}

There were significant improvements from pre- to post-intervention on the Quality of Attachment Relationship Questionnaire and, while the mean follow-up score remained above the baseline mean, the difference between pre-intervention and six month follow-up was not 
significant. The only other study in which this measure was used reported a significant difference between the intervention and control group on this measure (Briskman et al., 2012). Differences found on this measure indicate an improvement in children's ability to access the foster parent for help and support and the foster parent's view of their ability to give this support.

There was also a significant improvement in carer-defined problem scores, which almost halved. Briskman et al. (2012) have regarded significant decreases on the carerdefined problem scale as an indication of the effectiveness of the intervention they were evaluating, but it is not possible to conclude this without ascertaining whether other problems have become of increased concern. There were also no follow-up data for this measure.

The Outcome Rating Scale showed a significant improvement at post-intervention and was the only measure which remained significantly improved at six month follow-up. The effect size was moderate at pre-intervention and large at follow-up. Over two thirds of the sample scored in the clinical range at pre-intervention but this was reduced to $40 \%$ at postintervention and less than one quarter at follow-up. However, due to a large standard error of the mean, change was only statistically reliable for a minority (26\%) and although no participants' scores deteriorated the majority were within the range of measurement error.

\section{Parent well-being measures}

The only parent well-being measure to show a significant decrease was PDR daily stress, with a large effect size. As the measure is derived by asking the parent to rate the stressfulness of the child's daily problem behaviours, this improvement was also not maintained and the mean score rose slightly at six month follow-up. Several of the foster parent's scores on the DASS deteriorated after the intervention and at six month follow-up. From pre- to post-intervention two participants' scores reliably deteriorated and at follow-up four participants' scores had deteriorated, one markedly so. Two of these participants spontaneously told researchers that their increase in depression, anxiety and stress was due to other stresses in their lives.

This lack of improvement in DASS scores and marked deterioration in four participants' scores at six month follow-up raises the possibility that the intervention had a deleterious effect on parent well-being. One explanation for this is that the RLF may have a negative effect by bringing attention to the adverse experiences the foster child had experienced and may have added to the foster parent's feeling of burden (Murray et al., 2011). Against this, however, caregiver strain did not increase over the intervention and 
follow-up periods. Although the mean total score for caregiver strain did not significantly decrease from pre- to post-intervention or six month follow-up, the mean score at follow-up remained below the pre-intervention level. This result suggests careful modification of the programme is required to address foster parent well being in a more systematic way, if further outcome evaluation of the programme is carried out.

\section{Continuous measures}

A multiple-baseline across participants design was used to evaluate the effectiveness of the intervention so that it could be inferred that observed changes were the result of the intervention, based on observing changes when, and only when, the participants experienced the intervention. Downward sloping baselines for the majority of participants and variable scores over the treatment phase, however, precluded firm conclusions from being drawn from the time-series data. Nevertheless, modified Brinley plots showed no systematic patterns of increase or decrease over each baseline week for the whole group. In addition, over $70 \%$ of the data points in the treatment phase exceeded the median of the baseline for the child's daily problem behaviours and just over half for the child's overall functioning, which suggests improvements occurred over the treatment phase, at least for child daily problem behaviours.

\section{Overall effectiveness of the intervention}

This study used a range of statistical techniques and idiographic methods to measure change in a preliminary intervention study with a small sample and no control group. Discrepancies in results for different indicators make firm conclusions difficult. Comparing group data, the intervention had significant positive effects for child daily problem behaviour, parent daily stress, overall emotional and behavioural problems, carer-defined problems, the quality of the attachment relationship and the child's overall functioning. However, only improvements in the child's overall functioning were maintained at a significant level at six month follow-up. There were no significant increases in mind-mindedness or reflective comments after the intervention but there was a trend to higher attributions after the intervention and positive mind-mindedness significantly increased at six month follow-up. At an individual level, modified Brinley plots showed shifts in the direction of improvement for mind-mindedness at follow-up and reflective comments and attribution ratings at postintervention and follow-up. 
In terms of the proportion of participants who reliably changed or moved closer to the mean of the normative population, only a minority of participants (8\% to $27 \%)$ scored outside the range of measurement error on three of the measures for which this index was calculated (PDR: Parent Stress, the SDQ and the Outcome Rating Scale) although 54\% of the participants reported reliable change on the PDR: Child Behaviour measure, with two thirds scoring in the non-risk range. Although only a third of the participants showed clinically significant decreases in the child's overall emotional and behaviour problems, over half of the participants reported improved overall child functioning at post-intervention and almost four fifths at six month follow-up. Barkley et al. (2001) note, however, that this index of clinical change may inflate the extent of clinical change as it includes participants who were not in the clinical range at pre-intervention. They argue that improvements in functioning after an intervention may be driven by a small number of participants and that researchers who do not view their data ideopathically and calculate these more conservative statistics may overestimate the level of change.

While only one measure remained significantly improved at six month follow-up, although increases in positive mind-mindedness became statistically significant, measures remained below baseline levels except for PDR: Child Behaviour and PDR: Parent Stress which rose slightly at follow-up and the DASS, which rose moderately but not significantly. While this lack of maintenance of gains at six month follow-up might possibly be remedied by offering foster parents more support in implementing and generalising the results, deterioration in a minority of foster parent's mental health/well-being suggest that the intervention requires modification if further evaluation were to be undertaken.

Many studies using a brief group training format for foster parents have not found a significant decrease in children's overall emotional and behaviour problems (Gurney-Smith et al., 2010; Linares at al., 2006; Turner et al., 2007) and it is possible that this group format is not sufficient for the complex problems foster children face. Given that the current intervention produced temporary improvements in foster children's behaviour problems of the sort which have been shown to be difficult to change in foster care (Biehal, Ellison, Baker et al., 2011; Wilson, 2006) it may be worthwhile to evaluate the intervention with a sample of younger children in foster care, with less severe problems. However, if this were to be carried out two significant changes would need to be made to the programme. Firstly, the stress management module should be strengthened and placed at the beginning of the training programme, as suggested by Gaviţa et al. (2012), on the basis that many foster parents report that providing foster care is a strain (Murray et al., 2011). It is also possible that the stress 
aspect of foster parenting may need to be addressed to improve foster parents' capacity to implement the intervention strategies. Secondly, more support would need to be provided after the group to help foster parents sustain the changes. The main reason for this is that some of the techniques may need practice and discussion to apply successfully. Another option is that social workers participate with their client in the RLF section of the group so that they are briefed on the method and can subsequently support the foster parent.

These results should also be evaluated in the context of children showing increases in externalising behaviour when they enter foster care and that foster children do not thereafter show improvements in behaviour over time (Biehal, Ellison, Baker et al., 2011; Lawrence et al., 2006; Wilson, 2006). This finding is perplexing in the light of children being able to make secure attachments with foster parents at similar rates to biological children (Altenhofen et al., 2012; Dozier et al, 2001; Joseph, et al., 2013). Moreover, out of 30 studies of treatment outcome included in a recent narrative review (Kinsey \& Schlosser, 2012) only 16 included a child behaviour measure. Of these studies, four did not use an overall measure such as the Child Behaviour Checklist or the Strengths and Difficulties Questionnaire and only used the Parent Daily Report Checklist. The relative invisibility of child behaviour in this research continues to impair attempts to find evidence-based ways to improve outcomes in foster care.

\section{Strengths and Limitations}

The main strengths of the study were the use of a multiple-baseline design in a pilot study, rather than the frequently used pre- post-test design. In addition, idiographic methods allow some experimental control and a detailed picture of improvement and deterioration in participants' scores, which are often missed in group statistics. The study also used stringent tests of statistically reliable change and clinically significant change.

This multiple-baseline design was used to provide some experimental control in a small outcome study, but as improvement occurred during the baseline phase and before the treatment phase for a number of participants, this limited the conclusions which could be drawn. The lack of a control group means that significant differences from pre- to postintervention cannot be solely attributed to the intervention. Other limitations of the study were the use of a small sample, meaning that there were few possible replications of treatment effects, missing time-series data due to visits to birth families or holiday arrangements, and limited follow-up data, due to some of the young people having transitioned to another placement or out of care. It is also possible that the spacing of the 
groups which were held fortnightly, or three weekly if the school holidays intervened, may not have been optimal to create change. In addition, the groups were disrupted for one agency as the tragic death of one of the participants meant a two month gap in the training.

The sample was obtained by agencies requesting the training so foster parents did not volunteer to do the training and may not have perceived the need for it, either for their foster child or themselves. Just under half of the sample was made up of foster parents of adolescents who had been chronically abused, been placed in multiple placements and most of whom were currently in trouble with the law and truanting. This high needs group may not have been the most appropriate group to include in a preliminary study, although positive outcomes with this group provide stronger evidence for effectiveness . It should be noted that the three participants who volunteered for the group at a mental health service maintained their improvements more than participants in other groups. This group had a co-facilitator and an additional three sessions to apply what they had learned in the group and this may have made a difference. This difference in how groups were conducted, however, may also be a threat to the internal validity of the study. Further limitations included that the author both facilitated the groups and coded the interview data, although the transcripts were anonymised and $30 \%$ were double coded with substantial to high agreement, and that several measures were developed for the study and lacked validity data.

\section{Conclusions}

The intervention was rated highly by participants who reported changes in their thinking about their foster child and a subsequent improvement in their interaction with the child. Individual analyses showed the majority of foster parents improved in aspects of their mental representations of their foster child, especially their reflectiveness about an incident of difficult behaviour and positive mind-mindedness. The results show some support for the effectiveness of the intervention in improving children's daily problem behaviours, which were shown to decrease over the time of the intervention, to be statistically reliable for half of the sample and for the majority of participants to be in the non-risk range at post-intervention. These gains were largely lost at six month follow-up, however. There was stronger support for the effectiveness of the intervention for children's overall functioning which was significantly increased at post-intervention and follow-up, with the majority of participants showing clinically significant change, although the results were less impressive for statistical reliability. A lack of improvement and some deterioration in foster parents' well being/mental health suggests caution in further evaluation of the effectiveness of the group. A replication 
may be worthwhile, however, as the intervention is one of few brief, easily implemented psycho-education groups which has shown a decrease in both child daily problem behaviours and overall emotional and behavioural problems, as long as attention is paid to increasing the stress reduction module and follow-up support for participants. 


\section{Chapter Eight—Study 3: An evaluation of a training programme in foster care for practitioners}

\section{Introduction}

The aim of the third study in this thesis is to evaluate a training programme for practitioners who work with foster children, foster parents and their families. The training programme was described in Chapter Seven. The programme was adapted for the learning needs of practitioners and a treatment manual was developed. The focus of the training programme is to teach practitioners attachment theory, the Relational Learning Framework (RLF), emotion coaching, and an application of the functional analysis of behaviour, which takes foster children's unique situation into account.

\section{Training programme evaluation}

Child welfare organisations use training programmes “...to promote best practices and to ensure compliance with the new regulations and procedures..." (Liu \& Smith, 2011). Child welfare topics are often complex and challenging, including such issues as the effects of sexual abuse and staff may require group discussion and support to begin to appreciate this complexity (Conners-Burrow et al., 2013; Liu \& Smith, 2011). The majority of accredited training programmes in social work, counselling and psychotherapy do not include adoption and foster care in their curricula (Weir, Fife, Whiting \& Blazewick, 2008). It is likely that this applies to clinical psychology training as well.

Although attachment theory is regarded as the most common theoretical framework applied to foster care practice, a South African study showed that social workers had limited knowledge and training in this theoretical approach (Lesch, Deist, Booysen, \& Edwards, 2013). Lesch et al. (2013) found that while “...general attachment theory ideas were implicit in our participants' accounts, formal attachment theory knowledge and applications did not seem to explicitly and consciously inform their foster care work." (p. 1108). There is also a lack of training on the impact of trauma on children's behaviour problems, which can lead to the child's experience being misunderstood (Ai et al., 2013; Conners-Burrow et al., 2013).

Evaluation of child welfare training programmes has been guided by the Kirkpatrick model which describes four different levels of outcome (Antle, Barbee, \& Zyl, 2008). These levels include the participants' a) reactions and satisfaction, b) learning, c) behaviour and 
transfer of learning, and d) organisational outcomes (Curry \& Chandler, 1999). The most common outcomes examined in research are immediate utility reactions, such as whether the training was useful, and immediate affective reactions, regarding whether participants liked the training (Antle, et al., 2008). Retention of the training, behavioural demonstration and organisational outcomes have also been researched (Alliger Tannenbaum, Bennett, Traver, \& Shotland, 1997). Predictors of the transfer of learning from training into child welfare practice have been examined using hierarchical regression and structural equation modelling (Antle et al., 2008; Liu \& Smith, 2011). Predictors of transfer of learning have been found to include: a) the individual's motivation to learn, learning readiness, and immediate learning, b) supervisors' openness and valuing of learning, and c) organisational support (Antle et al., 2008; Liu \& Smith, 2011).

\section{Training Outcome Research in the Social Services}

Studies evaluating training programmes in the social services are often methodologically flawed. A meta-analysis of training professionals in foster care was unable to locate any studies which fitted the NICE Centre for Public Health Excellence Methods Manual criteria (Everson-Hock et al., 2011). Most training evaluation research uses prepost-test designs and measures specifically developed for the study. These measures focus on knowledge taught in the programme, and have little validity data (e.g. Christenson \& McMurtry, 2009). Two exceptions to this are studies which carried out confirmatory factor analysis with the questionnaire developed for the study (Antle et al., 2008; Carpenter, Patsios, Szilassy, \& Hackett, 2011).

Studies of training programmes are often small scale, preliminary pilot studies carried out in the initial stages of programme development. It is therefore difficult to employ a randomised control trial, as participant numbers are typically low and a wait-list control may not meet the needs of a workplace. Despite this, some randomised control trials have been carried out (Antle et al., 2008; Patterson, 2004). For example, Patterson (2004) found that participants who completed a child abuse training programme delivered to the police showed significantly higher knowledge and empathy than a comparison group of those who did not attend the training.. Single case designs may be difficult to implement in general child welfare practice due to the lack of a suitable outcome measure which can be tracked over time. Residential programmes are an exception as staff interaction and behaviour management with the young people can be observed over time (e.g. Crosland et al., 2008). 
Most studies use questionnaires to measure the outcome of social services training. The questions are often very generic, such as, "I have been able to transfer the skills learned in training back to my actual job" (Liu \& Smith, 2011). These questionnaires do not provide information about which skills were applied or how they were applied, providing little information to assist programme development . When interviews are employed, content analysis or thematic analysis is used to analyse the data for themes, including participants' reactions to the course and the usefulness of the material (Braun \& Clarke, 2006).

\section{Training in child welfare and foster care}

Very few studies have been undertaken on the quality of training of social workers, therapists and clinical psychologists working with foster children and foster families. Everson-Hock et al. (2011) note that "While numerous training programmes have been developed by individual and private agencies, few have been evaluated and reported on..." (p. 173).

Weak methodology is evident in some studies (e.g. Kessler \& Greene, 1999; Dugmore \& Cocker, 2008). For example, a study investigating the effectiveness of a one day training designed to raise awareness of sexuality issues when working with gay and lesbian foster and adoptive parents used a generic "tickbox" evaluation form, with limited content validity (Dugmore \& Cocker, 2008). Although post-training interviews were conducted, the authors reported only a limited number of comments made by participants e.g., frustration that all material could not be covered due to time limits. This information is arguably of little value to other researchers.

Some studies have found that social services training programmes lack effectiveness. One evaluation study of a training course on the impact of parent mental illness on children and their child protection needs found that participants' knowledge about parent mental illness and its effect on children actually decreased (Carpenter et al., 2011). The study concluded that the trainer's emphasis on de-stigmatisation, and how well parents with mental illness can do with support, may have inadvertently lead participants to de-emphasis the harm children were exposed to. This study is a salutary reminder that training programmes may not effect change in the desired direction and improvement cannot be assumed. Other child welfare studies have also shown no significant change in workers attitudes or practice (Alliger,et al., 1997; Alpert \& Britner, 2005).

Studies using thematic analysis, content analysis and focus groups by definition cannot provide quantitative or behavioural information about the effectiveness of a training 
programme. However, they can provide useful information for the further development of an intervention, especially at the pilot stage. For example, one study used pre-training focus groups to identify the challenges in caring for foster children, for both carers and supervising social workers, and employed post-training focus groups to evaluate the effectiveness of the training (Allen \& Vostanis, 2005). Allen and Vostanis concluded from the participants' feedback that skill training groups may be less effective than providing a "...conceptual framework to understand the challenges presented by children with attachment difficulties..." (p. 79).

Some studies have used more sophisticated methodological approaches in their evaluation of training programmes. For example, one research project examined the effect of training on substance use and adolescent services for child welfare workers. Principle components analysis was used to ascertain what dimensions were being measured, and hierarchical regression was used to identify predictors of transfer of training. The study found that putting the training into practice was influenced by both individual factors such as training motivation, as well as by team and supervisory support (Liu \& Smith, 2011). Positive outcomes were reported in a study examining the training of supervisors to better understand issues for youth transitioning out of care (Atukpawu, Mertinko, Graham, \& Denniston, 2012). Innovations in this study included evaluating learning transfer by comparing documentation of supervisors who had and had not attended the training, surveying case workers about their supervisors' practice and comparing feedback from youth in the year the supervisors did the training, compared to other years. One well designed study used a multiple-baseline design in a residential setting and found the training on positive behaviour change increased the amount of time staff were observed to be interacting with the children and the interactions were also more positive (Crosland et al., 2008).

In summary, the literature on training practitioners in foster care is sparse and while a few recent studies have utilised improved methodology, most studies are preliminary pilot studies with small samples and limited outcome measures.

\section{Aims of the current study}

Working with children in foster care crosses disciplinary boundaries between social work, counselling, psychotherapy and psychology. The aims of this study are a) to train practitioners in the delivery and principles of the Lightbulb training programme, described in Chapter Seven, b) to evaluate whether the RLF is useful in assisting social workers and therapists/psychologists in their work with foster children and foster families and c) to 
evaluate the effectiveness of the training. This study extends the findings of the second study described in Chapter Seven, by evaluating the effectiveness of the Lightbulb training programme for practitioners, with the aim of both teaching them to deliver the group and also incorporating the methods into their clinical practice.

As part of the training, the RLF was completed for a number of children on the practitioners' caseloads (at least one per team). If the training programme is successful, social workers and therapists will have a coherent method, with preliminary empirical findings, for developing treatment or care plans for foster children they are working with. Foster children often have multiple, complex problems and care plans may not focus on psychological factors such as the aim of helping the child to trust others, learning that relationships can last and understanding what a family is. This model brings psychological and relational concepts into prominence and the final step is to turn that into an action plan, along with verbal messages that will be helpful for the child's self-understanding.

The study hypotheses were that the training programme would increase foster care practitioners' mind-mindedness, reflection and empathy and that they would report positive effects from using the training material with foster parents and children.

\section{Method}

Procedure. This study employs a pre- post-test design and quantitative and qualitative methods to investigate the impact of training 16 social workers, therapists, and psychologists in the use of the RLF. The practitioners participated in an audiotaped interview before and after the training programme (one tape did not record due to technical problems). There were three parts to the interview. The first part consisted of questions about the participant and their work: a) demographic questions, b) questions about the participant's position and role in their agency; c) length of service and satisfaction with role; d) theory and knowledge which underpins their work (see Appendix E). The second part of the interview consisted of the administration of Mind-mindedness interview and the third part consisted of the scenarios for the Articulated Thoughts in Simulated Situations paradigm (Zanov \& Davison, 2010). In this paradigm, a scenario is read to the practitioner about a difficult foster parent-child interaction and the scenario is paused while the practitioner is asked "What thoughts are running through your mind?" After the scenario is complete, the practitioner is asked to say what they would say or do if they were working with a child with a specific problem. 
The practitioners also kept a diary in which they reported the answers to three questions about their response to the training in the weeks between the training sessions, at the end of the training and at three month follow-up. Participants completed a training evaluation at the end of the training programme and at three month follow-up. Participants also completed the Human Services Training Effectiveness Postcard at three month follow-up. This is a standardised 5 item questionnaire about training satisfaction.

Participants. Two training groups using the Lightbulb programme were conducted in a similar way to that described in Chapter Seven. The five participants in the Group A group came from two services. Four social workers attended from a community agency which runs a foster care programme for hard to place adolescents with histories of severe maltreatment, multiple placements, residential care, and contact with Police and Youth Court. One social worker attended from a residential programme for youths with sexually harmful behaviour. Group B was made up of 11 participants from different services: a) six therapists or psychologists from an agency which works with children and adolescents with sexually harmful behaviour, many of whom are in residential or foster care, b) one social worker from a residence for adolescents with sexually harmful behaviour, c) two caregiver liaison social workers from child welfare services, d) a social worker/therapist from a Child and Adolescent Mental Health Service and e) a psychologist in private practice. The participants consisted of 14 women and one man, and the ethnicities were NZ European (8), Other European (6), Samoan (1), and Māori (1).

\section{Measures}

Demographic interview. This interview was conducted by phone and included questions about the practitioner's qualifications, role in their workplace, theoretical models and therapeutic approaches used in their work, difficulties encountered in their work and their supervision arrangements.

Mind-mindedness. The Mind-mindedness Interview was described in Chapter Four. The difference in this study was that participants were not in the role of a parent and were asked to describe a child in foster care whom they were currently working with. If the participant asked whether they should talk about the child's history or their clinical symptoms, the participant was invited to focus on "What is the child like in themselves?" This question is additional to the usual administration of the mind-mindedness interview. Thirty per cent of the verbatim transcripts were double coded which produced a kappa of $\kappa=$ 0.85 . 
Articulated Thoughts in Simulated Situations. This paradigm was developed to elicit and assess participants' thoughts and has been used in a variety of clinical studies (Zanov \& Davison, 2010). This "think aloud" paradigm can be tailored to the needs of the particular study being conducted. Participants usually voice their thoughts into a recording device but in this study they articulated their thoughts to the interviewer over the phone. The ATSS has been used in over 68 studies, typically evaluating interventions for anger, anxiety and depression and asking participants to think aloud about appropriate scenarios. The method has the advantages of being very specific but allowing an immediate and free response to the stimulus provided. It is difficult to measure test re-test reliability in such specific conditions but two studies have shown comparable results with the same scenarios (Zanov \& Davison, 2010). Construct, criterion and content validity have each been demonstrated in studies showing, for example, that ATSS verbalisations are related to observed anxiety in public speaking and ATSS self-efficacy evaluations were related to success in smoking treatment at three month follow-up (Zanov \& Davison, 2010).

The participants were presented with two of six scenarios before the training and two after the training (in random order). The scenarios were developed by the author and covered common difficulties arising in foster care (see Appendix C). The scenarios were evaluated for realism and level of difficulty by four psychologists familiar with working with children and adolescents. Parts of the scenarios evaluated as easier or more difficult for practitioners to work with were then amended to make the scenarios as comparable as possible. The interviewer read each scenario and stopped at three time-points (see Appendix C). Participants were asked:

1. "What is running through your mind when you hear that?"

2. "What would you advise the foster parent to do or say in the situation?"

3. "What would you do or say if a child who [...] was on your case load?"

The participants then articulated their thoughts in response to each section of the scenario, and the prompt questions. The participants' answers were transcribed by a research assistant and a coding scheme was devised by the author to evaluate changes in the participant's empathy and reflectiveness, which were specific targets of the intervention (see Appendix F). Codes included a) empathy statements, b) reflection on the causes of the child's behaviour, both current and past, c) providing a secure base statements and d) putting thoughts into words for the child. The transcripts were then coded by the author and $30 \%$ were coded by a second coder. The level of agreement between coders was calculated, yielding a kappa of $\kappa=0.79$. 
Practitioner diaries. Participants were given a one page diary sheet to complete before the next session answering three questions:

1. What thoughts have you had about the training in your work from over the past month?

2. Did you apply any of the learning to your work?

3. What was the outcome?

The diary transcripts were processed using thematic analysis as described by Braun and Clarke (2006). Firstly, the transcripts were read and re-read and common ideas organised into groups to generate codes. This list of codes was then analysed to examine overarching themes and sub-themes. The criteria for creating themes were that "Data within themes should cohere together meaningfully, while there should be clear and identifiable distinctions between themes" (Braun \& Clarke, 2006, p. 91). A chart was produced detailing how the themes and sub-themes relate to each other. The resulting themes were then defined and refined (see Appendix F). The coding was completed by the author and a research assistant. The level of agreement between the two coders was $84 \%$.

Training evaluation questionnaire. The training evaluation questionnaire was created for the study and consisted of 5 Likert scales about the participants' evaluation of the training. This was the same evaluation form used in the foster parent study, with appropriate adjustments to wording for practitioners. The evaluation was repeated at three month followup.

The questions asked practitioners to rate the usefulness of the programme, whether it changed their thinking about foster children and parents, whether it changed the way they behaved or interacted with foster children and parents, how the foster children and parents responded and whether they would recommend the programme. The Likert scales ranged from 1 (Not at all) to 5 (extremely or a great deal).

Human Services Training Effectiveness Postcard. Three months after the training the participants were sent the Health Services Postcard consisting of five questions about their satisfaction with the training. The questions use a 6 point Likert scale and cover satisfaction with the training, amount of learning, using the knowledge and skills, observing client progress and being a more effective worker as a result of the training. This postcard was been developed for the evaluation of training programmes in the child welfare area that use the Kirkpatrick model. It is designed to be administered at three month follow-up, to allow time for skills to be applied, and to be brief enough for an acceptable return rate. It has 
good test-retest reliability (83\%) and high internal validity $(\alpha=.91$; Curry $\&$ Chandler, 1999).

\section{The Lightbulb training programme for practitioners}

\section{Aims of the training programme}

The aim of this training programme was to assist practitioners to understand the children on their case load by employing the RLF to make connections between the children's adverse experiences, their problems and strengths, and the implications of these for the child's view of themselves and other people. This understanding is then distilled into treatment goals focussed on changing the child's internal working model of themselves, relationships and their future. The RLF essentially provides the practitioner with a way of conceptualising or formulating a child's complex problems.

The group was provided with theoretical knowledge in the following areas related to foster care: a) types of attachment and how secure attachment develops, b) effects of maltreatment and loss, c) emotional communication, d) understanding the difference between support and empathy, e) providing empathy to foster parents and children, f) social learning theory, functional analysis and how to apply it. The functional analysis is specialised to foster care and includes questions about what the child is trying to achieve through the behaviour and what emotion is the behaviour designed to change. In foster care, the answers may include trying to end the placement or be placed back with biological family.

To deliver the training programme with practitioners, the author first presented the training material in the way it would be delivered to foster parents. Information was then given about how to present the material, how to discuss it in the group, how to teach techniques, and answer questions that foster parents often ask. Methods to incorporate the material into clinical practice were also discussed. Group A received the training in 6 half day seminars held fortnightly over 3 months and Group B received 3 one day training seminars over a 3 month period, due to some participants travelling to the course. Practitioners completed a brief diary about how they had used the training in between sessions.

The key information and therapeutic methods the participants were taught were attachment theory, the RLF, the "Iceberg" problem solving exercise, a variant of functional analysis adapted for foster care and emotion coaching (Gottman \& Declaire, 1997). The 
"Iceberg" problem solving exercise allows practitioners to answer foster parents' questions by working through a short exercise.

This exercise consists of asking the group:

1) What is the situation?

2) What is the purpose of the behaviour?

3) What was the child feeling?

4) What was the child thinking?

5) How can you put that into words for the child? A worked example from one of the groups is provided below.

Table 23 Worked example of the "Iceberg exercise" used for problem solving

\begin{tabular}{|l|l|}
\hline Situation & $\begin{array}{l}\text { Child cried, screamed, threw a tantrum over the dentist telling him } \\
\text { he wasn't going to need new braces on his teeth. }\end{array}$ \\
\hline Purpose of behaviour & Communication, attention, expressing overwhelming feelings. \\
\hline Feelings & $\begin{array}{l}\text { Feeling let down, embarrassed, disappointed, sad, frustrated, like } \\
\text { hope had gone, jealousy. }\end{array}$ \\
\hline Thoughts & $\begin{array}{l}\text { My friend has got braces. People won't make a fuss of me like I } \\
\text { thought they were going to. I won't be special. I'm not in control. }\end{array}$ \\
\hline $\begin{array}{l}\text { Putting it into words } \\
\text { (validation and } \\
\text { empathy) }\end{array}$ & $\begin{array}{l}\text { I can see you're upset and disappointed... what is it about? } \\
\text { I know it hasn't turned out like you wanted it to. } \\
\text { If you could get braces, what colour would they be? What would be } \\
\text { good about it? }\end{array}$ \\
\hline
\end{tabular}

\section{Results}

Data analysis. Data were analysed to examine a) whether the training programme was acceptable to the participants, b) whether it increased practitioners' mind-mindedness and empathic and reflective comments about a foster care scenario and c) the practitioners' use of the training material and whether it was effective in their work. First, participants gave feedback on their experience of the intervention, using an evaluation questionnaire created for this purpose and the HSTEP questionnaire. Second, results were considered at the group level using paired sample t-tests (pre- and post-intervention) and effect sizes. Third, the results were considered on an individual level using modified Brinley plots. The Reliable Change Index (RCI) was not used as population norms were not available for either quantitative measure. Fourth, thematic analysis was used to extract information from diary entries about how practitioners used the course material and the effectiveness of this. 
Mind-mindedness. There was no significant difference between participants' mindmindedness scores before and after the training programme (see Table 24). It is noteworthy that in study two, shifts for foster parents did not occur until follow-up and this study did not include a follow-up for mind-mindedness. The valence scores (positive and negative mindmindedness) were also not significantly different from pre- to post-test.

Table 24 Means and Standard Deviations for the Articulated Thoughts in Simulated Situations Interview and Mind-mindedness

\begin{tabular}{|c|c|c|c|}
\hline & Time 1 Mean $(S D)$ & Time 2 Mean $(S D)$ & t-test \\
\hline $\begin{array}{c}\text { ATSS Total } \\
\text { comments }\end{array}$ & $10.26(7.43)$ & $12.33(5.47)$ & $-1.72^{\mathrm{t}}$ \\
\hline ATSS proportion & $0.77(0.43)$ & $1.14(0.58)$ & $-2.53^{*}$ \\
\hline MM & $.35(.14)$ & $.37(.15)$ & -0.25 \\
\hline MM Positive & $.26(.20)$ & $.31(.19)$ & -0.93 \\
\hline MM Negative & $.56(.34)$ & $.45(.27)$ & -0.98 \\
\hline
\end{tabular}

${ }^{\mathrm{t}} \mathrm{p}<.01 ; * \mathrm{p}<.05$ (two tailed); $N=15$

Modified Brinley plots showing pre- and post-test scores are shown in Figure 23. Visual inspection of the Modified Brinley Plot show an approximately equal number of participants improved and deteriorated on all three mind-mindedness measures, indicating there was no treatment effect.
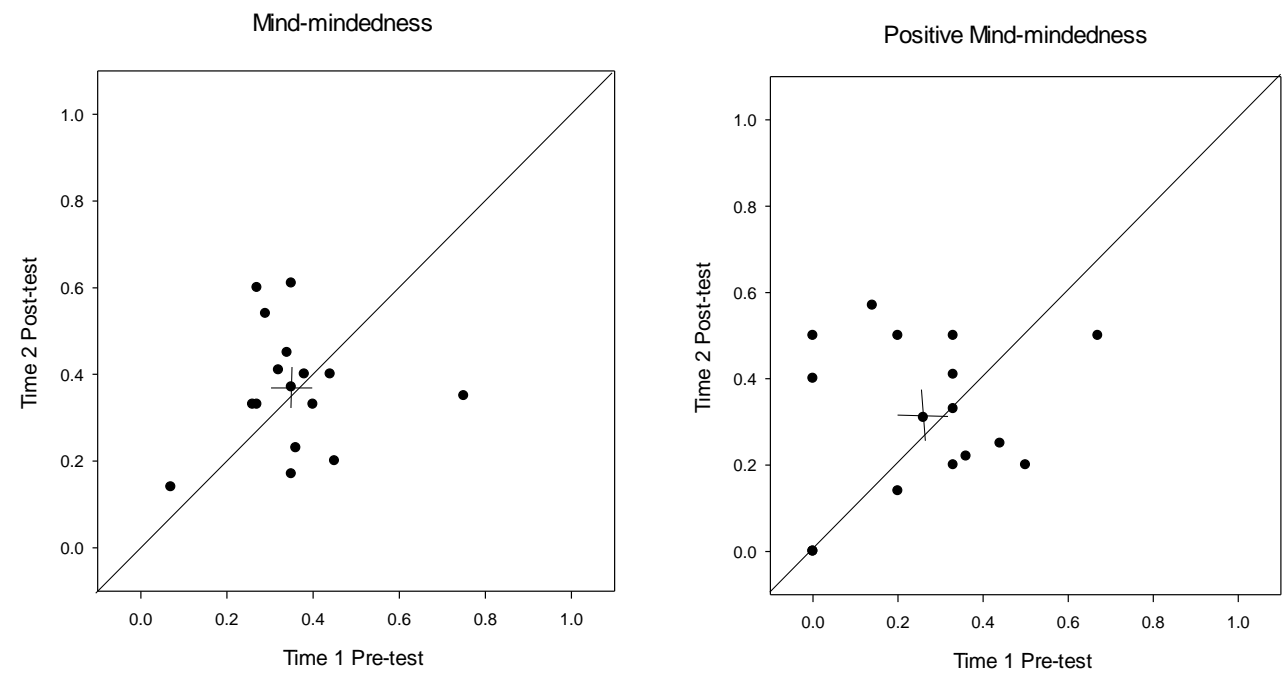


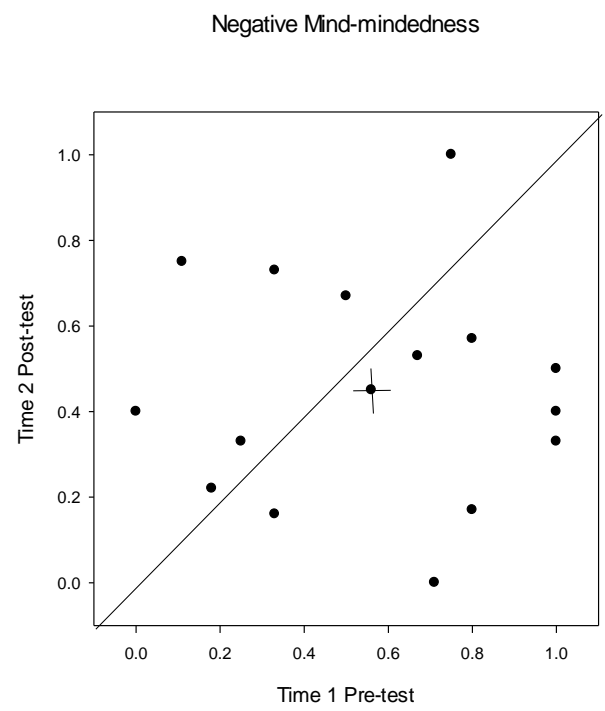

Figure 23. Modified Brinley plots showing pre-intervention scores for the mind-mindedness, positive mind-mindedness and negative mind-mindedness on the $\mathrm{X}$ axis and post-intervention scores on the $\mathrm{Y}$ axis.

Articulated Thoughts in Simulated Situations. The total number of empathic and reflective comments made by participants in response to foster care scenarios increased from 10.27 to 12.33 , which was a $17 \%$ increase $(E S=0.32)$. This was not significant (see Table 24). The proportion of empathic and reflective comments increased from .77 to 1.14 , which was a $68 \%$ increase $(E S=0.51)$. This difference was significant with a small to moderate effect size. This finding suggests that the practitioners increased the proportional amount of empathic and reflective comments after the intervention, with a moderate effect size.

Modified Brinley plots showing pre- and post-test scores are shown in Figure 24. Visual inspection of the modified Brinley Plots shows that the majority of participants had improved scores on both total and proportional empathic and reflective comments. The means for ATSS Total comments and proportional comments both shifted in the direction of improvement. 

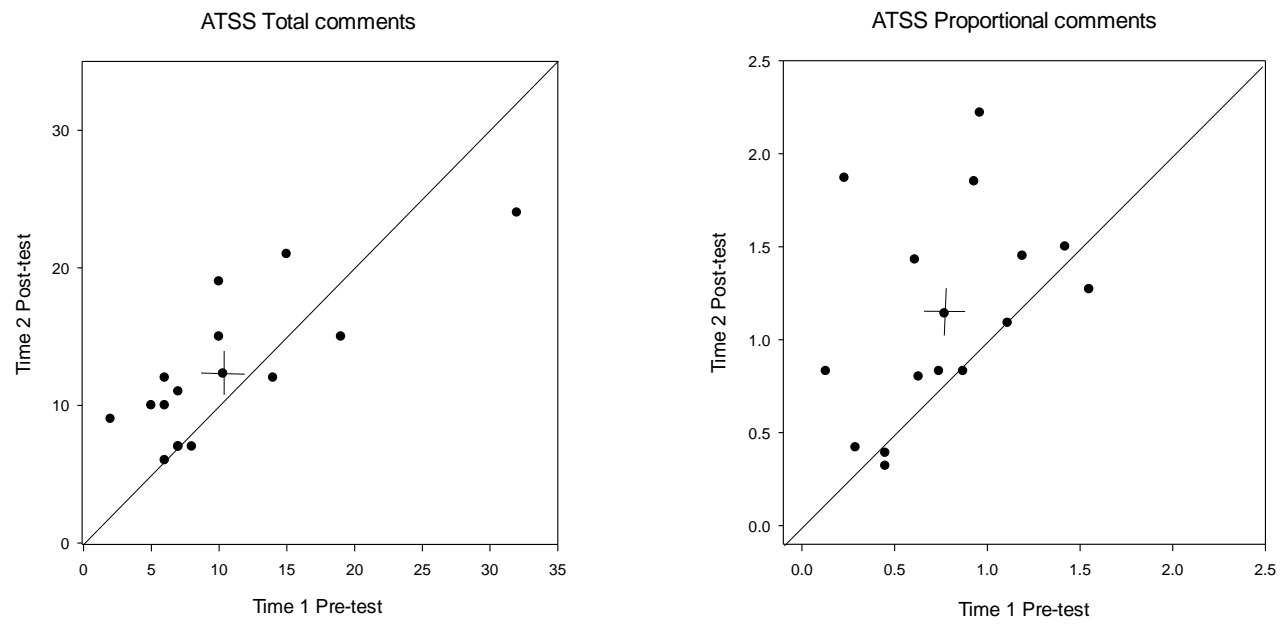

Figure 24. Modified Brinley plots showing pre-intervention scores for the Articulated Thoughts in Simulated Situations paradigm on the $\mathrm{X}$ axis and post-intervention scores on the $\mathrm{Y}$ axis.

Training programme evaluations. The evaluation form designed for this research was completed at post-test and three month follow-up, with overall means of $M=3.95$ at post-test and $M=3.88$ at follow-up. The Human Services Training Effectiveness Postcard is designed to be completed 3 months after the training. The overall mean for this measure was $M=4.34$. The means and standard deviations of each measure are shown in Table 25 and 26.

Table 25 Means and Standard Deviations for the Training Evaluation Form at post-test and three month follow-up)

\begin{tabular}{|l|l|l|l|l|l|}
\hline $\begin{array}{l}\text { Training Evaluation } \\
\text { Form }\end{array}$ & Usefulness & Change thinking & $\begin{array}{l}\text { Change in } \\
\text { interaction }\end{array}$ & $\begin{array}{l}\text { Response of } \\
\text { child/parent }\end{array}$ & $\begin{array}{l}\text { Recommend } \\
\text { to others }\end{array}$ \\
\hline Post-test & $4.4(.63)$ & $3.6(.81)$ & $3.9(.93)$ & $3.1(.70)$ & $4.75(.45)$ \\
\hline Follow-up & $4.3(.67)$ & $3.5(1.18)$ & $3.4(.70)$ & $3.4(1.07)$ & $4.80(.42)$ \\
\hline
\end{tabular}

Table 26 Means and Standard Deviations for the Human Services Training Effectiveness Postcard (HSTEP) at three month follow-up

\begin{tabular}{|l|l|l|l|l|l|}
\hline HSTEP & $\begin{array}{l}\text { Satisfaction } \\
\text { with training }\end{array}$ & $\begin{array}{l}\text { Substantial } \\
\text { Learning }\end{array}$ & $\begin{array}{l}\text { Used skills \& } \\
\text { knowledge }\end{array}$ & $\begin{array}{l}\text { Observed client } \\
\text { progress }\end{array}$ & $\begin{array}{l}\text { More effective } \\
\text { worker }\end{array}$ \\
\hline & $4.8(.38)$ & $4.4(.58)$ & $4.3(.34)$ & $4.0(.62)$ & $4.2(.48)$ \\
\hline
\end{tabular}

HSTEP = Human Services Training Effectiveness Postcard ( three month follow-up).

\section{Thematic analysis}

The purpose of the training diary was to access participants' thinking and use of the training techniques in their work. Open questions were used so that participants would 
record their thoughts without prompting. It was designed to examine whether practitioners used the skills and knowledge in their practice, which aspects of practice the training impacted, whether this impact was positive or negative and how easy or difficult the skills and knowledge were to apply in clinical practice. Transcripts were double coded by the author and a research assistant and inter-rater agreement was $84 \%$.

The questions in the training diary were:

1. What thoughts have you had about the training in your work over the past month?

2. Did you apply any of the learning to your work?

3. What was the outcome?

Overarching themes. The main themes in the participants' diaries were categorised into five themes and a number of sub-themes (see Table 27).

Table 27 Main themes of thematic analysis

Overarching theme Sub-themes

Use of the RLF

a) Use of the RLF in client work

b) Advantages in using the RLF

Applying attachment

theory

Skills and techniques used

a) Understanding the causes of behaviour

in client work

b) Empathy and validation techniques

c) General skills

Difficulties in using the

a) Challenged in implementing the RLF

training material

Positive changes

a) Positive outcomes for foster parents or children from using the RLF

b) Being more mindful, more aware and gaining insight

The Relational Learning Framework. All but one participant reported that they had used the RLF in their work and 28 diary entries mentioned using the framework. The framework was used for a variety of purposes, depending on the role of the participant. Purposes included working with foster parents (7 entries), completing the framework as a team for new referrals (12 entries), supervision (4 entries), clarifying own thinking (4 entries) and training staff ( 2 entries). Two agencies began using the RLF as part of their intake 
procedures, one agency began running a group and one participant delivered the programme with an individual foster parent:

Participant 9: "It was great at creating a new perspective of the child for that foster parent. She was able to understand where the behaviour comes from (emotional needs) and how to respond. Before the training she used to complaint a lot about the kid and thinking his behaviour was with the purpose of annoying her. The behaviour changed over time with change in her responses. This was showed by the weekly reports of the foster parent in regard to the behaviour change."

Participant 4: "The training gave me an easy to apply framework for discussing a child's problem behaviours from an attachment perspective. I felt that it really increased my own compassion for the child and the caregiver was really open to seeing things differently and willing to try anything."

Advantages of the framework. Two thirds of the practitioners mentioned one or more advantages of using the framework (18 diary entries). Some practitioners talked about how the framework helped them to organise their thinking about the child, including considering information more closely and identifying significant information, which may have been overlooked before:

Participant 16: "I've found the relational framework helpful when trying to organise my thoughts to write a report as it helps me to recognise different aspects of the information that has been gathered".

Several practitioners talked about sharing the theoretical information with young people, social workers and teachers:

Participant 7: "They react in a positive way almost instantly to it and they all agree that the information is helpful and accessible. They practice the techniques in home and the feedback I have is that they 'surprisingly' work".

Many participants reported that it was the application of the theory that was helpful, that is, using the RLF to make sense of a large amount of information about the child: 
Participant 15: "I think this model is a compact way to help parents understand their child's behaviour".

Participant 16: "The process has been helpful to summarise the history in one place, to identify trends and to highlight concerns..."

Difficulties implementing the framework. Six participants reported difficulties implementing the framework (11 entries). One participant reported having little time and funding in her consultation role:

Participant 11: "It takes time to get staff to think this way and respond appropriately and in my current role I don't have the time."

Two participants reported finding it difficult to reflect on or implement the training material on their own. This included one participant reporting difficulty in reflecting on the learning in isolation and another participant reported difficulty in completing the RLF in isolation. This RLF was completed with foster parents after the first training day, although participants had been advised to wait until they had more practice. This experience helped the participant to appreciate that the RLF works better in a group, and that she needed practice and supervision. One participant reported that the information in the training programme was too general for a very experienced foster parent. Another participant also mentioned that it was difficult to think empathically under stress and:

Participant 16: “... not wanting to attribute thoughts and feelings to a child”.

Positive changes. All participants reported positive changes in the young people and foster parents they were working with (39 diary entries). These positive changes included foster parents and children learning about emotions, clients being more focused and engaged, improvement in children's behaviour or the child calming down and better joint understanding:

Participant 10: "Positive regard from Mother to Child - Positive response from Childvisibly reduced anxiety and allowed child to come back 'into view". 
Participant 15: "Parents appreciated the practical approach and were willing to look at their own emotional regulation as well. Family really needed time to vent."

Participant 14: "Better understanding. Helps the team around the YP [young person] all understand the situation and develop a plan to support... Model allowed us to best understand which lead to a very open and supportive conversation when first meeting young person."

Emotion, empathy and validation. Two thirds of the participants reported thinking more empathically, helping children with emotion regulation and helping foster parents validate the child's emotions (18 diary entries). Seven participants reported having more empathy, one mentioned having more compassion, five mentioned helping children with emotion regulation and two reported helping foster parents validate the child's emotions. The participants also reported thinking more about the impact of the child's experiences, especially trauma, and the child's perspective:

Participant 5: "I have used the Lightbulb training with social workers on how they can work with the caregivers to use an empathic approach with the child in their care, how to deal with the stress of caring for children not born to them, to think about how abuse and neglect can affect children's behaviour and how important it is for foster parents to try and separate behaviours from the child".

Participant 4: "Continued to work with caregiver around how she can be more emotionally validating. However, child has been amping up his behaviour even more so this has been a challenge. Looked underneath the behaviour and identified the child was anxious because of ....Explored with caregiver how she can respond to that level of distress... Validating feelings builds the connection and strengthens relationship and also fills the gap in their developmental needs".

Participant 11: "I have been thinking more about emotion regulation in young people, and the FPs emotional awareness of these young people and their ability to empathise"... In the process of figuring out how to get staff more empathic in order to respond to YP's [young people's] need rather than simply react to their behaviour". 
Participant 12: "With regards to working on an emotional level - I practice being consistent with acknowledging them for who they are and working with the daily emotional turmoils. Some issues have arisen and we have said as a team we hear you, we understand your feelings and we are working through a process."

Utilising the skills. Almost all participants reported ways that they had used the skills taught on the training programme (13 participants, 48 diary entries). Most participants reported a range of skills they were using. For example, Participant 8 reported using at least five different techniques such as discussing talking points from the workbook with foster parents, teaching emotion coaching, and explaining how to manage difficult emotions. Participant 11 reported helping staff to be more empathic, putting difficulties into words for the child, focusing on relationships and:

Participant 11: “... emotionally connecting with the young person, responding to needs rather than reacting to the behaviour, iceberg notion, using some quotes of what to say to young people that I heard from other practitioners at the training”.

Almost all participants reported helping foster parents to work with the child's emotion, help the child name emotions, acknowledge feelings and give children more control. Two participants mentioned talking to foster parents about the child acting at a younger age for their years. Several participants who worked in residential care also talked about acknowledging young people more, ensuring they knew the worker "kept them in mind", bringing people in from the child's past and ensuring caregivers were not constantly changing. Participants also reported thinking more about who the young person turned to at times they needed support. One participant reported she used the skills with foster parents:

Participant 5: "by unpacking what may be going on for the child in their care and encouraging the caregiver to step back from the behaviour and use different strategies instead". 
Understanding the child. All participants reported either increased understanding of their own or of foster parents they were working with. One or two participants talked about how the compactness of the model helped parents to understand the child's behaviour. One participant said that a carer had realised the importance of meeting the child's need for birth family contact so that he didn't resort to acting out as he normally would.

Participant 16: “Carers looking at ways of meeting clients need to see his mother without compromising his wellbeing so the client does not need to behave in a manner that draws attention to himself or threaten self-harm."

The training also assisted the practitioners to think about their clients and their caregivers' point of view and some participants emphasised joint understanding. Practitioners described how they helped foster parents look at the causes of the child's behaviour to work out what might be driving it.

Participant 4: "[We] looked underneath behaviour and identified that child was anxious because of phone contact with the birth Mum."

Practitioners also mentioned that many caregivers focus on managing behaviour and emphasised the importance of understanding before action.

Participant 13: “This RFM [RLF] provides an improved understanding of this child's world view and why certain destructive behaviours are on-going. Having an understanding of this gives an understanding to then derive strategies to support this client's needs."

Participant 9: "It was great at creating a new perspective of the child for that foster parent. She was able to understand where the behaviour comes from (emotional needs) and how to respond."

A social worker talked about using the model before a child was placed permanently so that the foster parents could understand the child better. Other practitioners also reported looking at the child's perspective more and thinking about their behaviour more. 
Participant 8: "I encourage foster parents to recognise children's emotions and remind them where the children have come from and why they react and behave as they do. I use it to enhance the training I facilitate and have used the relational framework exercise to help foster parents have an understanding of a child before placing the child permanently."

Participant 3: "I was looking at things from my clients perspective."

Participant 2: “...and seeing/thinking/feeling things from a kid's point of view more.”

One practitioner commented that using the RLF can decrease the foster parent blaming the child when their behaviour management techniques are not working.

Participant 4: "This helps them to come into a more compassionate view of the child. With compassion, more possibilities open up to viewing the child's behaviour differently."

Participant 5: "Most of the children we work with have attachment issues and their behaviours reflect this in a myriad of ways, therefore in supervision I have used the light bulb training with social workers on how they can work with the caregivers to use an empathic approach with the child in their care, how to deal with the stress of caring for children not born to them, to think about how abuse and neglect can affect children's behaviour and how important it is for foster parents to try and separate behaviours form the child. I also encourage them to remind caregivers that children can act a lot younger than their actual age”.

Use of attachment theory. Practitioners reported viewing the clients' problems in a different way. Some participants had not been aware that there are different types of attachment and that these can be helpful to keep in mind when working with children. Most participants reported using attachment theory in their thinking about the child.

Participant 6: "I find I look at it through this Attachment framework lens now." 
Participant 2: "I was working and thinking with my 'attachment head' on."

Participant 13: "Constantly keeping in mind attachment while working with YP [young people]. Feeling more confident and educated in this area."

Several participants talked about the value of understanding attachment theory better and understanding the child's behaviour was related to attachment.

Participant 16: "Attachment issues have been to the forefront when working with a child/young person than it has been for a while."

Participant 14: “Focusing on bringing in positive people from past is important.

Regulating new people being introduced e.g. not having different respite carers all the time."

Participant 5: "I ...encourage them to consider the attachment issues of children who are in our custody... Most of the children we work with have attachment issues and their behaviours reflect this in a myriad of ways".

Some participants felt the training was a reminder of attachment theory rather than new material.

Participant 16: "This training has been helpful in reminding me about the different types of attachment and how to recognise them".

Participant 4: "Essentially there was nothing new but it was presented in such a way that I was able to make a number of new connections about how these processes work together - Lightbulb training is a very appropriate name."

Awareness and being mindful. Several practitioners reported that the training had made them more aware and mindful and that they found it insightful. They talked about doing more preparation, thinking more and reviewing plans more.

Participant 14: "The discussions are insightful and provide food for thought in all areas of child care and protection. The learning is ongoing and gives greater cause to improved understanding, knowledge and conversation." 
Participant 3: "I have probably been more mindful... I thought more about my approach to the YPs[young people], was more mindful of my words and how I was engaging with the YPs."

\section{Discussion}

Treatment satisfaction. The practitioner training programme was well received by practitioners with high attendance rates and high satisfaction ratings at post-test and at three month follow-up. Ratings at follow-up $(M=3.95)$ were very similar to post-test $(M=3.88)$ and were higher for the HSTEP Postcard $(\mathrm{M}=4.34)$, which rates use of knowledge and skills and observed client progress. Ratings indicated that participants found the group useful and would recommend it to others. Ratings also indicated that participants felt they had learned a substantial amount, had applied this learning in their work and saw themselves as a more effective worker as a result. Slightly lower, but still positive, ratings were given for changes in the participants' thinking, interaction with clients and the responses of clients to this change.

Practitioners' mind-mindedness. One aim of the study was to evaluate whether the Lightbulb training programme increases practitioner's mind-mindedness. Contrary to predictions, mind-mindedness scores did not significantly increase from pre- to post-test. Positive and negative mind-mindedness were also not significantly different. Mindmindedness has been used to evaluate only one training programme with foster parents and that study also found no difference between foster parent's mind-mindedness before and after the programme (Gurney-Smith et al., 2010). Mind-mindedness may not be a suitable measure for practitioners as a recent study showed that "mind-mindedness is a facet of personal relationships rather than a trait-like quality" (Meins, Fernyhough, \& Harris-Waller, 2014, p. 417). It may be that therapeutic relationships are not personal enough for this measure to be valid or that participants may simply not know enough about the client to fully answer the question. In this study, the mean mind-mindedness score for practitioners was slightly lower than that of the foster parents in study one and was also lower than the mean scores in two community samples (Lok \& McMahon, 2006; Meins et al., 2003).

Practitioners also had lower positive mind-related comments and higher negative mind-related comments when asked to describe the child, than those of foster parents in the first study. To the extent that mind-mindedness is a valid measure for use with practitioners, 
the positive or negative valence may mean different things for practitioners and parents. It would be expected that practitioners would use more negative mind-related comments as these would include common emotions a therapist might report such as anger and sadness. It is interesting then that practitioners did have a lower proportion of negative comments after the training programme, although this did not reach clinical significance.

\section{Practitioners' reflectiveness and empathy}

There was a significant increase in the proportion of empathic and reflective comments, with a medium effect size, made when participants discussed their thoughts about a scenario involving a foster child's difficult behaviour. This finding suggests that the training may have focused practitioners' attention on reflection and empathy. The training programme also significantly increased the total number of participant's empathic and reflective comments, with a small effect size. This may be the first time that an assessment using the "thinking aloud" technique has been used to show improvement in a key skill for social work and mental health practitioners.

Thematic analysis of practitioner interviews. A thematic analysis was conducted of diaries that the practitioners completed between training sessions and at follow-up answering three questions: a) What thoughts have you had about the training in your work from over the past month? b) Did you apply any of the learning to your work? c) What was the outcome? The questions were open so that practitioners had to think about their work and were not guided toward positive answers.

Practitioner's use of the training material. The thematic analysis showed that the majority of participants began using the theory and techniques from the training after the first training day. One participant used the RLF in its entirety and found this was too challenging to do on her own. Another participant noted that she was able to understand the different perspectives of her clients better and several participants commented that they were able to help the foster parent understand the child's behaviour. The aspects of the child's behaviour they mentioned were the underlying needs or drivers of behaviour. For example, one practitioner recognised that if the child's emotional need to see his mother was met in a safe way, then the child would not need to behave "in a manner that draws attention to himself or threaten self-harm". Another practitioner was able to reflect on the causes of the child's behaviour with the foster parent and came to the conclusion that anxiety about a phone call from the child's biological mother was fuelling the behaviour. 
These comments clearly show that the main aim of the training programme, to use the RLF to assist the practitioner to understand the child's behaviour and assist the foster parent to do so, was achieved. Participants were clearly oriented toward thinking about the child's behaviour, in terms of the child's past and present experiences. This is crucial for foster children whose behaviour may be misunderstood, as the roots in trauma and maltreatment may be either unknown or not focused on (Lawler, 2008; Tarren-Sweeney, 2008). This also suggests that practitioners can assist foster parents who make negative attributions about the child's behaviour, such as the child is being naughty or the foster parent lacks skills, rather than understanding the genesis of the behaviour in the child's learning from adverse experiences.

Practitioners discussed better understanding the effects of abuse and neglect after doing the RLF, as the framework clarifies what aspects of the child's functioning may have been affected. They further reported that using the framework helped foster parents have a more compassionate view of the child when they were dealing with difficult behaviour. A practitioner reported that a foster parent had thought the child was engaging in certain behaviours to annoy her and when the foster parent had more understanding of the behaviour, she was able to be more compassionate, and this was accompanied by an improvement in the child's behaviour. Practitioners said they used training material with foster parents whose children were in therapy with them and for preparation for foster parents taking permanency for a child. They also used it to organise their thoughts before writing a report and when preparing for a young person to come into their service. A participant found the training material a "helpful and accessible way" to give the young person information.

The training clearly focussed practitioners' thinking on attachment theory and the importance of considering the child's emotions as well as behaviour. Some practitioners expressed frustration that residential staff would respond with behavioural techniques to the young person's behaviour when it was clearly expressing an emotional need.

The practitioners talked about adding the attachment theory framework to behavioural methods, rather than replacing them. They also reported using the information to advocate for the child, presenting the case for what the child needed and supporting the young person to advocate for themselves. They encouraged emotional connection and taught foster parents and residential staff emotional validation techniques. Participants reported they changed their theoretical conceptualisation of the child. Many practitioners talked about considering attachment theory or thinking about attachment more, which indicates increased attention to the relational aspects of the child's situation. Practitioners also appreciated the small group 
format of the training and several participants reported using other participants' ideas, which had been discussed in the group.

Comments on the use of the training material were virtually all positive, with participants not reporting any difficulties presenting techniques to clients or any negative reactions, either of the client or their own. This suggests the training material can be implemented successfully and resulted in positive outcomes, at least as reported by practitioners. Negative experiences were confined to not having sufficient time to do the RLF and the time to work through issues with foster parents or residential social workers when consulting. Some practitioners mentioned that changing the solely behavioural focus of some carers was challenging.

Training material practitioners did not use. The RLF method suggests that practitioners help foster parents to put ideas about the causes of the child's behaviour into words for the child in a simple way, for example, "no wonder you are shouting today, that was a difficult visit from your Dad". The idea behind this suggestion is that parents who reflect and talk to a child about their behaviour, help the child to reflect about themselves and other people (Schofield \& Beek, 2005a). Practitioner diaries revealed that practitioners seldom reported using this technique. They gave various examples of helping foster parents understand the child and commented on the positive difference this made but they did not mention helping the foster parents to put this into words for the child. It is possible that this was too hard for practitioners to do or it may be that they did not see this as useful for the foster parent.

One aspect of the child's functioning the participants were taught was that children in foster care who have been abandoned by, or removed from, their own biological parent, sometimes fear that they will be forgotten by new caregivers. They are also taught to let the child know they think about the child, or keep the child in mind, when they are apart. This can be as simple as "I thought about you sitting your test after lunch today." Some practitioners did report using this technique. While practitioners did not often mention this in their diaries, a residential social worker commented that she acknowledged the young person more and told the young person that she had been thinking about her. Unfortunately, the diary indicated that the practitioner may have needed more direction to make these comments in a detailed and meaningful way, that is, to apply them to concrete activities in the child's life, to indicate that the child is genuinely remembered.

Summary. The results of the thematic analysis show that foster care practitioners learned and retained novel theoretical perspectives and techniques in the training programme. They applied these to their work in a variety of ways, with both foster parents and foster 
children, and they reported positive outcomes. They thought about clients in new ways, especially the application of attachment theory, and found they had gained insight into the child's perspective. They applied emotion validation techniques productively. Two agencies have adopted the RLF as part of their intake procedures and participants reported continuing to use these methods with positive results at three month follow-up. One subsequent training group for foster parents has been completed and several individuals have worked through the programme with a therapist or psychologist.

The advantage of using qualitative methods is that they encourage participants to think about their experience. A qualitative diary method allows an appraisal of whether the practitioners actually used the material. The diaries themselves may have focused practitioners on using the training material, although many practitioners filled the diaries in just before the next group and did not appear to spend much time reflecting on the questions. Spacing the training days either fortnightly or monthly, and having multiple training days, may have assisted practitioners to apply the material in between times. It also meant that they could ask questions about the material and gain proficiency by receiving feedback, and also hearing what other practitioners had done.

\section{Strengths and Limitations}

The main limitation of this study is its pre- post-test design. A multiple-baseline design could not be utilised due to the lack of an appropriate time-series measure, since the practitioners worked with the child once a week, or during the working week in the case of residential social workers, but did not live with the child. In order to make up for this deficit, qualitative information was gathered to assess what practitioners actually thought about and applied in between the training sessions. The assessment of mental representations and cognitions may be a first in professional training, and yet this provides a realistic measure of the impact on the participants' thinking, which is after all a main aim of worker training, alongside behaviour change.

In addition, the sample was small and mainly from two agencies. Half of the participants worked with foster children with sexually harmful behaviour so this may limit generalisation to other foster children but the agency also worked with foster children without sexually harmful behaviour. 


\section{Conclusions}

This study may be the first to use assessments of mental representations and cognitions in evaluating a professional training programme. The programme was effective in increasing practitioners' empathic and reflective comments but mind-mindedness scores were not significantly different. Mind-mindedness may not be a suitable measure for professionals who are not in a parental role. A thematic analysis of practitioner diaries showed that the Lightbulb training group was well received by practitioners who began utilising the techniques and principles taught immediately after the first training day. They reported the techniques had positive outcomes for work with clients and that they were more aware of clients' perspectives. They utilised theoretical concepts presented in the training course and reported thinking more about their clients. 


\section{Chapter Nine-Overall discussion and conclusions}

Foster parents may have little understanding of the way children in their care have come to view relationships, as the foster parent is usually not present in the child's early life. This may make it difficult for foster parents to accurately interpret children's behavioural signals and successfully manage their behaviour problems. The theoretical proposition underlying this thesis is that children construct an internal working model of interpersonal relationships, based on the way they are cared for by their parents. As many foster children have an array of difficult behaviours, the question arose as to whether foster parents understanding of this internal working model would be helpful. If foster parents understand the child's behavioural signals, which are related to their internal working model, they may respond more positively, and further may be able to assist the child to understand and modify their behaviours.

The first aim of the thesis, then, was to investigate whether foster parents understanding of the child's perspective is positively associated with the child's functioning. A broad view was taken, influenced by ecological transactional theory, which emphasises the reciprocal relationships among factors affecting child development. This includes the wider context of children's previous experiences and the psychological functioning of the foster parent. The factors examined in the research were aspects of parent psychological functioning found to be associated with children's socio-emotional development. Key amongst these variables are mind-mindedness, attachment perceptions and emotional availability. This is the first study to explore these aspects of foster parents' psychological functioning together, while taking an array of foster child characteristics into account. The second aim of the thesis was to develop, and subsequently evaluate, a training programme designed to help foster parents and foster care practitioners understand the child's internal working model and, in turn, to enhance their understanding and management of the child's behaviour.

\section{Key findings: parent factors}

Overall, the results showed a complex interaction between foster child and foster parent factors. Foster parents who were more mind-minded had children with fewer behaviour problems. Additionally, foster-parents were found to be more mind-minded with younger children, and children living with them longer term. This suggests that the association of mind-mindedness is bidirectional - the child's characteristics are associated with the parents' mind-mindedness and the parents' mind-mindedness is associated with the child's emotional 
and behaviour problems. This finding is important, as previous studies have found that the maintenance and severity of foster children's emotional and behaviour problems are most likely to be related to their prior history of adverse experiences (Berzin, 2008; Biehal, Ellison, Baker et al., 2011). This research suggests individual differences in foster parents' tendency to consider the child's mental and emotional life may also play a part.

A major finding concerned the interaction of different components of foster parent's psychological functioning. When parents were more mind-minded their child had fewer behaviour problems but the quality of the parent child relationship was unrelated. When foster parents demonstrated more mind-mindedness, and positive attachment perceptions, mind-mindedness was more strongly associated with the child's emotional and behaviour problems and became associated with the parent-child relationship. This finding suggests that these two aspects of foster parent's own functioning work together to predict the child's outcomes.

There are at least two possible explanations for the interaction between foster parent attachment perceptions and mind-mindedness. First, parents who are more able to explain their own and other people's behaviour when discussing personal relationships, by talking about mental states, are also more likely to focus on their children's mental states (Bernier \& Dozier, 2003; Meins et al., 2001). Second, self-reported attachment focuses on the adult's negative emotions about their primary relationship in childhood and high levels of negative emotions may affect the parent's clarity or willingness to see the child's perspective.

A further key finding was that foster parents' attachment perceptions were a more robust predictor of both outcome variables than foster parent mind-mindedness. Bernier and Dozier (2003) have argued that mind-mindedness would be expected to be a more robust predictor of children's attachment security as mind-mindedness is " child specific and therefore closer to the actual relationship with the child than a parent's account of his or her own childhood experiences" (p. 36). The current findings do not support this hypothesis and are consistent with attachment research, which has found that parent's state of mind about attachment is the most robust predictor of children's attachment security (van IJzendoorn, 1995).

\section{Key findings: child factors}

Younger children, children placed at a younger age and children with fewer placement changes were reported to have better mental health and better relationships with their foster 
parents. These findings are well-established in the literature, although the details may conflict in different studies (Nutt, 2006; Oosterman et al., 2007; Tarren-Sweeney, 2008a). These finding suggest that the current sample is similar to others and findings can, therefore, be generalised.

Children's socio-emotional functioning was related to two groups of variables, which cohere together. The first is that the younger the child is, and the younger age they are placed, the better the foster parent relationship is with the child. These findings may reflect a number of factors - younger children are still within or are closer to the age of normative development of attachment (Sroufe, 2000;Thompson, 2000) and younger children may be more adaptable and have fewer entrenched problems. The second is that factors reflecting placement instability (more previous placements, short term placements) were associated with both the child's emotional and behaviour problems and the quality of the parent-child relationship. This finding may be explained by a number of considerations - the child's loss of trust, grief, and fear for the future (Unrau et al., 2008, Unrau et al., 2011) and the severing of social connections (Ryan \& Testa, 2005). Foster parents may have incrementally less influence over the child's behaviour problems, when bonds with biological parents and previous foster parents have been severed more often.

The relationship between foster parents' attachment perceptions and children's outcomes was also altered by the child's characteristics. Foster parents' attachment perceptions were related to younger children's behaviour problems and the foster parent-child relationship with boys. In the small sample of foster parents and children who completed a video rated for emotional availability parents were observed to respond differently to children with different characteristics. For example, foster parents were rated as more sensitive and less intrusive to children placed at a younger age. These findings provide further evidence that both foster parent and child variables work in conjunction to predict child outcomes.

\section{Key findings: Intervention}

Foster parent intervention. The main aim of this pilot intervention study was to examine whether a training programme designed to enhance foster parents understanding of the foster child was effective in improving the participants focus on the child's mind and emotions (mind-mindedness). Findings were mixed. Foster parents evaluated the Lightbulb training programme positively. The intervention showed a significant increase in foster parent positive mind-mindedness at follow up, and modified Brinley plots showed shifts in 
the direction of improvement in both positive and negative mind-mindedness at follow-up. Significant improvements were also found at post-test in several measures of foster children's behaviour problems, attachment relationships and overall socio-emotional functioning. By follow-up, however, only the child's overall socio-emotional functioning and positive mindmindedness were significantly improved, and there was a decrease in foster parent wellbeing. Although the effectiveness of brief psycho-education programmes has generally been poor, this study showed significant improvements in children's daily behaviour and overall behaviour problems, in the short term. This suggests a replication would be worthwhile as long as two modifications to the programme were carried out: the foster parent stress module requires strengthening and the maintenance of effects needs attention. One option is to include the foster parent's social worker in part of the programme or to offer support sessions after the training is completed.

In conclusion, although limited by a small sample and improvements in the measures over the course of the baseline, there were short-term improvements in foster child functioning and aspects of foster parent mind-mindedness after the Lightbulb training programme, which are encouraging in the context of a short term intervention with this high risk group. The individual focus of the RLF, in contrast to teaching general principles and skills, may be a useful addition to a group format. This is the first study to show even limited evidence that aspects of foster parent mind-mindedness can be enhanced by a training programme. The programme aimed to improve the parents' mind-mindedness in an indirect way, that is, to better understand the child's perspective, by learning about the relational impacts of past adverse experiences. It is possible that results could be improved by teaching mind-mindedness directly, that is, how to name what the child is thinking and feeling (Colonessi et al., 2012).

Foster care practitioner intervention. Foster care practitioners also evaluated the Lightbulb training programme positively. The intervention significantly increased foster care practitioners' empathic and reflective comments, when asked to report their thoughts about a foster care scenario. This is an important finding as few studies evaluating training programmes for practitioners have measured participants' cognitions (Zanov \& Davison, 2010). There were no significant improvements in practitioner mind-mindedness after the intervention. Mind-mindedness may not be a suitable measure for those in a professional role with the child, as they may not know the child well enough for the measure to be valid (Meins et al., 2014). 
Diary methods allow an appraisal of whether the practitioners actually used the material. This is important as training will not be used if people do not have time to process it and think about how to apply it (Antle et al., 2008). Practitioner diaries clearly showed that participants applied a variety of the techniques they had learned in the training in their work. Practitioners reported that they had a greater understanding of the foster child's behaviour. When they helped the foster parent to reflect on the child's behaviour, outcomes were positive. They also reported that foster parents were able to emotionally validate and connect with the young person after applying the techniques. Participants also reported using "an attachment lens" more often, indicating a shift in theoretical stance. Few practitioners reported difficulties in implementing the strategies.

\section{Use of a multiple-baseline research design}

This research showed that a multiple-baseline study has much to recommend it in a pilot study testing an intervention. First, it allows tentative conclusions that any therapeutic effect is due to the intervention rather than other factors. Second, using modified Brinley plots allows a visual examination of any participant with deteriorated scores. These figures are hidden in group analyses and yet they are important as they may suggest the programme requires modification, as is likely in the current training programme. Modified Brinley plots also show the strengths and weaknesses of the programme, by contrasting shifts towards and away from improvement, which again aids in modification of the programme. A third benefit of modified Brinley plots is that the band of measurement error can be clearly plotted so the size of any effects is clearly noticeable.

In conclusion, the use of the Lightbulb training programme, modified for foster care practitioners, was rated highly by the participants, who showed increased empathic and reflective comments when reporting their thoughts about a foster child and that the programme made them more effective in their work. These results suggest that the Lightbulb training programme can enhance the clinical and social work practice of practitioners, in this complex area of work. It has the potential to strengthen foster placements through enhancing foster parent understanding of the child's perspective.

\section{Implications for practice and future directions}

In this research, mind-minded parents had children with fewer emotional and behaviour problems. While causality cannot be determined by a cross-sectional study, these findings could be extended to examine whether foster parents mind-mindedness is associated with the 
child's emotional and behavioural problems, as reported by more than one informant, or whether mind-minded foster parents view children's behaviour with more understanding, and thus report fewer problems. A further extension requires the use of a longitudinal study with a sufficiently large sample to investigate relationships between factors over time. Such a study could include foster children themselves and could use the Relatedness Questionnaire (Lynch \& Cicchetti, 1997). This questionnaire includes questions about the child's relationships with their biological parents, foster parents, and teachers. It would be valuable to understand the associations between the child's perception of their relationships and the child's outcomes.

The current body of work suggests that there is great potential in harnessing and matching aspects of the foster parents' psychological functioning and the child's history, in order to improve the currently poor outcomes for foster children. Foster parent mindmindedness and attachment perceptions are potential targets to improve the selection of foster parents, particularly for older children or young people with more severe behaviour problems who could be matched with foster parents with high mind-mindedness and positive attachment perceptions. These factors can also be incorporated into foster parent training, which could be improved in three ways: a) by directly teaching foster parents to name the child's thoughts and feelings b) by increasing the foster parents' understanding of the child's perspective and c) by reflecting on the impact of the foster parent's attachment perceptions on their relationship with the child.

Important related research questions include why young foster children become securely attached at similar rates to biological children, and yet mental health problems may persist into adulthood. A further question is how children's internal working models change after they have been exposed to maltreatment and removal from home. Little is known about how children update their internal working model when care improves and this is a crucial area for foster care research, with implications for intervention for both foster parents and children.

\section{Conclusions}

The studies in this thesis extend the literature on the effect of parents' mental representations on children's functioning, by showing that the way foster parents think about the foster child's mental life and emotions is associated with the child's emotional and behaviour problems and the foster parent-child relationship. This association is over and 
above some of the prior negative experiences of the foster child, such as frequent placement changes. Moreover, this research extends findings on the importance of the foster parent's state of mind about attachment, by showing that parents self-reported attachment perceptions enhance the effect of mind-mindedness on both the child's emotional and behaviour problems and the quality of the parent-child relationship. In addition, the research shows that parent psychological functioning is also related to the child's characteristics such as the child's age, the age at which the child was placed and previous placement changes, indicating a bidirectional relationship. 


\section{Appendix A-Group Study Interview (semi-structured)}

Part 1: I will be asking you a number of questions about yourself, your family and your foster child. If you don't want to answer any questions just say so and if you wish to stop the interview at any point, please let me know. Some of the questions are personal but are included as they may affect how you are caring for the child in your care.

\begin{tabular}{|c|c|c|}
\hline Gender: Self & $\mathrm{F}$ & $\mathrm{M}$ \\
\hline Gender Child (first name) & $\mathrm{F}$ & $\mathrm{M}$ \\
\hline \multicolumn{3}{|l|}{ Age: Self } \\
\hline \multicolumn{3}{|l|}{ Age: Child } \\
\hline \multicolumn{3}{|l|}{$\begin{array}{l}\text { Highest educational qualification (or last } \\
\text { year at school) }\end{array}$} \\
\hline \multicolumn{3}{|l|}{ Ethnicity: Self } \\
\hline \multicolumn{3}{|l|}{ Ethnicity: Child } \\
\hline \multicolumn{3}{|l|}{ Is the child related to you? } \\
\hline \multicolumn{3}{|l|}{ Do you have a partner? } \\
\hline \multicolumn{3}{|l|}{$\begin{array}{l}\text { How many children do you have at } \\
\text { home? [birth, foster] All at home? }\end{array}$} \\
\hline \multicolumn{3}{|l|}{ How many children have you fostered? } \\
\hline \multicolumn{3}{|l|}{$\begin{array}{l}\text { Any left because behaviour couldn't be } \\
\text { managed? }\end{array}$} \\
\hline \multicolumn{3}{|l|}{$\begin{array}{l}\text { Have you done any training about } \\
\text { providing foster care? (hours/days) }\end{array}$} \\
\hline \multicolumn{3}{|l|}{$\begin{array}{l}\text { How long has the child been in your } \\
\text { care? }\end{array}$} \\
\hline \multicolumn{3}{|l|}{$\begin{array}{l}\text { How many placements had the child had } \\
\text { before coming into your care? (List all } \\
\text { changes of caregiver) }\end{array}$} \\
\hline \multicolumn{3}{|l|}{ Why did the child come into care? } \\
\hline $\begin{array}{l}\text { Has the child experienced any type of } \\
\text { abuse - physical, sexual or emotional? }\end{array}$ & & \\
\hline
\end{tabular}




\begin{tabular}{|l|l|}
\hline $\begin{array}{l}\text { Is the child still in contact with any } \\
\text { members of his or her birth family - } \\
\text { mother, father, grandparents, aunts and } \\
\text { uncles, other. }\end{array}$ & \\
\hline $\begin{array}{l}\text { Does the child have any respite care? } \\
\text { (How much, how often?) }\end{array}$ & \\
\hline $\begin{array}{l}\text { Does the child have any medical, mental } \\
\text { health or disability problems? }\end{array}$ & \\
\hline $\begin{array}{l}\text { Do you have concerns about the child's } \\
\text { behaviour? }\end{array}$ & \\
\hline $\begin{array}{l}\text { Does the child get any extra help such as } \\
\text { attending therapy, teacher aide? }\end{array}$ & \\
\hline $\begin{array}{l}\text { Do you have a social worker who visits } \\
\text { you? }\end{array}$ & \\
\hline $\begin{array}{l}\text { Does having a foster child cause you any } \\
\text { stress? If so what? (behaviour, } \\
\text { emotional, financial?) }\end{array}$ & \\
\hline $\begin{array}{l}\text { A few questions about your own } \\
\text { experiences - have you yourself } \\
\text { were growing up? }\end{array}$ & \\
\hline sid you experience any neglect, or abuse & \\
\hline
\end{tabular}

\section{Part 2: Mind-mindedness Interview}

Now, I have a number of questions about your foster child and how you feel about looking after him or her. I would like to begin by asking you to describe (child). [Probe question: What is he/she like?]

\section{Part 3: "This is my Baby [Child]" Interview}

1. Do you ever wish you could raise (child's name)?

2. How much would you miss (child's name) if he/she had to leave? 
3. How do you think your relationship with (child's name) is affecting him/her right now?

4. How do you think your relationship with (child's name) will affect him/her in the long term?

5. What do you want for (child's name) right now?

6. What do you want for (child's name) in the future?

7. Is there anything about (child's name) or your relationship that we've not touched on that you'd like to tell me?

\section{Part 4: Child behaviour and attributions}

I'd like you to think about the last time [name] showed difficult behaviour or got into trouble

1. What did he/she do?

2. Why did he/she do that i.e. what were the main reasons for the behaviour?

3. How was he/she feeling?

4. What did you say and/or do?

5. How did [name] respond?

6. Did you talk about it later? If, so, what did you say?

In your opinion, to how much did this behaviour have anything to do with:

\begin{tabular}{|c|c|c|c|c|c|c|}
\hline The child's personality? & 1 & 2 & 3 & 4 & 5 & 6 \\
\hline The situation at the time? & 1 & 2 & 3 & 4 & 5 & 6 \\
\hline The child's past experiences? & 1 & 2 & 3 & 4 & 5 & 6 \\
\hline The child's relationship with you? & 1 & 2 & 3 & 4 & 5 & 6 \\
\hline The child being in foster care? & 1 & 2 & 3 & 4 & $J$ & 6 \\
\hline $\begin{array}{l}\text { The child having been in } \\
\text { several placements? }\end{array}$ & 1 & 2 & 3 & 4 & 5 & 6 \\
\hline Your parenting of the child? & 1 & 2 & 3 & 4 & 5 & 6 \\
\hline The child's friends? & 1 & 2 & 3 & 4 & 5 & 6 \\
\hline The influence of the child's & 1 & 2 & 3 & 4 & 5 & 6 \\
\hline
\end{tabular}

birth parents? 


\section{Appendix B-Correlations for the Emotional Availability Scale (4 ${ }^{\text {th }}$ ed.)}

\begin{tabular}{|c|c|c|c|c|c|c|c|c|c|c|c|c|c|c|c|c|}
\hline & 1. & 2. & 3. & 4. & 5. & 6. & 7. & 8. & 9. & 10. & 11. & 12. & 13. & 14. & 15. & 16. \\
\hline 1.Age & & & & & & & & & & & & & & & & \\
\hline 2. Placement length & $.29^{*}$ & & & & & & & & & & & & & & & \\
\hline 3. Age placed & $.67 * * *$ & $-.54 * * *$ & & & & & & & & & & & & & & \\
\hline 4. Number of placements & $.27 *$ & $-.27 *$ & $.45 * * *$ & & & & & & & & & & & & & \\
\hline 5. Abuse & .12 & -.10 & .22 & $.23 *$ & & & & & & & & & & & & \\
\hline 6. Short term placement & -.07 & $.46^{* * * *}$ & $.33 * *$ & .22 & $-.31 *$ & & & & & & & & & & & \\
\hline 7. MM & $-.28 *$ & -.07 & -.13 & ,04 & -.18 & $.26^{*}$ & & & & & & & & & & \\
\hline 8. Positive MM & $-.25^{*}$ & .001 & -.24 & -.19 & $-.32 *$ & $.37 * *$ & .11 & & & & & & & & & \\
\hline 9. Negative MM & .21 & .12 & .08 & .09 & $.34 *$ & -.20 & -.02 & $-.72 * * *$ & & & & & & & & \\
\hline 10. EA Sensitivity & -.44 & .20 & $-.50 *$ & -.22 & -.43 & -.03 & -.06 & .10 & -.08 & & & & & & & \\
\hline 11. EA Structuring & -.31 & .33 & -.30 & -.19 & -.06 & -.04 & .18 & -.18 & .20 & $.67 * *$ & & & & & & \\
\hline 12. EA non-intrusiveness & -.22 & $.51 *$ & $-.45^{*}$ & $-.47 *$ & -.23 & .25 & .04 & -.06 & .15 & $.69^{* *}$ & $.51 *$ & & & & & \\
\hline 13. EA Non-hostility & -.15 & .21 & -.38 & -.22 & $-.60 * *$ & $\begin{array}{l}.09 \\
\end{array}$ & .02 & .02 & .02 & $.75 * * *$ & $.63^{* *}$ & $.50 *$ & & & & \\
\hline 14. EA Child Responsive & -.20 & $.44 *$ & $-.67 * *$ & -.18 & -.18 & .40 & .10 & .06 & -.17 & $.71 * * *$ & $.61^{* *}$ & $.57 * *$ & $.63^{* *}$ & & & \\
\hline 15. EA Child Involve & -.36 & .26 & $-.58 * *$ & -.16 & -.42 & .09 & .08 & -.03 & -.01 & $.79 * * *$ & $.69^{* *}$ & .41 & $.65^{* *}$ & $.91 * * *$ & & \\
\hline 16. SDQ & .13 & -.19 & .24 & $.28 *$ & $.35^{* *}$ & $.35 * *$ & $-.31 *$ & $-.37 * *$ & $.40^{* *}$ & .00 & .11 & .12 & -.05 & -.24 & -.27 & \\
\hline 17. CPRS & $-.33^{*}$ & .15 & $.41 * *$ & $-.29 *$ & -.17 & $.31 *$ & .19 & $.32 *$ & $-.37 * *$ & .24 & .00 & .00 & .14 & .43 & $.52 *$ & $-71 * * *$ \\
\hline
\end{tabular}

Note. ${ }^{*} p<.05 ; * * p<.01$, two tailed significance. Due to one participants not completing questionnaires, $N=21$ for variables $1-15 ; N=20$ for variables $16-$

17. $. \mathrm{SDQ}=$ Strengths and Difficulties Questionnaire; CPRS $=$ Child-Parent Relationship Scale; MM = Mind-mindedness; PAAQ = Perceptions of Adult Attachment Questionnaire. 


\section{Appendix C-Articulated thoughts in simulated situations (ATSS) procedure}

\section{Instructions for delivering ATSS protocol}

Please give the following instructions before each scenario.

"I am going to read you two scenarios involving a foster parent and child. Please imagine you are in the situation and when asked, please say what is running through your mind. There are no right or wrong answers and we simply want your thoughts about what you hear. We will then ask you how you would deal with a similar situation".

If parents are unsure please indicate that:

There are no right or wrong answers. We are interested in what the parent thinks and would do. The reaction question relates to their own foster child.

In part 2 of the scenario, if the parent says they would talk to the social worker, ask them what they would do or say in the meantime if the social worker wasn't available. 


\section{Scenario 1}

You have been fostering a 10 year old girl for the past year. On this particular day, she rushes in from school and says that her teacher is an "ugly bitch" for giving her a detention. You ask her what she did to get the detention and she screams at you "You always take her side and you never listen to me". She storms off into her bedroom.

Please say aloud everything that is running through your head about what you have just heard

You go after her and she bursts into tears and tells you that her teacher gave her the detention for stealing a girl's lunch but that she didn't do it and her friend can back her up on that. She cries and says everyone picks on her, she's got hardly any friends and they say she's a welfare kid.

Please say aloud everything that is running through your head about what you have just heard

You talk about it a bit and then get on with the day. Later on you are looking for something in her bag and you find a half eaten lunch that is not her's. You also find she has scribbled words like HATE and GANGZ RULE all over her homework book.

Please say aloud everything that is running through your head about what you have just heard

If you had to deal with your child crying because she was picked on what kinds of things would you do? Which of those are you most likely to carry out? How would your child react?

If you had to deal with your child lying to you what kinds of things would you do? Which of those are you most likely to carry out? How would your child react? 


\section{Scenario 2}

You have been fostering an 8 year old girl for 2 years. She is usually a happy child with a bubbly personality. You have just discovered that she has been hiding dirty clothes under the bed and there is now a pile of smelly, rotting clothes which have stained the carpet as some of them were soiled i.e. she hadn't made it to the toilet on time.

Please say aloud everything that is running through your head about what you have just heard

When you talk to her about it she is very sad and says she was really scared that you would tell her off and punish her. She said her birth Mum would strap her when she did wees or poos in her pants and she thought you might do it too.

Please say aloud everything that is running through your head about what you have just heard

You assure her that you will not hit her no matter what and that you are not angry with her now that you understand why she did it. She feels better about this but follows you around the house for the rest of the day, cries at the slightest problem and clings to you when it's bedtime.

Please say aloud everything that is running through your head about what you have just heard

If you had to deal with your child hiding smelly clothes under her bed what kinds of things would you do? Which of those are you most likely to carry out? How would your child react?

If you had to deal with your child not letting you out of her sight and clinging to you what kinds of things would you do? Which of those are you most likely to carry out? How would your child react? 


\section{Scenario 3}

You have been fostering a 9 year old boy for 6 months. You are sorting out household chores for the year. You tell him that he has to do two chores and ask him to choose between several easy tasks. He tells you that you are a slave driver and if you make him do chores he will tell Child, Youth and family and he will run away if they say he has to do them.

Please say aloud everything that is running through your head about what you have just heard

You tell Sam that everyone does chores and that children he knows at school do them. He says that's not right and you are only making him do them because he is a foster child and you don't care about him.

Please say aloud everything that is running through your head about what you have just heard

He says you are only doing foster care for the money. He says he doesn't like you and he's been thinking of running away for a while. He says you are mean for making him do things and that his mother never used to make him do anything.

Please say aloud everything that is running through your head about what you have just heard

If you had to deal with your foster child telling you that you are not a good parent what kinds of things would you do or say? Which of those are you most likely to carry out? How would your child react?

If you had to deal with your child threatening to tell Child, Youth and Family about you what kinds of things would you do or say? Which of those are you most likely to carry out? How would your child react? 


\section{Scenario 4}

You have been fostering a 12 year old boy for 18 months. You have just learned that he has stolen your sister's ipod and sold it at school for $\$ 10$. There is no way of getting it back. Your sister had thousands of songs on her ipod and is very upset. She wants to confront him and she thinks you should send him to another foster home.

Please say aloud everything that is running through your head about what you have just heard

You talk to him about it and he starts begging you not to tell Child, Youth and Family. He says he'll do anything to make it up to you and your sister. He doesn't deny selling the ipod, he just wants you to keep quiet about it. He starts watching television and refuses to talk about it anymore.

Please say aloud everything that is running through your head about what you have just heard

You tell him that you can't ignore it and that you can't keep secrets from his social worker. He starts crying and saying you will ruin his life and that nobody wants him. $\mathrm{He}$ says that everyone sends him away when he does bad things and you're just like them.

Please say aloud everything that is running through your head about what you have just heard

If you had to deal with your foster child refusing to speak to you about something naughty they had done what kinds of things would you do or say? Which of those are you most likely to carry out? How would your child react?

If you had to deal with your foster child starting to pack their bags after threatening to run away what kinds of things would you do or say? Which of those are you most likely to carry out? How would your child react? 


\section{Scenario 5}

You have been fostering a 6 year old boy for 3 months. He is very scared of the dark and is convinced that burglars will come and take him away. Every night he asks you are all the doors locked and where his Dad is. His Dad is serving time in jail for violence against Max and his mother.

Please say aloud everything that is running through your head about what you have just heard

You tell him his Dad cannot hurt him anymore because he is in jail and Max cries and says he misses his Dad and wants to visit him. He also misses his Mum who has moved away and who doesn't have any contact with Max.

Please say aloud everything that is running through your head about what you have just heard

Max says he loves his Dad and that he only hit him when he was drunk. He says he wants to see his Dad because he is fun but he also wants to make sure he really is locked up. He cries and asks you will you be his new parent and will you save him if his Dad tries to take him away?

Please say aloud everything that is running through your head about what you have just heard

If you had to deal with your foster child feeling frightened he was going to be kidnapped what kinds of things would you do or say? Which of those are you most likely to carry out? How would your child react?

If you had to deal with your foster child asking you to be his new parent what kinds of things would you do or say? Which of those are you most likely to carry out? How would your child react? 


\section{Scenario 6}

You have had a 12 year old foster child living with your family for the past year. She has recently found out that her Dad and his new partner have a new baby. She is desperate to see the baby and feels that you are stopping her from seeing the baby and also keeping her away from her Dad. She is becoming very withdrawn and spends a lot of time on her own in her room.

Please say aloud everything that is running through your head about what you have just heard

You explain to her that social workers make decisions like that and she says it doesn't matter anyway because no one listens to her and she'll never get to live with her Dad. She says in a snarky voice "What would you know anyway, you've never been a foster kid!".

Please say aloud everything that is running through your head about what you have just heard

Your foster child is ok for a few days after this talk and then the school rings you to say she has wagged two classes and been caught smoking today. Isabel arrives home and looks worried about what you are going to say.

Please say aloud everything that is running through your head about what you have just heard

If you had to deal with your foster child blaming you for not being able to live with his or her parents, what kinds of things would you do or say? Which of those are you most likely to carry out? How would your child react?

If you had to deal with your foster child starting to wag school and smoke cigarettes what kinds of things would you do or say? Which of those are you most likely to carry out? How would your child react? 


\section{Appendix D—ATSS coding}

Please code all instances of the following responses to a difficult situation with a foster child

\begin{tabular}{|c|c|c|}
\hline Code & Definition & Examples \\
\hline Empathy (E) & $\begin{array}{l}\text { Empathy, validation and } \\
\text { acknowledgment of the } \\
\text { child's or foster parent's } \\
\text { feelings or mental states. } \\
\text { E.g. feeling neglected. } \\
\text { Include getting the } \\
\text { child's perspective. Any } \\
\text { statement about feelings }\end{array}$ & $\begin{array}{l}\text { I would talk to the child about their feelings. } \\
\text { I would say something like "You must feel } \\
\text { sad about that" } \\
\text { Talking to the foster child about his or her } \\
\text { feelings or mental states such as boredom, } \\
\text { excitement. } \\
\text { Talking to the foster parent about their own } \\
\text { or their child's feelings or mental states such } \\
\text { as boredom, excitement. } \\
\text { How did you feel about that? } \\
\text { I would validate her feelings. } \\
\text { I would empathise. } \\
\text { She was sad. }\end{array}$ \\
\hline $\begin{array}{l}\text { Reflection - } \\
\text { behaviour/general } \\
\text { (RB) }\end{array}$ & $\begin{array}{l}\text { Reflecting about the } \\
\text { underlying causes of the } \\
\text { child's behaviour which } \\
\text { are in the present } \\
\text { environment of home or } \\
\text { school or are very } \\
\text { general . Also for } \\
\text { comments about current } \\
\text { interactions with the } \\
\text { birth family e.g. access. } \\
\text { Triggers. Why did the } \\
\text { child behave that way } \\
\text { and why now? }\end{array}$ & $\begin{array}{l}\text { I thought about why she did that. } \\
\text { I decided to help her figure out why she did } \\
\text { that. } \\
\text { I explained what happens when you feel... } \\
\text { Of course she's clingy because our other son } \\
\text { is in hospital. } \\
\text { She's struggling with school so she's very } \\
\text { moody when she gets home. } \\
\text { All children do that when ... }\end{array}$ \\
\hline $\begin{array}{l}\text { Reflection - } \\
\text { foster care and }\end{array}$ & $\begin{array}{l}\text { Making connections to } \\
\text { the child's past - }\end{array}$ & $\begin{array}{l}\text { Maybe it's because she was brought up in a } \\
\text { gang. }\end{array}$ \\
\hline
\end{tabular}




\begin{tabular}{|c|c|c|}
\hline $\begin{array}{l}\text { maltreatment } \\
(\mathrm{RFC})\end{array}$ & $\begin{array}{l}\text { thinking about the } \\
\text { position of the foster } \\
\text { child, how they might } \\
\text { feel being away from } \\
\text { their family and how } \\
\text { they might fear being } \\
\text { sent away again. Also } \\
\text { the effects of } \\
\text { maltreatment. This } \\
\text { includes both: } \\
\text { 1) Foster parents or } \\
\text { practitioner's } \\
\text { general } \\
\text { knowledge of } \\
\text { foster children } \\
\text { 2) This child's } \\
\text { experience }\end{array}$ & $\begin{array}{l}\text { Foster children may feel... } \\
\text { She lived with drug addicts so she saw a lot } \\
\text { of violence when they didn't pay up. } \\
\text { The kids at school say you're a welfare kid } \\
\text { and she goes ballistic. } \\
\text { Many of these kids need } \\
\text { She's missing her family }\end{array}$ \\
\hline $\begin{array}{l}\text { Putting into } \\
\text { words for the } \\
\text { child (PW) }\end{array}$ & $\begin{array}{l}\text { Using words to help the } \\
\text { child understand the } \\
\text { problem, understand } \\
\text { themselves, understand } \\
\text { how relationships work } \\
\text { and how families work. }\end{array}$ & $\begin{array}{l}\text { I said to her "kids often feel..." } \\
\text { I would say "We don't need to fight". } \\
\text { I would say "We can sort this out". }\end{array}$ \\
\hline Secure base (SB) & $\begin{array}{l}\text { Being a secure base for } \\
\text { the child, making sure } \\
\text { they feel like they } \\
\text { belong, that they won't } \\
\text { be rejected, that they can } \\
\text { stay living with the } \\
\text { family even when there } \\
\text { are problems. Making } \\
\text { sure they are safe and } \\
\text { that life is predictable. }\end{array}$ & $\begin{array}{l}\text { You will keep on staying with us and we'll } \\
\text { sort this problem out. } \\
\text { You won't get sent away. } \\
\text { I would make sure I was there on time to } \\
\text { pick her up the next day. } \\
\text { I would find a way to let her know I was } \\
\text { thinking about her during the day. }\end{array}$ \\
\hline
\end{tabular}


Example: 'You know, honey, I know you're feeling bad (E) but I'm here to help you and you know we won't send you away (RFC), we sort problems out here and I will help you with it".

This would be coded Empathy (E) and Reflection - foster care (RFC).

Example: "Well, no wonder you felt sad when the teacher called you lazy". (E, RB).

If a response fits more than one category please code as many categories as necessary Example: "She's probably worried she'll be sent away (RFC) so I said to her, families solve problems, you don't get sent away for being naughty". (PW,SB)"

This would be coded Reflection - foster care, Putting it into words for the child and Secure base).

A response is coded for any suggestions made about how to help or helping the child or advice to a foster parents on their own response or helping the child which fits the definitions below. On the rare occasions a suggestion is negative please code that with a $(\mathrm{N})$. Example: "He's been beaten by his Mum so badly that there's no way I can help him to stop bashing kids at school".

Example using the word understand: "We understand" ( E); “We understand it's cause your Nan died “ (E, RB).

If there is no context that the speaker is referring to foster care, for example, "all kids..." then don't code as RFC. 


\section{Appendix E-Practitioner Interview (semi-structured)}

Part 1: I will be asking you a number of questions about yourself and your work If you don't want to answer any questions just say so and if you wish to stop the interview at any point, please let me know. .

\begin{tabular}{|l|l|}
\hline Age & \\
\hline Gender & \\
\hline Ethnicity & \\
\hline Qualifications & \\
\hline Place of work & \\
\hline How long have you worked there? & \\
\hline peopleyou work with? & \\
\hline Why are they referred to your service? & \\
\hline Wour work? & \\
\hline What is your role? & \\
\hline What supervision do you have? & \\
\hline
\end{tabular}




\section{Appendix F-Coding Scheme: Study 3: Practitioner Diaries}

\begin{tabular}{|c|c|c|}
\hline Model & $\begin{array}{l}\text { Model } \\
\text { Advantages of } \\
\text { model/training }\end{array}$ & $\begin{array}{l}\text { Any mention of the Relational Learning Framework - } \\
\text { the model, the module, the framework, the relational } \\
\text { exercise, the RLF. } \\
\text { Example: "I used the model...". } \\
\text { Any reference to the advantages of the model and how } \\
\text { the model has helped them in their work. } \\
\text { Example: "Helpful to summarise the history in one } \\
\text { place". }\end{array}$ \\
\hline Attachment & Attachment theory & $\begin{array}{l}\text { Any mention of applying, thinking about, remembering } \\
\text { more or using attachment theory. } \\
\text { Example: "I use my attachment lens more". }\end{array}$ \\
\hline Skills & $\begin{array}{l}\text { Empathy and } \\
\text { validation }\end{array}$ & $\begin{array}{l}\text { Any reference to understanding the child or the child's } \\
\text { behaviour more. Using a functional analysis of } \\
\text { behaviour, working out the causes of behaviour. } \\
\text { Example: "I have a greater understanding". } \\
\text { Example: "I worked out he was missing his Mum so I } \\
\text { helped the foster parent..." } \\
\text { Using empathy and validation more, helping the foster } \\
\text { parent or team members to do so. Using emotion } \\
\text { coaching, helping foster parents think about the child's } \\
\text { perspective, thinking about the child's perspective, } \\
\text { understanding the child's point of view etc. Example: } \\
\text { "Emotion coaching is useful in my role as a social } \\
\text { worker". } \\
\text { Example: "I've been thinking about the foster parent's } \\
\text { ability to empathise. } \\
\text { Any mention of skills gained in the training course } \\
\text { from the behaviour". }\end{array}$ \\
\hline
\end{tabular}




\begin{tabular}{|l|l|l|}
\hline Difficulties & $\begin{array}{l}\text { Difficulties with } \\
\text { model/training }\end{array}$ & $\begin{array}{l}\text { Any difficulties implementing the training or model. } \\
\text { Example:" I found it a challenge to complete in } \\
\text { isolation". }\end{array}$ \\
& Negative changes & $\begin{array}{l}\text { Any reference to negative outcomes from using the } \\
\text { model and how people responded. } \\
\text { Example: "The client didn't like that idea". }\end{array}$ \\
\hline Positives & $\begin{array}{l}\text { Positive changes } \\
\text { Increased } \\
\text { awareness/mindful }\end{array}$ & $\begin{array}{l}\text { Any reference to positive outcomes from using the } \\
\text { model, how people responded } \\
\text { Example: "Parents appreciated the practical approach". } \\
\text { Being mindful of this information, being aware, gaining } \\
\text { insight. } \\
\text { Example: "I was more mindful of my words". }\end{array}$ \\
\hline
\end{tabular}

Notes. Count "I need to" statements. There is some overlap, for example, "better joint understanding" would be coded as "understanding" and "positive change". Example: "I can see how the framework model makes the relationship less scary" would be coded as "model" and "advantages of model/training". 


\section{References}

Aarons, G.A., Brown, S.A., Hough, R.L., Garland, A.F., \& Wood, P.A. (2001). Prevalence of adolescent substance use disorders across five sectors of care. Journal of the American Academy of Child and Adolescent Psychiatry, 40, 419-426. doi:10.1097/00004583-200104000-00010

Aber, J.L., Slade, A., Berger, B., Bresgi, I., \& Kaplan, M. (1985). The Parent Development Interview. New York NY: Barnard College, Department of Psychology.

Achenbach, T.M., Becker, A., Döpfner, M., Heiervang, E., Roessner, V., Steinhausen, H.C., \& Rothenberger, A. (2008). Multicultural assessment of child and adolescent psychopathology with ASEBA and SDQ instruments: Research findings, applications, and future directions. Journal of Child Psychology and Psychiatry and Allied Disciplines 49, 251-275. doi:10.1111/j.1469-7610.2007.01867.x

Achenbach, T., \& Rescorla, L. (2000). Manual for ASEBA Preschool Form and Profiles. Burlington, VT: University of Vermont.

Ackerman, J.P., \& Dozier, M. (2005). The influence of foster parent investment on children's representations of self and attachment figures. Journal of Applied Developmental Psychology, 26, 507-520. doi:10.1016/j.appdev.2005.06.003

Ai, A.L., Foster, L.J.J., Pecora, P.J., Delaney, N., \& Rodriguez, W. (2013). Reshaping Child Welfare's response to trauma: Assessment, evidence-based intervention, and new research perspectives. Research on Social Work Practice, 23, 651-668. doi:10.1177/1049731513491835

Ainsworth, M.D.S., Blehar, M.C., Waters, E., \& Wall, S. (1978). Patterns of attachment: A psychological study of the strange situation. Hillsdale, NJ: Erlbaum.

Allen, J., \& Vostanis P. (2005). The impact of abuse and trauma on the developing child: An evaluation of a training programme for foster carers and supervising social workers. Adoption \& Fostering, 29, 68 - 81. doi:10.1177/030857590502900308

Alliger, G.M., Tannenbaum, S.I., Bennett, W. Jr., Traver, H., \& Shotland, A. (1997). A meta-analysis of relations among training criteria. Personnel Psychology, 50, 341-358. doi:10.1111/j.1744-6570.1997.tb00911.x 
Alpert, L.T., \& Britner, P.A. (2005). Social workers' attitudes toward parents of children in child protective services: Evaluation of a family-focused casework training program. Journal of Family Social Work, 9, 33-36. doi:10.1300/j039v09n01_03

Altenhofen, S., Clyman, R., Little, C., Baker, M., \& Biringen, Z. (2013). Attachment security in three-year-olds who entered substitute care in infancy. Infant Journal of Mental Health, 34, 435-445. doi:10.1002/imhj.21401

American Psychiatric Association. (2000). Diagnostic and statistical manual of mental disorders (4th ed., text rev.). Washington, DC: American Psychiatric Association doi:10.1176/appi.books.9780890423349.

American Psychiatric Association (2013). Diagnostic and statistical manual of mental disorders $\left(5^{\text {th }}\right.$ ed.). Washington, DC: American Psychiatric Association. doi:10.1176/appi.books.9780890425596.893619

Anctil, T.M., McCubbin, L.D., O’Brien, K., \& Pecora, P. (2007). An evaluation of recovery factors for foster care alumni with physical or psychiatric impairments: Predictors of psychological outcomes. Children and Youth Services Review, 29, 1021-1034. doi:10.1016/j.childyouth.2007.02.003

Anderson, M., \& Linares, L.O. (2012). The role of cultural dissimilarity factors on child adjustment following foster placement. Children and Youth Services Review, 34, 597601. doi:10.1016/j.childyouth.2011.11.016

Antle, B. F., Barbee, A.P., \& van Zyl, M.A. (2008). A comprehensive model for child welfare training evaluation, Children and Youth Services Review, 30, 1063-1080. doi:10.1016/j.childyouth.2008.02.002

Appleyard, K., Egeland, B., van Dulmen, M.H.M., \& Sroufe, L.A. (2005). When more is not better: The role of cumulative risk in child behavior outcomes. Journal of Child Psychology and Psychiatry and Allied Disciplines, 46, 235-245. doi:10.1111/j.14697610.2004.00351.x

Arnott, B., \& Meins, E. (2008). Links among antenatal attachment representations, postnatal mind-mindedness, and infant attachment security: A preliminary study of mothers and 
fathers. Bulletin of the Menninger Clinic, 71, 132-149.

doi:10.1521/bumc.2007.71.2.132

Atukpawu, G., Mertinko, E., Graham, E., \& Denniston, J.J. (2012). Supervisor training to support principle-driven practice with youth in foster care. Children and Youth Services Review, 34, 680-690. doi:10.1016/j.childyouth.2011.12.015

Atwool, N. (2010). Children in care: A report into the quality of services provided to children in care. Office of the Children's Commissioner. Retrieved from: http://www.occ.org.nz

Australian Institute of Health and Welfare, (2013). Child protection Australia 2011-2012 (Child welfare series, no. 55. Cat. No. CWS 43). Retrieved from: http://www.aihw. gov.au/publication-detail/?id=60129542755

Aviezer, O., Sagi, A., Joels, T., \& Ziv, Y. (1999). Emotional availability and attachment representations in kibbutz infants and their mothers. Developmental Psychology, 35, 811-821. doi:10.1037//0012-1649.35.3.811

Barber, J.G., \& Delfabbro, P.H. (2004). Children in foster care. London: Routledge.

Barber, J., \& Delfabbro, P. ( 2005). Children's adjustment to long-term foster care. Children and Youth Services Review, 27, 329-340. doi:10.1016/j.childyouth.2004.10.010

Barber, J.G., Delfabbro, P.H., \& Cooper, L.L. (2001). The predictors of unsuccessful transition to foster care. Journal of Child Psychology and Psychiatry and Allied Disciplines 42, 785-790. doi:10.1111/1469-7610.00775

Barcons, N., Abrines, N., Brun, C., Sartini, C., Fumadó, V., \& Marre, D. (2014). Attachment and adaptive skills in children of international adoption. Child and Family Social Work, 19, 89-98. doi:10.1111/j.1365-2206.2012.00883.x

Barkley, R.A., Edwards, G., Laneri, M., Fletcher, K., \& Metevia, L. (2001). The efficacy of problem-solving communication training alone, behavior management training alone, and their combination for parent-adolescent conflict in teenagers with ADHD and ODD. Journal of Consulting and Clinical Psychology, 69, 926-941. doi:10.1037//0022-006x.69.6.926 
Barlow, D.H., Nock, M.K., \& Hersen, M. (2009). Single case experimental designs: Strategies for studying behaviour change ( $3^{\text {rd }}$ ed.). Boston, MA: Allyn and Bacon.

Barth, R.P., Wildfire, J., \& Green, R.L. (2006). Placement into foster care and the interplay of urbanicity, child behavior problems, and poverty. American Journal of Orthopsychiatry, 76, 358-366. doi:10.1037/0002-9432.76.3.358

Bates, B., \& Dozier, M. (1998). "This Is My Baby Interview” and Coding Manual. Unpublished manuscript, University of Delaware, Newark.

Bates, B., \& Dozier, M. (2005). “This Is My Baby Interview” and Coding Manual. Unpublished manuscript, University of Delaware, Newark, NJ.

Beautrais, A.L. (2001). Child and young adolescent suicide in New Zealand. Australian and New Zealand Journal of Psychiatry, 35, 647-653. doi:10.1046/j.14401614.2001.00944.x

Beck, A. T. (1976). Cognitive therapy and the emotional disorders. New York NY: International University Press.

Bell, T., Romano, E., \& Flynn, R.J. (2013). Multilevel correlates of behavioural resilience among children in child welfare. Child Abuse and Neglect, 37, 1007-1020. doi:10.1016/j.chiabu.2013.07.005

Bellamy, J.L., Gopalan, G., \& Traube, D.E. (2010). A national study of the impact of outpatient mental health services for children in long-term foster care. Clinical Child Psychology and Psychiatry, 15, 467-479. doi:10.1177/1359104510377720

Belsky, J., Bakermans-Kranenburg, M.J., \& van IJzendoorn, M.H. (2007). For better and for worse: Differential susceptibility to environmental influences. Current Directions in Psychological Science, 16, 300 - 304. doi:10.1111/j.1467-8721.2007.00525.x

Benoit, D., Parker, K.C.H., \& Zeanah, C.H. (1997). Mothers' representations of their infants assessed prenatally: Stability and association with infants' attachment classifications. Journal of Child Psychology and Psychiatry and Allied Disciplines, 38, 307-313. doi:10.1111/j.1469-7610.1997.tb01515.x 
Berger, L.M., Bruch, S.K., Johnson, E.I., James, S., \& Rubin, D. (2009). Estimating the "impact" of out-of-home placements on child well-being: Approaching the problem of selection bias. Child Development, 80, 1856-1876. doi:10.1111/j.14678624.2009.01372.x

Bernard, K., Butzin-Dozier, Z., Rittenhouse, J., \& Dozier, M. (2010). Cortisol production patterns in young children living with birth parents vs children placed in foster care following involvement of Child Protective Services. Archives of Pediatric and Adolescent Medicine, 164, 438-443. doi:10.1001/archpediatrics.2010.54

Bernard, K., \& Dozier, M. (2011). This is my baby: Foster parents' feelings of commitment and displays of delight. Infant Mental Health Journal, 32, 251-262. doi:10.1002/imhj.20293

Bernard, K., Dozier, M., Bick, J., Lewis-Morrarty, E., Lindhiem, O., \& Carlson, E. (2012). Enhancing attachment organization among maltreated children: Results of a randomized clinical trial. Child Development, 83, 623-636. doi:10.1111/j.14678624.2011.01712.x

Bernier, A., Ackerman, J.P., \& Stovall-McClough, K.C. (2004). Predicting the quality of attachment relationships in foster care dyads from infants' initial behaviors upon placement. Infant Behavior and Development, 27, 366-381. doi:10.1016/j.infbeh.2004.01.001

Bernier, A., Carlson, S.M., \& Whipple, N. (2010). From external regulation to selfregulation: Early parenting precursors of young children's executive functioning. Child Development, 81, 326-339. doi:10.1111/j.1467-8624.2009.01397.x

Bernier, A., \& Dozier, M. (2003). Bridging the attachment transmission gap: The role of maternal mind-mindedness. International Journal of Behavioral Development, 27, 355365. doi:10.1080/01650250244000399

Berzin, S.C. (2008). Difficulties in the transition to adult-hood: Using propensity scoring to understand what makes foster youth vulnerable. Social Service Review, 82, 171-195. doi:10.1086/588417 
Biehal, N., Ellison, S., Baker, C., \& Sinclair, I. (2011). Outcomes in Long Term Foster Care and Adoption. Adoption Research Initiative. www.adoptionresearchinitiative.org.uk.

Biehal, N., Ellison, S., \& Sinclair, I. (2011). Intensive fostering: An independent evaluation of MTFC in an English setting. Children and Youth Services Review, 33, 2043-2049. doi:10.1016/j.childyouth.2011.05.033

Biringen, Z. (2000). Emotional availability: Conceptualization and research findings. American Journal of Orthopsychiatry, 70, 104-114. doi:10.1037/h0087711

Biringen, Z., (2008). The EA Professionals and EA Parent Curricular. http://www.emotionalavailability.com.

Biringen, Z., Damon, J., Grigg, W., Mone, J., Pipp-Siegel, S., Skillern, S., \& Stratton, J. (2005). Emotional availability: Differential predictions to infant attachment and kindergarten adjustment based on observation time and context. Infant Mental Health Journal, 26, 295-308. doi:10.1002/imhj.20054

Biringen, Z., \& Robinson, J. (1991). Emotional availability in mother-child interactions: A reconceptualization for research. American Journal of Orthopsychiatry, 61, 258-271. doi: $10.1037 / \mathrm{h} 0079238$

Biringen, Z., Skillern, S., Mone, J., \& Pianta, R. (2005). Emotional availability is predictive of the emotional aspects of children's "school readiness". Journal of Early Childhood and Infant Psychology, 1, 89-97.

Blampied, N. M. (1999). A legacy neglected: Restating the case for single-case research in cognitive-behaviour therapy. Behaviour Change, 16, 89-104. doi:10.1375/bech.16.2.89

Blampied, N.M. (2001). The third way: Single case research, training and practice in clinical psychology. Australian Psychologist, 36, 157-163. doi:10.1080/00050060108259648

Blampied, N.M. (2013). Using modified Brinley plots to analyse behaviour change in individuals within groups. Unpublished manuscript. Department of Psychology, University of Canterbury, Christchurch, New Zealand. 
Bouchard, M-A., Target, M., Lecours, S., Fonagy, P., Tremblay, L-M., Schachter, A., \& Stein, H. (2008). Mentalization in adult attachment narratives: Reflective functioning, mental states, and affect elaboration compared. Psychoanalytic Psychology, 25, 47-66. doi:10.1037/0736-9735.25.1.47

Bourdon, K.H., Goodman, R., Rae, D.S., Simpson, G., \& Koretz, D.S. (2005). The Strengths and Difficulties Questionnaire: U.S. normative data and psychometric properties. Journal of the American Academy of Child and Adolescent Psychiatry, 44, 557-564. doi:10.1097/01.chi.0000159157.57075.c8

Bowlby, J. (1969/1982). Attachment and Loss. Vol 1: Attachment ( $2^{\text {nd }}$ ed.) New York NY: Basic Books.

Bowlby, J. (1973). Attachment and Loss: Vol. 2. Separation: Anxiety and anger. New York NY: Basic Books.

Brannan, A. M., Heflinger, C. A., \& Bickman, L. (1997). The Caregiver Strain Questionnaire: Measuring the impact on the family of living with a child with serious emotional problems. Journal of Emotional and Behavioral Disorders, 5, 212-222. doi:10.1177/106342669700500404

Braun, V., \& Clarke, V. (2006). Using thematic analysis in psychology. Qualitative Research in Psychology, 3, 77-101. doi:10.1191/1478088706qp063oa

Breslau, N., Koenen, K.C., Luo, Z., Agnew-Blais, J., Swanson, S., Houts, R.M.,...Moffitt, T.E. (2014). Childhood maltreatment, juvenile disorders and adult post-traumatic stress disorder: A prospective investigation. Psychological Medicine, 44, 1937-1945. doi:10.1017/s0033291713002651

Bretherton, I. (2000). Emotional availability: an attachment perspective. Attachment and Human Development, 2, 233-241. doi:10.1080/14616730050085581

Bretherton, I., \& Munholland, K.A. (2008). Internal working models in attachment relationships: Elaborating a central construct in attachment theory. In J. Cassidy \& P.R. Shaver (Eds.), Handbook of attachment: Theory, research and clinical applications (2nd ed., pp. 102-127). New York NY: Guilford. 
Briskman, J., Castle, J., Blackeby, K., Bengo, C., Slack, K., Stebbens, C., Leaver, W., \& Scott, S. (2012). Randomised Controlled Trial of the Fostering Changes Programme. Report to Department for Education. London, UK.

Brown, D.W., Anda, R.F., Tiemeier, H., Felitti, V.J., Edwards, V.J., Croft, J.B., \& Giles, W.H. (2009). Adverse childhood experiences and the risk of premature mortality. American Journal of Preventive Medicine, 37, 389-396.

doi:10.1016/j.amepre.2009.06.021

Bruce, J., Fisher, P.A., Pears, K.C., \& Levine, S. (2009). Morning cortisol levels in preschool-aged foster children: Differential effects of maltreatment type.

Developmental Psychobiology, 51, 14-23. doi:10.1002/dev.20333

Buehler, C., Rhodes, K., Orme, J., \& Cuddeback, G. (2006). The potential for successful foster family care: Conceptualizing competency domains for foster parents. Child Welfare, 85, 523-558.

Burns, B.J., Phillips, S.D., Wagner, H.R., Barth, R.P., Kolko, D.J., Campbell, Y., \& Landsverk, J. (2004). Mental health need and access to mental health services by youths involved with child welfare: A national survey. Journal of the American Academy for Child and Adolescent Psychiatry, 43, 960-970.

doi:10.1097/01.chi.0000127590.95585.65

Bywater, T., Hutchings, J., Linck, P., Whitaker, C., Daley, D., Yeo S. T., \& Edwards R. T. (2011). Incredible Years parent training support for foster carers in Wales: A multicentre feasibility study. Child: Care, Health and Development, 37, 233-243. doi:10.1111/j.1365-2214.2010.01155.x

Calvert, S. (2000). Severe needs project: Review of service packages. Tauranga, New Zealand: Department of Child, Youth and Family Services.

Carlson, E. (1998). A prospective longitudinal study of attachment disorganization/disorientation. Child Development, 69, 1970-1979. doi:10.1111/j.14678624.1998.tb06163.x 
Carnes-Holt, K., \& Bratton, S.C. (2014). The efficacy of child parent relationship therapy for adopted children with attachment disruptions. Journal of Counseling and Development, 92, 328-337. doi:10.1002/j.1556-6676.2014.00160.x

Carpenter, J., Patsios, D., Szilassy, E., \& Hackett, S. (2011). Outcomes of short course interprofessional education in parental mental illness and child protection: Selfefficacy, attitudes and knowledge. Social Work Education, 30, 195-206. doi:10.1080/02615479.2011.540394

Caspi, A., \& Moffitt, T.E. (2006). Gene-environment interactions in psychiatry: Joining forces with neuroscience. Nature Reviews Neuroscience, 7, 583-590. doi:10.1038/nrn1925

Cassidy, J., Lichtenstein-Phelps, J., Sibrava, N.J., Thomas, C.L., \& Borkovec, T.D. (2009). Generalized Anxiety Disorder: Connections with self-reported attachment. Behavior Therapy,40, 23-38. doi:10.1016/j.beth.2007.12.004.

Chamberlain P. (2003). The Oregon multidimensional treatment foster care model: features, outcomes, and progress in dissemination. Cognitive and Behavioral Practice, 10, 30312. doi:10.1016/s1077-7229(03)80048-2

Chamberlain, P., Leve, L.D., \& DeGarmo, D.S. (2007). Multidimensional treatment foster care for girls in the juvenile justice system: 2-Year follow-up of a randomized clinical trial. Journal of Consulting and Clinical Psychology, 75,187-193. doi:10.1037/0022006x.75.1.187

Chamberlain, P., Price, J., Leve, L.D., Laurent, H., Landsverk, J.A., \& Reid, J B. (2008). Prevention of behavior problems for children in foster care: Outcomes and mediation effects. Prevention Science, 9, 17-27. doi:10.1007/s11121-007-0080-7

Chamberlain, P., Price, J.M., Reid, J.B., Landsverk, J., Fisher, P.A., \& Stoolmiller, M. (2006). Who disrupts from placement in foster and kinship care? Child Abuse and Neglect, 30, 409-424. doi:10.1016/j.chiabu.2005.11.004

Chamberlain, P., \& Reid, J.B. (1987). Parent observation and report of child symptoms. Behavioral Assessment, 9, 97-109. doi:10.1007/s10826-006-9029-х 
Cheung, C., Goodman, D., Leckie, G., \& Jenkins, J.M. (2011). Understanding contextual effects on externalizing behaviors in children in out-of-home care: Influence of workers and foster families. Child and Youth Services Review, 33, 2050-2060. doi:10.1016/j.childyouth.2011.05.036

Child, Youth and Family (2006). EXG Review: Sustainability of the Care and Protection System: Permanency. 6 November 2006. Retrieved from: http://www.treasury.govt.nz/ publications/informationreleases/exgreviews/pdfs/cyf-msd-exgrev-perm-nov06.pdf

Child, Youth and Family (2012). Child Youth and Family Strategic Plan, 2012-2015. Retrieved from: http://www.cyf.govt.nz/about-us/who-we-are-what-we-do/informationfor-media-backup.html

Child, Youth and Family, (2014). Key Statistics and information for media. Retrieved fromhttp://www.cyf.govt.nz/about-us/who-we-are-what-we-do/information-for-mediabackup.html.

Cicchetti, D. (2010). Resilience under conditions of extreme stress: A multilevel perspective. World Psychiatry, 9, 145-154.

Cicchetti, D., \& Lynch, M. (1993). Toward an ecological/transactional model of community violence and child maltreatment: Consequences for children's development. Psychiatry, 56, 96-118.

Cicchetti, D., Rogosch, F.A., \& Toth, S.L. (2006). Fostering secure attachment in infants in maltreating families through preventive interventions. Development and Psychopathology, 18, 623-649. doi:10.1017/s0954579406060329

Cicchetti, D. \& Toth, S.L. (2014). A multilevel perspective on child maltreatment. In M. Lamb \& C.Garcia Coll (Eds.), Handbook of child psychology and developmental science: Vol 3. Socioemotional process ( $7^{\text {th }}$ ed.). Hoboken, N.J: Wiley.

Cicchetti, D., \& Valentino, K. (2006). An ecological-transactional perspective on child maltreatment: Failure of the average expectable environment and its influence on child development. In D. Cicchetti \& D. J. Cohen (Eds.), Developmental psychopathology, Vol. 3: Risk, disorder and adaptation (pp. 129-210). Hoboken, NJ: Wiley. 
Clark, T.C., Robinson, E., Crengle, S., Grant, S., Galbreath, R.A., \& Sykora, J. (2009). Youth '07: The health and wellbeing of secondary school students in New Zealand: Findings on young people and violence. Auckland: University of Auckland.

Cohen, J.A., Mannarino, A.P., \& Deblinger, E. (2006). Treating trauma and traumatic grief in children and adolescents. New York NY: Guilford.

Cole, S.A. (2005). Foster caregiver motivation and infant attachment: How do reasons for fostering affect relationships? Child and Adolescent Social Work Journal, 22, 441-457. doi:10.1007/s10560-005-0021-x

Colonnesi, C., Wissink, I.B., Noom, M.J., Asscher, J.J., Hoeve, M., Stams, G.J.J.M.,... Kellaert-Knol, M.G. (2012). Basic Trust: An Attachment-Oriented Intervention Based on Mind-Mindedness in Adoptive Families. Research on Social Work Practice, 23, 179-188. doi:10.1177/1049731512469301

Conn, A.-M., Szilagyi, M.A., Franke, T.M., Albertin, C.S., Blumkin, A.K., \& Szilagyi, P.G. (2013). Trends in child protection and out-of-home care. Pediatrics, 132, 712-719. doi:10.1542/peds.2013-0969d

Connolly, M., Wells, P., \& Field, J. (2007). Working with vulnerable infants. Social Work Now, 38, 5-10.

Conners-Burrow, N.A., Kramer, T.L., Sigel, B.A., \& Helpenstill, K. (2013). Traumainformed care training in a child welfare system: Moving it to the front line. Children and Youth Services Review, 35, 1830-1835. doi:10.1016/j.childyouth.2013.08.013

Cornish, A.M., McMahon, C., \& Ungerer, J. (2008). Postnatal depression and the quality of mother-infant interactions during the second year of life. Australian Journal of Psychology, 60, 142-151. doi:10.1080/00049530701477738

Cortina, J. M. (1993). What Is coefficient alpha? An examination of theory and applications. Journal of Applied Psychology, 78, 98-104. doi:10.1037//0021-9010.78.1.98 
Christenson, B.L., \& McMurtry, J. (2009). A longitudinal evaluation of the preservice training and retention of kinship and nonkinship foster/adoptive families one and a half years after training. Child Welfare, 88, 5-22.

Craven, P., \& Lee, R. (2006). Therapeutic interventions for foster children: A systematic research synthesis. Research on Social Work Practice, 16, 287-304. doi:10.1177/1049731505284863

Crosland, K.A., Dunlap, G., Sager, W., Neff, B., Wilcox, C., Blanco, A., \& Giddings, T. (2008). The effects of staff training on the types of interactions observed at two group homes for foster care children. Research on Social Work Practice, 18, 410-420. doi:10.1177/1049731507314000

Curry, D., \& Chandler, T. (1999). The Human Services Training Effectiveness Postcard (HSTEP): A Tool for Research and Evaluation of Human Services Training. Professional Development: The International Journal of Continuing Social Work Education, 2, 43-56.

Cyr, C., Euser, E.M., Bakermans-Kranenburg, M. J., \& van IJzendoorn, M. H. (2010). Attachment security and disorganization in maltreating and high-risk families: A series of meta-analyses. Development and Psychopathology, 22, 87-108. http://dx.doi:10.1017/s0954579409990289

Dallos, R., Morgan-West, K., \& Denman, K. (2014). Changes in attachment representations for young people in long-term therapeutic foster care. Clinical Child Psychology and Psychiatry. Advance online publication. doi:10.1177/1359104514543956

Danese, A., Moffitt, T.E., Harrington, H., Milne, B.J., Polanczyk, G., Pariante, C.M., ... Caspi, A. ( 2009). Adverse childhood experiences and adult risk factors for age-related disease. Archives of Pediatric and Adolescent Medicine, 163,1135-1143. doi:10.1001/archpediatrics.2009.214

Deblinger, E., \& Heflin, A.H. (1996). Treating sexually abused children and their nonoffending parents: A cognitive behavioural approach. Thousand Oaks, CA: Sage Publications. 
DeGarmo, D.S., Reid, J.B., Fetrow, B.A., Fisher, P.A., \& Antoine, K.D. (2013). Preventing child behavior problems and substance use: The pathways home foster care reunification intervention. Journal of Child and Adolescent Substance Abuse, 22, 388406. doi:10.1080/1067828x.2013.788884

Demers, I., Bernier, A., Tarabulsy, G.M., \& Provost, M.A. (2010a). Mind-mindedness in adult and adolescent mothers: Relations to maternal sensitivity and infant attachment. International Journal of Behavioral Development, 34, 529-537. doi:10.1177/0165025410365802

Demers, I., Bernier, A., Tarabulsy, G.M., \& Provost, M.A. (2010b). Maternal and child characteristics as antecedents of maternal mind-mindedness. Infant Mental Health Journal, 31, 94-112. doi:10.1002/imhj.20244

Dorsey, S., Farmer, E.M.Z., Barth, R.P., Greene, K.M., Reid, J., \& Landsverk, J. (2008). Current status and evidence base of training for foster and treatment foster parents. Children and Youth Services Review, 30, 1403- 1416. doi:10.1016/j.childyouth.2008.04.008

Dorsey, S., Pullmann, M.D., Berliner, L., Koschmann, E., McKay, M., \& Deblinger, E. (2014). Engaging foster parents in treatment: A randomized trial of supplementing trauma-focused cognitive behavioral therapy with evidence-based engagement strategies. Child Abuse and Neglect, 38, 1508-1520. doi:10.1016/j.chiabu.2014.03.020

Dozier, M., \& Lindhiem, O. (2006). This Is My Child: Differences among foster parents in commitment to their young children. Child Maltreatment, 11, 338-345. doi:10.1177/1077559506291263

Dozier, M., Lindhiem, O., Lewis, E., Bernard, K., \& Peloso, E. (2009). Effects of a foster parent training program on young children's attachment behaviors: Preliminary evidence from a randomized clinical trial. Child and Adolescent Social Work Journal, 26, 321-332. doi:10.1007/s10560-009-0165-1

Dozier, M., Peloso, E., Lewis, E., Laurenceau, J-P., \& Levine, S. (2008). Effects of an attachment-based intervention on the cortisol production of infants and toddlers in 
foster care. Development and Psychopathology, 20, 845-859.

doi:10.1017/s0954579408000400

Dozier, M., Peloso, E., Lindhiem, O., Gordon, M.K., Manni, M., Sepulveda, S., ...Levine, S. (2006). Developing evidence-based interventions for foster children: An example of a randomized clinical trial with infants and toddlers. Journal of Social Issues. 62, 767785. doi:10.1111/j.1540-4560.2006.00486.x

Dozier, M., Stovall, K., Albus, K., \& Bates, B. (2001). Attachment for infants in foster care: The role of caregiver state of mind. Child Development, 72, 1467-1477. doi:10.1111/1467-8624.00360

Dugmore, P., \& Cocker, C. (2008). Legal, social and attitudinal changes: An exploration of lesbian and gay issues in a training programme for social workers in fostering and adoption. Social Work Education, 27, 159-168. doi:10.1080/02615470701709600

Duncan, B. L., Sparks, J., Miller, S. D., Bohanske, R., \& Claud, D. (2006). Giving youth a voice: A preliminary study of the reliability and validity of a brief outcome measure for children, adolescents, and caretakers. Journal of Brief Therapy, 5, 66-82.

Dweck, C.S., \& London, B. (2004). The role of mental representation in social development. Merrill-Palmer Quarterly, 50, 428-444. doi:10.1353/mpq.2004.0029

Eapen, V. (2014). Developmental and mental health disorders: Two sides of the same coin. Asian Journal of Psychiatry, 8, 7-11. doi:10.1016/j.ajp.2013.10.007

Easterbrooks, M. A., Biesecker, G., \& Lyons-Ruth, K., (2000). Infancy predictors of emotional availability in middle childhood: The roles of attachment security and maternal depressive symptomatology. Attachment and Human Development, 2, 170187. doi:10.1080/14616730050085545

Easterbrooks, M.A., Chauduri, J.H., \& Gestsdottir, S. (2005). Patterns of emotional availability among young mothers and their infants: A dyadic, contextual analysis. Infant Mental Health Journal, 26, 309-326. doi:10.1002/imhj.20057

Eddy, J.M., Bridges, R., Whaley, R.B., \& Chamberlain, P. (2004). The Prevention of Violent Behavior by Chronic and Serious Male Juvenile Offenders: A 2-Year Follow-up of a 
Randomized Clinical Trial. Journal of Emotional and Behavioral Disorders, 12, 2-8. doi:10.1177/10634266040120010101

English, D.J., Upadhyaya, M.P., Litrownik, A.J., Marshall, J.M., Runyan, D.K., Graham, J.C., \& Dubowitz, H. (2005). Maltreatment's wake: The relationship of maltreatment dimensions to child outcomes. Child Abuse and Neglect, 29, 597 - 619. doi:10.1016/j.chiabu.2004.12.008

Ereky-Stevens, K. (2008). Associations between mothers' sensitivity to their infants' internal states and children's later understanding of mind and emotion. Infant and Child Development, 17, 527-543. doi:10.1002/icd.572

Escobar, M.J., \& Santelices, M.P. (2013). Attachment in adopted adolescents. National adoption in Chile. Children and Youth Services Review, 35, 488-492. Doi:10.1016/j.childyouth.2012.12.011

Everson-Hock, E.S., Jones, R., Guillaume, L., Clapton, J., Goyder, E., Chilcott, J., . . . Swann, C. (2011). The effectiveness of training and support for carers and other professionals on the physical and emotional health and well-being of looked-after children and young people: a systematic review. Child: Care, Health and Development, 38, 162-174. doi:10.1111/j.1365-2214.2011.01247.x

Every Child Counts (2010). The nature of economic costs from child abuse and neglect in New Zealand. ECC Discussion paper No 1, June. Retrieved from: http://www. infometrics.co.nz/ reports/ ECC-Child-Abuse-Neglect-FULL-REPORT.pdf

Eyberg, S. M., \& Funderbunk, B. W. (2011). Parent-child Interaction Therapy Protocol. Gainesville, FL: PCIT International.

Farmer, E.M.Z., Burns, B.J., Wagner, H. R., Murray, M., \& Southerland, D. (2010). Enhancing "usual practice" treatment foster care: Findings from a randomized trial on improving youths' outcomes. Psychiatric Services, 61, 555 - 561. doi:10.1176/appi.ps.61.6.555

Farmer, E.M.Z., Mustillo, S.A., Wagner, H.R., Burns, B.J., Kolko, D.J., Barth, R.P., \& Leslie, L.K.(2010). Service use and multi-sector use for mental health problems by 
youth in contact with child welfare. Children and Youth Services Review, 32, 815-821. doi:10.1016/j.childyouth.2010.01.019

Farrow, C., \& Blissett, J. (2014). Maternal mind-mindedness during infancy, general parenting sensitivity and observed child feeding behavior: A longitudinal study. Attachment and Human Development, 16, 230-241. doi:10.1080/14616734.2014.898158

Fearon, R.P., Bakermans-Kranenburg, M. J., van IJzendoorn, M.H., Lapsley, A.M., \& Roisman, G.I. (2010). The significance of insecure attachment and disorganization in the development of children's externalizing behavior: A meta-analytic study. Child Development, 81, 435-456. doi:10.1111/j.1467-8624.2009.01405.x

Feather, J.S., \& Ronan, K.R. (2009). Trauma-focused CBT with maltreated children: A clinic-based evaluation of a new treatment manual. Australian Psychologist, 44, 174194. doi:10.1080/00050060903147083

Fergusson, D.M., Boden, J.M., \& Horwood, L.J. (2012). Moderating role of the MAOA genotype in antisocial behaviour. The British Journal of Psychiatry, 200: 116-123. doi: 10.1192/bjp.bp.111.093328

Fergusson D.M. \& Horwood L. J. (2001). The Christchurch Health and Development Study: Review of findings on child and adolescent mental health. Australian and New Zealand Journal of Psychiatry, 35, 287-296. doi:10.1046/j.1440-1614.2001.00902.x

Fergusson, D.M., Horwood,L. J., \& Lynskey, M.T. (1996). Psychiatric outcomes of childhood sexual abuse. Journal of the American Academy of Child and Adolescent Psychiatry, 35, 1365-1375.

Fisher, P.A., Gunnar, M.R., Dozier, M., Bruce, J., \& Pears, K.C. (2006). Effects of a therapeutic intervention for foster children on behaviour problems, caregiver attachment and stress regulatory neural systems. Annals of the New York Academy of Sciences, 1094, 215-225. doi:10.1196/annals.1376.023

Fisher, P. A., \& Kim, H.K. (2007). Intervention effects on preschoolers' attachment-related behaviors from a randomized trial. Prevention Science, 8, 161-170. doi:10.1007/s11121-007-0066-5 
Fisher, P.A., Kim, H.K., \& Pears, K.C. (2009). Effects of Multidimensional Treatment Foster Care for Preschoolers (MTFC-P) on reducing permanent placement failures among children with placement instability. Children and Youth Services Review, 31,541-546. doi:10.1016/j.childyouth.2008.10.012

Fisher, P.A., \& Stoolmiller, M. (2008). Intervention effects on foster parent stress: Associations with child cortisol levels. Development and Psychopathology, 20,10031021. doi:10.1017/s0954579408000473

Fisher, P.A., Stoolmiller, M., Gunnar, M.R., \& Burraston, B.O. (2007). Effects of a therapeutic intervention for foster preschoolers on diurnal cortisol activity. Psychoneuroendocrinology 32, 892-905. doi:10.1016/j.psyneuen.2007.06.008

Fonagy P., \& Bateman, A. (2008). The development of Borderline Personality Disorder - A mentalizing model. Journal of Personality Disorders, 22, 4-21. doi:10.1521/pedi.2008.22.1.4

Fonagy, P., Gergely, G., \& Target, M. (2007). The parent-infant dyad and the construction of the subjective self. Journal of Child Psychology and Psychiatry and Allied Disciplines $48,288-328$.

Ford, T., Vostanis, P., Meltzer, H, \& Goodman, R. (2007). Psychiatric disorder among British children looked after by local authorities: comparison with children living in private households. British Journal of Psychiatry, 190,319-325. doi:10.1192/bjp.bp.106.025023

Franz, J., \& Woodward, A. (2006). Foster care. Gale Encyclopedia of Children's Health: Infancy through Adolescence. Retrieved from: http://www.encyclopedia. com/topic/ foster_care.aspx

Garland, A.F., Hough, R.L., McCabe, K.M., Yeh, M., Wood, P.A., \& Aarons, G.A. (2001). Prevalence of psychiatric disorders in youths across five sectors of care. Journal of the American Academy of Child and Adolescent Psychiatry, 40, 409-418. doi:10.1177/108705470100500110

Gaviţa, O.A., David, D., Bujoreanu, S., Tiba, A., \& Ionuţiu, D.R. (2012). The efficacy of a short cognitive-behavioral parent program in the treatment of externalizing behavior 
disorders in Romanian foster care children: Building parental emotion-regulation through unconditional self- and child-acceptance strategies. Children and Youth Services Review, 34, 1290-1297. doi:10.1016/j.childyouth.2012.03.001

George, C., \& Solomon, J. (2008). The caregiving system: A behavioural systems approach to parenting. In J. Cassidy \& P.R. Shaver (Eds.), Handbook of Attachment (pp. 698717). New York NY: Guilford Press.

Gilbert, R., Fluke, J., O'Donnell, M., Gonzalez-Izquierdo, A., Brownell, M., Gulliver, P., ... Sidebotham, P. (2012). Child maltreatment: Variation in trends and policies in six developed countries. The Lancet, 379, 758-772. doi:10.1016/S0140-6736(11)61087-8

Gillis-Arnold, R., Crase, S.J., Stockdale, D.F., \& Shelley II, M.C. (1998). Parenting attitudes, foster parenting attitudes, and motivations of adoptive and nonadoptive foster parent trainees. Children and Youth Services Review, 20, 715-732. doi:10.1016/S0190-7409(98)00033-4

Golding, K., (2007). Developing Group-Based Parent Training for Foster and Adoptive Parents. Adoption \& Fostering, 31, 39-48. doi:10.1177/030857590703100306

Golding, K., \& Picken W. (2004). Group Work for Foster Carers Caring for Children with Complex Problems. Adoption \& Fostering, 28, 25-37. doi:10.1177/030857590402800105

Goodman, R., Ford, T., Simmons, H.,Gatward, R., \& Meltzer, H. (2000). Using the Strengths and Difficulties Questionnaire (SDQ) to screen for child psychiatric disorders in a community sample. British Journal of Psychiatry, 177, 534-539. doi:10.1192/bjp.177.6.534

Goodman, R., \& Scott, S. (1999). Comparing the Strengths and Difficulties Questionnaire and the Child Behavior Checklist: Is small beautiful? Journal of Abnormal Child Psychology, 27, 17-24.

Gottman, J.M., \& Declaire, J. (1997). The heart of parenting: How to raise an emotionally intelligent child. New York NY: Simon and Schuster. 
Green, J. M., Biehal, N., Roberts, C., Dixon, J., Kay, C., Parry, E., ... Sinclair, I. (2014). Multidimensional Treatment Foster Care for Adolescents in English care: Randomised trial and observational cohort evaluation. British Journal of Psychiatry, 204, 214-221. doi:10.1192/bjp.bp.113.131466

Gurney-Smith, B., Granger, C., Randle, A., \& Fletcher, J. (2010). 'In Time and in Tune' The Fostering Attachments Group: Capturing sustained change in both caregiver and child. Adoption and Fostering, 34, 50-60. doi:10.1177/030857591003400406

Hansson, K., \& Olsson, M. (2012). Effects of multidimensional treatment foster care (MTFC): Results from a RCT study in Sweden. Children and Youth Services Review, 34, 1929-1936. doi:10.1016/j.childyouth.2012.06.008

Henry, J.D., \& Crawford, J.R. (2005). The short-form version of the Depression, Anxiety Stress Scales (DASS-21): Construct validity and normative data in a large non-clinical sample. British Journal of Clinical Psychology, 44, 227-239. doi:10.1348/014466505x29657

Hesse, E. (2008). The Adult Attachment Interview: Protocol, method of analysis, and empirical studies. In J. Cassidy \& P.R. Shaver (Eds.), Handbook of attachment: Theory, research and clinical applications (2nd ed., pp. 552-598). New York NY: Guilford.

Hillen, T., \& Gafson, L. (2014). Why good placements matter: Pre-placement and placement risk factors associated with mental health disorders in pre-school children in foster care. Clinical Child Psychology and Psychiatry. Advance online publication. doi:10.1177/1359104514530733

Holzer, P. \& Bromfield, L. (2008) NCPASS Comparability of Child Protection Data: Project Report. Retrieved from Australian Institute of Family Studies: https://apps.aifs.gov.au/ cfcaregister/projects/593

Horwitz, S.M., Chamberlain, P., Landsverk, J., \& Mullican, C. (2010). Improving the mental health of children in child welfare through the implementation of evidence-based parenting interventions. Administration and Policy in Mental Health and Mental Health Services Research, 37, 27-39. doi:10.1007/s10488-010-0274-3 
Howe, D. (2011). Attachment across the lifespan: A brief introduction. Hampshire, UK: Palgrave Macmillan.

Howes, C., \& Ritchie, S. (1999). Attachment organizations in children with difficult life circumstances. Development and Psychopathology, 11, 251-268. doi:10.1017/s0954579499002047

Hurlbert, M.S., Chamberlain, P., DeGarmo, D.S., Zhang, J., \& Price, J. (2010). Advancing prediction of foster placement disruption using brief behavioral screening. Child Abuse and Neglect, 34, 917-926. doi:10.1016/j.chiabu.2010.07.003

Huth-Bocks, A.C., Levendosky,A.A., Bogat,G.A., \& van Eye, A. (2004). The impact of maternal characteristics and contextual variables on infant-mother attachment. Child Development, 75, 480-496. doi:10.1111/j.1467-8624.2004.00688.x

Jackson, Y., Gabrielli, J., Fleming, K., Tunno, A.M., \& Makanui, P.K. (2014). Untangling the relative contribution of maltreatment severity and frequency to type of behavioral outcome in foster youth. Child Abuse and Neglect, 38, 1147-1159. doi:10.1016/j.chiabu.2014.01.008

Jacobsen, H., Ivarsson, T., Wentzel-Larsen, T., Smith, L., \& Moe, V. (2014). Attachment security in young foster children: Continuity from 2 to 3 years of age. Attachment and Human Development, 16, 42-57. doi:10.1080/14616734.2013.850102

Jacobson N.S., \& Truax, P. (1991). Clinical significance: A statistical approach to defining meaningful change in psychotherapy research. Journal of Consulting and Clinical Psychology, 59, 12 - 19. doi:10.1037//0022-006x.59.1.12

Johnston, C., \& Mash, E.J. (1989). A measure of parenting satisfaction and efficacy. Journal of Clinical Child Psychology, 18, 167-175. doi:10.1207/s15374424jccp1802_8

Jose, P.E. (2013a). ModGraph-I: A programme to compute cell means for the graphical display of moderational analyses: The internet version, Version 3.0. Victoria University of Wellington, Wellington, New Zealand. Retrieved March, 2014from http://pavlov. psyc.vuw.ac.nz/ paul-jose/modgraph/

Jose, P.E. (2013b). Doing statistical mediation and moderation. New York NY: Guilford. 
Joseph, M.A., O'Connor, T.G., Briskman, J.A.,Maughan, B., \& Scott, S. (2013). The formation of secure new attachments by children who were maltreated: An observational study of adolescents in foster care. Development and Psychopathology 26, 67-80. doi:10.1017/s0954579413000540

Juffer, F., Bakermans-Kranenburg, M.J., \& van IJzendoorn, M.H. (2005). The importance of parenting in the development of disorganized attachment: Evidence from a preventive intervention study in adoptive families. Journal of Child Psychology and Psychiatry and Allied Disciplines, 46, 263-274. doi:10.1111/j.1469-7610.2004.00353.x

Kazdin, A.E. (1980). Research design in clinical psychology. New York NY: Harper and Row.

Kazdin, A.E. (2001). Progression of therapy research and clinical application of treatment require better understanding of the change process. Clinical Psychology: Science and Practice, 8,143-151. doi:10.1093/clipsy.8.2.143

Keane, B. (2014). 'Whāngai - customary fostering and adoption - The custom of whāngai'. Te Ara - the Encyclopedia of New Zealand. Retrieved from: http://www.TeAra. govt. nz/ en/whāngai-customary-fostering-and-adoption/page-1

Keil, V., \& Price, J.M. (2006). Externalizing behavior disorders in child welfare settings: Definition, prevalence, and implications for assessment and treatment. Children and Youth Services Review, 28, 761 - 779. doi:10.1016/j.childyouth.2005.08.006

Keller, T.E., Salazar, A.M., \& Courtney, M.E. (2010). Prevalence and timing of diagnosable mental health, alcohol, and substance use problems among older adolescents in the child welfare system. Children and Youth Services Review, 32, 626-634. doi:10.1016/j.childyouth.2009.12.010

Kelly, W., \& Salmon, K. (2014). Helping foster parents understand the child in their care: A Relational Learning Framework. Clinical Child Psychology and Psychiatry, 19, 535547. doi:10.1177/1359104514524067

Kerr, D.C.R., Leve, L.D., \& Chamberlain, P. (2009). Pregnancy Rates Among Juvenile Justice Girls in Two Randomized Controlled Trials of Multidimensional Treatment 
Foster Care. Journal of Consulting and Clinical Psychology, 77, 588 - 593. doi:10.1037/a0015289

Kerr, D.C.R., DeGarmo, D.S., Leve, L.D., \& Chamberlain, P. (2014). Juvenile justice girl's depressive symptoms and suicidal ideation 9 years after multidimensional treatment foster care. Journal of Consulting and Clinical Psychology,82, 684-693. doi:10.1037/a0036521

Kerr, L., \& Cossar, J. (2014). Attachment Interventions with Foster and Adoptive Parents: A Systematic Review. Child Abuse Review. Advance online publication. doi:10.1002/car.2313

Kessler, M.L., \& Greene, B.F. (1999). Behavior analysis in child welfare: Competency training caseworkers to manage visits between parents and their children in foster care. Research on Social Work Practice, 9, 148-170. doi:10.1177/104973159900900202

Kim, J., \& Cicchetti, D. (2010). Longitudinal pathways linking child maltreatment, emotion regulation, peer relations, and psychopathology. Journal of Child Psychology and Psychiatry, 51, 706-716. doi:10.1111/j.1469-7610.2009.02202.x

Kim, H.K., \& Leve, L.D. (2011). Substance use and delinquency among middle school girls in foster care: A three-year follow-up of a randomized controlled trial. Journal of Consulting and Clinical Psychology, 79, 740-750. doi:10.1037/a0025949

Kinsey, D., \& Schlösser, A. (2012). Interventions in foster and kinship care: A systematic review. Clinical Child Psychology and Psychiatry, 18, 429-463. doi:10.1177/1359104512458204

Lagacé-Séguin, D.G., \& Coplan, R.J. (2005). Maternal emotional styles and child social adjustment: Assessment, correlates, outcomes and goodness of fit in early childhood Social Development, 14, 613-636. doi:10.1111/j.1467-9507.2005.00320.x

Laranjo, J., Bernier, A., \& Meins, E. (2008). Associations between maternal mindmindedness and infant attachment security: Investigating the mediating role of maternal sensitivity. Infant Behavior and Development, 31, 688-695. http://dx.doi:10.1016/j.infbeh.2008.04.008 
Lau, A.S., Leeb, R.T., English, D., Graham, J.C., Briggs, E.C., Brody, K.E., \& Marshall, J.M. (2005). What's in a name? A comparison of methods for classifying predominant type of maltreatment. Child Abuse and Neglect, 29, 533-551. doi:10.1016/j.chiabu.2003.05.005

Laurent, H.K., Gilliam, K.S., Bruce, J., \& Fisher, P.A. (2014). HPA stability for children in foster care: Mental health implications and moderation by early intervention. Developmental Psychobiology, 56, 1406-1415. doi:10.1002/dev.21226

Lawler, M.J. (2008). Maltreated children's emotional availability with kin and non-kin foster mothers: A sociobiological perspective. Children and Youth Services Review, 30, 1131-1143. doi:10.1016/j.childyouth.2008.02.007

Lawrence, C.R., Carlson, E.A., \& Egeland, B. (2006). The impact of foster care on development. Development and Psychopathology, 18, 57-76. doi:10.1017/s0954579406060044

Laybourne, G., Andersen, J., \& Sands J., (2008). Fostering Attachments in Looked after Children: Further Insight into the Group-Based Programme for Foster Carers. Adoption \& Fostering, 32, 64-76. doi:10.1177/030857590803200409

Leathers, S.J. (2006). Placement disruption and negative placement outcomes among adolescents in long-term foster care: The role of behavior problems. Child Abuse and Neglect, 30, 307-324. doi:10.1016/j.chiabu.2005.09.003

Leathers, S.J., Spielfogel, J.E., Gleeson, J.P., \& Rolock, N. (2012). Behavior problems, foster home integration, and evidence-based behavioural interventions: What predicts adoption of foster children? Children and Youth Services Review, 34, 891-899. doi:10.1016/j.childyouth.2012.01.017

Leathers, S.J., Spielfogel, J.E.,McMeel, L.S., \& Atkins, M.S. ( 2011). Use of a parent management training intervention with urban foster parents: A pilot study. Children and Youth Services Review, 33, 1270-1279. doi:10.1016/j.childyouth.2011.02.022

Leenarts, L.E.W., Diehle, J.,Doreleijers, T.A.H., Jansma, E.P.,\& Lindauer, R.J.L. (2013). Evidence-based treatments for children with trauma-related psychopathology as a result 
of childhood maltreatment: A systematic review. European Child and Adolescent Psychiatry, 22, 269-283. doi:10.1007/s00787-012-0367-5

Lehmann, S., Havik, O.E., Havik, T., \& Heiervang, R. ( 2013). Mental disorders in foster children: a study of prevalence, comorbidity and risk factors. Child and Adolescent Psychiatry and Mental Health, 7:39. doi:10.1186/1753-2000-7-39

Lesch, E., Deist, M., Booysen, L., \& Edwards, E. (2013). South African social workers' knowledge of attachment theory and their perceptions of attachment relationships in foster care supervision. Children and Youth Services Review, 35, 1101-1109. doi:10.1016/j.childyouth.2013.04.025

Leslie L.A., Smith, J.R., \& Hrapczynski, K.M. (2013). Racial socialization in transracial adoptive families: Does it help adolescents deal with discrimination stress? Family Relations, 62, 72 - 81. doi:10.1111/j.1741-3729.2012.00744.x

Leve, L.D., \& Chamberlain, p. (2007). A randomized evaluation of Multidimensional Treatment Foster Care: Effects on school attendance and homework completion in juvenile justice girls. Research on Social Work Practice, 17, 657-663. doi:10.1177/1049731506293971

Leve, L.D., Harold, G.T., Chamberlain, P., Landsverk, J.A., Fisher, P.A., \& Vostanis, P. (2012). Practitioner review: Children in foster care - vulnerabilities and evidencebased interventions that promote resilience processes. Journal of Child Psychology and Psychiatry, 53, 1197-1211. doi:10.1111/j.1469-7610.2012.02594.x

Lewis, E.E., Dozier, M., Ackerman, J., \& Sepulveda-Kozakowski, S. (2007). The effect of placement instability on adopted children's inhibitory control abilities and oppositional behavior. Developmental Psychology, 43, 1415-1427. doi:10.1037/00121649.43.6.1415

Lewis-Morrarty, E., Dozier, M., Bernard, K., Terracciano, S.M., \& Moore, S.V. (2012). Cognitive flexibility and theory of mind outcomes among foster children: Preschool follow-up results of a randomized clinical trial. Journal of Adolescent Health, 51, 517 - 522. doi:10.1016/j.jadohealth.2012.05.005 
Lichtenstein, J. \& Cassidy, J. (1991). The Inventory of Adult Attachment: Validation of a new measure. Paper presented at the Biennial Meeting of the Society for Research in Child Development, Seattle.

Linares, L. O., Montalto, D., Li, M., \& Oza, V.S. (2006). A promising parenting intervention in foster care. Journal of Consulting and Clinical Psychology, 74, 32-41. doi:10.1037/0022-006x.74.1.32

Lind, T., Bernard, K., Ross, E., \& Dozier, M. (2014). Intervention effects on negative affect of CPS-referred children: Results of a randomized clinical trial. Child Abuse and Neglect, 38, 1459-1467. doi:10.1016/j.chiabu.2014.04.004

Lindhiem, O., \& Dozier, M. (2007). Caregiver commitment to foster children: The role of child behaviour. Child Abuse and Neglect, 31, 361-374.

doi:10.1016/j.chiabu.2006.12.003

Litrownik, A.J., Lau, A., English, D.J., Briggs E., Newton, R.R., Romney S., \& Dubowitz, H. (2005). Measuring the severity of child maltreatment. Child Abuse and Neglect, 29, 553 - 573. doi:10.1016/j.chiabu.2003.08.010

Liu, J., \& Smith, B.D. (2011). Transferring training to child welfare practice: Individual and collective efforts. Children and Youth Services Review, 33, 149-156. doi:10.1016/j.childyouth.2010.08.027

Lok, S.M., \& McMahon, C.A. (2006). Mothers' thoughts about their children: Links between mind-mindedness and emotional availability. British Journal of Developmental Psychology, 24, 477-488. doi:10.1348/026151005x49854

Lovibond P., \& Lovibond S. (1995). The structure of negative emotional states: Comparison of the Depression Anxiety Stress Scales (DASS) with the Beck Depression and Anxiety Inventories. Behaviour, Research and Therapy, 33, 335-343. http://dx.doi:10.1016/0005-7967(94)00075-u

Lovibond, S.H., \& Lovibond, P.F. (1996). Manual for the depression, anxiety, and stress scales (2nd. ed.). Sydney: Psychological Foundation of Australia. 
Luke, N., \& Banerjee, R. (2013). Differentiated associations between childhood maltreatment experiences and social understanding: A meta-analysis and systematic review. Developmental Review, 33, 1-28. doi:10.1016/j.dr.2012.10.001

Lundy, B.L. (2003). Father- and mother-infant face-to-face interactions: Differences in mind-related comments and infant attachment? Infant Behavior and Development, 26, 200-212. doi:10.1016/s0163-6383(03)00017-1

Lynch, M., \& Cicchetti, D. (1997). Children's relationships with adults and peers: An examination of elementary and junior high school students. Journal of School Psychology, 35, 81-99. doi:10.1016/s0022-4405(96)00031-3

Lynch, F.L., Dickerson, J.F., Saldana, L., \& Fisher, P.A. (2014). Incremental net benefit of early intervention for preschool-aged children with emotional and behavioral problems in foster care. Children and Youth Services Review, 36, 213-219. doi:10.1016/j.childyouth.2013.11.025

Lyons, J.S., \& Rogers, L. (2004). The U.S. child welfare system: A de facto public behavioral health care system. Journal of the American Academy of Child and Adolescent Psychiatry, 43, 971-973. doi:10.1097/01.chi.0000127575.23736.90

Ma, H.-H. (2006). An alternative method for quantitative synthesis of single-subject researches: Percentage of data points exceeding the median. Behavior Modification, 30, 598-617. doi:10.1177/0145445504272974

Ma, H. H. (2009). The effectiveness of interventions on the behavior of individuals with autism: A meta-analysis using percentage of data points exceeding the median of baseline phase (PEM). Behavior Modification, 33, 339-359.

doi:10.1177/0145445509333173

Maaskant, A.M., van Rooij, F.B., \& Hermanns, J.M.A. (2014). Mental health and associated risk factors of Dutch school aged foster children placed in long-term foster care. Children and Youth Services Review, 44, 207-216. doi:10.1016/j.childyouth.2014.06.011 
Macdonald, G., \& Turner, W. (2005). An experiment in helping foster-carers manage challenging behaviour. British Journal of Social Work, 35, 1265-1282. doi:10.1093/bjsw/bch204

Macdonald, G.M., \& Turner, W. (2008). Treatment Foster Care for improving outcomes in children and young people. Cochrane Database of Systematic Reviews, 1, CD005649. doi:10.1002/14651858.CD005649.pub2

McCrory, E., De Brito, S.A., \& Viding, E. (2010). Research Review: The neurobiology and genetics of maltreatment and adversity. Journal of Child Psychology and Psychiatry, 51, 1079-1095. doi:10.1111/j.1469-7610.2010.02271.x

McLaughlin, K.A., Zeanah, C.H., Fox, N.A., \& Nelson, C.A. (2011). Attachment security as a mechanism linking foster care placement to improved mental health outcomes in previously institutionalized children. Journal of Child Psychology and Psychiatry and Allied Disciplines, 53, 46-55. doi:10.1111/j.1469-7610.2011.02437.x

McLean, S., Kettler, L., Delfabbro, P., \& Riggs, D. (2012). Frameworks for understanding challenging behaviour in out-of-home care. Clinical Psychologist, 16, 72-81. doi:10.1111/j.1742-9552.2011.00037.x

McLeod, G.F., Fergusson, D.M., \& Horwood, L.J. (2014). Childhood physical punishment or maltreatment and partnership outcomes at age 30. American Journal of Orthopsychiatry, 84, 307-315. doi:10.1037/h0099807

McMahon, C.A., \& Meins, E. (2012). Mind-mindedness, parenting stress, and emotional availability in mothers of preschoolers. Early Childhood Research Quarterly, 27, 245252. doi:10.1016/j.ecresq.2011.08.002

McMillen, J.C., Zima, B.T., Scott, L.D., Auslander, W.F., Munson, M.R., Ollie, M.T., \& Spitznagel, E.L. (2005). Prevalence of psychiatric disorders among older youths in the foster care system. Journal of the Academy of Child and Adolescent Psychiatry, 44, 8895. doi:10.1097/01.chi.0000145806.24274.d2

Main, M., Hesse, E., \& Kaplan, N. (2005). Predictability of attachment behaviour and representational processes at 1, 6, and 19 years of age: The Berkeley longitudinal study. 
In K.E. Grossmann, K. Grossmann, \& E. Waters, (Eds.). Attachment from infancy to adulthood: The major longitudinal studies (pp. 245-304). New York, NY: Guilford.

Mash, E.J., \& Wolfe, D.A. (2013). Abnormal Child Psychology ( $6^{\text {th }}$ ed.). Boston MA: Cengage Learning.

Meins, E. (1997). Security of attachment and the social development of cognition. Hove, U.K.: Psychology Press. doi:10.4324/9780203775912

Meins E. (1998).The effects of security of attachment and maternal attribution of meaning on children's linguistic acquisitional style. Infant Behavior and Development, 21, 237252. doi:10.1016/s0163-6383(98)90004-2

Meins, E. (2013). Sensitive attunement to infants' internal states: operationalizing the construct of mind-mindedness. Attachment and Human Development, 15, 524-544. doi:10.1080/14616734.2013.830388

Meins, E., Centifani, L.C.M., Fernyhough, C., \& Fishburn, S. (2013). Maternal mindmindedness and children's behavioral difficulties: Mitigating the impact of low socioeconomic status. Journal of Abnormal Child Psychology, 41, 543-553. doi:10.1007/s10802-012-9699-3

Meins, E., \& Fernyhough, C., (1999). Linguistic acquisitional style and mentalising development: The role of maternal mind-mindedness. Cognitive Development, 14, 363380. doi:10.1016/s0885-2014(99)00010-6

Meins, E, \& Fernyhough, C., (2010). Mind-mindedness coding manual, Version 2.0. Unpublished manuscript. Durham University, Durham, UK.

Meins, E., Fernyhough, C., Arnott, B., Leekam, S.R., \& De Rosnay, M. (2013). Mindmindedness and theory of mind: Mediating roles of language and perspectival symbolic play. Child Development, 84, 1777-1790. doi:10.1111/cdev.12061

Meins, E., Fernyhough, C., Fradley, E., \& Tuckey, M. (2001). Rethinking maternal sensitivity: Mothers' comments on infants' mental processes predict security of attachment at 12 months. Journal of Child Psychology and Psychiatry, 42, 637-648. doi:10.1111/1469-7610.00759 
Meins, E., Fernyhough, C., \& Harris-Waller., J. (2014). Is mind-mindedness trait-like or a quality of close relationships? Evidence from descriptions of significant others, famous people, and works of art. Cognition, 130, 417-427.

doi:10.1016/j.cognition.2013.11.009

Meins, E., Fernyhough, C., Russell, J., \& Clark-Carter, D., (1998). Security of attachment as a predictor of symbolic and mentalising abilities: A longitudinal study. Social Development, 7, 1-24. doi:10.1111/1467-9507.00047

Meins, E., Fernyhough, C., Wainwright, R., Clark-Carter, D., Das Gupta, M., Fradley, E., \& Tuckey, M. (2003). Pathways to understanding mind: Construct validity and predictive validity of maternal mind-mindedness. Child Development, 74, 1194-1211. doi:10.1111/1467-8624.00601

Mellor, D. (2004). Furthering the use of the Strengths and Difficulties Questionnaire: Reliability with younger child respondents. Psychological Assessment, 16, $396-401$. doi:10.1037/1040-3590.16.4.396

Mellor, D. (2005) Normative data for the Strengths and Difficulties Questionnaire in Australia. Australian Psychologist, 40, 215-222. doi:10.1080/00050060500243475

Meltzer, H., Gatward, R., Corbin, T., Goodman, R., \& Ford, T. (2003). The Mental Health of Young People Looked After by Local Authorities in England. London: Office for National Statistics.

Milan, S. E., \& Pinderhughes, E.E. (2000). Factors influencing maltreated children's early adjustment in foster care. Development and Psychopathology, 12, 63-81. doi:10.1017/s0954579400001048

Miller, K.M., Cahn, K., Anderson-Nathe, B., Cause, A.G., \& Bender, R. (2013). Individual and systemic/structural bias in child welfare decision making: Implications for children and families of color. Children and Youth Services Review, 35, 1634-1642. doi:10.1016/j.childyouth.2013.07.002

Miller, S.D., Duncan, B.L., Brown, J., Sparks, J.A. \& Claud, D.A. (2003). The Outcome Rating Scale: A preliminary study of the reliability, validity, and feasibility of a brief visual analog measure. Journal of Brief Therapy, 2, 91- 100. 
Milot, T., Ethier, L.S., St-Laurent, D., \& Provost, M.A. (2010). The role of trauma symptoms in the development of behavioral problems in maltreated preschoolers. Child Abuse and Neglect, 34, 225-234. doi:10.1016/j.chiabu.2009.07.006

Ministry of Social Development, Centre for Social Research and Evaluation (2011). Flow rates from Child, Youth and Family to Corrections - January 2011. Retrieved from: http://www.msd.govt.nz/documents/about-msd-and-our-work/flow rates from Child Youth and Family to Corrections.doc.

Ministry of Social Development, Centre for Social Research and Evaluation (2012). Home for life evaluation findings. Retrieved from:https://www.msd.govt.nz/about-msd-andour-work/publications-resources/evaluation/home-for-life/index.html

Minnis, H., Everett, K., Pelosi, A.J., Dunn, J., \& Knapp, M. (2006). Children in foster care: Mental health, service use and costs. European Child and Adolescent Psychiatry, 15, 63-70. doi:10.1007/s00787-006-0452-8

Minnis, H., Pelosi, A.J., Knapp, M., \& Dunn, J. (2001). Mental health and foster carer training. Archives of Disease in Childhood, 84, 302-306. doi:10.1136/adc.84.4.302

Murray, L., Tarren-Sweeney, M., \& France, K. (2011). Foster carer perceptions of support and training in the context of high burden of care. Child and Family Social Work, 16, 149-158. doi:10.1111/j.1365-2206.2010.00722.x

New Zealand Government (2012). The White paper for Vulnerable Children, Volume II. Retrieved from: http://www.msd.govt.nz/documents/about-msd-and-our-work/workprogrammes/policy-development/white-paper-vulnerable-children/whitepaper-volumeii-web.pdf

Newton, R.R., Litrownik, A.J., \& Landsverk, J.A. (2000). Children and youth in foster care: Disentangling the relationship between problem behaviors and number of placements. Child Abuse and Neglect, 24, 1363-1374. doi:10.1016/s0145-2134(00)00189-7

NICHD Early Child Care Research Network (2004). Affect dysregulation in the motherchild relationship in the toddler years: Antecedents and consequences. Development and Psychopathology, 16, 43-68. doi:10.1017/s0954579404044402 
Nilsen, W. (2007). Fostering futures: A preventive intervention program for school-age children in foster care. Clinical Child Psychiatry, 12, 45-62. doi:10.4135/9781452230689.n9

Nutt, L. (2006). The lives of foster carers: Private sacrifices, public restrictions. Abingdon Oxon: Routledge.

Ohan, J.L., Leung, D.W., \& Johnston, C. (2000). The Parenting Sense of Competency Scale: Evidence of a stable factor structure and validity. Canadian Journal of Behavioural Science, 32, 251-261. .doi:10.1037/h0087122

Oosterman, M., Schuengel, C., Wim Slot, N., Bullens, R.A.R., \& Doreleijers, T.A.H. (2007). Disruptions in foster care: A review and meta-analysis. Children and Youth Services Review, 29, 53-76. doi:10.1016/j.childyouth.2006.07.003

Oppenheim, D., \& Koren-Karie, N. (2013). The insightfulness assessment: Measuring the internal processes underlying maternal sensitivity. Attachment and Human Development, 15, 545-561. doi.org/10.1080/14616734.2013.820901

Oppenheim, D., Koren-Karie, N., \& Sagi, A. (2001). Mothers empathic understanding of their preschoolers' internal experience: Relations with early attachment. International Journal of Behavioral Development, 25, 16-26. doi:10.1080/01650250042000096

Organisation for Economic Co-operation and Development (2009). Doing Better For Children. Retrieved from: http://www.oecd.org/social/family/ doingbetterforchildren.htm. doi:10.1787/9789264059344-en

Organisation for Economic Cooperation and Development (2013). Family Violence Report. SF3.4 Family Violence. Retrieved from: www.oecd.org/social/family/database.

Osborn, A.L., Delfabbro, P., \& Barber, J.G. (2008). The psychosocial functioning and family background of children experiencing significant placement instability in Australian outof-home care. Children and Youth Services Review, 30, 847 - 860. doi:10.1016/j.childyouth.2007.12.012 
Oswald, S.H., Heil, K., \& Goldbeck, L. (2010). History of maltreatment and mental health problems in foster children: A review of the literature. Journal of Pediatric Psychology, 35, 462 - 472. doi:10.1093/jpepsy/jsp114

Park, J.M., Solomon, P., \& Mandell, D.S. (2007). Foster care placement among children who received inpatient psychiatric care. Children and Youth Services Review, 29, 933-940. doi:10.1016/j.childyouth.2006.12.010

Parker, R.I., \& Hagan-Burke, S. ( 2007). Median-based overlap analysis for single case data: A second study. Behavior Modification, 31, 919-936. doi:10.1177/0145445507303452

Parker, R.I., Vannest, K.J., \& Davis, J.L. (2011). Effect size in single-case research: A review of nine nonoverlap techniques. Behavior Modification, 35, 303-322. doi:10.1177/0145445511399147

Patterson, G. (2004). Evaluating the effects of child abuse training on the attitudes, knowledge and skills of police recruits. Research on Social Work Practice, 14, 273 280. doi: $10.1177 / 1049731503262390$

Pears, K.C., Bruce, J., Fisher, P.A., \& Kim, H.K. (2010). Indiscriminate friendliness in maltreated foster children. Child Maltreatment, 15, 64 - 75. doi: $10.1177 / 1077559509337891$

Pears, K., \& Fisher, P.A. (2005). Developmental, cognitive, and neuropsychological functioning in preschool-aged foster children: Associations with prior maltreatment and placement history. Journal of Developmental and Behavioral Pediatrics, 26, 112-122. doi:10.1097/00004703-200504000-00006

Pecora, P. J., Kessler, R.C., Williams, J., O’Brien, K., Downs, A.C., English D.,...Holmes, K. (2005). Improving family foster care: Findings from the Northwest foster care alumni study. Casey Family Programs, Seattle, WA. Retrieved from: http://www.casey.org/Resources/Publications/CFOMH.htm

Pianta, R. (1994). The Child-Parent Relationship Scale. Charlottesville, VA: University of Virginia. 
Pithouse, A., Hill-Tout, J., \& Lowe, K. (2002). Training foster carers in challenging behaviour: A case study in disappointment? Child and Family Social Work, 7, 203214.

Pollock, K. (2013). 'Children's homes and fostering', Te Ara - the Encyclopedia of New Zealand, updated 20-Jun-13. URL: http://www.TeAra.govt.nz/en/childrens-homesand-fostering/sources

Ponciano, L. (2010). Attachment in foster care: The role of maternal sensitivity, adoption, and foster mother experience. Child and Adolescent Social Work Journal, 27, 97-114. doi:10.1007/s10560-010-0192-y

Price, J.M., Chamberlain, P., Landswerk, J., Reid, J., Leve L.D., \& Laurent, H. (2008). Effects of a foster parent training intervention on placement changes of children in foster care. Child Maltreatment, 13, 64-75. doi:10.1177/1077559507310612

Price, J.M., Roesch, S.C., \& Walsh, N.E. (2012). Effectiveness of the KEEP foster parent intervention during an implementation trial. Children and Youth Services Review, 34, 2487-2494. doi:10.1016/j.childyouth.2012.09.010

Pritchett, R., Gillberg, C., \& Minnis, H. (2013). What do child characteristics contribute to outcomes from care: A PRISMA review. Children and Youth Services Review, 35, 1333-1341. doi:10.1016/j.childyouth.2013.05.001

Proctor, L.J., Van Dusen Randazzo, K., Litrownik, A.J., Newton, R.R., Davis, I.P., \& Villodas (2011). Factors associated with caregiver stability in permanent placements: A classification tree approach. Child Abuse and Neglect, 35, pp. 425-436. doi:10.1016/j.chiabu.2011.02.002

Puddy, R.W., \& Jackson, Y. (2003). The development of parenting skills in foster parent training. Children and Youth Services Review, 25, 987-1013. doi:10.1016/s01907409(03)00106-3

Rankin (2011). Meeting the needs of New Zealand children and young people who have been abused and neglected. Best Practice Journal, 37, 4-9. http://www.bpac.org.nz/BPJ/2011/august/upfront.aspx 
Raviv, T., Taussig, H.N., Culhane, S.E., \& Garrido, E.F. (2010). Cumulative risk exposure and mental health symptoms among maltreated youth placed in out-of-home care. Child Abuse and Neglect, 34, 742-751. doi:10.1016/j.chiabu.2010.02.011

Rees, P. (2013). The mental health, emotional literacy, cognitive ability, literacy attainment and 'resilience' of 'looked after children': A multidimensional, multiple-rater population based study. British Journal of Clinical Psychology, 52, 183-198. doi:10.1111/bjc. 12008

Rhoades, K. A., Chamberlain, P., Roberts, R., \& Leve, L. D. (2013). MTFC for high risk adolescent girls: A comparison of outcomes in England and the US. Journal of Child and Adolescent Substance Abuse, 22, 435-449. doi:10.1080/1067828x.2013.788887

Rhoades, K.A., Leve, L.D., Harold, G.T., Kim, H.K., \& Chamberlain, P. (2014). Drug use trajectories after a randomized controlled trial of MTFC: Associations with partner drug use. Journal of Research on Adolescence, 24, 40-54. doi:10.1111/jora.12077

Ronk, F.R., Korman, J.R., Hooke, G.R., \& Page, A.C. (2013). Assessing clinical significance of treatment outcomes using the DASS-21. Psychological Assessment, 25, 1103-1110. doi: $10.1037 / \mathrm{a} 0033100$

Rork, K. E., \& McNeil, C. B. (2011). Evaluation of foster parent training programs: A critical review. Child and Family Behavior Therapy, 33, 139-170. doi:10.1080/07317107.2011.571142

Rosenblum, K.L., McDonough, S.C., Sameroff A.J., \& Muzik, M. (2008). Reflection in thought and action: Maternal parenting reflectivity predicts mind-minded comments and interactive behavior. Infant Mental Health Journal, 29, 362-376. doi:10.1002/imhj.20184

Rubin, D.M., Alessandrini, E.E., Feudtner, C., Mandell, D.S., Localio, A.R., \& Hadley, T. (2004). Placement stability and mental health costs for children in foster care. Pediatrics, 113, 1336-1341. doi:10.1542/peds.113.5.1336

Rubin, D.M., O'Reilly, A.L.R., Luan, X., \& Localio, A.R. (2007). The impact of placement stability on behavioral well-being for children in foster care. Pediatrics, 119, 336-344. doi:10.1542/peds.2006-1995 
Rushton, A. (2004). A Scoping and Scanning Review of Research on the Adoption of Children Placed from Public Care. Clinical Child Psychology and Psychiatry, 9, 89106. doi:10.1177/1359104504039768

Rutter, M. (1979). Protective factors in children's responses to stress and disadvantage. In M.W. Kent \& J.E. Rolf (Eds.), Primary prevention of psychopathology, Vol.3: Social competence in children (pp. 49-74). Hanover, NH: University of New England Press

Ryan, J.P., \& Testa, M.F. (2005). Child maltreatment and juvenile delinquency: Investigating the role of placement and placement instability. Children and Youth Services Review, 27, 227-249. doi:10.1016/j.childyouth.2004.05.007

Sameroff, A. (2009). The transactional model. In A. Sameroff (Ed.) The transactional model of development: How children and contexts shape each other (pp. 3-21). Washington DC: American Psychological Association.

Sanders, M.R., Kirby, J.N., Tellegen, C.L., \& Day, J.J. (2014). The Triple P-Positive Parenting Program: A systematic review and meta-analysis of a multi-level system of parenting support. Clinical Psychology Review, 34, 337-357. doi:10.1016/j.cpr.2014.04.003

Sanders, M.R., Pidgeon, A,M., Gravestock, F., Connors, M.D., Brown, S., \& Young, R.W. (2004). Does parental attributional retraining and anger management enhance the effects of the Triple P-Positive parenting Program with parents at risk of child maltreatment? Behaviour Therapy, 35, 513-535. doi:10.1016/s0005-7894(04)80030-3

Schofield G., \& Beek, M. (2005a). Providing a secure base: Parenting children in long-term foster family care. Attachment and Human Development, 7, 3-26. doi:10.1080/14616730500049019

Schofield G., \& Beek, M. (2005b). Risk and resilience in long-term foster-care. British Journal of Social Work, 35, 1283-1301. doi:10.1093/bjsw/bch213

Schofield, G., Beek, M., \& Ward, E. (2012). Part of the family: Planning for permanence in long-term family foster care. Children and Youth Services Review, 34, 244-253. doi:10.1016/j.childyouth.2011.10.020 
Schroder, R., Sellman, D., Frampton, C., \& Deering, D. (2008). Profile of young people attending alcohol and other drug treatment services in Aotearoa, New Zealand: Clinical file search. Australian and New Zealand Journal of Psychiatry, 42, 963 - 968. doi:10.1080/00048670802415368

Scott, K.M., Smith, D.R., \& Ellis, P.M. (2010). Prospectively ascertained child maltreatment and its association with DSM-IV mental disorders in young adults. Archives of General Psychiatry, 67, 712-719. doi:10.1001/archgenpsychiatry.2010.71

Scott, S., Spender, Q., Doolan, M., Jacobs, B., \& Aspland, H. (2001). Multicentre controlled trial of parenting groups for childhood antisocial behaviour in clinical practice. British Medical Journal, 323, 194-197. doi:10.1186/isrctn94713762

Searle, A.K., Miller-Lewis, L.R., Sawyer, M.G., \& Baghurst, P.A. (2013). Predictors of Children's Kindergarten Classroom Engagement: Preschool Adult-Child Relationships, Self-Concept, and Hyperactivity/Inattention. Early Education and Development, 24, 1112 - 1136. doi:10.1080/10409289.2013.764223

Sharp, C., \& Fonagy, P. (2008). The parent's capacity to treat the child as a psychological agent: Constructs, measures and implications for developmental psychopathology. Social Development, 17, 738-754. doi:10.1111/j.1467-9507.2007.00457.x

Shipman, K.L., Schneider, R., Fitzgerald, M.M., Sims, C., Swisher, L., \& Edwards, A. (2007). Maternal emotion socialization in maltreating and non-maltreating families: Implications for children's emotion regulation. Social Development, 16, 268285. doi:10.1111/j.1467-9507.2007.00384.x

Simkiss, D.E., Stallard, N., \& Thorogood, M. (2013). A systematic literature review of the risk factors associated with children entering public care. Child: Care, Health and Development, 39, 628-642. doi:10.1111/cch.12010

Simmel, C., Brooks, D., Barth, R.P., \& Hinshaw, S.P. (2001). Externalizing symptomatology among adoptive youth: Prevalence and preadoption risk factors. Journal of Abnormal Child Psychology, 29, 57-69. 
Smith, D.A.R., \& Beautrais, A. (1999). Identifying young people at risk of suicide. Social Work Now, December, 1999. Retrieved from: http://www.cyf.govt.nz/documents/ about-us/publications/social-work-now/social-work-now-14-dec99.pdf

Smith, D.K., Chamberlain, P., \& Eddy, J.M. (2010). Preliminary support for multidimensional treatment foster care in reducing substance use in delinquent boys. Journal of Child and Adolescent Substance Abuse, 19, 343-358. doi:10.1080/1067828x.2010.511986

Sobell, M.B., Sobell, L.C., \& Gavin, D.R. (1995). Portraying alcohol treatment outcomes: Different yardsticks of success. Behavior Therapy, 26, 643 - 669. doi:10.1016/s00057894(05)80037-1

Sprang, G. (2009). The efficacy of a relational treatment for maltreated children and their families. Child and Adolescent Mental Health, 14, 81-88. doi:10.1037/e517292011335

Sroufe, A. (2000). Early relationships and the development of children. Infant Mental Health Journal, 21, 67 - 74.

Stahmer, A.C., Leslie, L.K., Hurlburt, M., Barth, R.P., Webb, M.B., Landsverk, J., \& Zhang, J. (2005). Developmental and behavioural needs and service use for young children in child welfare. Pediatrics, 116, 891-900. doi:10.1542/peds.2004-2135

Stams, G-J.J.M., Juffer, F., van IJzendoorn, M.H., \& Hoksbergen, R.A.C. (2001). Attachment-based intervention in adoptive families in infancy and children's development at age seven: Two follow-up studies. British Journal of Developmental Psychology, 19, 159-180. doi:10.1348/026151001166010

Steele, J.S., \& Buchi, K.F. (2008). Medical and mental health of children entering the Utah foster care system. Pediatrics, 122, e703-e709. doi:10.1542/peds.2008-0360

Steele, K., Hodges, J., Kaniuk, J., Hillman, S., \& Henderson, K. (2003). Attachment representations and adoption: Associations between maternal states of mind and emotion narratives in previously maltreated children. Journal of Child Psychotherapy, 29, 187-205. doi:10.1080/0075417031000138442 
Stovall, K.C., \& Dozier, M. (2000). The development of attachment in new relationships. Single subject analyses for 10 foster infants. Development and Psychopathology, 12, 133-156. doi:10.1017/s0954579400002029

Stovall-McClough, K.C., \& Dozier, M. (2004). Forming attachments in foster care: Infant attachment behaviours during the first 2 months of placement. Development and Psychopathology, 16, 253-271. doi:10.1017/s0954579404044505

Stronach, E.P., Toth, S.L., Rogosch, F., Oshri, A., Manly, J.T., \& Cicchetti, D. (2011). Child maltreatment, attachment security, and internal representations of mother and motherchild relationships. Child Maltreatment, 16, 137-145. doi:10.1177/1077559511398294

Stunkard, A.J., \& Penick, S.B. (1979). Behavior modification in the treatment of obesity. Archives of General Psychiatry, 36, 801-806.

Swanson,K., Beckwith, L., \& Howard, J. (2000). Intrusive caregiving and quality of attachment in prenatally drug-exposed toddlers and their primary caregivers. Attachment and Human Development, 2, 130-148. doi:10.1080/14616730050085527

Tarren-Sweeney, M. (2008a). Retrospective and concurrent predictors of the mental health of children in care. Children and Youth Services Review, 30, 1-25. doi:10.1016/j.childyouth.2007.05.014

Tarren-Sweeney, M., (2008b). The mental health of children in out-of-home care. Current Opinion in Psychiatry, 21, 345-349. doi:10.1097/yco.0b013e32830321fa

Tarren-Sweeney, M. (2013). An investigation of complex attachment- and trauma-related symptomatology among children in foster and kinship care. Child Psychiatry and Human Development, 44, 727-741. doi:10.1007/s10578-013-0366-x

Taussig, H.N., \& Culhane, S.E. (2010). Impact of a mentoring and skills group program on mental health outcomes for maltreated children in foster care. Archives of Pediatrics and Adolescent Medicine, 164, 739-746. doi:10.1001/archpediatrics.2010.124

Taussig, H.N., Harpin, S. B., \& Maguire, S.A., (2014). Suicidality among preadolescent maltreated children in foster care. Child Maltreatment, 19, 17-26. doi:10.1177/1077559514525503 
Thompson, R. (2000). The legacy of early attachments. Child Development, 71, 145-152. doi:10.1111/1467-8624.00128

Timmer, S.G., Urquizer, A.J., \& Zebell, N. (2006). Challenging foster caregiver-maltreated child relationships: The effectiveness of parent-child interaction therapy. Children and Youth Services Review, 28, 1-19. doi:10.1016/j.childyouth.2005.01.006

Toth, S.L., Gravener-Davis, J.A., Guild, D.J., \& Cicchetti, D. (2013). Relational interventions for child maltreatment: Past, present, and future perspectives. Development and Psychopathology, 25, 1601-1617. doi:10.1017/s0954579413000795

Toth, S.L., Maughan, A., Manly, J.T., Spagnola, M., \& Cicchetti, D. (2002). The relative efficacy of two interventions in altering maltreated preschool children's representational models: Implications for attachment theory. Development and Psychopathology, 14, 877-908. http://dx.doi:10.1017/s095457940200411x

Trapolini, T., Ungerer, J.A., \& McMahon, C.A. (2008). Maternal depression: relations with maternal caregiving representations and emotional availability during the preschool years. Attachment and Human Development, 10, 73-90.

doi:10.1080/14616730801900712

Turner, W., \& Macdonald, G. (2011). Treatment foster care for improving outcomes in children and young people: A systematic review. Research on Social Work Practice, 21, 501-527. doi:10.1177/1049731511400434

Turner W., Macdonald G.M., \& Dennis J.A. (2007). Behavioural and cognitive behavioural training interventions for assisting foster carers in the management of difficult behaviour. Cochrane Database of Systematic Reviews. Issue 1. Art. No.: CD003760. DOI: 10.1002/14651858.CD003760.pub3.

UNICEF (2003). A league table of child maltreatment deaths in rich nations, Innocenti Report Card No.5, September. UNICEF Innocenti Research Centre, Florence. Retrieved from: http://www.unicef-irc.org/publications/pdf/repcard5e.pdf

Unrau, Y.A., Seita, J.R., \& Putney, K.S. (2008). Former foster youth remember multiple placement moves: A journey of loss and hope. Children and Youth Services Review, 30, 1256-1266. doi:10.1016/j.childyouth.2008.03.010 
Unrau, Y. A., Font, S.A., \& Murphy, S.R. (2011). Placement moves of foster children as perceived by foster parents residing in urban and semi-urban communities. Journal of Public Child Welfare, 5, 390-407. doi:10.1080/15548732.2011.599757

U.S. Department of Health and Human Services USA (2013). Data Brief 2013-1. ACYF Office of Data, Analysis, Research, and Evaluation. Retrieved from: http://www.acf. hhs.gov/sites/default/files/cb/data_brief_foster_care_trends1.pdf

van den Dries, L., Juffer, F., van Ijzendoorn, M., \& Bakermans-Kranenburg (2009). Fostering security? A meta-analysis of attachment in adopted children. Children and Youth Services Review, 31, 410-421. doi:10.1016/j.childyouth.2008.09.008

Van IJzendoorn, M.H. (1995). Adult attachment representations, parental responsiveness, and infant attachment: A meta-analysis on the predictive validity of the adult attachment interview. Psychological Bulletin, 117, 387-403. doi:10.1037//00332909.117.3.387

van IJzendoorn, M.H., Schuengel, C. \& Bakermans-Kranenburg, M.J. (1999). Disorganized attachment in early childhood: Meta-analyses of precursors, concomitants and sequelae. Development and Psychopathology, 11, 225-249. doi:10.1017/s0954579499002035

Walker, T.M., Wheatcroft, R., \& Camic, P.M. (2012). Mind-mindedness in parents of preschoolers: A comparison between clinical and community samples. Clinical Child Psychology and Psychiatry, 17, 318-335. doi:10.1177/1359104511409142

Warman, A., Pallett, C., \& Scott, S. (2006). Learning from each other: Process and outcomes in the Fostering Changes training programme. Adoption and Fostering, 30, 17-28. doi:10.1177/030857590603000304

Webster-Stratton, C. \& Reid, M. J. (2010): The incredible years parent, teachers, and children training series. A multifaceted treatment approach for young children with conduct disorders. In J. R. Weisz \& A. E. Kazdin (Eds.), Evidence-based psychotherapies for children and adolescents (2nd ed., pp. 194-210). New York NY: Guilford Press. 
Weiner, D. A., Schneider A., \& Lyons, J. (2009). Evidence-based treatments for trauma among culturally diverse foster care youth: treatment retention and outcomes. Child and Youth Services Review, 31, 1199 - 1205.

Weir, K.N., Fife, S.T., Whiting, J.B., \& Blazewick, A. (2008). Clinical training of MFTs for adoption, foster care and child development settings: A comparative survey of CACREP, COAMFTE and CSWE accredited programs. Journal of Family Psychotherapy, 19, 277- 290. doi:10.1080/08975350802269517

Wells, P.M. (2004). “Someone to walk with me”; Supporting caregivers who look after children in with mental health problems in statutory care (Unpublished doctoral dissertation). Massey University, Wellington, NZ.

Westermark, P.K., Hansson, K., \& Olsson, M. (2011). Multidimensional treatment foster care (MTFC): Results from an independent replication. Journal of Family Therapy, 33, 20-41. doi:10.1111/j.1467-6427.2010.00515.x

Westermark, P.K., Hansson, K.,\& Vinnerljung, B. (2007). Foster parents in Multidimensional Treatment Foster Care: How do they deal with implementing standardized treatment components? Children and Youth Services Review, 29, 442459. doi:10.1016/j.childyouth.2006.10.003

White, C.R., Havalchak, A., Jackson, L.J., O’Brien, K., \& Pecora, P.J. (2007). Mental health, ethnicity, sexuality and spirituality among youth in foster care: Findings from the Casey Field Office study. Casey Family Programs, Seattle, WA. Retrieved from: http://www.casey.org/Resources/Publications/CFOMH.htm

White, C.R., O’Brien, K., Pecora, P.J., English, D., Williams, J.R., \& Phillips, C.M. (2009). Depression among alumni of foster care: Decreasing rates through improvement of experiences in care. Journal of Emotional and Behavioral Disorders, 17, 38-48. doi:10.1177/1063426608320356

Wilson, K. (2006). Can foster carers help children resolve their emotional and behavioural difficulties? Clinical Child Psychology and Psychiatry, 11, 495-511. doi:10.1177/1359104506067873 
Wotherspoon, E., O’Neill-Laberge, M., \& Pirie, J. (2008). Meeting the emotional needs of infants and toddlers in foster care: The collaborative mental health care experience. Infant Mental Health Journal,29, 377-397. doi:10.1002/imhj.20185

Yates, A.M., Lekies, K.S., Stockdale, D.F., \& Crase, S.J. (1997). Motivations for foster parenting inventory. Ames, IA: Iowa State University Research Foundation.

Zanov, M.V., \& Davison, G.C. (2010). A conceptual and empirical review of 25 years of cognitive assessment using the articulated thoughts in simulated situations (ATSS) think-aloud paradigm. Cognitive Therapy and Research, 34, 282-291. doi:10.1007/s10608-009-9271-9

Zeanah, C.H., Benoit, D., \& Barton, M.L. (1986). Working Model of the Child Interview. Brown University Program in Medicine. Providence, NY.

Zeanah, C.H., Larrieu, J.A., Heller, S.S., Valliere, J., Hinshaw-Fuselier, S., Aoki, Y., \& Drilling, M. (2001). Evaluation of a Preventive Intervention for Maltreated Infants and Toddlers in Foster Care. Journal of the American Academy of Child and Adolescent Psychiatry, 40, 214-221. doi:10.1097/00004583-200102000-00016 
